Neurocritical Care Society Scientific Abstracts from

the 19th Annual Meeting

October 26-29, 2021 


\title{
Oral Abstract 1
}

\section{Blood Clot Burden can Differentiate Perimesencephalic Subarachnoid Hemorrhage from Aneurysmal Etiology}

\author{
Daniel Mandel, Scott Moody, Kelly Pan, Bradford B. Thompson, Linda C. Wendell, Michael E. Reznik, \\ Karen L. Furie, Ali Mahta
}

Brown University, Providence, RI, United States

\section{Background \& Purpose}

Perimesencephalic subarachnoid hemorrhage (PMSAH) is a predominantly benign pattern of subarachnoid hemorrhage. However, in a minority of PMSAH, a posterior circulation aneurysm is causative. Out of concern for missing an aneurysm, many institutions pursue exhaustive imaging. We aimed to design a predictive model, based on radiographic features of admission non-contrast head computerized tomography (NCHCT), to differentiate PMSAH from aneurysmal causes.

\section{Methods}

We retrospectively reviewed consecutive patients admitted for suspected aneurysmal SAH (aSAH) to an academic center. Patients with a final diagnosis of PMSAH or posterior circulation aSAH were included. Using NCHCT, thickness (continuous variable) and location of blood in basal cisterns and Sylvian fissures (categorical variables) were compared between groups. Using the statistically significant features, we created a scoring system. Receiver operating characteristics (ROC) were used to measure accuracy of this model in predicting aneurysmal etiology.

\section{Results}

Of 420 SAH cases, we identified 56 with PMSAH and 48 with posterior circulation aSAH. Mean age was 54.7 years (SD 12.6) and 58 patients (54\%) were female. Blood thickness measurements in the crural and ambient cisterns, interhemispheric and Sylvian fissures, and degree of extension into the Sylvian fissure were all statistically significant ( $p=0.001,<0.001,<0.001,<0.001$, and $<0.001$, respectively). Using these significant figures, we developed a 10-point scoring model to predict aneurysmal causes with high accuracy (area under the curve [AUC] 0.98; 95\% Cl 0.96-1.00; odds ratio per point increase: $7.6 ; 95 \% \mathrm{Cl}$ 2.6-22).

\section{Conclusions}

If externally validated, our predictive model may assist clinicians in the risk-stratification of patients presenting with PMSAH. This model could minimize protracted admissions to intensive care units and reduce healthcare resource utilization and costs. 


\title{
Oral Abstract 2
}

\section{Leucine-rich Alpha-2-glycoprotein 1 is Systemic Biomarker of Early Brain Injury and Delayed Cerebral Ischemia after Subarachnoid Hemorrhage}

\author{
H. Alex Choi, Jude PJ Savarraj, Eunsu Park, Sarah Hinds, Atzhiry Paz, Aaron M. Gusdon, Hilda Ahnstedt, \\ Xuefang Ren, Louise McCullough, Devin W. McBride \\ UT Health Neuroscience, Houston, TX, United States
}

\section{Background \& Purpose}

Early brain injury $(\mathrm{EBI})$ and delayed cerebral ischemia $(\mathrm{DCl})$ are pathophysiological processes that lead to poor outcomes after subarachnoid hemorrhage (SAH). The key biological mechanisms are poorly understood. We performed untargeted proteomics, validation studies and confirmatory animal studies identifying Leucine-rich alpha-2-glycoprotien 1 (LRG1) as a biomarker for EBI and DCI.

\section{Methods}

In a prospective cohort study, plasma samples were collected within 48 hours after SAH. Untargeted proteomics using mass spectrometry was performed for biomarkers of $\mathrm{EBI}$ and $\mathrm{DCl}$ in two separate cohorts ( 27 subjects and 45 subjects). Confirmatory ELISAs for our candidate biomarker was performed in an independent validation cohort of 72 patients. Plasma and CSF levels of LRG1 were compared across EBI severity (SEBES $\geq 3 \vee S E B E S \leq 2$ ), and $D C I$. In a murine model of $S A H$, we measured LRG1 levels in plasma and brain tissue. We compared levels of LRG1 in SAH versus control sham animals. MannWhitney $\mathrm{U}$ test was used for statistical comparisons.

\section{Results}

Plasma LRG1 was the only marker which was associated with $\mathrm{EBI}$ and $\mathrm{DCl}$ in both filter cohorts (untargeted proteomics). In an independent validation cohort we found that levels of plasma LRG1 were associated with EBI $(99 \pm 5.1 \mathrm{pg} / \mathrm{ml}$ vs. $69 \pm 2.61 \mathrm{pg} / \mathrm{ml}, \mathrm{p}<0.05)$ and $\mathrm{DCl}(95 \pm 5.3 \mathrm{pg} / \mathrm{ml} \mathrm{vs.} 72 \pm 3.6 \mathrm{pg} / \mathrm{ml}$, $p<0.05)$. CSF levels of LRG1 were not associated with clinical variables. Similarly, in our experimental model of SAH, LRG1 was significantly elevated in plasma $(p<0.001)$ but not in brain tissue. Plasma LRG1 was associated with worse function as measured by the composite Neuroscore) $(r=-0.93, p=0.005$; Spearman coefficient).

\section{Conclusions}

Using an untargeted approach with confirmatory testing across three cohorts, we identified LRG1 as a biomarker for EBI and DCI. Experimental SAH caused elevated peripheral LRG1 levels without elevated brain levels, mirroring our clinical findings. Further studies to elucidate the role of LRG1 in the pathophysiology of SAH are needed. 


\title{
Oral Abstract 3
}

\section{Evaluation of the Interaction between Anti-Platelet Therapy and Intraoperative Opioids in Patients Undergoing Neuroendovascular Stenting}

\author{
Blake Robbins, Casey May, Devin Holden, Aaron Cook, the Neurocritical Care Pharmacy Section Research \\ Group
}

University of Kentucky Healthcare Department of Pharmacy Services, Lexington, KY, United States

\section{Background \& Purpose}

Dual antiplatelet therapy (DAPT) is commonly employed peri-procedurally for neuroendovascular stenting due to the significant risk of thromboembolic events associated with stenting. A growing body of evidence in cardiac literature suggests that opioids may inhibit and delay the absorption of oral antiplatelet agents, contributing to antiplatelet resistance. The objective of this study was to evaluate the influence of intraoperative opioids on clinical outcomes of bleeding and thrombosis in patients receiving DAPT who have undergone neuroendovascular stenting.

\section{Methods}

This was a multicenter, retrospective cohort study of patients who underwent neuroendovascular stenting and received DAPT therapy between $7 / 1 / 17$ and $10 / 31 / 20$. Subjects were allocated into groups based on procedural opioid exposure. The primary outcome was the effect of opioid administration on platelet reactivity with nonresponse defined as a VerifyNow PRU $>208$ or by platelet aggregation testing. Patients were included in the primary endpoint if they had antiplatelet testing performed during or within 48 hours of the procedure. Secondary outcomes included thrombosis or bleeding within 48 hours after the procedure and the use of rescue agents.

\section{Results}

In an interim analysis, 283 patients received opioids and 83 patients received no-opioids intraoperatively. Mean age was 62 years, with $71 \%$ of patients undergoing a planned procedure. Overall indications were $35 \%$ ICA stenting, 33\% unruptured aneurysm, 16\% thrombectomy, $8 \%$ ruptured aneurysm), $3 \%$ artery dissection, and $5 \%$ other indications. A total of 44 patients were included in the primary endpoint (36 in opioid group and 8 in non-opioid group). Of these, non-response was found in $19 \%$ of patients receiving opioids vs. $0 \%$ in patients not receiving opioids $(P=0.17)$.

\section{Conclusions}

Employing opioids intraoperatively for neuroendovascular procedures may lead to a greater number of antiplatelet non-responders based on antiplatelet function testing. Continued analysis on the full cohort and clinical outcomes is pending. 


\title{
Oral Abstract 4
}

\section{Predictive Value of Structural Connectome Changes in the Acute Phase of Aneurysmal Subarachnoid Hemorrhage: A Graph Analysis of Diffusion Tensor Imaging Data}

\author{
Sarah E. Nelson, Casey Weiner, Alexander Sigmon, Jun Hua, Haris I. Sair, Jose I. Suarez, Robert D. \\ Stevens
}

Icahn School of Medicine at Mount Sinai, New York, NY, United States

\section{Background \& Purpose}

We hypothesized that structural connectivity changes would occur in canonical brain networks of acute aSAH patients, and that these changes would be associated with functional outcome.

\section{Methods}

In a prospective cohort of patients admitted to a single institution for management of acute aSAH, patients underwent diffusion tensor imaging (DTI) as part of a multimodal MRI scan. A weighted undirected structural connectome was created of each patient's images using 176 regions of interest (ROIs) defined by the Johns Hopkins Eve atlas, and ROls were sorted into four networks: Default Mode Network, Executive Control Network, Salience Network, and Whole Brain. Resulting nodes and edges were characterized using graph theoretic features including Node Strength (NS), Betweenness Centrality (BC), Network Degree (ND), and Connectedness (C). Clinical (including demographics and World Federation of Neurologic Surgeons scale) and graph features were used separately and in combination to train Random Forest (RF) and Logistic Regression classifiers to predict two outcomes measured as the dichotomized modified Rankin Score (mRS) at discharge and at six months after discharge (favorable outcome mRS 0-2, unfavorable outcome mRS 3-6).

\section{Results}

A total of 56 aSAH patients underwent DTI a median (IQR) of 7 (IQR=8.5) days after admission. The best performing model (RF) combining clinical and DTI graph features had a mean Area Under the Receiver Operator Characteristic Curve (AUROC) of 0.88 and an Area Under the Precision Recall Curve (AUPRC) of 0.95 for prediction of discharge mRS. The combined model performed better than the clinical model alone (AUROC $0.81 \pm 0.01$, AUPRC $0.91 \pm 0.00$ ). The highest ranked graph features for prediction were NS, BC, and ND.

\section{Conclusions}

Results indicate that performance of clinical prognostic models is increased significantly by the inclusion of DTI-derived graph connectivity metrics. They point to the clinical impact of connectome reorganization early after aSAH. 


\title{
Oral Abstract 5
}

\section{Esmolol Reduces Anterior Circulation Cerebral Perfusion Compared to Nicardipine}

\author{
Michael Gezalian, Shouri Lahiri \\ Cedars-Sinai Medical Center, Department of Neurology/Neurocritical Care, Los Angeles, CA, United \\ States
}

\section{Background \& Purpose}

Nicardipine, a dihydropyridine calcium channel blocker, and esmolol, a beta-1 receptor antagonist, are guideline-endorsed anti-hypertensive infusions for acute brain injury conditions. In non-injured brains with intact cerebral autoregulation, we expect that modest reductions in systemic mean arterial blood pressure (MAP) with nicardipine or esmolol infusion will have no significant effect on cerebral perfusion.

\section{Methods}

Eleven spontaneously hypertensive rats (15-24 weeks old) were randomized to receive either esmolol $(n=5)$ or nicardipine $(n=6)$ infusion. Animals underwent femoral vein catheter and arterial line placement for medication administration and continuous MAP monitoring, respectively. A 9.4T preclinical-MRI scanner was used to quantify cerebral perfusion in the proximal anterior circulation using arterial spin labeling. Internal carotid artery and jugular venous flow was quantified using 2D-phase contrast MRI sequencing. In each rat, MRI was performed at baseline and after MAP reduction by $25 \%$ with either nicardipine or esmolol infusion.

\section{Results}

There were no significant differences in baseline MAP, age, or weight between the two groups, and no significant difference in MAP reduction between the two groups as well (-26.75\% for nicardipine and $27.59 \%$ for esmolol, $p=0.44)$. However, there was a significant reduction in cerebral perfusion with esmolol infusion compared to nicardipine $(p=0.01)$. Furthermore, there was a significant decrease in carotid artery flow $(p=0.03)$ and jugular flow $(p=0.01)$ with esmolol infusion, as well as a strong trend toward decreased anterior cerebral perfusion $(p=0.06)$. In contrast, there was no significant difference in cerebral perfusion $(p=0.46)$, carotid flow $(p=0.14)$, or jugular flow $(p=0.06)$ with nicardipine infusion.

\section{Conclusions}

Our data indicates that esmolol significantly reduces cerebral perfusion compared to nicardipine. These findings suggest that esmolol may impair cerebral autoregulation and inadvertently contribute to cerebral hypoperfusion. Future studies are needed to understand the specific mechanism by which esmolol impairs cerebral autoregulation and to correlate these hemodynamic changes to structural brain injury markers. 


\section{Oral Abstract 6}

\section{Sleep Disturbances and Quality of Life in Children after Critical Care Hospitalization}

\section{Amy Colagiovanni Morrison, Trevor A. Hall, Julie Randall, Grace Recht, Cydni N. Williams}

Oregon Health \& Science University: Pediatric Critical Care and Neurotrauma Recovery Program, Portland, OR, United States

\section{Background \& Purpose}

More than 60,000 children with acquired brain injury ( $\mathrm{ABI}$ ) require neurocritical care annually. Over $50 \%$ suffer sleep-wake disturbances (SWDs) in the months after hospital discharge. Given the importance of sleep to brain development and repair after injury, we hypothesized SWD would be associated with worse global health outcomes measured by health related quality of life (HRQOL).

\section{Methods}

Prospective observational study of $\mathrm{N}=151$ children with $\mathrm{ABI}$ aged 3-18 years evaluated 1-3 months after critical care hospitalization. SWDs were measured by the Sleep Disturbances Scale for Children (SDSC) and defined as any T-score $\geq 60$ signifying moderate or severe risk of clinical sleep disorders. Overall HRQOL was measured by the Pediatric Inventory for Quality of Life (PedsQL), which defines the Minimal Clinically Important Difference (MCID) as 4.5 points in the total score. Secondary outcomes included PedsQL scores for physical, emotional, social, and school health domains. SWD groups were compared with Mann Whitney tests. Multiple linear regression evaluated association between SWD and overall $\mathrm{HRQOL}$ controlling for patient and ABI characteristics. Significance defined as $p<.05$.

\section{Results}

SWDs were present in $66 \%$ ( $n=100)$. HRQOL total score was significantly lower in children with SWD ( median=70; IQR=54, 80) versus without SWD (median=85; IQR=67, 94; $p<.001$ ). Median scores in emotional, social, and school domains were significantly lower in the SWD group (all $p<.01$ ). When controlling for age, sex, critical care interventions, pre-admission comorbidities, and decline from preadmission Functional Status Scale score, presence of SWD significantly reduced HRQOL total score nearly 3 times the MCID ( $\beta$-coefficient $=-12.4 ; 95 \%$ Confidence Interval= $-18.3,-6.6$ ).

\section{Conclusions}

We found SWD following ABI significantly decreased $\mathrm{HRQOL}$ overall and across multiple health domains. Sleep is potentially modifiable, and our data supports future studies targeting sleep to improve outcomes and HRQOL after ABI. 


\section{Distinguished Poster 1}

\section{Computational Prediction of Targeted Temperature Management Outcome for Personalized Cardiac Arrest Treatment}

Jocelyn J. Hsu, Han B. Kim, Robert D. Stevens

Department of Biomedical Engineering, Johns Hopkins University, Baltimore, MD, United States

\section{Background \& Purpose}

Targeted temperature management (TTM) is associated with improved neurological function in comatose survivors of cardiac arrest. However, the benefits of TTM are not consistently observed and the resource intensive intervention may lead to adverse effects. There is an unmet need to identify patients who are likely to respond to this intervention. The aim of this study is to predict the discharge outcome of postcardiac arrest patients receiving TTM by training a machine leaning (ML) model with physiological and TTM treatment data. It is hypothesized that postcardiac arrest patients most likely to benefit from TTM have a specific data signature.

\section{Methods}

Of 200,859 total patients in the Philips elCU database, 971 received TTM. Only data from the first six hours after ICU admission were analyzed. To identify relationships between features and clinical outcomes, three ML algorithms were used: Generalized linear models (GLM), random forest (RF), and gradient boosting (XGboost). Models were 10-fold cross-validated and resampled 10 times. Models were trained to predict the probability of survival and favorable neurological outcome, measured as motor Glasgow Coma Scale (mGCS) of 6 upon discharge.

\section{Results}

For the prediction of survival, mean AUROC of the three models was 0.687 , sensitivity 0.681 , and specificity 0.583 . For the prediction of favorable neurological function, mean AUROC was 0.654 , sensitivity 0.641 , and specificity 0.569 . The highest ranked features that predicted survival or favorable neurological outcome included male gender, ethnicity, higher mean platelet volume, and higher potassium levels.

\section{Conclusions}

Machine learning algorithms trained with data recorded in the first 6 hours after ICU admission discriminate short-term outcomes in patients receiving TTM after cardiac arrest, and were interpretable. Ongoing model refinement will incorporate other data modalities and involve external validation, with the goal of improving the precision of treatment selection. 


\section{Distinguished Poster 2}

\section{Multimodal Non-invasive Assessment of Intracranial Hypertension}

Fabiano Moulin de Moraes, Gisele Sampaio Silva

Federal University of São Paulo - Department of Neurology and Neurosurgery, São Paulo, Brazil

\section{Background \& Purpose}

Although the placement of an intra-cerebral catheter remains the gold standard method for measuring intracranial pressure (ICP), there are several limitations to the method. This study aimed to estimate the accuracy of four non-invasive methods to assess intracranial hypertension

\section{Methods}

We prospectively collected data on adult intensive care unit (ICU) patients with subarachnoid hemorrhage (SAH), intracerebral hemorrhage (ICH), and ischemic stroke (IS) in whom invasive ICP monitoring had been placed and measures had been simultaneously collected from the following noninvasive indices: optic nerve sheath diameter (ONSD), pulsatility index (PI) using transcranial Doppler (TCD), an 5 point visual scale designed for Computed Tomography (CT) and two parameters (time-topeak [TTP] and P2/P1 ratio) of a non-invasive ICP wave morphology monitor. Intracranial hypertension was defined as an invasively measured ICP $>22 \mathrm{mmHg}$.

\section{Results}

We studied 18 patients ( $\mathrm{SAH}=14 ; \mathrm{ICH}=3 ; \mathrm{IS}=1$ ) on a total of 60 occasions with a median age of 52 years. The median invasively measured ICP was 13 [9.8-16.2] $\mathrm{mmHg}$, and intracranial hypertension was present in 18 occasions (30\%). There was a significant, but diverse correlation between all the noninvasive techniques and invasive ICP (ONSD, $r=0.352 ; \mathrm{PI}, r=0,716 ; C T, r=0.219 ; \mathrm{P} 2 / \mathrm{P} 1$ ratio, $r=0,427$; TTP, $r=0,353-p<0.001$ for all). The area under the curve (AUC) to estimate intracranial hypertension was 0.694 [Cls $=0.62-0.78$ ] for ONSD, 0.750 [95\% Cls 0.69-0.83] for PI, 0,644 [95\% Cis 0.59-069] for CT, 0.786 [95\% Cls 0.72-0.93] for P2/P1 ratio, and 0.694 [95\% Cls 0.60-0.74] for TTP. When the various techniques were combined, the highest AUC (0.861 [0.76-0.93]) was obtained with the combination of $\mathrm{PI}$ and $\mathrm{P} 2 / \mathrm{P} 1$ ratio with an AUC of $0.802(0,72-0,88)$.

\section{Conclusions}

Non-invasive techniques are correlated with ICP and have an acceptable accuracy in estimating intracranial hypertension, with greater accuracy when properly combined. 


\section{Distinguished Poster 3 \\ Polygenic Susceptibility to Hypertension Modifies the Age-of-Onset of Non-Traumatic Subarachnoid Hemorrhage}

Kevin N. Vanent, Julian N. Acosta, Audrey C. Leasure, Rommell B. Noche, Michael R. Levitt, Charles C. Matouk, Murat Gunel, Kevin N. Sheth, Guido J. Falcone

University of Washington, Department of Neurological Surgery, Seattle, WA, United States

\section{Background \& Purpose}

Non-traumatic subarachnoid hemorrhage (SAH) is strongly linked to hypertension, a condition highly influenced by common genetic variants. Following what is known for other complex diseases, we hypothesize that polygenic susceptibility to hypertension is associated with earlier onset of SAH.

\section{Methods}

We analyzed data from the UK Biobank, a large cohort study that enrolled over 500,000 Britons aged 4069. We constructed two polygenic risk scores (PRS) using 664 independent genetic variants known to associate with higher systolic and diastolic blood pressure. We used linear regression to examine the association between these PRS and age of onset for SAH and tested for interaction by sex.

\section{Results}

We evaluated a total of 1,178 SAH cases (mean age, 58 [standard deviation 7]; female sex, 722 [61.3\%]). When evaluating all participants, there was no association between the systolic PRS and age of onset for SAH (adjusted beta, $-0.49 ; 95 \%$ Confidence Interval $[\mathrm{Cl}],-1.19$ to $0.21 ; \mathrm{p}=0.171$ ). However, there was a significant interaction between the PRS and sex $(p<0.001)$. In analyses stratified by sex, each additional standard deviation of the systolic PRS was associated with an earlier onset of SAH in female participants (adjusted beta, $-1.66 ; 95 \% \mathrm{Cl},-2.51$ to $-0.80 ; \mathrm{p}<0.001$ ), but not in male participants (adjusted beta, 1.32; $95 \% \mathrm{Cl}, 0.14$ to $2.51 ; p=0.029$ [Bonferroni-adjusted $p$ value threshold 0.025 for two tests]). Analyses using the diastolic PRS yielded similar results.

\section{Conclusions}

Polygenic susceptibility to hypertension is associated with earlier onset of non-traumatic SAH in women. These results indicate that genetic predisposition may play a more important causal role in younger patients and point to genetic information as possible tools for early identification of high-risk individuals. 


\section{Distinguished Poster 4}

\section{Machine Learning Algorithms of Quantitative EEG for Predicting Outcome after Subarachnoid Hemorrhage}

Hsin-Yi Chen, Sahar F. Zafar, Wei-Long Zheng, Ilayda Top, Jonathan Elmer, Manohar Ghanta, Valdery Moura Junior, Aman B. Patel, Eric S. Rosenthal, Emily J. Gilmore, M. Brandon Westover, Jennifer A. Kim

Yale University, New Haven, CT, United States

\section{Background \& Purpose}

Continuous electroencephalography (cEEG) is used for monitoring after subarachnoid hemorrhage (SAH). Seizures, epileptiform abnormality burden, and quantitative EEG (qEEG) measures such as alpha power predict outcome after $\mathrm{SAH}$. We hypothesized that machine learning methods combining multiple qEEG features improves outcome prediction after SAH.

\section{Methods}

We retrospectively analyzed patients with moderate-to-severe SAH (HH3-5 or FS3-4; 2011-2015) who were monitored with cEEG. Using the Glasgow Outcomes Score (GOS), we dichotomized outcome at discharge into poor (GOS=1-3) and good (GOS=4-5). We extracted eight qEEG features: total power, delta power, theta power, alpha power, alpha delta ratio, percent alpha variability, Shannon entropy, and epileptiform discharge (ED) burden (defined as the number of sporadic or periodic discharges detected per hour). We used Persyst to identify EDs and MATLAB to calculate all features. We trained L1-regularized (LASSO) regression and Random Forest (RF) models to predict outcome at discharge and 3 months. We evaluated performance using 5 -fold cross validation.

\section{Results}

113 patients met study criteria; 91 (80.53\%) had poor outcome at discharge. The RF (AUC=0.96) model using qEEG features from the first $72 \mathrm{~h}$ post-SAH outperformed the baseline logistic regression of $\mathrm{HH}$ and age ( $A \cup C=0.83$ ). The addition of clinical variables did not improve the model ( $A \cup C=0.95$ ). The best LASSO model included qEEG and clinical features but performed similarly to the baseline model (AUC=0.83).At 3-months follow up, 105 patients had data; $48(45.71 \%)$ had poor outcome. Both LASSO $(A \cup C=0.75)$ and $R F(A \cup C=0.75)$ qEEG models outperformed the baseline model ( $A \cup C=0.73)$. Both models were improved by the addition of clinical variables (LASSO AUC $=0.78, \mathrm{RF} A \cup C=0.80$ ). However, none of these differences were significant.

\section{Conclusions}

Automated calculations of qEEG features can be used in LASSO and RF models to predict SAH outcome. RF models including qEEG with or without clinical variables performed the best. This work needs to be validated in independent cohorts. 


\title{
Distinguished Poster 5
}

\section{Assessment of Trainees' Performance During a Simulation of Viral Encephalitis Complicated by Status Epilepticus}

\author{
Melissa B. Pergakis, WanTsu Wendy Chang, Camilo A. Gutierrez, Benjamin Neustein, David Lerner, \\ Nicholas A. Morris
}

University of Maryland Medical Center, Neurocritical Care, Program in Trauma, Baltimore, MD, United States

\section{Background \& Purpose}

Delays in treatment of both Herpes Simplex Virus (HSV) encephalitis and seizures are associated with poor patient outcomes. We sought to assess trainee performance in the management of HSV encephalitis complicated by status epilepticus using high-fidelity simulation.

\section{Methods}

In this prospective, observational, single-center simulation-based study, participants ranging from interns to fellows managed a patient with encephalopathy and fever due to HSV encephalitis followed by non-convulsive status epilepticus. The case and critical actions were developed by a modified Delphi approach and based on the Neurocritical Care Society's Emergency Neurological Life Support (ENLS) protocols. The primary outcome measure was the critical action sum score. We sought validity evidence to support our findings by comparing novice, intermediate, and advanced trainees' performance using ANOVA.

\section{Results}

Fifty-one participants completed the case. The mean sum of critical actions completed was 14.7/25 (59\%). Eighty-one percent of participants administered an appropriately-dosed benzodiazepine, $94 \%$ administered an appropriately-dosed 2 nd line anti-seizure drug, but only $67 \%$ recognized non-convulsive status epilepticus on continuous electroencephalogram and started an anesthetic. Despite $73 \%$ of trainees obtaining a lumbar puncture, only $46 \%$ recognized viral encephalitis as the most likely diagnosis with $23 \%$ starting appropriate treatment. There was a significant effect of training level on critical action sum score (novice mean score [standard deviation $(S D)]=12.5(2.4)$ vs. intermediate mean score $(S D)=14.6$ (2.7) vs. advanced mean score (SD) 17.7 (3.4), $p<.001$ ). Sum scores were positively correlated with multiple choice pre-test scores, $r=.448 p<.01$, and self-rated experience with status epilepticus, $r=.358, p<.05$.

\section{Conclusions}

Trainees frequently fail to diagnose and treat HSV encephalitis despite its textbook presentation. Most trainees are proficient in the initial management of status epilepticus, but fewer adequately manage refractory status epilepticus. Our findings warrant further study to understand why classic cases escape trainee diagnosis. 


\title{
Distinguished Poster 6
}

\section{Exposure to Non-lung Protective Mechanical Ventilation is Associated with Outcome in Critically III Patients with Acute Brain Injury: A Machine Learning Approach}

\author{
Neha Sangana, Hanbiehn Kim, Robert D. Stevens
}

Johns Hopkins University, Baltimore, MD, United States

\section{Background \& Purpose}

Patients with acute brain injury ( $\mathrm{ABI}$ ) commonly receive mechanical ventilation (MV) which can be harmful due to ventilator-induced lung injury. Our aim is to explore relationships between MV and outcome of patients with ABI. We hypothesize that MV variables are important determinants of clinical outcomes.

\section{Methods}

The eICU database was searched for patients with traumatic brain injury (TBI) or stroke who were mechanically ventilated. A set of features was crafted based on current recommendations for lungprotective ventilation and included time "out-of-range" (OOR) for tidal volume per ideal body weight ( $>8$ $\mathrm{ml} / \mathrm{kg}$ ), plateau pressure ( $>30 \mathrm{cmH} 2 \mathrm{O})$, PEEP ( $<5 \mathrm{cmH} 2 \mathrm{O})$. Two models were created: (1) A clinical model which uses only the first value recorded for each feature; and (2) A combined model which includes predefined OOR MV variables. Outcomes were defined as "Unfavorable" for patients who died or whose discharge motor Glasgow Coma Score (mGCS) was < 5, and "Favorable" for patients who were alive and had a $m G C S \geq 5$ at discharge. Three different meachine learning models (generalized linear model, $X G B o o s t$, and random forest) were trained using extracted features.

\section{Results}

Data were identified for a total of $839 \mathrm{TBI}$ and 1,221 stroke ICU stays in which patients were mechanically ventilated. The best performing classifier was a random forest model. Clinical and combined models had an AUROC of $0.67 \pm 0.07$ and $0.79 \pm 0.13$ respectively for Stroke and an AUROC of $0.70 \pm 0.07$ and $0.81 \pm 0.07$ respectively for TBI. As shown in Figure 1 , a number of OOR MV variables and respiratory physiologic variables contributed to the prediction of outcome. Duration of exposure to Out of Range (OOR) tidal volume, PEEP and plateau pressure were identified as top contributors with longer OOR durations predictive of unfavorable outcome.

\section{Conclusions}

These results suggest that exposure to non-lung protective MV may contribute to clinical outcomes in mechanically ventilated stroke and TBI patients. 


\section{Distinguished Poster 7}

\section{Acute Traumatic Brain Injury Magnetic Resonance Imaging Lesions Associated with Paroxysmal Sympathetic Hyperactivity Diagnostic Likelihood}

Jamie Podell, Gunjan Parikh, Serenity Miller, Hemantkumar Tripathi, Jiachen Zhuo, Steven Roys, Melissa Motta, Nicholas Morris, Wan-Tsu Chang, Gary Schwartzbauer, Peter Hu, Rao Gullapalli, Neeraj Badjatia

University of Maryland School of Medicine, Department of Neurology, Program in Trauma, Baltimore, $\mathrm{MD}$, United States

\section{Background \& Purpose}

Paroxysmal sympathetic hyperactivity (PSH) is a dramatic syndrome of autonomic dysfunction that is associated with worse outcomes after traumatic brain injury (TBI), but its neural underpinnings are not well-defined. We investigate whether traumatic lesions within the central autonomic network are associated with higher PSH diagnostic likelihood.

\section{Methods}

We performed a retrospective study of critically ill acute TBI patients who were admitted for at least 14 days and underwent a brain MRI as part of clinical care from January 2016 to July 2018. We tabulated PSH Assessment Method (PSH-AM) Diagnostic Likelihood Tool (DLT) scores for each patient and manually reviewed MRIs for SWI, FLAIR, and ADC lesions in pre-defined brain regions of interest. DLT scores were compared according to regional lesion presence using the Wilcoxan Ranked sum test. A multiple regression analysis controlling for epidemiologic features including GCS, age, and sex was also performed in order to identify independent imaging predictors of PSH diagnostic likelihood.

\section{Results}

Inclusion criteria were met by 121 patients, of whom 60 were clinically diagnosed with PSH, and median DLT score was 5 (range 1-11). MRI Brain was performed on median hospital day 5 (range 0-30). Significantly higher DLT scores were observed in patients with lesions in the corpus callosum (SWI, FLAIR, ADC), medial temporal gyrus (SWI, FLAIR), medial prefrontal/anterior cingulate cortices (SWI, FLAIR), ventral diencephalon (SWI), thalamus (SWI), and bilateral hemispheric white matter (SWI), while there were no imaging associations with DLT scores in the brainstem or insula. SWI lesion presence in the corpus callosum ( $p=0.003$ ) was identified as the only independent imaging predictor of PSH diagnostic likelihood.

\section{Conclusions}

Our preliminary work supports disconnection models of PSH, where structural disruption of white matter tracts contributes to pathologic dysregulation of the autonomic nervous system. Future quantitative imaging and physiologic studies are warranted. 


\title{
Distinguished Poster 8
}

\section{A Robust Data Archive Format for Traumatic Brain Injury Physiology and Machine Learning}

\author{
D. Habboush, E. Rosenthal, B Foreman, Z. Goldblum, P. Smielewski, D. Moberg
}

School of Biomedical Engineering, Science and Health Systems, Drexel University, Philadelphia, PA, United States

\section{Background \& Purpose}

In TBI patient management, a common need is for a data format that will accommodate the highresolution multimodal data and metadata, complete research, and share data among trials. In this abstract we discuss our work to develop a robust data format that combines diverse data types, provides essential metadata, and permits rapid real-time analytics.

\section{Methods}

We were able to develop several tools to convert high-resolution physiological data and metadata into an HDF5 file format and interact with the data. We created an HDF5 converter which converts multiple binary data files into one single, readable file, removing many of the limitations surrounding big data analysis and we integrated it with our IBM cloud platform. We also developed a real-time HDF5 converter which converts high-resolution data and appends it to a continuously growing HDF5 file to create real-time visualizations and analytics. We've also published an open-source HDF5 Python package to interact with the HDF5 files.

\section{Results}

The conversion process was tested using the patient data files from multiple studies and testing sites. Over 257 patient files from the Transforming Research and Clinical Knowledge in Traumatic Brain Injury (TRACK-TBI) study were converted into the HDF5 format amounting to around 742 GB of data. The HDF5 converter and the Real Time Converter were validated through manual byte conversions which ensured the number of data points stored in the newly created HDF5 file matched the original binary data file size.

\section{Conclusions}

In this work, we were able to develop several mechanisms to convert multiple files of high-resolution data into a single formatted HDF5 file ready for analysis. With our tools data can be analyzed in realtime and the results can also be stored directly into the HDF5 file. A common data format will facilitate TBI research across multiple trials. 


\section{Distinguished Poster 9 \\ Role of Plasma Lipid Metabolites after Mild Traumatic Brain Injury}

Aaron M. Gusdon, Jude Savarraj, Atzhiry Paz, Glenda Torres, Anthony N. Moore, John B. Redell, H. Alex Choi, , Pramod Dash

University of Texas Health Science Center, Department of Neurosurgery, Houston, TX, United States

\section{Background \& Purpose}

Lipids play a critical role in brain physiology and cell signaling and are critical for cell membrane repair after injury. We hypothesized that levels of plasma lipids would be significantly altered after mild traumatic brain injury (TBI), providing insight into the pathophysiology of injury and a biomarker for outcomes.

\section{Methods}

Untargeted mass spectrometry-based metabolomics analysis was performed for 84 subjects with mild $\mathrm{TBI}$ and 39 healthy volunteers. Of the TBI subjects, 32 had paired samples at early and late (6-month) time points. Fold changes of each metabolite were determined between control and TBI subjects and within the TBI group between early and late time points. Bioinformatics analyses included hierarchical clustering algorithms to assess correlations between groups of metabolites and principal component analysis (PCA). Extended Glasgow Outcome Scale (GOSE) and modified Rankin scale (mRS) scores were collected at discharge and 6-monhs. Univariate models were created to determine the relationship between each metabolite and functional outcomes, with multivariate models created subsequently.

\section{Results}

Early after injury, phosphatidylcholines (PC), lysophospholipids, acylcholines, glycerophosphorylcholine, and phosphocholine were decreased, while diacylglycerols (DAG), phosphatidylethanolamine (PE), long chain saturated and monounsaturated fatty acids were increased. Downregulated metabolites normalized at 6-months in paired samples, while DAG, PE, and fatty acids remained increased at 6months. PCA accounting for differentially regulated lipids resulted in significant segregation between control and TBI subjects. Higher levels of one particular PC (18:0/18:2w6) were associated with better functional outcomes at discharge (OR 5.78 [95\% Cl 1.20-20.9]) and 6-months (OR 13.2 [95\% Cl 4.5651.9]). Subjects whose levels of this PC increased comparing early and 6-month time points had improved functional outcomes.

\section{Conclusions}

Plasma lipids are differentially regulated after TBI. Lower PC levels occurred early after TBI and were associated with outcomes. PCs may be important for membrane repair after TBI and may represent an important TBI biomarker. 


\section{Distinguished Poster 10}

\section{Use of a Pharmacologic Neurostimulation Bundle in Patients with Traumatic Brain Injury at a Level II Trauma Center}

Martin Faile, Sara Kutner, Kirsten Busey

Sarasota Memorial Hospital Department of Pharmacy, Sarasota, FL, United States

\section{Background \& Purpose}

Traumatic brain injury (TBI) is a prevalent cause of morbidity and mortality in the United States. During the subacute phase of recovery, neurotransmission pathways in the brain are disrupted resulting in decreased levels of consciousness, cognitive impairment, and behavioral disorders. Growing evidence suggests that pharmacologic neurostimulation may improve patient outcomes. Since 2016, we have utilized a three-drug regimen of amantadine, sertraline, and donepezil in post-TBI patients. The purpose of this study was to describe patient outcomes and safety associated with the use of this neurostimulation bundle.

\section{Methods}

This institutional review board approved, single group, retrospective, descriptive chart review study included adult patients admitted with a severe TBI who received the neurostimulation medication bundle for at least 72 hours. Exclusion criteria included patients taking amantadine, sertraline or donepezil prior to admission, patients with a history of stroke, dementia, Parkinson's disease, or other movement disorders. The primary outcome measure was improvement in GCS from prior to neurostimulation bundle initiation to discontinuation or completion of the bundle at 30 days. Secondary outcomes included incidence of adverse drug reactions, and time from bundle initiation to participation with physical therapy.

\section{Results}

A total of 72 patients were included in the study with a median age of 46 years old $(18,76)$. Forty-two patients (65\%) had an improvement in GCS after bundle initiation, with a median improvement in score of $4(2,5)$. For adverse events, $11.1 \%$ of patients experienced hyponatremia, $6.9 \%$ had agitation, $2.9 \%$ had QTc prolongation, and $6.9 \%$ had reported seizure activity. Six patients had the bundle discontinued early due to adverse effects potentially attributed to the bundle. Time from bundle initiation to participation in physical therapy was a median of two days $(0,6.5)$.

\section{Conclusions}

Use of a neurostimulation bundle in patients with severe TBI appears to be safe with a low incidence of adverse effects. 


\section{Distinguished Poster 11}

\section{A Computational Model to Predict Discharge Responsiveness in Non-Neurological ICU Patients}

Haoyin Xu, Jacob Desman, Munachiso A. Igboko, Qianqi Huang, Zixuan Wang, Alisha Kodibagkar, Kirby Gong, Raimond L. Winslow, Joseph L. Greenstein, Robert D. Stevens

Johns Hopkins University, Biomedical Engineering, Baltimore, MD, United States

\section{Background \& Purpose}

Though commonly associated with a primary neurological disorder, states of decreased responsiveness are frequently seen in non-neurological ICU patients. Here, we build and validate a computational model to predict the discharge responsiveness phenotype in this population.

\section{Methods}

The eICU and MIMIC-IV datasets were used for train-and-test and external validation, respectively. We selected adult patients whose ICU length of stay was $\geq 2$ and $\leq 7$ days, and only data collected in the first $24 \mathrm{~h}$ following ICU admission were used. Responsiveness was evaluated on ICU admission and discharge using the motor subscore of the Glasgow Coma Scale (mGCS), with responsive and unresponsive states defined as $\mathrm{mGCS}=6$ and $\mathrm{mGCS}<6$, respectively. Binary classifications were trained on the full population and on the subsets who were responsive on admission (RA), and unresponsive on admission (UA). The final feature space contained demographics, physiological signals, lab values, and medications. A gradient boosted (GB) decision tree model was selected as the classifier. After 5 -fold cross validation, model performance was determined by area under the receiver operating characteristic (AUROC) analysis.

\section{Results}

The final dataset consisted of 37,568 stays for eICU and 20,127 stays for MIMIC-IV. Model discrimination expressed as AUROC(SD) was 0.86 (0.01), 0.84 (0.01), and 0.79 (0.01) for the entire sample, RA subset and UA subset, respectively. Features strongly associated with unresponsiveness at discharge were higher heart rate, lower systemic blood pressure, higher blood urea nitrogen and higher red blood cell count.

\section{Conclusions}

In patients admitted to the ICU with a non-neurological diagnosis, a machine learning model trained with data collected in the first 24 hours of ICU stay accurately predicts responsiveness at discharge. These predictions were interpretable and could inform strategies to prevent neurological deterioration or enhance neurological function during critical illness. 


\title{
Distinguished Poster 12
}

\section{Using Contextual Data to Enhance Machine Learning in Traumatic Brain Injury}

\author{
Z. Goldblum, D. Olson, E.S. Rosenthal, B. Foreman, D. Moberg
}

School of Biomedical Engineering, Science and Health Systems, Drexel University, Philadelphia, PA, United States

\section{Background \& Purpose}

The influence of a variety of contextual factors on recorded physiologic data, such as intracranial pressure (ICP), in patients with traumatic brain injury (TBI) has been detailed in the literature. Efforts to develop artificial intelligence paradigms to detect neuroworsening or other changes in patient state and prediction of significant clinical events have not included the contextual factors necessary to ensure validity of bedside physiologic measurements; despite a likelihood that this will aid in the success of the analytic methods. We focused on one such contextual factor: whether the ICP value from an extraventricular drain (EVD) was recorded when not connected to the pressure transducer.

\section{Methods}

We created a system that generates contextual metadata annotations time-synchronous with physiologic data. We designed a novel sensor device that determines the position of an EVD stopcock. A microcomputer calculates and records the positional data to create annotations in real-time. Four hours of simulated clinical use data $(n=864,000)$ was collected to evaluate accuracy.

\section{Results}

Our system successfully integrated the sensor data stream transmitted via Bluetooth and generated correct, time-synchronized stopcock position annotations for all 864,000 data points. Dimensions of the stopcock sensor device were deemed acceptable by clinical reviewers.

\section{Conclusions}

We were successful in getting an initial and important piece of the contextual metadata, the EVD stopcock position, synchronized with the physiology. These annotations identify valid or invalid/artifactual segments of ICP data which may improve machine learning decoding accuracies. Our system supports the integration of future data sources with connections to 10 Bluetooth devices and 4 serial devices. The goal is to add other environmental sensors (e.g., head of bed, noise level, etc.) and explore the effect of other contextual factors on physiologic data. 


\title{
Distinguished Poster 13
}

\section{Percutaneous Ultrasound Gastrostomy (PUG) for Neurointensivists: No More Forcing Square PEGs in Round Holes}

\author{
Amay Parikh, Gilbert Mbeo, Mohammed Zia Khan, Sima Patel, Bassel Raad, Okorie N. Okorie \\ AdventHealth Orlando, Department of Critical Care, Neurocritical Care Division, Orlando, FL, United \\ States
}

\section{Background \& Purpose}

For patients with severe neurologic conditions, long-term mechanical ventilation and enteral nutrition necessitates placement of tracheostomy and gastrostomy feeding tubes. The benefits of concomitant placement have been reported but this approach is underutilized because many specialists cannot perform both procedures. A new method for gastrostomy placement, Percutaneous Ultrasound Gastrostomy (PUG), was adopted by our neurointensivists. The purpose of this study was to determine if concomitant placement of tracheostomy and gastrostomy tubes improved efficiencies of care in our neurocritical care unit (NCCU).

\section{Methods}

Retrospective cohort study in a 28-bed Level I NCCU at a quaternary care hospital. Patients admitted to the NCCU in calendar year 2019 who underwent percutaneous dilational tracheostomy (PDT) and bedside endoscopic gastrostomy (PEG) were compared with patients receiving PDT and bedside PUG under trial from May to July 2020. Time to gastrostomy placement and ICU length of stay (LOS) were compared between PEG and PUG groups.

\section{Results}

9 PUG and 41 PEG procedures performed in the NCCU were reviewed. All PUGs were performed by neurointensivists. 89\% (8/9) of PUG procedures were completed concomitant to PDT compared to $0 \%$ (0/41) of PEG. Average time to PUG tube placement from indication was significantly shorter than PEG ( 0.5 vs. 2.5 days, $p=0.005$ ). In patients receiving tracheostomy following 7 days of admission, ICU LOS was significantly shorter with PUG than PEG (13.8 vs. 21.5 days, $p=0.047)$.

\section{Conclusions}

PUG decreases gastrostomy tube placement delays and ICU LOS in the NCCU. Neurointensivists skilled in tracheostomy and ultrasound can independently perform concomitant PDT and PUG at their discretion, including weekends and holidays. Concomitant PDT and PUG reduces anesthesia and care team size by requiring only one instance of sedation/paralytics and eliminating the need for scheduling other hospital team services. 


\title{
Distinguished Poster 14
}

\section{Anti-Epileptic Drug Use in Subarachnoid Hemorrhage: Preliminary Findings}

\author{
Sarah E. Nelson, Vishank Shah, Salia Farrokh, Jose I. Suarez
}

Departments of Neurosurgery and Neurology, Mount Sinai West, New York, NY, United States

\section{Background \& Purpose}

Despite published guidelines, anti-epileptic drugs (AED) are not used consistently in subarachnoid hemorrhage (SAH), and data guiding their management are not clear. We sought to determine whether variations in practice and outcomes occur with respect to AEDs prescribed to SAH patients.

\section{Methods}

Using the Vizient Clinical Data Base/Resource ManagerTM, we analyzed demographics, AED use, and outcomes of patients diagnosed with nontraumatic SAH from quarter 4, 2015 to quarter 2, 2020. We compared patients who received AEDs versus those who did not. We further analyzed patients who received AEDs by geographic region (Northeast, Midwest, South, West).

\section{Results}

A total of 50,511 nontraumatic SAH patients were evaluated. Of these, 44,222 received AEDs; 6,296 did not. Patients receiving AEDs were younger ( $59.4 \pm 15.5$ versus $65.4 \pm 16.6$ years, $p<0.0001$ ), were more likely to be female $(57.4 \%$ versus $55.8 \%, p=0.022)$, and incurred greater hospital costs as well as intensive care unit and hospital lengths of stay. However, they were less likely to die while hospitalized $(19.7 \%$ versus $28.4 \%, p<0.001)$. Among those who received AEDs, there were no differences by sex but Northeast patients were older than in other regions. AED type was significantly different by region $(p<0.001)$ with a trend for increased levetiracetam use in the Northeast and more pregabalin/gabapentin use in the West. In-hospital mortality was different overall by region; West patients had the highest (21.2\%; $p=0.004)$. Intensive care unit and hospital lengths of stay and hospital costs were greater for patients in the West than in other regions (all $p<0.0001$ ).

\section{Conclusions}

Most nontraumatic SAH patients received AEDs and tended to have protracted stays but lower in-house mortality versus those who did not receive AEDs. AED type varied by region. SAH patients on AEDs treated in Western hospitals had longer hospital stays. Additional data including multivariable analyses are needed to confirm these findings. 


\section{Distinguished Poster 15}

\section{Improvements in Education of Surrogate Decision-makers in the Neuroscience ICU are Needed}

Elizabeth Carroll, Julie Giles, Ariane Lewis

NYU Langone Health, New York, NY, United States

\section{Background \& Purpose}

Data on surrogate comprehension of a patient's clinical condition in the neuroscience ICU is limited.

\section{Methods}

We prospectively surveyed surrogates of patients admitted to the neuroscience ICU for $>48$ hours from 10/2018-3/2021 who lacked decision-making capacity. The survey investigated surrogate communication with the healthcare team and understanding of the patient's clinical condition. A member of the healthcare team evaluated how well they perceived the surrogate understood the situation and provided clinical data.

\section{Results}

We enrolled 28 surrogates ( $26 \%$ children, $19 \%$ spouses, $15 \%$ parents, $41 \%$ other) a median of 10 (interquartile range 5-16) days after hospital admission. All surrogates had spoken directly to a clinician during the patient's hospitalization, and $89 \%$ reported that a physician or advanced practice provider was their primary source of clinical information. Half (50\%) of surrogates felt their overall understanding of the hospitalization was "excellent," but $4 \%$ felt it was "poor." The healthcare team felt that $39 \%$ of surrogates had "excellent" understanding and 7\% had "poor" understanding. There was slight to no agreement between surrogate and team perception of surrogate understanding $(k=0.172)$; surrogates and clinicians rated the surrogate's overall understanding the same in only $50 \%$ of cases. With increasing SAPS II score, team perception of surrogate understanding became significantly worse $(p=0.011)$, and self-rated understanding was non-significantly worse $(p=0.127)$. There was no significant relationship between self or team perception of surrogate understanding and age, education, relationship, or hospitalization day.

\section{Conclusions}

Although the healthcare team and surrogates do not have concordant views on surrogate understanding of a patient's clinical condition in the neuroscience ICU, increasing acuity of illness is associated with perception of worse surrogate understanding. Additional research is needed to identify means to improve surrogate comprehension. 


\section{Distinguished Poster 16}

\section{Serial Multimodal Functional Brain Network Evaluation in Children with Acute Disorders of Consciousness, Including Coma and Covert Consciousness: A Novel Demonstrative Case Series}

Varina L. Boerwinkle, Bethany Sussman, Josh Koch, Sarah Wyckoff, Maria J. Gonzalez, Tara Mangum Barrow Neurological Institute at Phoenix Children's Hospital, Phoenix, AZ, United States

\section{Background \& Purpose}

Coma and disorder of consciousness (DOC) are common manifestations in the setting of acute brain conditions in children. To begin to bridge this gap national and international DOC guidelines propose serial multimodal brain network evaluation (MBNE) in acute DOC, which although recommended in children is not reported. We therefore evaluated the feasibility and utility of serial MBNE in acute DOC in children.

\section{Methods}

Serial MBNE, occurred in the acute phase in the ICU and later repeated, without sedation, in three pediatric unresponsiveness patients between April 2017 and April 2021, age 7 to 15 years. Tests included continuous video electroencephalogram ( $V E E G)$, visual evoked responses, somatosensory evoked responses, brainstem auditory evoked response (BEAR), developmentally appropriate active and passive task-based functional MRI (tb-fMRI), and resting state fMRI ( $r s-f M R I)$. Tb-fMRI included passive movement of the hand, passive checkerboard visual stimulation, active command to move the hands, legs, repeat words, and imagine common animals. Results were implemented into care and outcomes were collected.

\section{Results}

MBNE were acquired initially on days ranging 2-15, and again on day 10-48 (except one rs-fMRI at day 254). The initial exams were similarly unresponsive, and in the acute phase redirection toward comfort measures was discussed with families. The serial MBNE reflected covert consciousness in two subjects with pathological signal indicative of deep source seizure, which was treated with temporal improvement in both clinical and MBNE findings. One of these patients slowly recovered completely over a years-time, and the other remains in locked-in state with vertical eye movements to command. The remaining patient had mixed MBNE findings initially with present brain networks and pathological connectivity. These findings led to increased testing then treatment for vasospasm, which was only partially successful, and he sustained further brain destruction. Repeat MBNE was commiserate with the deterioration reflecting brain network deterioration and lack of potential for significant recovery. He remains in unresponsive wakefulness condition. Determinations to escalate therapy, recommend supportive care, and re-offer recommendation to redirect toward comfort measures were influenced by the serial MBNE.

\section{Conclusions}

Guideline supported serial MBNE in children with acute DOC is feasible and allows for improved DOC endotyping, illumination of treatment opportunities, influences care team acute phase direction of care determinations, impacts outcomes, and increases standardization of care in this demonstrative case series. 


\section{Distinguished Poster 17}

\section{Automated Pupillometry and Prediction of Electroencephalography Reactivity in Critically III Patients}

Lorenzo Peluso, Lorenzo Ferlini, Marta Talamonti, Narcisse Ndieugnou Djangang, Elisa Gouvea Bogossian, Marco Menozzi, Filippo Annoni, Paolo Severgnini, Jacques Creteur, Mauro Oddo, Jean-Louis Vincent, Nicolas Gaspard, Fabio Silvio Taccone

Department of Intensive Care, Erasme University Hospital, Brussels, Belgium

\section{Background \& Purpose}

The aim of this study was to evaluate whether parameters derived from an automated pupillometer (AP) might help to assess the degree of cerebral dysfunction evaluated with electroencephalography (EEG) in critically ill patients.

\section{Methods}

In this prospective study, we performed a pupillary assessments using the AP in three subgroups of patients, concomitantly monitored with continuous EEG: i) "post-anoxic brain injury"; ii) "primary brain injury"; iii) "others" (i.e. sepsis, liver failure, etc.). A neurologist scored the degree of encephalopathy and reactivity on EEG using a standardized scale. The mean value of Neurologic Pupil Index (NPi), pupillary size, constriction rate, constriction and dilation velocity (CV and DV) and latency for both eyes, obtained using the $\mathrm{NPi}^{\circledR}-200$ (Neuroptics, Laguna Hills, CA, USA), were reported.

\section{Results}

We included 214 patients in the analysis, EEG tracings were categorized as: mild ( $n=111,52 \%$ ), moderate $(n=65,30 \%)$ or severe $(n=16,8 \%)$ encephalopathy; burst-suppression $(n=19,9 \%)$ or suppression background ( $n=3,1 \%)$; a total of $38(18 \%)$ EEG were classified as "unreactive". We found a significant difference in all pupillometry variables among different EEG categories. Moreover, an unreactive EEG was associated with lower NPi, pupil size, pupillary reactivity, CV and DV and a higher latency than reactive recordings. Low DV (Odds ratio 0.01 [95\% confidence intervals $0.001-0.15$ ]; $p<0.01$ ) was independently associated with an unreactive EEG, together with the use of analgesic/sedative drugs and high lactate concentrations. In particular, DV values had an area under the curve (AUC) of 0.85 [0.78$0.91 ; p<0.05$ ] to predict the presence of unreactive EEG. In subgroups analyses, AUC of DV to predict unreactive EEG was lower (0.70 [0.55-0.86]) in post-anoxic brain injury than primary brain injury $(0.91$ $[0.82-1.00 ; p<0.01])$ and other diseases (0.95 [0.88-1.00; $p<0.01])$.

\section{Conclusions}

DV measured by the AP might effectively identify an unreactive EEG background, particularly in patients without anoxic brain injury. 


\section{Distinguished Poster 18}

\section{Variability of Post-Cardiac Arrest Neuroimaging Practices in Pediatric Intensive Care Units}

Natalie UlIman, Craig A. Press, Chella A. Palmer, Kellimarie K. Cooper, Robert A. Berg, Stuart H. Friess, Kathryn Graham, Todd J. Kilbaugh, Ryan W. Morgan, Peter M. Mourani, Vinay M. Nadkarni, Maryam Y. Naim Danna W. Qunibi, Ron W. Reeder, Heather A. Wolfe, Robert M. Sutton, Matthew P. Kirschen, on behalf of the ICU-RESUS Investigators and the Collaborative Pediatric Critical Care Research Network Investigators

The Children's Hospital of Philadelphia, Department of Neurology, Philadelphia, PA, United States

\section{Background \& Purpose}

Neuroimaging is performed after cardiac arrest to establish arrest etiology, guide neuro-resuscitative management, and assist with prognostication. The objective of this study was to characterize the epidemiology of neuroimaging performed after cardiac arrest in pediatric intensive care units (PICUs).

\section{Methods}

This was a planned secondary analysis of an ongoing large prospective multi-center interventional trial of in-hospital cardiac arrest (ICU-Resuscitation Project, NCT02837497). Patients who achieved return of circulation (ROC) were eligible for inclusion. The association between patient demographics, cardiac arrest characteristics, and post-arrest factors and whether a neuroimaging study was obtained was evaluated using Wilcoxon rank-sum tests. Multivariable regression was used to identify patient characteristics associated with increased odds of obtaining neuroimaging.

\section{Results}

Of 418 children who achieved ROC, 111 (27\%) underwent neuroimaging within 7 days of arrest. Of those with neuroimaging, 49\%, 23\%, and $47 \%$ underwent head CT, brain MRI, and head ultrasound, respectively. There was substantial variability in the incidence, modality, and timing neuroimaging across sites. CTs were obtained both $<24$ hours $(41 \%)$ and $>24$ hours $(60 \%)$ post-arrest, whereas $82 \%$ of MRIs were obtained $>24$ hours post-arrest. Severity of illness pre-arrest was higher in patients who underwent CT (median PRISM $5.5[1.0,14.0]$ vs $1.0[0.0,5.0], p=0.001)$ but not MRI $(0.5[0.0,8.0]$ vs 3.0 $[0.0,9.0], N S)$. In multivariate analysis, factors that increased the odds of obtaining a CT were patient age $>1$ month, study site, malignancy, and CPR duration $>6$ minutes (all $p<0.01$ ). Factors that increased the odds of obtaining an MRI were respiratory insufficiency prior to arrest, higher baseline functional status, lower vasoactive infusion score prior to arrest, and CPR duration 6-35 minutes (all $p<0.05$ ).

\section{Conclusions}

In this large, prospective study of pediatric in-hospital cardiac arrest, post-arrest neuroimaging was highly variable between institutions, and both patient and cardiac arrest characteristics played a significant role in neuroimaging selection. 


\title{
Distinguished Poster 19
}

\section{Correlation of Detection of B-waves in Intracranial Pressure Signals Between Invasive and Non- invasive Methods}

\author{
Joao A. Gomes, Catherine Hassett, Nina Moore, Danilo Cardim \\ Neurointensive aare unit, Cleveland Clinic, Cleveland, $\mathrm{OH}$, United States
}

\section{Background \& Purpose}

Following brain injuries, the patterns of intracranial pressure (ICP) waveforms may reflect pathological processes that ultimately lead to unfavorable outcomes. In particular, ICP slow waves (SW - frequency range: $0.005-0.05 \mathrm{~Hz}$ ) magnitude has been shown to reflect dynamic oscillations in cerebral blood volume associated with autoregulatory cerebral vasodilation/vasoconstriction. Our goals were to evaluate the presence of ICP SW activity following acute brain injury, and to examine the relationship between SW activity monitored using invasive ICP and non-invasive (nICP) methods.

\section{Methods}

We retrospectively reviewed ICP (external ventricular drain) and nICP (mechanical extensometer Brain4Care Corp.) data collected in adult brain-injured patients admitted to the Neurointensive Care Unit between $01 / 2021$ and $04 / 2021$. The time series from both signals were first treated to remove movement artifacts followed by detrending of the signals using a low-pass finite impulse response filter of $0.015 \mathrm{~Hz}$. Power spectral density analysis was performed using a passband FFT filter set to 0.005-0.05 $\mathrm{Hz}$. We then calculated the total power (representative of the SW amplitude) in the frequency domain and evaluated the relationship between SW detection with invasive and non-invasive methods using repeated measures correlation coefficient analysis. Data were analyzed using ICM+ and RStudio software platforms.

\section{Results}

Ten patients were identified. Mean age was $46+18$ yrs., and hemorrhagic stroke was the most common etiology (80\%). All were undergoing mechanical ventilation and $7 / 10$ were sedated. Median (IQR) GCS: 10 (2.75). The SW correlation between ICP and nICP was strong, R=0.72 (95\% Cl: 0.65-0.77) $(p<0.0001)$.

\section{Conclusions}

Slow wave activity can be detected reliably both by conventional invasive ICP as well as a novel nICP device, with strong correlation. Further study of the applications of this novel clinical tool is warranted with the goal of intervening early to improve neurologic outcome in acute brain injuries 


\section{Distinguished Poster 20}

\section{Dynamic Prediction of Delayed Cerebral Ischemia in Aneurysmal Subarachnoid Hemorrhage}

Emanuela Keller, Jens M. Boss1, Jan Willms1, Corinne Inauen, Achim Müller, Laura P. Westphal, Stefan Bögli, Carl I. Muroi, Christian Strässle, Emanuela Keller1 shared first authorshipNeurocritical Care Unit, Dept. of Neurosurgery and Inst. of Intensive Care Medicine, University Hospital Zurich, Switzerland

Neurocritical Care Unit, Dept. of Neurosurgery and Inst. of Intensive Care Medicine, University and UniversHospital Zurich, Switzerland

\section{Background \& Purpose}

Outcome prediction after aneurysmal subarachnoid hemorrhage (aSAH) is based on scores, which are determined once at admission. However, the occurrence of delayed cerebral ischemia (DCI) depends on multiple concomitant and continuously changing factors. The goal of this study is to establish an automated analysis pipeline to predict DCI from multimodal data.

\section{Methods}

Multimodal data (patient characteristics, imaging, and laboratory values) from 143 patients with aSAH were analyzed. DCl was defined as neurological worsening, new ischemia and/or perfusion deficits in native or contrast-enhanced CT/MRI. To capture the temporal dynamics leading to $\mathrm{DCl}$ onset, patient's data were aligned using the first $\mathrm{DCl}$ event as an anchor. Gini importance and SHAP values were used to identify the 10 most informative baseline characteristics and laboratory values, respectively. The two models (static and dynamic) were combined to a voting model. For DCl prediction in all three models, random forest machine learning algorithms were applied, and 5-fold cross-validation was used to estimate model performance. The usability was further illustrated by a leave-one-out algorithm simulation starting from ICU admission until day 14.

\section{Results}

68 among 143 patients developed $\mathrm{DCl}(48 \%)$. In the ROC analysis the voting model performed best with an AUC of $0.747+0.094$, followed by the dynamic and the static model with an AUC of $0.729+0.083$ and $0.679+0.131$ respectively. Leave-one-out simulation experiments applying the voting model showed a highly dynamic probability for $\mathrm{DCl}$ occurrence over the course of disease.

\section{Conclusions}

Machine learning algorithms may be helpful to combine multimodal features that change over time and to adapt the probability for $\mathrm{DCl}$ occurrence to every single patient at different time points of the disease. 


\section{Distinguished Poster 21}

\section{Automated Extraction of Infarct Lesion and Net Water Uptake from Serial CT Images of Large Vessel Occlusion Stroke Patients}

Rajat Dhar, Atul Kumar, Yasheng Chen, Aaron Corbin, Ali Hamzehloo, Amin Abedini, Laura Heitsch, Jamal Derakhshan, Jin-Moo Lee

Neurology; Washington University in St. Louis School of Medicine, St. Louis, MO, United States

\section{Background \& Purpose}

Quantifying the extent of infarction and cerebral edema in patients with large-vessel occlusion (LVO) strokes is challenging. Lesional net water uptake (NWU) is a promising CT-based biomarker of evolving edema, but its measurement requires manually outlining infarcted tissue and mirrored regions in the contralateral hemisphere. We develop an imaging pipeline capable of automatically delineating the infarct region and calculating NWU from serial CTs of LVO patients.

\section{Methods}

We analyzed baseline and follow-up CTs from 26 patients with anterior circulation LVO. Infarct core was extracted using a CTP deconvolution algorithm and compared to RAPID volumes (present in 20 cases). Follow-up infarcts were segmented using a deep-learning algorithm trained on 265 manually-outlined infarcts. Baseline and follow-up core/infarct masks were flipped along a registered brain midline to generate mirror regions in the contralateral hemisphere. NWU was one minus the ratio of densities between these two regions, removing CSF and pixels outside thresholds of 20-80 (baseline/normal) and 0-40 (infarct). Automated results were compared with those using manually-drawn infarcts and an ASPECTS region-based method that samples densities within the core/infarct vs. normal hemisphere.

\section{Results}

Core masks extracted in 16 of 20 cases (median volume 28-ml, IQR 21-44) correlated with RAPID volumes $(r=0.87, p<0.0001)$. Baseline NWU using automated core was median $6 \%$ (IQR 3-8) and correlated with manual measurement $(r=0.69, p=0.002)$ and ASPECTS $(r=-0.74, p=0.0009)$. Thirty-two follow-up CTs were adequate quality and had visible infarcts without parenchymal hematoma: automated infarct volumes correlated to manually-drawn volumes ( $r=0.82)$. Fully automated NWU was median 24\% (IQR 19-27) and was highly correlated with NWU using manually-drawn infarcts ( $r=0.94)$ and the sampling-based method $(r=0.80$, both $p<0.0001)$. NWU increased over time and was higher in those with midline shift.

\section{Conclusions}

We developed an imaging pipeline capable of extracting metrics of evolving cerebral edema from serial CTs of stroke patients. This automated approach could be leveraged to study the impact of cerebral edema in large cohorts of patients. 


\section{Distinguished Poster 22}

\section{Klotho-VS Heterozygosity is Associated with Lower Risk of Lobar Intracerebral Hemorrhage}

JN Acosta, N. Szejko, CP Both, AC Leasure, D. Woo, CD Langefeld, A. Biffi, CD Anderson, J. Rosand, TM Gill, L. Sansing, KN Sheth, GJ Falcone

Department of Neurology, Yale University School of Medicine, New Haven, CT, United States

\section{Background \& Purpose}

Two missense variants in the Klotho gene $(\mathrm{KL})$ form the functional haplotype KL-VS. KL-VS-Het+ increases serum levels of Klotho and attenuates the risk of Alzheimer's Disease conferred by APOE epsilon 4. Our aims were to test the hypothesis that heterozygosity for KL-VS (KL-VS-Het+) lowers the risk of spontaneous intracerebral hemorrhage $(\mathrm{ICH})$ and test whether the association differs between lobar and non-lobar ICH or by APOE epsilon variants.

\section{Methods}

We conducted a genetic association study using individual-level data from European ancestry participants enrolled in 3 case-control studies of ICH. We used the genetic data to determine KL-VS heterozygosity status and APOE epsilon alleles. We tested for association between KL-VS-Het+ and ICH risk via study-specific logistic regression followed by fixed-effects, inverse-variance weighted metaanalysis using 12 to quantify heterogeneity. Given the known biological differences between lobar and non-lobar ICH, we conducted stratified analyses based on location. Additionally, we evaluated the role of KL-VS-Het+ in carriers of APOE epsilon 2 and 4 variants.

\section{Results}

A total of $1066 \mathrm{ICH}$ cases (464 lobar and 602 non-lobar) and 1073 controls were included in the study (mean age 69 [SD 14], female sex 919 [47\%]). KL-VS-Het+, present in 554 (26\%) participants, was associated with a lower risk of ICH (OR $0.81,95 \% \mathrm{Cl} 0.67-0.99 ; \mathrm{p}=0.04)$ without heterogeneity across studies $(12=0 \%)$. Stratified analyses indicated that KL-VS-Het+ was associated with a lower risk of lobar ICH (OR 0.68, 95\% Cl 0.52-0.88; $p=0.004$ ) but not of non-lobar ICH (OR 0.92, 95\% Cl 0.73-1.16; $p=0.48$ ), although the statistical interaction was not significant $(p=0.05)$. In secondary analyses, no significant interaction was found for the association between KL-VS-Het+ and APOE epsilon 2 or 4 carrier status.

\section{Conclusions}

KL-VS-Het+ is associated with a lower risk of $\mathrm{ICH}$. This protective association was stronger in lobar hemorrhages. Further research should evaluate these associations in non-Europeans and identify the mediating molecular pathways. 


\title{
Distinguished Poster 23
}

\section{Race and Ethnicity Influence Perihematomal Edema Volume in Supratentorial Intracerebral Hemorrhage}

\author{
JN Acosta, Y. Chen, CP Both, AC Leasure, F. Testai, D. Woo, J. Lee, KN Sheth, R. Dhar, GJ Falcone \\ Department of Neurology, Yale University School of Medicine, New Haven, CT, United States
}

\section{Background \& Purpose}

Perihematomal Edema (PHE) is a neuroimaging biomarker of secondary brain injury in patients with $\mathrm{ICH}$. There are limited data on racial/ethnic differences in the development of PHE. This dearth of data is partially driven by the time-consuming process of manually segmenting PHE. Our aim was to evaluate whether race and ethnicity influence baseline PHE volume in patients with spontaneous, non-traumatic intracerebral hemorrhage (ICH).

\section{Methods}

The Ethnic/Racial Variations in Intracerebral Hemorrhage (ERICH) study is a prospective, multicenter study of ICH that recruited 1,000 adult participants from each of three racial/ethnic groups (nonHispanic White, non-Hispanic Black, and Hispanic). We applied a previously validated deep learning algorithm to automatically determine PHE volumes on baseline CTs in these study participants. Quality control procedures were used to include only sufficiently accurate PHE measurements. Linear regression was used to identify factors associated with log-transformed PHE volume and to identify differences across Ethnic/Racial groups.

\section{Results}

Our imaging pipeline provided good quality baseline PHE measurements on 2,008 out of 3,000 ERICH study participants. After excluding infratentorial hemorrhages (273) and those with missing or null baseline ICH volume (49), 1,686 remained for analysis (median age 59 [IQR 51-71], 687 [41\%] female sex). Median PHE volume was 12.0 (IQR 4.8-27.1) for whites, 11.9 (IQR 4.5-26.1) for Hispanics, and 8.3 (IQR 3.0-19.2) for blacks. Compared to Blacks, Hispanics (beta $0.21 ; 95 \% \mathrm{Cl} 0.08-0.33 ; \mathrm{p}=0.001$ ) and Whites (beta $0.20 ; 95 \% \mathrm{Cl} 0.06-0.34 ; \mathrm{p}=0.005$ ) had higher baseline PHE volumes, in multivariable analysis adjusting for age, sex, time to $\mathrm{CT}$, ICH location, log-baseline ICH volume, log-baseline intraventricular volume, and systolic blood pressure on admission.

\section{Conclusions}

Race and ethnicity influence the volume of baseline PHE. Further studies are needed to validate our results and investigate the biological underpinnings of this difference. 


\section{Distinguished Poster 24}

\section{Comparison of Intermittent Versus Continuous Infusion Antihypertensives in Acute Ischemic Stroke}

Ashley Kamp, Wennie Huang, Timothy Lassiter, Shreyansh Shah, Beiyu Liu, Bridgette Kram

Duke University Hospital, Department of Pharmacy, Durham, NC, United States

\section{Background \& Purpose}

The optimal approach to blood pressure (BP) management in acute ischemic stroke remains unclear. The purpose of this study was to determine if an intermittent or continuous infusion antihypertensive strategy in patients with acute ischemic stroke facilitated timelier alteplase administration.

\section{Methods}

Patients $\geq 18$ years admitted to the emergency department (ED) between September 1, 2013 and August 31,2020 who received alteplase for acute ischemic stroke and required BP management with an intravenous antihypertensive were included in this multicenter, retrospective cohort study. Specific antihypertensives evaluated were intermittent labetalol and hydralazine and continuous infusion nicardipine and clevidipine. Exclusion criteria were initial administration of a non-study antihypertensive, initial study antihypertensive administration $>2$ hours prior to or any time following alteplase, administration of labetalol as a continuous infusion, or receipt of both an intermittent and continuous infusion antihypertensive prior to alteplase. The primary endpoint was the time from ED presentation to alteplase administration, which was evaluated using the Wilcoxon-Mann-Whitney test.

\section{Results}

A total of 179 patients were included. Of these patients, 122 received an intermittent antihypertensive and 57 received a continuous infusion antihypertensive. The median door-to-needle time was 53 minutes for patients who received an intermittent antihypertensive compared to 57 minutes for those who received a continuous infusion antihypertensive $(p=0.17)$. Secondarily, the proportion of patients who achieved the BP target $<185 / 110 \mathrm{mmHg}$ within 15 minutes of initial antihypertensive administration and the incidence of adverse events were similar between treatment groups. In cost analysis, intermittent antihypertensives were less expensive than continuous infusion antihypertensives (median cost: $\$ 2.20$ vs. $\$ 71.40$ ).

\section{Conclusions}

Among patients with acute ischemic stroke and uncontrolled BP, the initial use of an intermittent or continuous infusion antihypertensive did not significantly impact the time to alteplase administration. However, an intermittent antihypertensive strategy was associated with lower medication costs. 


\title{
Distinguished Poster 25
}

\section{Intravenous Formulation of Ganaxolone for the Treatment of Refractory Status Epilepticus: Safety Analysis of the Renal Function in a Phase 2 Open-Label, Dose-Ranging Study}

\author{
Maciej Gasior, Eva Rybak, Eugene Ramsay, Christa B. Swisher, Henrikas Vaitkevicius
}

Marinus Pharmaceuticals, Radnor, PA, United States

\section{Background \& Purpose}

IV ganaxolone, a neuroactive steroid that acts as a positive allosteric modulator of synaptic and extrasynaptic GABAA receptors, is an intravenous formulation solubilized by Captisol (betadex sulfobutyl ether sodium). Due to the potential for renal toxicity with Captisol at high doses, exposure was limited to 50 grams/day in a Phase 2 open-label study of IV ganaxolone in refractory status epilepticus (RSE). To assess renal safety, we monitored patients for acute renal injury (AKI) by following relevant laboratory parameters.

\section{Methods}

17 patients with RSE were enrolled in the study. Total duration of ganaxolone ranged from 48 to $96-\mathrm{h}$ followed by an 18-h taper. Serum creatinine ( $\mathrm{sCr}$ ), blood urea nitrogen (BUN), and estimated glomerular filtration rate (eGFR; calculated using Modification of Diet in Renal Disease) prior to ganaxolone, every 24 hours during infusion, and up to 4 weeks post-treatment were collected.

\section{Results}

Biomarkers of renal function were evaluated in 16 of 17 patients, excluding one patient entering the study on hemodialysis. At screening, mean (SD) $\mathrm{sCr}(\mathrm{mg} / \mathrm{dL}), \mathrm{BUN}(\mathrm{mg} / \mathrm{dL})$ and eGFR $(\mathrm{ml} / \mathrm{min} / 1.73 \mathrm{~m} 2)$ were 0.81 (0.29), 16.4 (8.2) and 102.5 (46.2), respectively. The mean maximum change from baseline up to 4 weeks post-ganaxolone were sCr -0.11 (0.1); BUN -3.9 (10.1); eGFR +24.5 (41.4). None of the patients exposed to ganaxolone had an increase in $\mathrm{s} C r \geq 0.3$ or 1.5 times baseline. No significant change in renal function $(p=0.24)$ during and following ganaxolone exposure was observed. Ganaxolone was generally well-tolerated, and no new safety findings emerged.

\section{Conclusions}

According to KDIGO guidelines, AKI is defined as an increase in $\mathrm{sCr}$ of $\geq 0.3 \mathrm{mg} / \mathrm{dL}$ within 48 hours or $\geq 1.5$ times baseline within 7 days. None of the patients in this study met this definition during or for four weeks after ganaxolone infusion. A Phase 3 study of IV ganaxolone in RSE is currently enrolling in the U.S. 


\section{Distinguished Poster 26}

\section{Focal Brain Cooling with Intranasal Cooler for Encephalopathy Prevention in Combat Casualties (ICEPICC) device For Prevention Of Secondary Brain Injury}

Ryan T. Myers, Neeraj Badjatia, Michael Richmond, Michaelina Dupnik, Nikole Shooshan, Amina Hasan Abedin, Andras Pungor, Kristian J. DiMatteo, Jonathan Morrison

Vivonics, Inc., Bedford, MA, United States

\section{Background \& Purpose}

Therapeutic hypothermia (TH) has been shown to reduce cerebral edema after traumatic brain injury; however, the systemic side effects of $\mathrm{TH}$ are a significant barrier to implementation. Herein we describe the initial application of an intranasal device that offers rapid onset of targeted, localized cooling in an experimental model.

\section{Methods}

We conducted a prospective two-arm study on 10 male swine to assess safety and efficacy of the ICEPICC device. Animals were randomized into two groups, the first received intranasal cooling, and a control had the ICEPICC device inserted, but not turned on. The swine were subjected to cerebral and cardiovascular instrumentation. Continuous data were collected throughout the study. Comparisons were made at baseline (BL), end-of-intervention (EOI), and end-of-study (EOS) between the groups, along with the CT perfusion (CTP) measurements.

\section{Results}

Baseline, cooling, and rewarming values of the cooled group were compared to the control group at the same time points. Intracranial temperature was compared between groups at the three time points and was found to be significantly different during cooling with an average temperature change of $+3.10 \mathrm{C}$ vs $+0.42 \mathrm{C}$ respectively $(p=0.008)$ and not significant during baseline and rewarming $(p=0.100$ and $p=0.421)$. Rectal temperatures were not significantly different at any point in the study between the two groups. Laser doppler perfusion and cerebral blood flow (CBF) were not significantly different between control and intervention groups.

\section{Conclusions}

Using the ICEPICC device to selectively cool the brain appears to be a safe and effective method for quickly achieving local temperature reduction. These findings provide safety and effectiveness data for proof of concept to pursue studies that will look to correct brain temperature in febrile patients within the clinic as well as at point of injury outside of the clinic for fast, focal, brain cooling. 


\title{
Distinguished Poster 27
}

\section{Preparing to Take Off the Training Wheels: Practice Management in Neurocritical Care}

\author{
Shweta Goswami, Eunice J. Lee, Rachael Scott, Pouya Ameli, Marc Babi
}

University of Florida - Neurology, Gainesville, FL, United States

\section{Background \& Purpose}

There has been a growing concern for the lack of practice management in medical education within the United States across many different specialties. Prior surveys have indicated a nearly universal lack of confidence in topics such as coding, reimbursement, resource allocation, ethics and malpractice. Simultaneously, primary care specialties such as Family Medicine have shown that formal practice management training is an effective approach to improving coding compliance, clinic productivity and patient outcomes. We sought to determine what strategies are being employed for practice management education in critical care, specifically neurocritical care.

\section{Methods}

To address this shortcoming within the neurocritical care specialty, the Neurocritical Care Society Trainee Section Leadership initiated a literature search in practice management education for critical care. We conducted a comprehensive literature search for keywords containing "practice management," "contract negotiation," or "ethics" within "(neuro)critical care" and "education."

\section{Results}

While there were studies looking at clinical neurocritical care education across multiple training levels and specialties (nursing vs. physicians), there are no published reports on practice management education in neurocritical care or critical care. Additionally, there are no reports examining the readiness for trainees to practice independently. To combat the lack of research on practice management and curricula, the Neurocritical Care Society Trainee Section set out to create a lecture series aimed at teaching specific topics within practice management, such as conflict resolution and billing and coding, and developed a pre- and post-survey to better quantify readiness of trainees to practice independently based on a developed curriculum.

\section{Conclusions}

As part of an effort to prepare Neurocritical care providers for independent practice, the Neurocritical Care Society Trainee Section was approved to host a series of lectures titled, "Preparing to Take Off the Training Wheels: Practice Management in Neurocritical Care." The goal is to bridge the knowledge gap in various topics within practice management and transition to independent practice. A series of guest speakers and panelists will be recruited to address common issues inherent to critical care practice but formally addressed within the current curricula, along with strategies to manage them. We plan to administer a voluntary pre- and post-survey to all attendees of the lecture series asking confidence in each topic of practice management to gauge effectiveness of this educational initiative on increasing practice management confidence in new practitioners. If anyone is interested in participating in the lecture series, please contact us at ncstraineesection@gmail.com 


\title{
Distinguished Poster 28
}

\section{Assessment of Cerebrovascular Autoregulation Before, During, and After Therapeutic Interventions Following Severe Traumatic Brain Injury: A Secondary Analysis of the BOOST-II Study}

\author{
Ayush Prasad, Jessica Kobsa, Alexandria Soto, Jennifer Kim, Emily J. Gilmore, Kevin Sheth, Charles \\ Matouk, Adam de Havenon, Ramon Diaz-Arrastia, Nils H. Petersen, BOOST-II Investigators \\ Yale School of Medicine - Division of Neurocritical Care and Emergency Neurology, New Haven, CT, \\ United States
}

\section{Background \& Purpose}

The BOOST-II randomized controlled trial used a tier-based management protocol based on brain tissue oxygen (PbtO2) and ICP monitoring to reduce brain tissue hypoxia (BTH) after severe TBI. To explore the underlying pathophysiologic mechanisms, we performed a secondary analysis assessing the relationship between BTH and blood pressure (BP). We hypothesized that BP management below the lower limit of autoregulation (LLA) leads to cerebral hypoperfusion and BTH, which can be improved with hemodynamic augmentation.

\section{Methods}

Of the 106 patients enrolled in BOOST-II, 55 patients had simultaneous recordings of arterial BP, ICP, and $\mathrm{PbtO} 2$. Autoregulatory function was measured by interrogating changes in $\mathrm{PbtO} 2$ in response to fluctuations in cerebral perfusion pressure (CPP) using time-correlation analysis. The resulting autoregulatory index (ORx) identified "optimal" CPP (CPPopt) and limits of autoregulation (LA) for each patient. Autoregulatory function and percent time of CPP outside personalized LA were calculated before, during, and after all interventions directed at optimizing CPP.

\section{Results}

Individualized LA were computed in 50 patients (age $38.2 \pm 18.2,76 \%$ male, monitoring time $92.8 \pm 42.4$ hours). We identified 35 episodes (in 6 patients) of BTH (PbtO2 $<20 \mathrm{mmHg}$ ) treated with CPP augmentation. Following each intervention, mean CPP increased from $73 \pm 14$ to $79 \pm 19 \mathrm{mmHg}(p=0.15)$, and mean PbtO2 improved from $18.4 \pm 5.6$ to $21.9 \pm 5.6(p=0.01)$, while autoregulatory function trended towards improvement (ORx: 0.42 vs. $0.37, p=0.14$ ). Though CPPopt and LA remained relatively unchanged, there was a significant decrease in the percent time of CPP below the LLA in the 60 minutes after compared to before an intervention ( $11 \%$ vs. $23 \%, p=0.05$ ).

\section{Conclusions}

Our analysis suggests that BTH is associated with cerebral hypoperfusion characterized by an increased time of CPP below the LLA. Interventions to increase CPP appear to restore autoregulation, resulting in improved $\mathrm{PbtO} 2$. Further studies to validate the importance of autoregulation as a modifiable variable, with the potential to improve outcomes, are needed. 


\title{
Distinguished Poster 29
}

\section{Quantification of Enlarged Perivascular Spaces Detected on MRI is Predictive of 6 Month Outcome Following Traumatic Brain Injury}

\author{
Krupa Savalia, Jason Yoon, Alexa Walter, Ramon Diaz-Arrastia, Danielle Sandsmark \\ Department of Neurology, University of Pennsylvania, Philadelphia, PA, United States
}

\section{Background \& Purpose}

Perivascular spaces (PVS) are fluid-filled regions surrounding brain arterioles, venules, and capillaries important in brain metabolite clearance, and could contribute to neuroinflammatory and cerebrovascular disease. Enlarged PVS (ePVS), detected on conventional brain MRI, are a marker of PVS and microvasculature dysfunction. Given that microvascular abnormalities play an important role in traumatic brain injury (TBI), we examined the prevalence of MRI-detected ePVS in individuals with TBI and their relation to clinical outcomes.

\section{Methods}

Twenty-five patients with non-penetrating TBI (mean[SD] age: 36.1[13.8] years, 80\% male, median GCS:15(IQR: 14-15)) were enrolled within 24 hours of admission to a Level 1 Trauma Center. Participants underwent a research $3 T \mathrm{MRI}$ and outcome measures were collected at 2 weeks ( $15.5 \pm 3.86$ days) and 6 months (191.4 \pm 13.38 days) post-injury. ePVS burden was quantified using T1 and FLAIR sequences. Hypointense ePVS were counted from representative slices in the centrum semiovale, basal ganglia, and midbrain. A board-certified neurologist (JY) was the primary rater with a second neurologist (KS) used to determine interrater reliability. Data were analyzed both as continuous (total number of hypointensities) or by category ( $\leq 10,11-20,21-40$, and $>40$ hypointensities).

\section{Results}

Interrater reliability was calculated (ICC $=0.744$ for continuous data; kappa=0.349 for categorical data). There was no significant change in ePVS from 2 weeks $(21.94 \pm 9.87)$ to 6 months $(19.62 \pm 8.05$; $t=1.163$, $\mathrm{p}=0.256$ ). Pearson correlations showed a significant relationship between 6 month GOS-E and 2 week ePVS category ( $r=0.439, p=0.020)$. Linear regression showed that 2 week ePVS category predicted GOS-E at 6 months $(F(1,22)=5.25, p<0.05 ; R 2=0.16)$.

\section{Conclusions}

ePVS burden in the acute phase following injury may predict functional outcome. Our data reveal that ePVS burden does not change significantly over the 6 months following mild TBI. Further investigation may help elucidate how ePVS can serve as a marker to understand the natural progression and pathophysiology of TBI. 


\title{
Distinguished Poster 30
}

\section{Development and Validation of an Open-Source Optimum Cerebral Perfusion Pressure Tool to Guide Precision Care in Severe Traumatic Brain Injury}

\author{
Brandon Foreman, Fei Li, Eric S. Rosenthal, Craig Maddux, Daniel Habboush, Zack Goldblum, Dick \\ Moberg
}

University of Cincinnati, Department of Neurology \& Rehabilitation Medicine, Cincinnati, OH, United States

\section{Background \& Purpose}

Current guidelines recommend empiric targets for cerebral perfusion pressure (CPP) $60-70 \mathrm{mmHg}$ after severe traumatic brain injury (sTBI). However, autoregulatory dysfunction is common and tailoring care toward an optimum CPP (CPPopt) holds promise to improve precision of care. Current analytics are limited in integration with commercial Electronic Health Record (EHR) systems necessitating development of valid, open-source solutions.

\section{Methods}

A prototype CPPopt graphical user interface (CPPoptGUI) was developed to harness second-to-second data to calculate and display a CPPopt. The reference standard CPPopt was calculated using ICM+ (University of Cambridge Enterprise; Cambridge, UK) in a retrospective cohort of STBI patients from a level I trauma center. Agreement was assessed using a mixed effects model adjusting for repeated measures, between-subject and physiologic variability.

\section{Results}

A validation cohort of $n=10$ sTBI patients (average age $34.3+/-14.8$ years; $8 / 10$ male) with 31,352 minutes of monitoring data was used. ICM+ yielded 26062/28725 (90.7\%) CPPopt measurements; CPPoptGUI yielded 24680/28725 (85.9\%). Average (+/-standard deviation) values of CPPopt were similar (ICM+80.1+/-13.8 vs CPPoptGUI 79.8+/-13.1 mmHg). There was strong correlation $(r=0.77 ; p<0.001)$ even when stratifying for decompressive hemicraniectomy. The differential bias was $13.08 \mathrm{mmHg}$ [95\% Cl 11.70-14.47), but variance between ICM+ and CPPoptGUI was lower than that associated with between-subjects, between-measurements, and within-CPP differences. The mean $\triangle$ CPP (CPP-CPPopt) was $-4.0+/-11.5(I C M+)$ vs $-3.7+/-10.8$ (CPPoptGUI); both were significantly associated with mortality (ttest; $p<0.001)$.

\section{Conclusions}

We created and validated a prototype application for CPPopt and used robust repeated measures models to demonstrate reasonable accuracy and showed that both methods yielded similar average values and strongly correlated with each other and with mortality. Through the use of open-source tools for real-time conversion and integration with the EHR, we envision moving this analytic tool into the ICU to enhance precision care. 


\section{Distinguished Poster 31}

\section{Improving Access to Hospice for Comfort-Focused Care Patients in the Neurocritical Care Unit}

Winnie Lau, Jeffrey Soltes, Jared Lowe, Margret Drickamer, Casey Olm Shipman

University of North Carolina School of Medicine, Department of Neurology, Chapel Hill, NC, United States

\section{Background \& Purpose}

When families opt to pursue comfort-measures only (CMO) and compassionate extubation for neurocritically ill patients, barriers to hospice can be significant for intubated neurologic patients admitted to hospitals without onsite inpatient hospice facilities. This is due to logistical challenges of transfer to offsite hospice, as well as the inherent limitations in predicting survival time after extubation. Utilizing a quality improvement approach, we implemented a standard pathway to facilitate efficient offsite inpatient hospice transfers for mechanically ventilated NSICU patients transitioning to CMO.

\section{Methods}

Case reviews of all patient mortalities were performed by two neurointensivists, case management and a quality improvement project manager. Root cause analyses were conducted to identify barriers to hospice transfers. An educational campaign was developed with contributions from palliative care services and case management on hospice eligibility and transfer processes. Data-driven audit feedback strategies were employed to reinforce provider engagement and process adherence. Primary outcome was hospice transfer rate (numerator = \# of patients transferred to hospice; denominator = \# of patients transferred to hospice combined with \# of patients who died inpatient and were cared for in the NSICU at any point during hospital admission).

\section{Results}

Between January 2020 and April 2021, a total of 66 patients were transferred from the NSICU to inpatient hospice. Approximately half of patients were intubated at the time of transfer. Median hospice transfer rate from NSICU for eligible patients was $50 \%$ during the intervention period. Average hospice transfers per month increased from 0.5 pre-intervention to 3.87 post-intervention, without concomitant increase in overall mortality.

\section{Conclusions}

Transfer of mechanically ventilated patients to inpatient hospice is feasible and requires collaboration with local hospice facilities, primary intensive care teams, and case management. This intervention significantly improved access to hospice care for eligible patients without increasing overall mortality. Future research is needed to evaluate costs and patient-family experience. 


\section{Distinguished Poster 32}

\section{Different Cardiac Arrest Etiologies Lead to Differences in Peripheral Perfusion and Neurological Outcome Post-Resuscitation}

Sangwoo Han, Afsheen Bazrafkan, Masih Rafi, Niki Maki, Shirin Mansoori Dara, Alyssa Avilez, Ashwin Nath, Haein Yun, Kieran S. McCluskey, Rounak Baghbaninogourani, Stuti Agrawal, Robert H. Wilson, and Yama Akbari

University of California, Irvine. Department of Neurology, Irvine, CA, United States

\section{Background \& Purpose}

Cardiac arrest (CA) survivors often have neurological deficits that significantly impact their daily lives. Changes in peripheral blood pressure (BP) during the highly dynamic first 10 min post-resuscitation may inform early hemodynamic interventions to improve recovery.

\section{Methods}

In 48 male Wistar rats, femoral BP was measured during CA and cardiopulmonary resuscitation (CPR). 31 rats underwent asphyxial CA leading to pulseless electrical activity (PEA), while 17 were given intravenous $\mathrm{KCL}$ injection causing near-immediate asystole. These two groups mimicked different types of non-shockable CA found in patients. We quantified features of the BP curves during the hyperemic phase shortly post-CPR. We measured time to reach hyperemic peak, slope of BP curve between end of CPR and hyperemic peak, and absolute value of BP at hyperemic peak. Neurological Deficit Score (NDS) was used as an outcome measure at $4 \mathrm{hr}, 24 \mathrm{hr}, 48 \mathrm{hr}$, and $72 \mathrm{hr}$ post-CPR.

\section{Results}

At $24 \mathrm{hr}$ post-CPR, asystole CA rats had higher NDS $(\mathrm{p}<0.05)$ than PEA CA rats, but by $48 \mathrm{hr}$ post-CPR, the two groups both maximized their NDS score. Interestingly, asystole rats had a shallower BP slope from end of CPR to hyperemic peak ( $p<0.0001)$ and lower BP at hyperemic peak $(p<0.0005)$. In PEA rats, higher hyperemic BP peak was associated with worse 24hr NDS $(p<0.005, r=-0.53)$, but in asystole rats, higher hyperemic peak correlated with better $24 \mathrm{hr}$ NDS. Asphyxia and asystole rats had similar time to the hyperemic peak.

\section{Conclusions}

In our preclinical model, we observe that $\mathrm{KCL}$-induced asystolic CA results in better post-CPR outcome than asphyxia-induced PEA CA. There may be different optimal BP targets for different types of CA, with too high of perfusion in asphyxial CA rats being worse, and too low of perfusion in KCL rats being worse. Precision-targeted BP to target optimal ranges for different types of CA may help to optimize neurological recovery post-CA. 


\section{Poster 33}

\section{A Case of CADASIL with Simultaneous Bilateral Intracerebral Hemorrhages}

Hitoshi Kobata; Akihiro Kambara, Takayuki Morimoto

Osaka Mishima Emergency Critical Care Center, Takatsuki, Japan

\section{Background \& Purpose}

CADASIL (cerebral autosomal dominant arteriopathy with subcortical infarcts and leukoencephalopathy) is a hereditary cerebral small vessel disease caused by a mutation in the NOTCH3 gene. The typical clinical manifestation is ischemic events, dementia, and migraine with aura. Although asymptomatic microbleeds are frequently detected in MRI, symptomatic intracerebral hemorrhage (ICH) is extremely rare. To the best of our knowledge, there are no reports of CADASIL with simultaneous bilateral ICH.

\section{Methods}

Case report and review of the literature.

\section{Results}

A 52-year-old woman, who had been diagnosed as CADASIL (NOTCH3 exon19 Cys1015Arg mutation), was found in a coma and transferred to our hospital. She had a history of cerebral infarction two years ago and was taking antiplatelet medication. She also had a history of migraines. On arrival, her Glasgow Coma Scale score was 5 (E1V1M3), and the blood pressure was 190/111 mmHg. Head CT scans showed hemorrhages in the left caudate nucleus to the putamen and the right corona radiata to the globus pallidus with intraventricular hemorrhage. Head T2*-weighted MRI showed a increased number of microbleeds compared to 2 years ago. She underwent medical treatment, including ventilatory support and blood pressure control and was transferred to the hospital in an unconscious state.

\section{Conclusions}

Vulnerability of blood vessels and disruption of the cerebral blood barrier are recognized to occur in CADASIL. A recent study reported the prevalence of cerebral infarction and ICH in CADASIL are significantly higher in patients with hypertension, as is the number of cerebral microbleeds. Moreover, a meta-analysis suggests that the risk of $\mathrm{ICH}$ is more significant in females with migraines. Therefore, strict blood pressure control, prophylactic treatment of migraine, and prudent follow-up MRI are warranted to reduce the $\mathrm{ICH}$ risk in caring for CADASIL patients. 


\section{Poster 34}

\section{A Case of Lemierre's Disease, Cerebral Venous Sinus Thrombosis, Streptococcus intermedius Meningitis due to Acute Mastoiditis after Persistent Acute Otitis Media}

Essie P. Mathews, Thuy-Tien Ho, Jia Romito

UT Southwestern Medical Center, Neurocritical Care, Dallas, TX, United States

\section{Background \& Purpose}

This case details a 20-year-old male who presents with Lemierre's disease, cerebral venous sinus thrombosis, elevated ICP, Streptococcus intermedius meningitis in the setting of acute mastoiditis due to persistent acute otitis media, and incidentally positive COVID-19 PCR. Early recognition and treatment led to the reversal of rapid neurological deterioration with good neurological outcome.

\section{Methods}

Electronic medical record chart review and PubMed.

\section{Results}

A 20-year-old Hispanic male, with a history of marijuana abuse and recent persistent acute otitis media despite treatment with amoxicillin/clavulanic acid, presented with decreasing level of consciousness, left hemiparesis progression to hemiplegia, anarthria, emesis, and fever $>39^{\circ} \mathrm{C}$. Incidentally, he was found to be COVID-19 PCR positive, without respiratory distress while on room air. One hour prior to his neurological deterioration, his parents reported he was at his baseline without any deficits. CT head was concerning for right mastoiditis with septic thrombophlebitis; extensive venous sinus thrombosis involving the superior sagittal sinus extending through the right transverse sinus to the level of the right sigmoid sinus and jugular bulb. Lumbar puncture was notable for a closing pressure of $39 \mathrm{mmHg}$. Meropenem dosed for CNS coverage and heparin infusion were initiated. Hyperosmotic therapy with administration of both mannitol and hypertonic saline reversed his progressive stupor, and he regained the ability to state his name. He underwent mastoidectomy, tympanostomy tube placement, and craniotomy with evacuation of empyema that grew Streptococcus intermedius. By hospital discharge, he had neurologically improved to only having a mild left facial droop with mRS 0 .

\section{Conclusions}

This is a rare case of an otogenic source of Lemierre's disease with cerebral venous sinus thrombosis, Streptococcus intermedius meningitis, and COVID-19 with an initial presentation of rapid neurological deterioration that received timely implementation of antimicrobial, anticoagulation, elevated ICP management, and surgical therapies, resulting in good neurological outcome. 


\section{Poster 35}

\section{A Case of New Onset Status Epilepticus in an Adult, Secondary to Linear Scleroderma En Coup De Sabre}

Hera Kamdar; Jan Bittar, Sarita Maturu

The Ohio State University, Columbus, $\mathrm{OH}$, United States

\section{Background \& Purpose}

En coup de sabre (ECDS) is a rare form of linear scleroderma that is more predominant in females and children, with approximately $67 \%$ of patients diagnosed prior to the age of 18 . This connective tissue disorder is characterized by thickening of the frontoparietal area of the head that forms a depressed groove on the scalp and eventually develops linear alopecia. The neurological manifestations of ECDS are typically recognized after the skin presentation with complex partial seizures being the most frequently reported neurological abnormality. Here we present a unique case of ECDS diagnosed in adulthood, after presentation of new onset status epilepticus, and before the diagnosis of skin disease.

\section{Methods}

Retrospective chart review of a single case with obtained consent.

\section{Results}

A 58-year-old female with no prior history of seizures or associated risk factors of epilepsy was admitted to the Neurocritical Care Unit for management of new onset status epilepticus. Infectious, toxic and metabolic workup were unremarkable. Brain magnetic resonance imaging (MRI) revealed right cerebral hemispheric and cortical fluid-attenuated inversion recovery (FLAIR) hyperintensity and edema mainly involving the right frontal lobe. There was also associated dural enhancement in this region. Continuous electroencephalogram demonstrated 20 clinical and subclinical seizures arising from the right frontocentral region, consistent with focal status epilepticus. Careful skin examination revealed an atrophic scar on her right scalp overlaying the cortical abnormalities seen on MRI. This scar was reportedly present since birth. Further workup with skin biopsy and rheumatologic serum studies, including AntiScleroderma Antibody, were nonspecific. However, given her clinical presentation and dermatologic examination, she was diagnosed with ECDS. The patient improved after anti-epileptic medication titration and was discharged with planned immunosuppression therapy for her diagnosis of ECDS.

\section{Conclusions}

Our case highlights the importance of careful skin examination in patients who present with unexplained late-onset status epilepticus. 


\title{
Poster 36
}

\section{A Case Report Of Severe Foodborne Botulism}

\author{
T. Burton, B. Boling, B. Dahl, A. Malone, J. Huston, K. Sproul
}

University Of Kentucky, Lexington, KY, United States

\section{Background \& Purpose}

Botulism is a paralytic syndrome characterized by progressive symmetric muscle weakness leading to paralysis and even death. It is caused by the release of neurotoxin by Clostridium Botulinum. Foodborne botulism is extremely rare, with $<25$ cases reported annually in the US. Overall mortality rate is between 5\%-10\% making prompt diagnosis and treatment with antitoxin essential. Because of its rarity, misdiagnosis is common leading to delayed treatment and ultimately poorer outcomes.

\section{Methods}

This is a case report using a retrospective chart review. An in-depth review of the literature was conducted on botulism, its presentation, diagnosis, treatment, and outcomes.

\section{Results}

A 73-year-old male presented to the Emergency Department with nausea, dizziness, diplopia, and left eye ptosis rapidly progressing to bilateral ptosis, sluggish pupils, and bulbar weakness with difficulty in breathing. He was intubated and transferred to the ICU for care. Over the next 48 hours he developed bilateral mydriasis and progressive descending weakness until he reached total paralysis. Upon further questioning, his wife reported exposure to potentially tainted shellfish, prompting the concern for botulism which was confirmed by laboratory testing. Antitoxin was administered on day four after arrival from the CDC; however, no improvement was seen following antitoxin administration . Over the next 35 days he suffered numerous complications related to paralysis and prolonged mechanical ventilation. After developing sepsis, his family elected to withdraw life support and he died shortly thereafter.

\section{Conclusions}

Botulism symptoms can often be complicated, and presentation may mimic a number of neurological disorders. Treatment with botulism antitoxin is time sensitive and delayed diagnosis may lead to increased mortality and morbidity. Despite its rarity, botulism should be on the differential list for patients presenting with symmetrical progressive weakness. 


\section{Poster 37}

\section{A Curious Case of COVID-19 - Seizures and Hypoxic Ischemic Encephalopathy in an Infant}

Conall Francoeur, Marianne Dufour, Marie-Ève Samson, Renée-Myriam Boucher, Roseline Thibeault,

CHU de Québec - Université Laval, Department of Pediatrics, Quebec City, Quebec, Canada

\section{Background \& Purpose}

SARS-CoV-2 has primarily been associated with respiratory disease. Neurologic involvement however has been reported in a fifth of children with COVID-19 related admission, both with primary COVID-19 disease and in association with multisystem inflammatory syndrome (MIS-C) (1). Involvement is usually transient but some patients develop life-threatening neurologic conditions $(1,2)$. Neonates tend to be asymptomatic or have a mild clinical course (3) but little known about COVID-19 infection and neurologic manifestations in infants.

\section{Methods}

We present the case of a COVID-19-positive 4 day-old who presented with seizures and whose course was complicated by hypoxic ischemic encephalopathy (HIE).

\section{Results}

A 4-day-old term male with an unremarkable perinatal history presented with recurrent seizures. The seizures were tonic-clonic with right-sided predominance and lasted 30-120 seconds and were associated with desaturations. The clinical seizures stopped with lorazepam and phenobarbital. CT showed no acute abnormalities. EEG showed a post-ictal pattern with bilateral dysrhythmia and bilateral epileptic foyers. Investigations revealed a positive COVID-19 nasopharyngeal PCR but normal electrolytes and a normal metabolic work-up. Chest x-ray and lumbar puncture were unremarkable, including CSF with a negative COVID-19 PCR. The neurologic exam improved with the exception of mild hypotonia but after 12 hours, the patient became more lethargic with a Glasgow Coma Scale that dropped from 15 to 10-11. He developed occasional respiratory pauses and desaturations that responded high-flow oxygen therapy. MRI was consistent with HIE with zones of diffusion restriction in the white matter bilaterally. After 48 hours, the baby became more reactive but remained hypotonic. At 5-month follow-up, he continued to exhibit gross motor delay with axial hypotonia and an MRI that shows cerebral atrophy.

\section{Conclusions}

COVID-19 infection in infants and children has generally been associated with mild disease. Clinicians however should recognize the potential for more severe presentations including neurologic manifestations such as seizures. 


\title{
Poster 38
}

\section{A Novel Mutation in a Late-onset Multiple acyl-CoA Dehydrogenase Deficiency Identified with Metabolic Crisis as a Result of Fasting Conditions}

\author{
Soo-Hyun Park, Tae Jung Kim, Sang-Bae Ko
}

Inha University Hospital, Department of Neurology and Critical Care Medicine, Incheon, Republic of Korea

\begin{abstract}
Background \& Purpose
Multiple acyl-CoA dehydrogenase deficiency (MADD), caused by mutations in the electron transfer flavoprotein (ETF), disrupts mitochondrial $\beta$-oxidation metabolism with energy production. MADD is variable in severity, ranging from nausea to fatal symptoms according to age of onset with various biochemical abnormalities. It is rare for late-onset presenting with metabolic crisis to exhibit a sudden mental status change with seizure. Herein, we present an interesting case of a late-onset MADD unreported gene mutation.
\end{abstract}

\section{Methods}

MADD is known to occur to mutations in the electron transfer flavoprotein dehydrogenase (ETFDH) gene. Exome sequencing and elevated plasma acylcarnitine levels can be useful in identifying genomic alterations for targeted therapy.

\section{Results}

We report the case of a 17-year-old woman. She had been presenting with progressive proximal muscle weakness, swallowing and spitting difficulty. Because these progressive symptoms aggravated, she was hospitalized. After the patient suffered from seizure with a sudden change in mental status, which led to rhabdomyolysis (CK $6049 \mathrm{IU} / \mathrm{L})$, hyperammonemia $(327 \mu \mathrm{mol} / \mathrm{L})$, hypoglycemia $(5 \mathrm{mg} / \mathrm{dL})$, respiratory failure with acidosis ( $\mathrm{pH}: 7.28, \mathrm{pCO} 2: 28 \mathrm{mmHg}, \mathrm{HCO}-: 14 \mathrm{mmol} / \mathrm{L}$ ), and arrhythmia (138 beats/min) after starving for several hours. We suspected a fatty acid oxidation disorder due to prolonged fasting and elevated plasma acylcarnitine, and genetic testing identified a novel homozygous mutation in the ETFDH, c.1286G>A (p.G429E). After continuous renal replacement therapy due to worsening rhabdomyolysis (CK $12781 \mathrm{IU} / \mathrm{L}$ ) and nutritional support with a high complex carbohydrate, low fat, and protein formula, the patient recovered.

\section{Conclusions}

Patients who experience altered mentality with seizure, hyperammonemia, arrhythmia, and acidosis with acute respiratory failure after starving should undergo laboratory tests to detect metabolic crisis. If laboratory test results represent rhabdomyolysis, hypoglycemia, hyperammonemia, clinicians should suspect a heredity metabolism deficiency, MADD. Rapid and prompt treatment are important because of acute complications. Our report help understands patients' characteristics with MADD and presents it as a novel mutation. 


\title{
Poster 39
}

\section{A Rare Cause of Meningoencephalitis - Balamuthia mandrillaris}

\author{
Varun Shah, Anna Finley Caulfield
}

Division of Neurocritical Care, Department of Neurology, Stanford University, Palo Alto, CA, United States

\section{Background \& Purpose}

Balamuthia mandrillaris is an amoeba that rarely causes granulomatous meningoencephalitis. Two hundred cases have been reported since first identified in 1990, with one case annually in the United States.

\section{Methods}

Clinical observation, data review, and autopsy.

\section{Results}

A 76-year-old man who 10 years prior survived laryngeal squamous cell carcinoma with laryngectomy, tracheostomy, and G-tube placement, presented with fevers and failure-to-thrive. For the previous 8 months, he had worsening behavioral changes and aggression, resulting in incarceration. Following admission, he became agitated and altered. Serial CSF analyses revealed lymphocytic then neutrophilic predominant pleocytosis and hypoglycorrhachia. Extensive studies for bacterial, fungal, and viral organisms were negative. Despite empiric broad antimicrobial, TB and fungal coverage, he became comatose. Serial head CT revealed worsening hydrocephalus despite external ventricular drains. Head MRI showed acute infarcts (left posterior internal capsule and putamen, left ventral pons, right insular cortex), extensive restricted diffusion and associated edema of the meningeal surfaces and cortices, as well as periventricular regions, consistent with extensive meningitis/ventriculitis, and without nodular cortical enhancement at the skull base often characteristic of TB or fungal infection. With loss of many brainstem reflexes and motor responses, family withdrew care. He died 8 days after admission. Brain autopsy identified granulomatous amebic meningoencephalitis from Balamuthia mandrillaris.

\section{Conclusions}

Balamuthia mandrillaris is a rare cause of meningoencephalitis with a high case-fatality rate $(>90 \%)$. It has been found in soil, dust, and fresh water in warmer climates of South America and southwestern United States. Infections occur in immunocompetent and immunocompromised individuals through direct inhalation or skin break contamination, which, in case reports, most often is related to soil exposure. CNS infection results in subacute to chronic progressive neurological symptoms. Brain imaging has reported multiple, ring-enhancing cystic lesions, hydrocephalus, and infarctions related to small vessel angiitis. Limited diagnostic tests include indirect-immunofluorescence and PCR. CDC should be contacted for suspected cases. 


\title{
Poster 40
}

\section{A Rare Reversible ICU Non Compressive Encephalomyelopathy Where Basic Physical Examination Undeniably the Gold Standard}

\author{
Samia Qadir, Stephen Tulley, Raju Z. Abraham
}

Riverside Medical Center - Internal Medicine, Kankakee, IL, United States

\section{Background \& Purpose}

Subacute combined degeneration of the spinal cord (SCD) is a neurodegenerative disease caused by cobalamin deficiency and characterized by demyelination of spinal dorsal and lateral columns. Symptoms include sensory deficits, ataxia, spasticity and cognitive dysfunction. Classical SCD presents with macrocytic anemia, increased methylmalonic acid (MMA) and homocysteine (HC) levels with demyelination noted on spinal magnetic resonance imaging (MRI). This case highlights clinical diagnosis of SCD and response to treatment of the neurological symptoms before and after cobalamin administration without classical laboratory or MRI abnormalities.

\section{Methods}

Neurological response, MMA and $\mathrm{HC}$ levels were measured before and after cobalamin.

\section{Results}

62-year-old female was admitted for acute encephalopathy, and severe calorie malnutrition. She had been experiencing unsteady gait with recurrent falls and decreased appetite. Patient was intubated and monitored on minimal sedation. MRI Brain, electroencephalogram (EEG) and lumbar puncture were unremarkable. Neurological exam showed bilateral lower extremity hyperreflexia with patellar and ankle clonus and upgoing plantars. New onset macrocytic anemia was noted. Normal cobalamin levels with high normal serum MMA and normal HC levels were noted (Fig 1). Spinal MRI was unremarkable. Given non compressive myelopathy and a high index of suspicion, patient was empirically treated with cobalamin. Patient recovered normal mentation, was extubated and was noted to have impaired proprioception and pallesthesia. MMA levels decreased, and symptoms of clonus, hyperreflexia and pallesthesia resolved.

\section{Conclusions}

SCD can present with a varying picture. Diagnosis is delayed because cobalamin levels are normal with no anemia or elevated mean corpuscular volume. Patients with neuropsychiatric symptoms of unclear etiology should be tested for cobalamin deficiency. Detection of lateral and dorsal column involvement in a non compressive myelopathy can be a crucial clue to warrant therapeutic trial. Monitoring neurological signs and MMA levels before and after cobalamin administration can further validate diagnosis of SCD. 


\title{
Poster 41
}

\section{Acute Necrotizing Encephalopathy due to SARS-CoV-2 in a Pregnant Female}

Hannah Breitl, Yazan Radaideh, Jean Slana, Lauren Koffman, Sayona John

\author{
Rush University Medical Center, Section of Neurocritical Care, Chicago, IL, United States
}

\section{Background \& Purpose}

We report a rare case of acute necrotizing encephalopathy (ANE) due to SARS CoV-2 in a pregnant female presenting with bilateral thalamic lesions. ANE is typically a parainfectious phenomenon most commonly described in children and associated with viral infections such as influenza-A. Our case highlights the need for consideration of this rare clinical entity in association with SARS CoV-2.

\section{Methods}

Patient was admitted to the neurosciences ICU and underwent extensive clinical workup. Literature review was conducted on ANE, neurologic manifestations of SARS CoV-2, and manifestations of SARS CoV-2 in pregnancy.

\section{Results}

19-year-old female at 33-weeks gestation with limited prenatal care presented with one day of confusion after a one-week prodrome of chest pain, tachypnea, dyspnea, nausea and vomiting. She tested positive for SARS CoV-2. CT brain revealed bilateral thalamic hypodensities, initially concerning for a cerebrovascular event. She was awake but globally aphasic with right homonymous hemianopsia, left gaze preference, and right upper extremity weakness. She was intubated for acute respiratory distress, then requiring emergent bedside Cesarean-section due to worsening fetal status. MRI revealed T2-FLAIR hyperintensities in bilateral thalami and caudate nuclei with hemorrhage and diffusion restriction, and T2-FLAIR hyperintensities in bilateral hippocampi, right parietal deep white matter, and bilateral posterior frontal white matter consistent with ANE, presumably due to SARS-CoV-2. Treatment with corticosteroids resulted in significant clinical improvement. On discharge patient was apathetic but otherwise neurologically intact. She was discharged to acute rehabilitation. The healthy neonate was discharged home.

\section{Conclusions}

This is the fourth reported case of ANE associated with SARS CoV-2 and first reported case of ANE in pregnancy. In SARS CoV-2, ANE should be considered in the differential for bilateral thalamic lesions as early immunotherapy can improve outcomes. While such lesions often portend a poor prognosis, our patient's excellent recovery suggests that outcomes depend on etiology of the lesions. 


\section{Poster 42}

\section{Acute Onset Painful Ophthalmoplegia: Considerations and Care Management}

Karina Burda, Tzu-Ying Chuang, Ephrem Teklemariam, M. Kamran Athar

Thomas Jefferson University Hospital Department of Neurology, Philadelphia, PA, United States

\section{Background \& Purpose}

Painful ophthalmoplegia is associated with a variety of processes localizing to the cavernous sinus, with frequent focus on cavernous sinus thrombosis as an etiology. However, consideration should be given to an entity associated with granulomatous inflammation of the cavernous sinus known as Tolosa-Hunt syndrome (THS), a rare diagnosis of exclusion which is remarkably steroid-responsive. We present a case of THS which highlights the importance of its consideration.

\section{Methods}

N/A

\section{Results}

A 45-year-old previously healthy man presented seven days after COVID-19 vaccination with a three-day history of severe left-sided headache, left eye pain with progressive left eye ptosis, decreased vision, and binocular diplopia. His examination was notable for left eye ptosis with an afferent pupillary defect and complete ophthalmoplegia. Labwork was unremarkable with exception of mild hyponatremia and elevated C-reactive protein. Head CT demonstrated hyperattenuation of the left cavernous sinus. Contrast-enhanced MRI of the brain and orbits as well as MRA/MRV of the head demonstrated bilateral perineural enhancement surrounding bilateral optic nerve sheaths and ill-defined enhancement in the left orbital apex extending into the cavernous sinus. Possible superimposed thrombosis was suggested but later deemed not definitively present. He was initially treated with broad-spectrum antibiotics, which were stopped after infectious studies (including cerebrospinal fluid analysis) showed no evidence of infection. His imaging findings were discussed with neuroradiology and thought to likely represent an underlying inflammatory process. Given suspicion for Tolosa-Hunt syndrome, empiric intravenous methylprednisolone was initiated. He had significant improvement in pain with minimal improvement in ptosis and eye motility. He was ultimately discharged home on an oral steroid regimen.

\section{Conclusions}

This case highlights the importance of considering common presentations of an uncommon disease in evaluation of painful ophthalmoplegia, as this can minimize unnecessary exposure to antibiotics and anticoagulants. Furthermore, early recognition of THS can lead to early symptomatic relief given its steroid-responsive nature. 


\section{Poster 43}

\section{Acute Quadriplegia as the Presenting Symptom of Sjögren's Acute Cervical Myelitis}

Anna Finley Caulfield, Phuong Thi Hoang, Nick Murray, Spencer Craven, Kyle Lyman, Brian J. Scott

Stanford University Department of Neurology and Neurological Sciences, Palo Alto, CA, United States

\section{Background \& Purpose}

Sjögren's syndrome is a well-described immune mediated condition with peripheral and, less commonly, central nervous system (CNS) manifestations; however, CNS manifestations are rarely the presenting symptom.

\section{Methods}

Direct clinical observation and management as well as data review of the electronic medical record.

\section{Results}

A 25-year-old previously healthy woman presented with headache and acute quadriparesis (initially arms weaker than legs). Within 24 hours, she was intubated for acute respiratory failure. Her examination revealed normal cranial nerves, flaccid quadriplegia, preserved proprioception, decreased sensation to pinprick (arms worse than legs), areflexia, urinary retention, and dysautonomia. Her cervical spine MRI demonstrated a non-enhancing, infiltrative, and mildly expansile T2/STIR hyperintensity within the right more than left anterior gray matter of the cord extending from C2 to C7; also, subsequently involving bilateral lateral cord white matter and anterior white matter at a few levels. Head and neck CTA, cervical spine dynamic MRA, and successive CSF tests (including infectious and autoimmune testing) were normal. Serum autoimmune tests returned negative for NMO/Aquaporin4IgG FACS and MOG FACS and positive for Anti-SSA and SSB antibodies. Lower lip minor salivary gland biopsy demonstrated two foci of lymphoid aggregates enumerated at greater than 50 mononuclear cells adjacent to normal-appearing mucous acini, consistent with a diagnosis of Sjögren's syndrome.

\section{Conclusions}

Sjögren's syndrome uncommonly manifests in the central nervous system as transverse myelitis. In case series, $15 \%$ of patients have cervical involvement and often longitudinally extensive ( $>3$ vertebrae) involvement. While there is an association between Sjögren's syndrome and neuromyelitis optica spectrum disorder, we did not find this in our patient. She was empirically treated with five days of methylprednisolone and IVIg with some improvement in motor strength. After the biopsy results, she was prescribed daily prednisone and subsequently consented to cyclophosphamide. Seven weeks after presentation she was walking with physical therapy assistance. 


\section{Poster 44}

\section{Acute Vision Loss and Malignant Hypertension in a Patient with Neurogenic Bladder}

Eric W. Moffet; Christina Lineback, Minjee Kim

Davee Department of Neurology, Northwestern University Feinberg School of Medicine, Chicago, IL, United States

\section{Background \& Purpose}

Here we present a case of reversible cerebral vasoconstriction syndrome (RCVS) caused by autonomic dysreflexia (AD) secondary to neurogenic bladder, which has been reported only once previously. The patient was an 18-year-old man with residual spastic quadriparesis from a traumatic $\mathrm{C} 6$ spinal cord injury. He presented with episodic thunderclap headache and vision loss associated with urinary incontinence. He suffered concurrent malignant hypertension, with systolic blood pressures into the $160 \mathrm{~s} \mathrm{mmHg}$ (baseline $90 \mathrm{~s} \mathrm{mmHg}$ ) and bradycardia, with heart rates into the 40s BPM (baseline $>60$ BPM). Neurologic examination revealed inability to count fingers in any visual quadrant and chronic quadriparesis.

\section{Methods}

Objective data was collected via serial neurologic examinations, head/neck CT angiography, and brain MRI. Intracranial vasospasm was assessed using transcranial doppler (TCD).

\section{Results}

He required monitoring in the neuro-ICU with a continuous nicardipine infusion due to episodic malignant hypertension and vision loss. CT angiography showed segmental arterial narrowing diffusely. MRI brain revealed bilateral parietal/occipital cortical and subcortical restricted diffusion and T2/FLAIR hyperintensities. TCDs displayed increased flow velocities of variable arteries over multiple days, with eventual resolution. He was diagnosed with RCVS and AD secondary to neurogenic bladder. Scheduled nimodipine was utilized to manage vasospasm. A foley catheter was placed and episodes ceased. He was discharged with no visual deficits.

\section{Conclusions}

AD presents as episodic hypertension and baroreflex-mediated bradycardia initiated by unrestrained sympathetic reflexes in a decentralized cord. It is often triggered by noxious, unperceived stimuli. AD persists until the stimulus is removed, while RCVS can be provoked by stressors. This case represents the second report of RCVS caused by neurogenic bladder-induced AD. Rapid recognition plus monitoring and management in the neuro-ICU led to complete recovery of acute neurologic deficits. Thus, in patients with subacute spinal cord injury presenting with new neurologic changes, AD must be considered. 


\section{Poster 45}

\section{Adjunctive N-Acetylcysteine for Carbon Monoxide Poisoning with Diffuse Cerebral Edema: A Case Report}

Casey A. Potts, Jan Bittar, Yousef Hannawi, Casey C. May, Keaton S. Smetana

Ohio State Wexner Medical Center, Department of Neurology, Columbus, OH, United States

\section{Background \& Purpose}

Carbon monoxide (CO) poisoning is currently a rare disorder that can result in severe injury to the central nervous system due to several mechanisms including production of reactive oxygen species (ROS). $\mathrm{N}$-acetylcysteine (NAC) is a potential adjunctive treatment due to its antioxidant properties. We report the successful treatment course of a patient with $\mathrm{CO}$ poisoning and severe cerebral edema using NAC.

\section{Methods}

This is a retrospective chart review of a single case.

\section{Results}

A previously healthy 22-year-old male suffered an accidental CO poisoning while warming up his snowpacked car. When Emergency Medical Services arrived, He was unresponsive with a Glasgow Coma Scale of 7. On arrival to the outside hospital, his carboxyhemoglobin ( $\mathrm{HbCO}$ ) level was $55.8 \%$. He was initiated on mechanical ventilatory support with administration of 100\% FiO2 and transferred to our facility for hyperbaric oxygen (HBO) therapy. Computed Tomography (CT) scan of the head revealed diffuse cerebral edema with maintained gray-white differentiation. He was managed with hyperosmolar fluid administration and HBO. Adjunctive NAC (Denver Protocol (150mg/kg load, followed by $210 \mathrm{mg} / \mathrm{kg} / \mathrm{day}$ )) was initiated to theoretically aid in reducing ROS. He received a total of two HBO therapies for a total duration of 210 minutes (120 minutes on first session and 90 minutes on second session). NAC was continued for 10 day duration. Brain MRI on day 5 showed no acute intracranial abnormalities and resolution of cerebral edema. He was discharged to inpatient rehabilitation on hospital day 30 and to home 6 days later.

\section{Conclusions}

We report the successful treatment of a patient with accidental $\mathrm{CO}$ poisoning and severe cerebral edema with $\mathrm{HBO}$ and NAC. Future research will need to consider the timing and duration of NAC in association of $\mathrm{HBO}$ sessions for optimal cognitive and functional outcomes of this rare condition. 


\section{Poster 46}

\section{Alternating Alpha and Spindle "Coma" in a Patient with Pontine Hemorrhage and Later Awakening}

Thanujaa Subramaniam, Aparna Vaddiparti, Jennifer Kim, Kevin N. Sheth, Lawrence J. Hirsch

Yale University, Department of Neurology, Division of Neurocritical Care, New Haven, CT, United States

\section{Background \& Purpose}

Alpha coma and spindle coma are two distinct electroencephalogram (EEG) patterns with a non-reactive background, previously described in isolation and are associated with various pathologies. Both patterns typically have poor outcomes when associated with brainstem lesions. We present a unique case of a patient with large pontine hemorrhage and EEG showing alternating alpha and spindle coma with apparent lack of reactivity.

\section{Methods}

Chart review.

\section{Results}

42-year-old woman found comatose with reactive pupils and posturing to painful stimuli. CT Head showed a large pontine hemorrhage extending into the fourth ventricle, without significant hydrocephalus. Continuous EEG performed on day 3 and day 12 showed two EEG patterns, alpha coma characterized by continuous $8-8.5 \mathrm{~Hz}$ waveforms and spindle coma, characterized by abundant $\mathrm{K}$ complexes and spindles. The two patterns alternated every 6-8 hours. Reactivity during EEG was assessed with visual, auditory and noxious stimuli with no reactivity during either pattern. The patient remained unresponsive until week 3 , after which she had spontaneous eye opening and consistently followed commands with vertical eye movements. Repeat two-hour EEG at this time showed continuous alpha waveforms. At 1 month, she exhibited voluntary finger grasps and toe movements.

\section{Conclusions}

We present a case with alternating alpha and spindle coma in a patient with pontine hemorrhage, who eventually followed commands. While isolated alpha and spindle coma describe a non-reactive EEG background to external stimuli, the transition between EEG patterns seen here possibly suggested an "intrinsic reactivity" or state changes, heralding her clinical improvement. This case also highlights the complexity of assessing reactivity and consciousness with a brainstem lesion affecting input to cortex; it is possible that applied stimuli did not transmit beyond the pons, thus, cortical reactivity wasn't adequately tested. 


\title{
Poster 47
}

\section{Atypical Posterior Reversible Encephalopathy Syndrome with Spinal Cord Involvement (PRES-SCI)}

\author{
Anna Finley Caulfield, Nick Murray, Elizabeth Tong, Jingjing Chen, Carl Gold, Bianca R. Palushaj, Nancy \\ Fischbein
}

Stanford University Department of Neurology and Neurological Sciences, Palo Alto, CA, United States

\section{Background \& Purpose}

Posterior reversible encephalopathy syndrome (PRES) is well-described and typically involves the parietal-occipital lobes, but may have atypical imaging features including spinal cord involvement.

\section{Methods}

Direct clinical observation and review of data.

\section{Results}

A 21-year-old woman with a history of celiac disease presented with one month of blurry vision and intermittent headaches. She was hypertensive $(230 / 130 \mathrm{mmHg}$ ) and tachycardic (140bpm).

Fundoscopic examination showed bilateral blurred disc margins, cotton wool spots, macular edema, mild arteriole attenuation, hemorrhages, and choroidal infarcts. Fluorescein angiography revealed retinal vascular (partial arterial) occlusion. Findings were concerning for hypertensive retinopathy or autoimmune vasculitis. Neurological exam noted poor visual acuity and red color desaturation (right > left) and symmetric hyperreflexia. Initial blood tests revealed an elevated BUN (35 mg/dL) and creatinine $(3.49 \mathrm{mg} / \mathrm{dL})$. MRI brain and spine revealed T2/FLAIR hyperintense signal in the bilateral orbital nerves and optic chiasm, as well as in the pons, medulla, and cervical (C1-C6) and thoracic (T1-T3) spinal cord, without restricted diffusion. Brain MRA and CSF tests were normal. All serum autoimmune tests returned normal, including neuromyelitis optica (NMO)/Aquaporin-4-IgG and MOG-FACS. Kidney biopsy demonstrated C3 sclerosing chronic glomerulonephritis. After hypertension control for two weeks, a repeat MRI brain and spine demonstrated near-resolution of T2/FLAIR hyperintensities.

\section{Conclusions}

PRES rarely manifests in the spinal cord. In case series, imaging of atypical PRES includes isolated deep gray nuclear, brainstem, cerebellar and, rarely, spinal cord involvement without cerebral hemispheric involvement. In the few reported cases of PRES-SCl, commonly in men presenting with severe hypertension and kidney failure, spinal cord expansile $\mathrm{T} 2$ hyperintensities were located at the cervical medullary junction and spanned four levels. Although this woman had optic, cervical, and thoracic involvement concerning for a demyelinating disorder, her tests were negative, and imaging improved with hypertension control alone. One month after presentation, her vision had improved and her headaches had resolved. 


\section{Poster 48}

\section{Autoimmune Encephalitis in a 31-year-old Male with Myasthenia Gravis and COVID-19}

Max Kazer, Victoria L. Wu, Jenelle P. Raynowska, Dominic Ferrey, Anastasie Dunn-Pirio, Jamie Nicole LaBuzetta

University of California, San Diego Department of Neurosciences, San Diego, CA, United States

\section{Background \& Purpose}

Cases of autoimmune encephalitis (AE) have been rarely reported in patients with myasthenia gravis (MG). We present a case of concurrent AMPA and CRMP-5 antibody autoimmune encephalitis in a patient with seropositive MG found to have COVID-19.

\section{Methods}

We performed retrospective review of the patient's electronic health record and evaluated the current literature.

\section{Results}

A 31-year-old male with newly diagnosed acetylcholine receptor positive MG and thymoma requiring prednisone, plasma exchange (PLEX), and eculizumab infusion for outpatient management presented with two weeks of progressive disorientation and psychosis. Following admission, he was intubated for myasthenic crisis and received a complete course of intravenous immunoglobulin. COVID-19 PCR returned positive, warranting treatment with remdesivir and dexamethasone. In evaluating his cognitive symptoms, MRI brain was unremarkable and electroencephalogram was without epileptiform abnormalities. Cerebrospinal fluid (CSF) results showed 21 white blood cells ( $90 \%$ lymphocytes), but were otherwise unremarkable. Further history revealed that the patient was overmedicating with prednisone, so his steroids were decreased. Unfortunately, his neurologic exam deteriorated and he developed autonomic storming. Repeat MRI brain revealed hippocampal atrophy. He was treated with nine sessions of PLEX for presumed autoimmune encephalitis and CSF tests ultimately revealed AMPA-R (1:256) and CRMP-5 IgG (1:128) antibodies, which were felt to be related to his thymoma. Given persistently poor neurological function, he underwent resection of his thymoma followed by treatment with rituximab. His condition stabilized and he was discharged to a long-term care facility with plans for continued rituximab infusions and outpatient follow-up.

\section{Conclusions}

AMPA-R and CRMP-5 autoantibodies are associated with tumors such as thymoma. We theorize that our patient's autoantibodies and autoimmune encephalitis were triggered by COVID-19 infection. In patients with MG and thymoma who develop new psychosis, autoimmune/paraneoplastic encephalitides should be considered on the differential. 


\title{
Poster 49
}

\section{Candida Auris Meningitis due to External Ventricular Drain in a Patient with Severe COVID-19 Pneumonia}

\author{
Omar Rahman, Asma Siddiqui, Lori Wetmore, Storm Van Wey, Piiamaria Virtanen, Daniel Perkins, Sierra \\ Tackett, Farhaan Vahidy
}

Indiana University Health Methodist Hospital, Critical Care, Indianapolis, IN, United States

\begin{abstract}
Background \& Purpose
Candida auris is a multidrug-resistant fungal pathogen associated with invasive infections and candidemia, with associated mortality of $30-60 \%$. Colonization is on the skin particularly in axilla and other skin folds. CNS infection is very rare. We present the first case of candida auris meningitis due to an external ventricular drain placed in a patient with active COVID-19 infection.COVID-19 related fungal co-infection is a rising worldwide concern and reports of candida auris outbreaks in SARS CoV-2 patients show mortality is greater than $50 \%$ for such patients.
\end{abstract}

\section{Methods}

Chart Review

\section{Results}

28 y-o man with a history of ventricular-peritoneal shunt (VPS) for hydrocephalus since childhood and schizophrenia was admitted for altered mental state and seizure-like activity. Extensive workup was done to rule out infections, autoimmune and paraneoplastic causes. Severe catatonia was considered for which he underwent electroconvulsive therapy. Additionally his CT head showed hydrocephalus. An attempt to replace VPS was unsuccessful. An external ventricular drain (EVD) was placed.During the prolonged inpatient stay, he developed severe hypoxemic respiratory failure and diagnosed with COVID19 pneumonia. He received remdesivir and dexamethasone. Steroids were given for 10 days following which he developed worsening mental status and fever. Cultures were sent including the cerebrospinal fluid (CSF) that grew candida auris. He was treated with intrathecal caspofungin for 5 days, intravenous amphotericin plus oral flucytosine for 7 days and voriconazole for 6 weeks. Repeat CSF cultures were negative and VPS was replaced. He was subsequently discharged to rehabilitation.

\section{Conclusions}

Repeat CSF cultures were negative and VPS was replaced. He was subsequently discharged to rehabilitation.Post-covid fungal infections are rising due to immune suppressive therapy as well as prolonged hospitalizations. Candida auris is increasingly recognized as a lethal post-covid organism. We describe the first case of EVD-related candida auris meningitis in a covid-19 patient with obstructive hydrocephalus. Successful clearance of the organism was achieved with broad spectrum anti-fungal therapy including intrathecal echinocandin administration. 


\section{Poster 50}

\section{Cardiac Cephalalgia: Report of 4 Cases}

Hitoshi Kobata, Akihiro Kambara, Takayoshi Negoro, Makiko Kawakami

Osaka Mishima Emergency Critical Care Center, Takatsuki, Japan

\section{Background \& Purpose}

The International Classification of Headache Disorders-III classifies cardiac cephalalgia as one of the headaches caused by impaired homeostasis. Migraine-like headache occurring on exertion is the typical manifestation, whereas thunderclap headache might rarely develop. We experienced four cases of cardiac cephalalgia that fulfilled diagnostic criteria, two of which mimicked subarachnoid hemorrhage (SAH).

\section{Methods}

Single-center retrospective chart review during 12 years.

\section{Results}

Case \#1: A 69-year-old man with a history of percutaneous coronary intervention ( $\mathrm{PCl}$ ) developed a sudden severe occipital pain, followed by nausea and cold sweat. Coronary angiography (CAG) revealed occlusion of the right coronary artery (RCA), and he underwent PCI.Case \#2: A 66-year-old woman complained of increasing occipitalgia and chest discomfort while riding a bicycle. CAG demonstrated 99\% stenosis of the left anterior descending artery (LAD), and PCI was performed.Case \#3: A 54-year-old man felt faintness and cold sweating, followed by strangulating occipitalgia after lunch. CAG detected occlusion of the RCA, and PCI was performed.Case \#4: A 72-year-old man went into shock after complaining of a sudden severe headache and nausea. Emergency CAG was conducted while administering vasopressors. Three vessel disease was detected, and he had PCI for RCA, which showed 99\% stenosis. He underwent intra-aortic balloon pumping and transferred for cardiac surgery. In all four patients, electrocardiograms showed ST-segment elevation or depression, and echocardiograms revealed wall motion abnormality. Emergency head CT scans showed no SAH and the headache was relieved after $\mathrm{PCl}$.

\section{Conclusions}

Case \#1 and \#4 presented with thunderclap headaches mimicking SAH. Because SAH patients may present neurogenic myocardial stunning mimicking acute coronary syndrome, recognition of cardiac cephalalgia is crucial. Evaluation of cardiac function is mandatory in SAH-suspected patients in an emergency setting. If the patients had cardiac cephalalgia, timely treatment for culprit lesions in the coronary arteries should be carried out for life-saving. 


\section{Poster 51}

\section{Cardiac Findings In Neurocritical Care Patients with COVID-19}

Denise Michele Rios, Stephanie Molidor McGovern, Shaheryer Hafeez

UT Health San Antonio; Department of Neurosurgery, San Antonio, Texas, United States

\section{Background \& Purpose}

Acute cardiac dysfunction with new onset cardiomyopathy in patients with severe SARS-CoV- 19 (COVID-19) has been described. Manifested as heart failure with/without hemodynamic instability. However, COVID-19 cardiomyopathy has not been described in patients with Neurocritical care disorders.

\section{Methods}

We retrospectively analyzed 26 consecutive adult patients admitted to the Neurosciences Critical Care Unit (NSICU) who were also positive for COVID-19. We collected detailed data on echocardiogram reports and EKGs.

\section{Results}

$30 \%$ of our patients were found to have cardiovascular manifestations or cardiomyopathy (8 of 26). Severe hypokinesis (EF <25\%, PASP $33 \mathrm{mmHg}$ ) was seen in 4 patients presenting with a stroke. Mild hypokinesis (LVEF average 33\%, PASP $31 \mathrm{mmHg}$ ) was seen in 4 other patients; Seizure $(n=1)$, Subarachnoid hemorrhage $(n=1)$, Traumatic brain injury $(n=1)$, and stroke $(n=1)$. Their past medical history included hypertension $(n=6)$, coronary artery disease $(n=2)$ and cardiovascular disease $(n=2)$. The majority of these patients had slight increase in troponins with no abnormal EKG changes on admission but developed dilated cardiomyopathy within 48 hours of their admission. Mortality was seen in $37 \%$ of the patients.

\section{Conclusions}

Dilated cardiomyopathy (DCM) can be seen in up to $30 \%$ of patients admitted to the NSICU patients with COVID-19. We recommend screening all patients with COVID-19 with an echocardiogram and daily EKGs. 


\section{Poster 52}

\section{Case Report of Anti-thymocyte Globulin Use After Chimeric Antigen Receptor T-cell Therapy}

Melanie Goodberlet, Mary Amatangelo, Matthew Bevers, Melanie Goodberlet, Kaylee Marino, Michael Schontz, Helen Wang

Brigham and Women's Hospital - Department of Pharmacy, Department of Neurology, Boston, MA, United States

\section{Background \& Purpose}

This case report describes our experience utilizing equine anti-thymocyte globulin (ATG) for treatmentrefractory immune-effector cell-associated neurotoxicity syndrome (ICANS) and cytokine release syndrome (CRS) secondary to chimeric antigen receptor (CAR) T-cell therapy. ATG is theorized to dampen CAR-T cell levels and reduce cytokine storm. However, ATG effectively reverses the therapeutic effects of CAR-T therapy. Currently, there is limited data detailing the use of ATG for this indication.

\section{Methods}

Data was collected from the electronic health record.

\section{Results}

A 36-year-old female with refractory mantle cell lymphoma was admitted for CAR-T therapy complicated by treatment-refractory ICANS and CRS. On day 5 after CAR-T infusion, she developed neurotoxicity and CRS, which manifested as fevers and altered mental status. She was initially managed with high dose-steroids and tocilizumab. Neurologic exam and respiratory function declined requiring intubation and ICU care on day 8 where the patient was started on anakinra. The patient had no improvement in aroUnited Statesl, did not follow commands, and was not tracking which prompted medical treatment for diffuse cerebral edema. Equine ATG was administered at 2 milligrams per kilogram once on day 8 . However, imaging and exam showed loss of intracranial flow and concern for brain death. In the evening of hospital day 8 , the patient was transitioned to comfort measures and passed.

\section{Conclusions}

Multimodal immunomodulatory therapy is important for managing ICANS and CRS secondary to CAR-T therapy. ATG may be considered for refractory cases but it may be most efficacious when administered earlier in the treatment course. Additionally, providers should weigh the risks and benefits of use. 


\title{
Poster 53
}

\section{Case Series: Successful Treatment of Refractory NMDAr Encephalitis with Intrathecal Rituximab}

\author{
Nicolas Chiriboga, Torrey Baines, Charlene Pringle, Suman Ghosh, Renee Modica, Melissa Elder
}

University of Florida College of Medicine, Department of Pediatrics, Gainesville, FL, United States

\section{Background \& Purpose}

NMDAr encephalitis can be a challenging disease to treat, with described instances in which patients have been refractory to standard immunotherapy. We describe two female pediatric patients refractory to aggressive immunosuppression and teratoma resection but improved after intrathecal rituximab.

\section{Methods}

Clinical information was extracted from chart review utilizing EHR.

\section{Results}

A 13 yo and a 17 yo female presented to our institution for treatment of refractory NMDAr encephalitis. Both initial presentations were for seizures, dysautonomia, and behavioral changes; and had been treated with PLEX, IVIG and steroids without improvement. Due to chronic respiratory failure, both had received a tracheostomy and were mechanically ventilated. Immunomodulating therapies including rituximab, bortezomib, and cyclosporine were administered given the severity of their disease. Maintenance plasmapheresis was continued on a weekly schedule, as were steroids. MRI of the pelvis confirmed ovarian teratomas and oophorectomies were performed in both patients, resulting in transient improvement. However, the two patients had plateauing and then worsening of symptoms; as well as increasing NMDAr antibody titers, so aggressive immunotherapy was restarted. The 13 yo patient started having seizures and developed suprarefractory status epilepticus, which required pentobarbital coma to achieve burst suppression. IV anakinra was added to her immunotherapy regimen. In both cases, it was decided to provide intrathecal rituximab therapy, $25 \mathrm{mg}$ weekly for 4 weeks. After this, both were noted to have significant clinical and neuro-cognitive improvement; and significant reductions in serum and CSF NMDA receptor antibodies. Neither of the patients had any of the described complications of IT rituximab. Both were able to be discharged to rehabilitation centers. On follow up visits, both continued to show significant neuro-cognitive improvement.

\section{Conclusions}

It is known that only about $0.1 \%$ of rituximab penetrates the blood brain barrier. There are few case reports and case series reporting the successful use of intrathecal rituximab for refractory NMDA receptor encephalitis. Ours is the first pediatric case series of this type. This case series highlights the safety and efficacy of intrathecal rituximab for refractory severe NMDAr encephalitis in conjunction with systemic immunotherapy 


\title{
Poster 54
}

\section{CJD Mimicking PERM: Glycine-R Autoantibodies in CJD}

\author{
W. David Freeman, Ashley Rogers, Evelyn Lazar, Lisette Dominguez, Perry Bechtle, Lauren Ng, Gregory \\ Day,
}

Mayo Clinic Neurosciences Intensive Care Unit, Jacksonville, FL, United States

\section{Background \& Purpose}

Creutzfeldt-Jakob disease (CJD) and autoimmune encephalitis with antibodies against neuronal surface antigens may have a similar presentation of rapidly progressive dementia. While management of CJD is supportive and palliative, autoimmune encephalitis treatment can significantly improve or halt the progression of the disease. Correctly differentiating between these conditions is important as it is crucial in determining management.

\section{Methods}

Review of the literature using National Library of Medicine/Google Scholar search engines using search terms of Creutzfeldt-Jakob disease, Glycine Receptor antibody, neuronal surface antigen autoimmune encephalitis.

\section{Results}

We present a case of rapidly progressive dementia in a 49 year old female with 6-week decline including a progression from frequent falls with ataxia to progressive personality change with hemibody dystonia and myoclonus, rigidity and eventual obtunded state requiring intubation. Given young age and rapid onset, autoimmune encephalitis (including progressive encephalomyelitis with rigidity and myoclonus (PERM) with neuron surface antigen Glycine Receptor alpha 1 antibody) was considered with empiric steroids were initiated while testing for prion disease was pending. Comprehensive workup did not reveal infectious cause but was remarkable for positive serum Glycine Receptor alpha 1 antibody giving her the presumptive diagnosis of PERM. Plasma exchange therapy was completed without improvement in her condition. Subsequently, CSF Glycine Receptor alpha 1 antibody results were negative and testing from National Prion Disease Pathology Surveillance Center confirmed diagnosis of CJD with positive RTQuIC, positive 14-3-3 and t-tau above the level of quantification (>20,000 pg/mL). Patient was transitioned to hospice.

\section{Conclusions}

Rapidly progressive dementia presents diagnostic challenges given the time delay in testing results which has implications on prompt detection and management of autoimmune encephalopathies. The presence of clinically irrelevant neuronal antibodies in prion disease makes interpretation complex and challenging giving incorrect presumptive diagnosis of autoimmune encephalitis. 


\section{Poster 55}

\section{CNS Vasculitis with Diffuse Infarcts, SAH and IVH Related to COVID-19}

Jingxin Wang, Sean Marinelli, Jessica Magid-Bernstein, Sachin Agarwa,; Roh David, Jan Claassen

Columbia University Irving Medical Center, New York, NY, United States

\section{Background \& Purpose}

Neurologic involvement of COVID-19 are uncommon, among which mostly reported include encephalopathy, large vessel stroke and polyneuropathy. The underlying mechanism of cerebral damage remains unclear. Some hypothesis propose direct viral invasion of the vessel wall causing endotheliopathy. Here we report a unique neurologic manifestation of COVID-19 in a patient who presented with extensive cerebral vasculitis in large vessels with infarcts and diffuse subarachnoid hemorrhage (SAH) with intraventricular hemorrhage (IVH).

\section{Methods}

Data was collected by inpatient encounter.

\section{Results}

A 60-year old patient with past medical history of hypertension and type 2 diabetes presented to our institution with cough, fever and myalgia on December 10, 2020. He was soon intubated for hypoxic respiratory failure due to acute respiratory distress syndrome. After prolonged 6 weeks of sedation and paralysis, he received tracheotomy. 4 days off sedation, he was still comatose and developed anisocoria. Emergent head CT scan was performed and showed multifocal acute to subacute infarcts of the right MCA and PCA watershed territories; multifocal SAH in interpeduncular cistern, right suprasellar cistern and left more than right sylvian fissures; hydrocephalus with IVH in bilateral ventricles. CT angiogram demonstrated irregular vessels especially in basilar artery and bilateral vertebral arteries, however no aneurysm or dissection was found. DSA performed same day revealed significant morphological changes of multiple vascular territories including right supraclinoid ICA, right M1 and A1 segments, and bilateral SCAs. TCD showed increased Lindegaard ratio in both MCAs with increased mean velocities, R>L. LP was performed without abnormal findings that indicated underlying infectious meningitis or encephalitis. Autoimmune and paraneoplastic panels were also negative. Endocarditis was ruled out by TEE. Patient was treated with pulse steroids with slow taper. Vessel wall imaging was performed weeks later showed concentric enhancement. His mental status continued to improve and he was able to follow simple commands when discharged to LTAC.

\section{Conclusions}

Vasculitis and vasculopathy could be a rare CNS manifestation of COVID-19. 


\title{
Poster 56
}

\section{Cocaine Use and Toxic Leukoencephalopathy}

\author{
Nora Ko, Jennifer Axelband, Jonathan Hosey
}

St. Luke's University Health Network Neurology Residency, Bethlehem, PA, United States

\section{Background \& Purpose}

Toxic leukoencephalopathy (TL), an uncommon condition of white matter injury secondary to neurotoxin exposure, is not typically seen with cocaine use. TL secondary to drugs of abuse often occurs with opiates. The diagnosis is made on history of toxin exposure, impaired cerebellar function, and imaging findings such as symmetric white matter lesions of the cerebellum and posterior cerebrum.

\section{Methods}

Chart review for case report presentation

\section{Results}

28-year-old male presented with severe headache, nausea, emesis and unsteady gait 3 days after inhaling cocaine. He had an insignificant medical history and was not taking any medications. He was afebrile with normal vital signs. Physical examination was unremarkable except for left upper extremity edema. Laboratory abnormalities included rhabdomyolysis associated with transaminitis. CSF studies were unremarkable. Bacterial and viral culture of CSF, including HIV, were negative. Lymphoma/leukemia and autoimmune panels were negative. Toxicology screen was negative for opiates and cocaine. Computerized tomography (CT) revealed diffuse bilateral symmetric cerebellar hypodensities with evidence of fourth ventricle compression. CTA excluded vertebral dissections. CT chest abdomen and pelvis demonstrated evidence of fluid collections within the musculature consistent with prolonged lateral recumbency. Magnetic resonance imaging demonstrated T2 signal intensity of the bilateral cerebellar hemispheres with diffusion restriction but no correlate on apparent diffusion coefficient. On fluid-attenuated inversion recovery images there was a hyperintense signal of the bilateral cerebellar hemispheres. Obstruction of the cerebral aqueduct and dilation of the lateral and third ventricles with $5 \mathrm{~mm}$ of tonsillar descent was apparent. Cerebral angiogram showed no evidence of vasculopathy. An external ventricular drain was placed which improved headache, nausea and emesis. He was treated with intravenous methylprednisolone for 5 days. Re-Imaging prior to discharge revealed improvement in edema and hydrocephalus. Discharge occurred 9 days after admission. The patient reported no recurrence of symptoms at his 8 week follow-up.

\section{Conclusions}

In TL, a latent period related to toxin absorption or persistent metabolic change may occur between exposure and symptom development known as "coasting". A comprehensive social history and early recognition of TL secondary to substance abuse is critical. Prompt toxin removal, supportive therapy, and corticosteroid administration may aid in recovery. 


\title{
Poster 57
}

\section{Covid-19 Associated New-onset Refractory Status Epilepticus (NORSE): A Case Report}

\author{
Haoming Pang, Michael Gezalian, Shahed Toossi, Maranatha Ayodele, Shouri Lahiri
}

Cedars-Sinai Medical Center, Departments of Neurology and Neurosurgery, Los Angeles, CA, United States

\section{Background \& Purpose}

Although there have been few reported cases of new onset seizures reported in patients with COVID-19, even rarer is a new-onset refractory status epilepticus (NORSE) associated with COVID-19. We present such a case in order to further understand the neurological manifestations of COVID-19.

\section{Methods}

Data collected via retrospective chart review.

\section{Results}

A 55-year-old male presented with COVID-19 pneumonia. Hospital course notable for by sepsis and deep vein thrombosis (DVT) requiring anticoagulation. Early in his hospital course, he required sedation for acute respiratory distress syndrome related to COVID-19 pneumonia, however, he remained encephalopathic after discontinuation of sedation. Initial head computed tomography was unremarkable. Patient gradually regained consciousness and was interactive. Two-weeks later, he developed generalized seizures. Subsequent neuroimaging revealed subarachnoid hemorrhage in the left sylvian fissure and within the sulci in the left frontal lobe. Two days after this event, patient developed additional seizures that were refractory to benzodiazepines and levetiracetam. Anti-epileptic drugs were escalated to lacosamide, phenytoin, midazolam infusion, propofol, and eventually ketamine and pentobarbital infusion. Electroencephalogram revealed left frontotemporal periodic lateralized epileptiform discharges and occasional rhythmic runs of delta and theta activity with evolution consistent with subclinical seizures. Cerebrospinal fluid studies and MRI brain were unrevealing. Patient was treated with 5 days of solumedrol and IVIG. Eventually, seizure control was achieved and on day 28 since super-refractory status epilepticus onset, patient was following axial commands.

\section{Conclusions}

COVID-19 is thought to enter the central nervous system through the olfactory bulb, which may result in neurological manifestations including NORSE. Although our patient developed subarachnoid hemorrhage, possibly from anticoagulation, the refractory nature of his seizures appear to be out of proportion to the extent of subarachnoid hemorrhage demonstrated on neuroimaging, leading us to conclude that NORSE occurred due to COVID-19 infection. 


\title{
Poster 58
}

\section{Cryptococcus Neoformans Meningoencephalitis in an Immunocompetent Adult}

\author{
Elo Ibekwe, Tamara Strohm
}

The Ohio State University, Department of Neurology, Columbus, $\mathrm{OH}$, United States

\section{Background \& Purpose}

To describe the diagnosis and management of Cryptococcal meningoencephalitis in an immunocompetent patient. Cryptococcus neoformans most commonly causes meningoencephalitis with elevated ICP in profoundly immunocompromised individuals. Mortality is high at 20-30\%. Uncontrolled diabetes mellitus and alcoholism may be associated with infection.

\section{Methods}

\section{$\mathrm{N} / \mathrm{A}$}

\section{Results}

This is a 73-year-old Caucasian male with a history of hypertension, and diabetes mellitus (A1c 6.7) who presented as a transfer from an outside hospital after one month of cognitive decline and headache. CT scan there revealed hydrocephalus with transependymal edema. Upon arrival, he was afebrile with GCS 9. MRI brain with and without contrast showed communicating hydrocephalus with periventricular and leptomeningeal enhancement within the basal cisterns, brainstem, and sylvian fissures. CSF analysis revealed xantochromia with opening pressure of $14 \mathrm{~cm} \mathrm{H20}$, protein of $770 \mathrm{mg} / \mathrm{dL}$, glucose of $63 \mathrm{mg} / \mathrm{dL}$, and WBC of 118 (20\% / 77\% of neutrophils / lymphocytes respectively). CSF culture grew Cryptococcus neoformans. Infectious and malignancy workup was unremarkable. There was no evidence of alcoholic cirrhosis. He was treated with amphotericin B and fluocytosine, transitioned to fluconazole after 15 days given profound neutropenia. Furthermore, he underwent emergent EVD placement with opening pressure $4 \mathrm{cmH} 20$. Unfortunately, he developed acute right hemiparesis and MRI revealed acute ischemic strokes in the pons and cerebellum. He was maintained on aspirin and statin therapy. His EVD was removed after 21 days. He improved slowly with some residual cognitive difficulty. On hospital day22, we recommended PEG tube placement which the family declined. The patient was transitioned to hospice care and expired several days later.

\section{Conclusions}

Here we report delayed diagnosis of Cryptococcal meningoencephalitis in the immunocompetent adult with normal opening pressure. Prompt diagnosis, administration of antifungal agents, and CSF diversion is essential to prevent irreversible damage and death. 


\section{Poster 59}

\section{Diagnostic Dilemma in the Age of Immunosuppression}

Manmeet Kaur, Angela Hays-Shapshak, Ravipaul Singh Virdi, Rhodemarie Maron

University of Alabama at Birmingham, Birmingham, AL, United States

\section{Background \& Purpose}

NPSLE can be the presenting feature or seen during Lupus exacerbations. NPSLE are hypothesized to be mainly autoimmune mediated, but rare entity of disease manifestation from immune suppressive medications and opportunistic infections should be considered, especially when presentation is atypical

\section{Methods}

We present a 30 YOF diagnosed with SLE at the age of 22 with recurrent serositis, digital ischemia and deep vein thrombosis maintained on Prednisone and Mycophenolate mofetil. She developed headache after renal biopsy, and brain imaging obtained was conce

\section{Results}

Brain biopsy: Pattern of demyelination consistent with ADEM. Stains for Epstein - Barr virus (EBV) were negative, ruling out lymphoproliferative disease and EBV-induced ADEM.

\section{Conclusions}

ADEM is a rare severe complication in SLE. Myriad of immune system abnormalities in SLE trigger abnormal inflammatory reaction against the myelin sheath. The unique aspect in the case is occurrence of ADEM in immunosuppressed state with imaging findings atypical of ADEM, rather raising concern for lymphoma or lymphoproliferative disease which has treatment implications. While ADEM is reported as a rare NPSLE manifestation, patients on chronic immunosuppression should be evaluated for lymphoproliferative disease and opportunistic infections. 


\section{Poster 60}

\section{Diaphragmatic Pacing for Brain Stem Lesion-Related Respiratory Failure}

GM Horstman, MJ Elmo, MA De Georgia, RP Onders

University Hospitals Cleveland Medical Center/Case Western Reserve University, Cleveland, $\mathrm{OH}$, United States

\section{Background \& Purpose}

Diaphragmatic pacing (DP) has been used extensively in cases of respiratory failure from high cervical cord injury, leading to decreases in morbidity, mortality, and length of hospital stay. Although DP has been used in congenital hypoventilation syndrome, it has not been well studied in adults with secondary causes of centrally driven respiratory failure. We present three novel cases of DP in the treatment respiratory failure secondary to brain stem lesions.

\section{Methods}

Cases of respiratory failure secondary to brainstem lesions were identified in the diaphragmatic pacing database with additional information obtained through chart review.

\section{Results}

Case \#1: Neuromyelitis optica (NMO). 21-year-old woman with aquaporin-4 antibody positive NMO with demyelinating lesions in the area postrema and cervico-medullary junction. This resulted in severe central sleep apnea and chronic hypercapneic respiratory failure necessitating frequent BiPAP use. With DP placement and continued immunosuppressive therapy, the frequency of BiPAP use decreased and chronic hypercapnia improved. Case \#2: NMO. 27-year-old man with seronegative NMO with extensive demyelinating lesions in the lower pons, medulla, and C2-7 cervical cord resulting in respiratory failure requiring intubation. With DP placement 11 days after intubation and continued immunosuppressive therapy, he was weaned from the ventilator one month later. Case \#3: Medullary Infarct. 53-year-old man with a right medullary infarct secondary to a right vertebral artery occlusion resulting in respiratory failure. He underwent DP placement three months after intubation and was weaned from the ventilator three days later. He continues to require DP for persistent intermittent apnea.

\section{Conclusions}

This is the first report of DP use in the treatment of respiratory failure due to brain stem lesions. Patients in the neuro-ICU should be screened for central hypoventilation and central sleep apnea. Earlier initiation of DP should be considered in this patient population to reduce the incidence of superimposed ventilator-induced diaphragmatic dysfunction. 


\section{Poster 62}

\section{Disseminated Cryptococcus infection with Meningoencephalitis in an Immunocompetent Host}

Aimalohi Esechie, Aimalohi Esechie, Sandeep Bhatt, Joshua M. Peterson, Marisa C. Nielsen, Ping Ren, Kinjal Desai, Alok Dabi

University of Texas Medical Branch, Department of Neurology, Galveston, TX, United States

\section{Background \& Purpose}

Cryptococcal meningoencephalitis is a significant cause of morbidity and mortality in immunocompromised patients worldwide, with rare case reports in immunocompetent patients. Here we present a case of disseminated Cryptococcus gattii (C. gatti) with severe meningoencephalitis in an immunocompetent patient, the first such case in Texas to our knowledge.

\section{Methods}

Literature search was done.

\section{Results}

Case: A 40-year-old male with a history of migraine presented with new-onset status epilepticus. He was intubated on-site, with subsequent treatment of intravenous levetiracetam, lacosamide and propofol infusion. The patient had a history of 5-month progressive headache, cervicalgia, nausea, vomiting and unintentional 20 pounds weight loss, with an inconclusive workup.Post admission, following fever development, his cerebrospinal fluid (CSF) demonstrated neutrophilic pleocytosis, and fungal culture grew C. gattii. He was treated with intravenous $L$-Amphotericin-B with Flucytosine induction therapy. Repeat CSF exam demonstrated persistently elevated opening pressure, up to $55 \mathrm{~cm}$ of water and recurrent yeast presence.Brain MRI demonstrated diffuse symmetric cortical diffusion restriction with T2/FLAIR hyperintensity, sulcal effacement and foci of diffusion restriction in the bilateral cerebellar hemispheres. CT thorax showed a left-sided consolidation but with negative fungal culture on bronchoalveolar lavage. Bone marrow biopsy identified rare extracellular organisms morphologically similar to Cryptococcus. Extensive investigation for occult immunocompromised state was negative. The patient died on Hospital day 13 after initiation of palliative care following family discussion.

\section{Conclusions}

Cryptococcus infection is rare, and much more so in immunocompetent patients. Disseminated Cryptococcus gatti infection is much less common than C. neoformans. Our case represents the need for continued vigilance about this aggressive infection with a potentially fatal outcome, even in immunocompetent patients. 


\title{
Poster 63
}

\section{Electrographic Abnormalities Recorded in a Patient with NMDA-R Encephalitis Using Depth and Subgaleal Electrodes}

\author{
Asma Zakaria, James Leiphart, Allen Waziri, Ronald Emerson, Laith Altaweel \\ Inova Fairfax Hospital, Department of Medicine- Critical Care, Falls Church, VA, United States
}

\section{Background \& Purpose}

EEG in patients with NMDA-R encephalitis typically demonstrates severe slowing along with a characteristic pattern of delta brush-like activity. Clinical seizures are common but are often unaccompanied by scalp-recorded ictal EEG patterns, perhaps due to emanation from regions inaccessible to scalp recording. We describe a patient with NMDA-R encephalitis exhibiting clinical seizures, positive ictal SPECT and negative scalp EEG in whom electrographic seizures were detected by an intracranial depth electrode as well as subgaleal electrode arrays.

\section{Methods}

Subgaleal electrode arrays ( 4 bipolar parasagittal channels per side) were placed at the bedside using a trocar-and-sheath device. Eight-contact depth electrodes were placed at the bedside within right parietal and temporal regions using a disposable twis

\section{Results}

The patient experienced repeated clinical seizures, manifest by intermittent agitation and clonic movements. There were no correlative electrographic abnormalities visualized with standard scalp EEG. We did however observe clear $3 \mathrm{~Hz}$ rhythmical discharges recorded from the inferomedial contacts on the temporal depth electrode that, along with clinical seizures, responded to progressive treatment. Also noted were high (gamma) frequency bursts lasting 1-10 seconds, occurring within a background of lower amplitude delta activity. Prominent delta brush-like activity was evident in scalp, subgaleal and depth electrodes but was most striking in the subgaleal electrodes. Subgaleal electrodes also demonstrated markedly less artifact than concurrent scalp EEG.

\section{Conclusions}

In patients with NMDA-R encephalitis, intracranial depth or subgaleal electrode recording may be useful for detection of electrographic seizure activity that is typically not evident in routine scalp recordings. In addition to best demonstrating the delta brush-like pattern characteristic of NMDA-R encephalitis, subgaleal electrodes appear to provide resistance to artifacts that commonly contaminate prolonged recordings in the ICU. 


\title{
Poster 64
}

\section{Encephalitis in a 33-year-old Woman Following J\&J COVID-19 Vaccination}

\author{
Eunice J. Lee, Lucy G. Maguire, Omar Syed Shah
}

Thomas Jefferson University Neurocritical Care Department, Philadelphia, PA, United States

\section{Background \& Purpose}

Historically, meningoencephalitis and demyelinating disease have been reported following vaccinations(5-6). To date, there have been multiple reported cases of post-infectious encephalitis in COVID-19 patients(1-4), however no cases of COVID 19 vaccination associated encephalitis.

\section{Methods}

Patient information was obtained via chart review. A literature search was conducted using any combination of terms "vaccine", "COVID", "encephalitis" and "neurological disease."

\section{Results}

33-year-old woman with a history of hypothyroidism, PANDAS on IVIg, Celiac and Lyme disease who presented to the hospital with progressive encephalopathy. On April 9th, 2021, the patient received the J\&J COVID-19 vaccine. Ten days later, she developed a severe headache, lethargy, and became nonverbal. On exam, she was obtunded with bilateral lower extremity hyperreflexia and positive Babinski signs. She required intubation due to altered mental status. Initial LP showed lymphocytic pleocytosis with elevated protein. cEEG demonstrated left hemispheric focal slowing and occasional GRDA, along with diffuse background slowing. MRI Brain revealed extensive symmetric bilateral hyperintensity with enhancement, primarily involving the deep and subcortical white matter with sparing of the cortices. There was also focal enhancement in the left medial temporal lobe. Differential included postinfectious/autoimmune encephalitis, toxic-metabolic injury or MOG inflammatory/autoimmune encephalitis. During her hospitalization, she received antimicrobials, 5 days high dose pulse steroids, plasmapheresis (5 sessions) and IVIg without improvement. Repeat LP was negative for other infectious and autoimmune studies (e.g., Paraneoplastic, MOG-Ab, NMO/AQP4). Repeat MRI brain showed similar hyperintensity with interval improvement in enhancement.

\section{Conclusions}

There is no direct evidence the J\&J COVID-19 vaccine may have caused this patient's encephalitis. However, as J\&J uses a viral vector compared to the other mRNA vaccines, it may have played a role in inciting this in a patient with a history of autoimmune conditions. 


\title{
Poster 65
}

\section{Enhancing Chronic Subdural Hygroma Mimicking Acute Subdural Hematoma after Intravenous Contrast Injection}

Kenneth Sherry, Rochelle Sweis

\author{
Advocate Christ Medical Center Neurocritical Care, Oak Lawn, IL, United States
}

\section{Background \& Purpose}

Contrast extravasation mimicking intracerebral hemorrhage on non-contrast computed tomography (CT) of the brain is a common finding after intra-arterial injection of iodinated contrast for cerebral angiography. There is limited evidence of contrast enhancement of subdural effusions after both cerebral and coronary angiography and no published cases of enhancement of subdural effusions after intravenous injection of contrast dye mimicking subdural hemorrhage.

\section{Methods}

Case Report

\section{Results}

The patient is an 88-year-old male with a history of pulmonary embolism on apixaban, hypertension, and frequent falls. He became suddenly unresponsive at home and presented with concern for large vessel occlusion. Non-contrast CT brain demonstrated bilateral subdural hygromas. CT angiography of the head and neck and CT perfusion was unremarkable. He was intubated for airway protection and sedated. His anticoagulation was not reversed. A follow up non-contrast CT brain was performed two hours later, demonstrating acute to subacute bilateral subdural hematomas. Since there was no significant increase in volume or recent trauma and his neurologic exam was improved off sedation, reversal of his anticoagulation was deferred. A follow up non-contrast CT brain twenty-four hours later demonstrated resolution of the hyperattenuated fluid collection with stable size.

\section{Conclusions}

Uptake of contrast into subdural effusions is a rare but known complication after injection of gadolinium-based contrast and intra-arterial contrast studies. Mimicking an acute subdural collection can result in unwarranted intervention. A careful review of $\mathrm{CT}$ attenuation values, monitoring of the evolution of the effusion, taking the entire clinical picture into account with correspondence of the neuroradiology experts can be useful discriminators and assist with appropriate decision making. 


\section{Poster 66}

\section{False Positive Ancillary Brain Death Testing in Post Cardiac Arrest}

Monica Noya Santana, Shane Dorsey, Aimee Aysenne

Tulane University, School of Medicine, Department of Clinical Neurosciences, New Orleans, LA, United States

\section{Background \& Purpose}

Brain death requires a step wise approach for accurate diagnosis. AAN Practice Perimeter recommends four ancillary tests to confirm brain death: nuclear medicine brain scintigraphy, transcranial doppler, electroencephalogram (EEG) and diagnostic angiogram. World Brain Death Project presented guidelines for determining brain death in adults, focusing on clinical exam. Due to the potential for false positive results, ancillary test should be use only as confirmation of brain death.

\section{Methods}

Case report

\section{Results}

A 40-year-old male with a past medical history of drug use was admitted after cardiac arrest at home. He was then cooled to 32-34 degrees $\mathrm{C}$ for neuroprotection. After rewarming, the patient was comatose without brainstem reflexes or over-breathing mechanical ventilation. Initial non-contrasted head CT showed diffuse loss of gray-white matter differentiation. MRI Brain without contrast demonstrated generalize cerebral edema with multifocal infarcts. Urine toxicology was positive for opiates; therefore, he did not meet prerequisite criteria for testing of brain death. Because of family discussions about confounders, dialysis was done to eliminate toxins. Urine toxicology was negative four days later. The patient demonstrated extensors posturing and did not met criteria for brain death. Neurological prognosis remained poor and ancillary testing was obtained for further family discussions. Ancillary testing showed isoelectric brain waves on electroencephalogram and absent brain blood flow on nuclear medicine brain scintigraphy. Patient was made DNR and expired.

\section{Conclusions}

Ancillary testing can lead to a false positive diagnosis of brain death and should only be used as confirmation. This case illustrates the importance of using them only in conjunction with the patient's clinical exam. 


\title{
Poster 67
}

\section{First Reported Case of Lactobacillus Rhamnosus Ventriculitis, Likely A Probiotic-induced Infection}

\author{
Sepideh Chagharvand, Sima Sayyahmelli, Mustafa Baskaya, Marin Darsie
}

University of Wisconsin Hospitals and Clinics, Neurology Department, Madison, WI, United States

\section{Background \& Purpose}

Probiotics are routinely administered to critically ill patients for many indications including improving GI health, and ventilator-associated pneumonia, however, potential concerns have been raised regarding the safety measurements that may outweigh their potential benefits. Lactobacillus bacteremia induced by probiotics has been well-reported in this high-risk population. However, to the best of our knowledge, there are two reports of Lactobacillus meningitis in immunosuppressed children.

\section{Methods}

N/A

\section{Results}

A 69-year-old woman presented with an aneurysmal subarachnoid hemorrhage secondary to a ruptured MCA aneurysm which was managed via clipping. Her 62-day hospital course was complicated by obstructive hydrocephalus requiring an EVD placement. On HD 30, her EVD was replaced in the operating room. The following day, a new fever prompted a CSF culture which grew Lactobacillus rhamnosus. Given the unusual organism, the daily Lactobacillus rhamnosus probiotic started on admission was the presumed source. She was treated with a 3-week course of antibiotics (meropenem narrowed to ampicillin). Ultimately, she required ventriculoperitoneal shunt placement and was discharged to an SNF with a mRS score of 4.

\section{Conclusions}

The efficacy and adverse effects of probiotic treatment in ICU patients have conflicting data in the literature. Despite the potential benefits of probiotics in critically ill patients, there are several case reports of systemic infections consistent with probiotic strains, including spontaneous bacterial peritonitis in patients with cirrhosis and on peritoneal dialysis, endocarditis, and bacterial meningitis. A risk-benefit analysis should be performed prior to the use of probiotics in this patient population, and probiotic-induced infections should be included in the differential for infection, particularly among highrisk patients with immunocompromised status, disorders of the gastrointestinal tract, or disrupted blood-brain barrier. Additional randomized controlled trials are necessary to evaluate the safety profile of probiotics in critically ill patients and can inform the optimal probiotic dose, species, formulation, and length of treatment. GI: Gastrointestinal, MCA: Middle cerebral artery, EVD: External ventricular drain, HD: Hospital day, CSF: Cerebral spinal fluid, SNF: Skilled nursing facility. mRS: Modified Rankin scale. 


\title{
Poster 68
}

\section{Fulminant Rhino-Orbital-Cerebral Mucormycosis With Carotid Invasion In Young Diabetic, An Argument For Aggressive Management.}

\author{
Eric Dornoff, Alisa Kanfi, Mario Zuccarello, Simona Ferioli \\ University of Cincinnati, Cincinnati, $\mathrm{OH}$, United States
}

\section{Background \& Purpose}

A 20-year-old male with a history of diabetes type I presented with dental pain, nose bleeding and emesis. In the Emergency Department he developed seizures, hemodynamic instability and respiratory failure. On arrival to the Neuro ICU neuro exam showed preserved mental status, left 6th nerve palsy, absent left corneal reflex. Work-up revealed serum leukocytosis and ketoacidosis. CSF showed WBC$559 / \mathrm{mcL}$, glucose $\mathrm{g} / \mathrm{dl}$, protein $-67 \mathrm{mg} / \mathrm{dl}$. MRI showed contrast enhancement in the frontal lobes and pansinusitis, concerning for angio-invasive sinusitis. He was started on IV antibiotics and antifungals. After a multidisciplinary discussion with ENT, neurosurgery, and neurocritical care, he underwent debridement of paranasal sinuses, left maxillectomy, left orbital exenteration, and tracheostomy. Pathology confirmed Rhizopus species. On day $5 \mathrm{MRI}$ brain showed new rim-enhancing fluid collections with mass effect, progression of cavernous sinus invasion and complete carotid occlusion without signs of cerebral ischemia. In light of these findings, neurosurgery performed a craniotomy to wash out the frontal lobe collections. Left carotid occlusion was confirmed in follow up CT angiograms.

\section{Methods \\ Case Report}

\section{Results}

Patient underwent reconstructive surgery 4 months after ICU discharge and was able to return to work.

\section{Conclusions}

Fulminant rhino-orbital-cerebral mucormycosis carries a poor prognosis with survival rates that have not improved over the last two decades. Carotid thrombus from fungal invasion, like seen in our case is a known ominous complication that could lead to ischemia and increase mortality. However, rapid initiation of liposomal amphotericin B and newer generation azoles, together with extensive surgical debridement are considered mainstay treatment and can improve survival and outcome. Our case highlights the importance of multidisciplinary approach and aggressive treatment to increase likelihood of survival, even in case of extensive cerebrovascular invasion. 


\title{
Poster 69
}

\section{Infiltrating Sphenoid Mass Post-COVID-19 Resulting in Pseudoaneurysm, Osseous Destruction of Cavernous Sinus and Multiple Ischemic Strokes}

\author{
Anish R. Patel \\ University of Southern California (Neurocritical Care), Los Angeles, CA, United States
}

\section{Background \& Purpose}

There has been emerging evidence showing that COVID-19 symptoms and consequences may still persist even after initial recovery. Reports of sinus destruction from infiltrating inflammatory and infectious sources raise a concern for secondary neurological injuries such as seizure and stroke.

\section{Methods}

Patient admitted to the neuro-ICU at a single tertiary care academic center one month after prolonged hospital admission for COVID-19 in January 2021 with stroke-like symptoms.

\section{Results}

61yo Hispanic male with PMHx of DM, HTN, recent CVA(no residual deficits), and COVID-19 4 months prior requiring 23 day hospital stay. Patient presented with slurred speech, right facial weakness, rightsided weakness with difficulty ambulating and CN-6 palsy. CTH/CTA notable for $1.7 \mathrm{~cm}$ pseudoaneurysm arising from cavernous segment of left ICA secondary to osseous destructive mass centered in sphenoid sinus with invasion of left cavernous ICA. MRI showed diffusion restriction near anterior choroidal region involving left thalamus and internal capsule. Patient underwent coil embolization of large left cavernous ICA pseudoaneurysm. Patient went for exploratory sphenoidotomy per ENT with no evidence of infection (no purulence) or invasive fungal sinusitis in the sphenoid. Edematous appearing mucosa was biopsied and sent for path, with no growth on micro and inflammatory cells noted on path. Patient stay complicated by leukocytosis and seizures. Lumbar puncture was unremarkable. Patient eventually received definitive treatment with pipeline stent and started on DAPT. Despite persistent right-sided deficits, seizures were controlled and he was discharged to a rehab facility.

\section{Conclusions}

There is limited evidence regarding lasting COVID-19 symptoms and secondary consequences after the initial infection is gone. However, there have been reports of individuals still experiencing symptoms and returning to the hospital even months after the initial infection. Patients should still be monitored closely after being discharged from the hospital through follow-up appointments and additional imaging may be warranted if new symptoms develop. 


\section{Poster 70}

\section{Insidious Presentation Of Brain Abscess: The Proof Is In The Pudding}

Emily Rogers, Amanda Tomlinson, Krishnan Ravindran, Jason Siegel

Mayo Clinic Florida, NeuroCritical Care, Jacksonville, FL, United States

\section{Background \& Purpose}

Purpose to describe an atypical presentation of cerebral abscess.

\section{Methods \\ Case report, literature review.}

\section{Results}

70-year-old female presented to outside hospital, with new onset seizure and 2 week progressive worsening headache and lethargy. Prehospital MRS 0 . Per family patient was characterized as a pathological hoarder with noted waste and pet feces throughout her home. Additionally ETOH abuse. Outside MRI demonstrated ring enhancing $L$ temporal lobe lesion with surrounding edema. Patient was informed it was suspicious for a malignancy then discharged on Keppra and Decadron. Family sought immediate additional evaluation through our institution's ER. Differential included possible neoplasm vs autoimmune vs infectious process. Laboratory data remarkable for leukopenia. LP demonstrated pleocytosis although cultures were negative. Empiric antibiotic coverage started due to suspicion of infectious abscess. Patient then underwent biopsy of L occipital lesion with intraoperative pathology concerning for abscess. Cultures remained negative. Progressive hydrocephalus with obtundation required intubation and R EVD placement. Due to noncommunicating hydrocephalus and inconsistent drainage of R EVD, the patient underwent $L$ frontal EVD placement. Frank malodorous white CSF drained immediately upon placement. Intraventricular antibiotics were added. Ultimately, R EVD CSF grew streptococcus pneumoniae, prevotella nigrescens, parvimonas micra, \& streptococcus anginosus along with positive parvovirus IGG. A third EVD was placed into $L$ temporal horn for decompression. Exam remained poor and the family withdrew medical support.

\section{Conclusions}

In setting of squalor and probable immunosuppression from chronic alcohol abuse, differential readily included infectious etiology of the lesion. However, initial CSF was bland and the patient did not demonstrate symptoms consistent with systemic or CNS infection (including fever, meningism). Repeated testing demonstrated a significant variation in infectious findings of CSF from alternate areas of sample. This emphasizes the significance of compromised CSF flow dynamics in the setting of brain abscess and ventriculitis. 


\section{Poster 71}

\section{Intra-arterial Thrombectomy after Cardiac Arrest in the thrombectomy Suite}

Rohan Mathur, Olanrewaju Agbe-Davies, Victor Urrutia, J. Ricardo Carhuapoma

Division of Neurocritical Care, The Johns Hopkins University School of Medicine, Baltimore, MD, United States

\section{Background \& Purpose}

Sparse evidence exists to support a delayed repeat attempt at thrombectomy after an unsuccessful first attempt due to medical deterioration. We describe the complex presentation of a woman with severe cardiac and renal comorbidities, who had a successful repeat thrombectomy with meaningful outcome after a cardiac arrest and renal failure.

\section{Methods}

Chart Review

\section{Results}

A 24-year-old woman with severe biventricular heart failure from a post-viral syndrome and CKD, presented with a LMCA syndrome, due to M1 occlusion (NIHSS 18, Aspects 8). Upon presentation, she had severe thrombocytopenia, and electrolyte derangements due to acute on chronic kidney injury. IV Alteplase was contraindicated. She was electively intubated and anesthetized for thrombectomy, prior to groin puncture, developed ventricular tachycardia followed by cardiac arrest. ROSC was 4 minutes. Vasopressors initiated for cardiogenic shock, and CVVHD was initiated. Thrombectomy was aborted. Overnight, electrolytes normalized, and vasopressor requirements decreased. 22 hours from her last known normal, MRI revealed a target-ADC core of $45 \mathrm{~mL}$ with complete left MCA territory hypoperfusion in the ASL sequence. She underwent thrombectomy based on DAWN criteria, with TICI$2 \mathrm{~b}$ reperfusion. Subsequent NCHCT showed no infarct growth. Neurological exam improved with only a mild expressive aphasia and mild right arm weakness at discharge. Five months later, per her wishes, she was transitioned to comfort care as her heart failure deteriorated. On follow up with her husband, he expressed gratitude for the few extra months of meaningful time with his wife.

\section{Conclusions}

The case highlights that critical care efforts, even after a severe initial presentation, can lead to a successful thrombectomy, and that eligibility for thrombectomy can be reassessed as the patient stabilizes. It also shows that in chronically ill patients with limited life expectancies, such efforts can offer enough quality time with loved ones to make it a meaningful outcome. 


\title{
Poster 72
}

\section{Intracerebral Hemorrhage and Double Inlet Left Ventricle. A Case Report and Review of The Literature.}

\author{
Hugo Gonzalez Gomez, Aaron Gusdon, Swathi Kondapalli
}

University of Texas Health Science Center at Houston, Department of Neurosurgery, Houston, TX, United States

\section{Background \& Purpose}

Double inlet left ventricle (DILV) is rare cardiac defect present in approximately $1 \%$ of all cases of congenital heart anomalies. Patients with unoperated DILV may survive into adulthood, with some patients living up to the 8th decade with medical management. Although ischemic strokes have been described in adult patients with Congenital Heart Disease (CHD), Intracerebral Hemorrhage (ICH) remains a rare phenomenon.

\section{Methods}

Herein we report a case of a patient with DILV presenting with ICH. We further reviewed the literature aiming to evaluate the current evidence and available guidelines.

\section{Results}

A 47 year-old Hispanic woman with history of CHD, hypertension, diabetes mellitus and prior ischemic strokes presented with acute encephalopathy. Initial Glasgow Coma Scale was 9. A non-contrast head CT showed a left temporoparietal ICH (120cc) with intraventricular extension, mass effect and multifocal cerebral herniation. ICH score was 3. CT Angiogram was negative for any vascular lesion. The patient underwent left-sided decompressive hemicraniectomy and hematoma evacuation. Refractory hypoxemia persisted despite maximal ventilatory support and inhaled nitric oxide. Echocardiogram revealed DILV, hypoplastic mitral valve, atrial septum defect with left to right shunt, and severe pulmonary valvular stenosis. Osmolar therapy with hypertonic saline was implemented to treat persistent mass effect and perihematomal edema. Development of persistent postoperative coagulopathy precluded invasive intracranial monitoring. Due to her poor prognosis, family decided to withdraw life support and the patient expired.

\section{Conclusions}

Based on our literature review, no specific guidelines are available to manage these patients and thus, evidence must be extrapolated from pediatric case reports and literature of other types of CHD, making management exceptionally challenging. It is unclear how the hemodynamic changes in DILV affect cerebral circulation and if invasive brain monitoring is indicated. Further studies are needed to understand the pathophysiology of the DILV in ICH to develop evidence-based guidelines. 


\title{
Poster 73
}

\section{Intrathecal Methotrexate Toxicity Resulting in Brain Death due to Generalized Cerebral Edema: A Case Report}

\author{
Haoming Pang, Maranatha Ayodele, Julie L. Chan, PhD, Michael Gezalian, Shouri Lahiri, Shahed Toossi \\ Cedars-Sinai Medical Center, Departments of Neurology and Neurosurgery, Los Angeles, CA, United \\ States
}

\begin{abstract}
Background \& Purpose
Intrathecal (IT) methotrexate (MTX) is used to treat central nervous system malignancies. While adverse events such as acute and subacute encephalopathy, headaches, and aseptic meningitis may occur, severe adverse events including life-threatening cerebral edema are not well described. We describe such a case to further understand the lesser known complications of IT MTX.
\end{abstract}

\section{Methods}

Data collected via retrospective chart review.

\section{Results}

A 37-year-old BRCA1+ woman with metastatic triple-negative breast cancer status post mastectomy and palliative chemotherapy presented with two weeks of confusion. MRI demonstrated widespread leptomeningeal enhancement along the right temporal cortex, insula, and cerebellum suggestive of leptomeningeal carcinomatosis. Cerebral spinal fluid cytology confirmed the presence of malignant cells. Patient underwent placement of an Ommaya reservoir with subsequent intra-operative administration of $12 \mathrm{mg}$ of IT MTX. Postoperative head computed tomography (CT) revealed good placement of the Ommaya reservoir. Twelve hours postoperatively, she became agitated. Sixteen hours postoperatively she became increasingly lethargic and ultimately unresponsive. Eighteen hours postoperatively she developed bilateral $5 \mathrm{~mm}$ non-reactive pupils and loss of all brainstem reflexes. Notably, she never experienced cardiopulmonary arrest or prolonged hypoxia. Subsequent head CT 19 hours after the initial postoperative imaging revealed diffuse cerebral edema causing uncal and tonsillar herniation. MRI revealed similar findings. Post-mortem autopsy demonstrated an edematous brain consistent with severe cerebral edema and deep parenchymal cancerous deposits within the Virchow-robin spaces and leptomeninges. White matter showed no demyelination or leukoencephalopathy.

\section{Conclusions}

Cerebral edema is a rare but potentially devastating neurological complication following IT MTX for leptomeningeal carcinomatosis. In our literature review, our patient is among those who developed the most severe and rapid neurologic deterioration, resulting in diffuse cerebral edema leading to death. The possibility of such a severe adverse event should be considered when weighing the risks and benefits of IT MTX in the treatment of leptomeningeal carcinomatosis. 


\section{Poster 74}

\section{Kilauea Sign: Paradoxical Bifrontal Cerebral Ischemic Syndrome after Direct Revascularization Surgery for Moyamoya Disease}

Kazuma Nakagawa, Matthew A. Koenig, Elaine Lau, Stacy Brown, Chung-Huan Sun, David Panczykowski

The Queen's Medical Center, Neuroscience Institute, Honolulu, HI, United States

\section{Background \& Purpose}

Ischemic complications following direct revascularization surgery for Moyamoya Disease (MMD) may occur despite immediate cerebral blood flow augmentation to the ipsilateral hemisphere. We report a case series of paradoxical bifrontal cerebral ischemic syndrome ("Kilauea sign") among MMD patients who had undergone successful direct revascularization (STA-MCA bypass) surgery.

\section{Methods}

All MMD patients who underwent direct revascularization surgery between October 1, 2019 and November 1, 2020 were retrospectively reviewed. All patients underwent CT or MR angiography and perfusion studies immediately postoperatively per institutional pro

\section{Results}

Among a total of 21 cases of direct bypass surgeries (19 patients), bifrontal cerebral hypoperfusion syndrome (Kilauea sign) were observed in 6 cases (4 patients) postoperatively despite post-operative CT angiography demonstrating patent grafts. The two patients who initially demonstrated the Kilauea sign developed a similar radiographic pattern after their subsequent contralateral bypass surgery. All 6 cases with the Kilauea sign, compared to only 3 of the 15 cases without the Kilauea sign, had underlying bilateral severe anterior cerebral artery (ACA) stenoses ( $100 \%$ vs. $20 \%$ respectively, $\mathrm{P}<0.001)$.

\section{Conclusions}

Paradoxical bifrontal cerebral ischemic syndrome can occur after direct revascularization surgery for Moyamoya Disease and may be associated with pre-existing bilateral severe ACA stenoses. 


\section{Poster 75}

\section{Lidocaine Infusion as an Effective Means of Pain Management}

Tse Chiang Chen, Olivia Cornu, Julio E. Vega, Ali Shirazian, Kareem Elzamly, Shannon Hextrum, Aimee Aysenne

Tulane University Medical Center, Department of Neurosciences, New Orleans, LA, United States

\section{Background \& Purpose}

Pain management for patients in the neurocritical care unit poses several challenges. One must balance adequate analgesia while minimizing over sedation impeding neurological assessment and depressing respiratory drive and avoiding analgesia dependence. Previous studies suggest IV lidocaine relieves various types of pain including post spinal surgery, diabetic neuropathy, post-stroke, spinal cord injuries. We present a case series of patients treated with lidocaine infusion in the neurocritical care setting.

\section{Methods}

Our lidocaine infusion protocol uses an initial bolus of $0.3 \mathrm{mg} / \mathrm{kg}$ followed by a basal infusion at $1 \mathrm{mg} / \mathrm{kg} / \mathrm{hr}$ using ideal body weight for 3 days. Patients were monitored with daily EKGs. Some patients had concurrent pain medications in either scheduled or a

\section{Results}

Eleven of 14 (79\%) were major spine surgery patients, all of whom also had prior and concurrent opiate regimen. Four (29\%) major spine patients had a history of IVDU. Three patients $(21 \%)$ had other diagnoses: 1 (7\%) hemiplegic migraine, $1(7 \%)$ herpetic occipital neuralgia , $1(7 \%)$ intracranial hemorrhage. Of these, one was on an opiate prior to starting lidocaine infusion, and none of them were on concurrent opiate medications. There were no complications in any patient. Thirteen of 14 (92\%) patients reported subjective improvement of pain.

\section{Conclusions}

IV Lidocaine is safe and effective for pain control in the Neuro ICU, as an opiate adjunct or alone. Further investigations for expanded uses are encouraged. 


\section{Poster 76}

\section{Management of Refractory Bacterial Meningitis-Associated Cerebral Vasospasm: A Case Report}

Sofya Norman, Jon Rosenberg, Sri Hari Sundararajan, Srikanth Reddy Boddu, Judy H. Ch'ang

Weill Cornell Medical College, New York, NY, United States

\section{Background \& Purpose}

Cerebral vasospasm is a dangerous complication of bacterial meningitis and a significant indicator of poor prognosis. There is a paucity of literature on management of infectious vasospasm. We report on a case of severe refractory meningitis-associated vasospasm that was ultimately responsive to intravenous (IV) and intra-arterial (IA) milrinone, and balloon angioplasty.

\section{Methods}

Data was collected through chart review.

\section{Results}

A 53-year-old male presented for craniopharyngioma resection. Post-operatively he developed an iatrogenic klebsiella oxytoca meningitis with CSF leukocytosis $3300 / \mathrm{uL}$, glucose $<4 \mathrm{mg} / \mathrm{dL}$, and protein > $500 \mathrm{mg} / \mathrm{dL}$. Despite treatment with steroids and antibiotics, his course was further complicated by infectious vasospasm of the bilateral internal carotid arteries, middle cerebral arteries, anterior cerebral arteries, and posterior cerebral arteries seen on transcranial doppler (TCD) and CT angiography (CTA). The patient was treated with induced hypertension and IA verapamil, but his neurologic exam continued to worsen, developing new left gaze deviation and left hemiplegia. TCDs/CTA continued to show diffuse vasospasm, and MRI revealed a left pontine and right internal capsule stroke. Vasospasm continued to remain refractory to induced hypertension and IA verapamil. Therefore, the patient was started on IV milrinone (up to $75 \mathrm{mcg} / \mathrm{kg} / \mathrm{hr}$ ) with improvement of neurologic exam, followed by IA milrinone (6mg) and balloon catheter angioplasty. Following these interventions, the patient demonstrated improvement in radiographic vasospasm in addition to his left hemiplegia and arousal.

\section{Conclusions}

This case supports the use of milrinone and balloon angioplasty as treatments for severe refractory infectious vasospasm. Prior studies have shown that IV and IA milrinone can be used to treat vasospasm secondary to aneurysmal subarachnoid hemorrhage. In future cases of infectious vasospasm, IV and IA milrinone should be trialed earlier with consideration of angioplasty. To our knowledge, this is the first report of using IV and IA milrinone to treat infectious vasospasm. 


\title{
Poster 77
}

\section{Management of Tandem Occlusions in Patients who Receive rtPA: Initial Results of a CT Perfusion Protocol}

Keaton S. Smetana, Amanda Zakeri, Allison Huttinger, Patrick Youssef, Casey C. May, Shahid M. Nimjee

\author{
The Ohio State University Wexner Medical Center, Columbus, OH, United States
}

\section{Background \& Purpose}

Tandem occlusions exist in 17-32\% of large vessel occlusion (LVO) strokes. When emergent carotid stenting is performed in tandem with mechanical thrombectomy (MT), bleeding risk is of high concern due to administration of intravenous antiplatelet agents after receipt of tPA. Dosing specifics of antithrombotics nor the utility of CT perfusion imaging are clear in previous literature.

\section{Methods}

We report preliminary observational data of using eptifibatide for emergent stenting of an ipsilateral cervical ICA lesion with $>70 \%$ stenosis after tPA and MT for tandem occlusions. We propose a preliminary protocol using CT perfusion to guide management.

\section{Results}

Four males, 63 years old [IQR 55.8-73.3], with a baseline NIHSS of 12 [IQR $7-18$ ] were included. The RAPID CTP ${ }^{\circledR}$ found the median core volume was $9.5 \mathrm{~mL}$ [IQR $\left.6-18.3\right]$, penumbra volume $109 \mathrm{~mL}$ [63.5 151.3], and a mismatch ratio of 13.2 [IQR 7.5 - 17.3]. The ASPECTS score was 9.5 [IQR 8.5 - 10]. The time from symptom onset to tPA bolus was 2.4 hours [IQR 2.2-2.7], and tPA bolus to reperfusion was 3.3 hours [IQR 2.8 - 3.6]. All cases had a TICl score of $2 \mathrm{~b} / 3$. The time from tPA bolus to eptifibatide bolus $(90 \mathrm{mcg} / \mathrm{kg}$ ) was 3.7 hours (IQR $3.2-4)$ and the continuous infusion $(0.25 \mathrm{mcg} / \mathrm{kg} / \mathrm{min})$ duration was 11.7 hours (IQR $11.2-12.2$ ). Median times from tPA bolus to aspirin and clopidogrel were 11.8 hours [IQR $9.5-15$ ] and 12.8 hours [IQR 11 - 15] respectively. The median length of stay was 5.5 days (IQR $4.5-6$ ). No patients developed symptomatic ICH nor did they have post-op bleeding from the access site.

\section{Conclusions}

Four patients had successful placement of emergent stents with tandem lesions using CT perfusion data, based on DEFUSE-3, with standardized eptifibatide dosing. These findings should be interpreted cautiously, and need to be confirmed in a larger patient population. 


\title{
Poster 78
}

\section{Mechanical Thrombectomy in Acute Ischemic Stroke during Pregnancy has Favorable Outcome: Case Report to Open Further Discussion}

\author{
Maria Shoaib; Aruna Paul, Zainab Al Obaidi, Blair Apple, Chao Xu, Evgeny Sidorov
}

University of Oklahoma Health Sciences Center, Department of Neurology, Oklahoma City, OK, United States

\section{Background \& Purpose}

The relative risk of cerebral infarction of 0.7 during pregnancy which rises to 5.4 in the 6 weeks following delivery. The objective of this case report is to discuss the safety of mechanical thrombectomy in large vessel occlusion stroke in pregnancy and bridge the professional practice gap as there is limited knowledge regarding the benefit and safety of intervention amongst pregnant and post-partum population given it was an exclusion criterion in endovascular thrombectomy (EVT) trials.

\section{Methods}

Retrospective chart review of stroke/endovascular database from 2013-2020 at the University of Oklahoma health sciences center and we found 1 case of mechanical thrombectomy in a pregnant woman.

\section{Results}

28-year-old, woman in her 13th week of pregnancy with a history of Still disease and Rheumatoid Arthritis (diagnosed at age of 4, S/P bilateral hip replacement, on Prednisone 20mg daily), smoking, and substance use disorder with methamphetamine who was admitted for acute ischemic stroke in the left MCA territory with left distal M1 clot secondary to hypercoagulable state (given young age, current pregnancy and history of miscarriages in the past). NIHSS 13, mRS 0. No IV tPA (outside the window). CT perfusion images suggested a moderate-sized penumbra involving most of the MCA territory, she was taken for mechanical thrombectomy with $\mathrm{TICl} \mathrm{Ilb.} \mathrm{Post} \mathrm{thrombectomy} \mathrm{patient} \mathrm{was} \mathrm{started} \mathrm{on} \mathrm{a} \mathrm{heparin}$ drip and was switched to Lovenox for secondary stroke prevention and discharged to rehab with an unremarkable hospital course. Her stroke symptoms of right hemiparesis \& aphasia resolved in 3-4 months and she was back to her baseline functional status. She opted for an elective C-Section at the 37 th week of her pregnancy and had a healthy pregnancy outcome.

\section{Conclusions}

We observed that in our case report pregnant women had similarly favorable outcomes as to nonpregnant women. We recommend future multi-center studies to identify the hospital outcomes of pregnant and postpartum ischemic stroke patients with large vessel occlusion who are most likely to safely benefit from mechanical thrombectomy. 


\section{Poster 79}

\section{Medical Student \& Resident Education in the NeurolCU}

Charles M. Andrews, Danuel K. Snelgrove

Medical University of South Carolina, Department of Neurosurgery, Charleston, SC, United States

\section{Background \& Purpose}

Most curriculum developed for the NeuroICU has focused on fellowship training and APP onboarding. Despite this, many students and residents rotate through the NeurolCU with a poorly designed curriculum, objectives, and orientation to the unit. This often is true for students from other disciplines including pharmacy and APP students. Most clinical student learning focuses on observational clinical care and didactics. Modern students and residents often require and benefit from alternative learning methods and means.

\section{Methods}

The authors reviewed the previously developed NeurolCU Handbook, any orientation materials and how students and residents are learning in the ICU environment.

\section{Results}

We modified the previous version of the handbook that was composed of several neurocritical care articles written for different levels of training and compiled a digital version of updated 'core curriculum' content with all reading and materials built within the document. Providing overview summaries of core content, or "one pagers" with additional reading allows learners to grasp key concepts then read into the background. This digital handbook was then placed on a newly created NeurolCU Education website that was created by the authors with additional content of expectations, orientation and objectives, schedules and links to additional educational content. The authors have additionally begun to record short lectures on the core content that learners can view from the site that reinforce key concepts in neurocritical care. In addition to creation of curriculum and content, the authors have created pre and post course assessments to ensure understanding of these concepts.

\section{Conclusions}

Student and resident education should be more developed and enhanced with clear curriculum, objectives and content. NCS members may be able to help identify core themes and content that early learners should be able to identify. 


\title{
Poster 80
}

\section{Minimally Invasive Evacuation of Spontaneous Intracerebral Hemorrhage Using a Tubular Retractor System in the Setting of CTA Spot Sign, a Case Series}

\author{
Joseph Falcone, Jefferson Chen \\ University of California, Irvine, Orange, CA, United States
}

\section{Background \& Purpose}

Here we demonstrate use of a tubular retractor system for early evacuation of spontaneous supratentorial intracerebral hemorrhage $(\mathrm{sICH})$ in a series of patients with spot sign on computed tomography angiography (CTA), which has previously been seen as a contraindication for minimally invasive surgical techniques.

\section{Methods}

This is a retrospective review of patients with $\mathrm{SICH}$ presenting to the University of California Irvine Medical Center with spot sign on CTA, from 2018-2020, who were treated with a minimally invasive perifascicular approach for sICH evacuation using a tub

\section{Results}

9 patients ( 7 men and 2 women) with a mean age of 56.8 years were included in this study. There was significant decrease between pre-operative and post-operative ICH volumes $(68.32 \mathrm{~cm} 3 \pm 37.04$, vs $15.94 \mathrm{~cm} 3 \pm 16.94, p=0.0014)$ as well as ICH score $(2.29 \pm 0.9$, vs $1.7 \pm 0.9, p=0.011)$. Mean time from arrival to surgery was 10.90 hours ( \pm 14.30 hours), and in 7 patients was less than 8 hours. Mean percentage of hematoma evacuation was $77.67 \%$ ( $\pm 19.88 \%)$. There were no complications related to the surgery in any of the cases, with no uncontrollable intraoperative bleeding and no pathology demonstrating occult vascular lesion. In-hospital mortality was $11.11 \%$ and mean Modified Rankin Score at discharge was $4.6( \pm 1.3)$.

\section{Conclusions}

Stroke in the form of sICH is associated with high morbidity and mortality, and the impact of surgery on functional outcomes remains an active area of investigation. Early intervention with a tubular retractor for a minimally invasive perifascicular approach appears to be a safe and effective means of hematoma evacuation despite the presence of CTA spot sign, and this finding should not delay early intervention when indicated. Achieving intraoperative hemostasis may be easier with the direct visualization provided by a tubular system compared to other minimally invasive techniques. 


\title{
Poster 81
}

\section{Neurological Impact of Hyperammonemia Induced by Ureaplasma Parvum Infection Post Lung Transplantation - A Case Series}

\author{
Jan Bittar, Blake Senay, Bryan Gough, Shraddha Mainali
}

The Ohio State University Wexner Medical Center, Department of Neurology, Columbus, OH, United States

\begin{abstract}
Background \& Purpose
Hyperammonemia is a rare but often fatal complication following lung transplantation. Opportunistic bacterial infections by Ureaplasma and Mycoplasma species have been identified as contributors to this metabolic disturbance. Hyperammonemia has been well recognized as a cause of encephalopathy and seizures due to the neurotoxic effect. Here we highlight the neurological impact of hyperammonemia from Ureaplasma parvum infection following bilateral lung transplant.
\end{abstract}

\section{Methods}

Retrospective chart review of two cases.

\section{Results}

Case 1: A 69-year-old male presented for bilateral lung transplant due to idiopathic pulmonary fibrosis. Hospital course was complicated by new onset super-refractory status epilepticus (SRSE) secondary to hypeammonemia (412 umol/L) of unclear etiology. Electroencephalogram (EEG) revealed multifocal epileptogenicity and brain magnetic resonance imaging (MRI) was suggestive of hyperammonemic encephalopathy. Infectious workup revealed Ureaplasma parvum in bronchoalveolar lavage (BAL). Patient was started on Doxycycline and Levofloxacin. Although use of pharmacologic agents and continuous renal replacement therapy (CRRT) helped lower ammonia level, patient failed to wean off high dose Ketamine drip for SRSE and ultimately succumbed to his disease.Case 2:A 63- year-old male presented for bilateral lung transplant due to acute respiratory distress syndrome following Coronavirus Disease 2019 (COVID-19) pneumonia. A few days after surgery, patient developed acute encephalopathy with abnormal movements of the head and neck. Lab workup revealed hyperammonemia ( $189 \mathrm{umol} / \mathrm{L}$ ) of unknown etiology. EEG and brain MRI were unremarkable. Infectious workup revealed Ureaplasma parvum in BAL. Patient was started on Doxycycline and Levofloxacin along with CRRT. His ammonia level improved along with improvement in mental status and resolution of involuntary head and neck movements.

\section{Conclusions}

Unexplained hyperammonemia in post-transplant patients should raise the concern for underlying Ureaplasma or Mycoplasma infection as a possible etiology. Aggressive treatment strategies to lower serum ammonia and bacterial source control may help prevent irreversible brain injury with poor neurologic outcome. 


\title{
Poster 82
}

\section{New Onset Refractory Status Epilepticus, associated with COVID19, and treated with Tocilizumab}

Rohan Mathur, Karissa Arthur, Clotilde Balucani, Kathryn Rosenblatt, Khalil Husari, Emily Johnson, John

\author{
C. Probasco, Jose I. Suarez
}

Division of Neurocritical Care, The Johns Hopkins University School of Medicine, Baltimore, United States

\section{Background \& Purpose}

New Onset Refractory Status Epilepticus (NORSE) is a rare and severe presentation in patients without a pre-existing history of seizures. We describe a case associated with a COVID19 infection that resolved with Tocilizumab therapy.

\section{Methods}

Chart Review, Literature Review

\section{Results}

A 40-year-old woman, otherwise healthy, developed new seizures and presented to an outside hospital. She was found to be COVID-19 positive with bilateral lung infiltrates and admitted. Over two weeks, she developed progressive confusion and hearing loss, prompting transfer to our center. Continuous EEG demonstrated increasing frequency of focal seizures with secondary generalization that progressed into non-convulsive status epilepticus. Multiple anti-seizure medications were loaded without effect. She was intubated and started on a midazolam infusion, and subsequently ketamine and pentobarbital infusions for burst suppression. MRI Brain showed bilateral T2-FLAIR hyperintensities. CSF analysis was unrevealing; her autoimmune panel showed low seropositivity for Anti-GAD65. Multiple attempts to wean midazolam and pentobarbital were unsuccessful due to breakthrough status epilepticus. Brain biopsy was unrevealing and notably showed no lymphocytic infiltration. Ketogenic Diet, and courses of steroids and plasmapheresis were not efficacious. Tocilizumab was administered with significant improvement in cEEG over 48-72 hours. Midazolam and pentobarbital were then successfully weaned and discontinued. She remained comatose for another 3 months, with her course complicated by a severe critical illness neuropathy. She then emerged from coma, and is now following commands and communicating basic needs

\section{Conclusions}

NORSE is a severe clinical phenomenon and cases associated with COVID19 are exceedingly rare. Tocilizumab, a humanized monoclonal antibody against the IL- 6 receptor, has been used successfully in case studies for the management of NORSE, and recently for severe COVID19 pneumonia. We describe a rare case of NORSE associated with COVID19, with extensive workup, and highlight the possible beneficial effect of Tocilizumab, and the extended time frame for neurological recovery. 


\title{
Poster 83
}

\section{Opiates can Lower Ticagrelor P2Y12 Activity in Neuroendovascular ICU Patients}

\author{
W. David Freeman, Krina Vyas, Ashley Rogers, Christopher Kyper, Rabih Tawk
}

Mayo Clinic Neurosciences Intensive Care Unit, Jacksonville, FL, United States

\begin{abstract}
Background \& Purpose
A recent discovery of drug-drug interactions between opioids and P2Y12 inhibitors may have clinically relevant consequences for patients undergoing neuroendovascular procedures. Opioids are well-known to decrease gastrointestinal motility and lead to delayed absorption of oral medications. Several randomized trials have shown decreased absorption of P2Y12 inhibitors when used concurrently with opioids. However, mixed data exists regarding its clinical effect. In this case report, we describe a case of diminished ticagrelor activity when used concurrently with opioids.
\end{abstract}

\section{Methods}

Case report of a patient with acute middle cerebral artery (MCA) intra-stent thrombosis despite aspirin and ticagrelor therapy.

\section{Results}

A 56-year-old male underwent elective stent-assisted coiling of right posterior communicating artery aneurysm with stent migration into proximal MCA. Post-procedure, the patient's NIH stroke scale was 19 and brain attack was called. CT angiogram and perfusion showed a perfusion deficit of right MCA territory. Therefore, digital subtraction angiogram performed confirming intra-stent thrombosis of right MCA with successful thrombectomy and intra-arterial tirofiban followed by aspirin and ticagrelor load. During hospitalization, the patient's platelet function was closely monitored via P2Y12 assay. The day of admission, platelet assay value was low at 12. During hospitalization, the patient received boluses of fentanyl for pain averaging at $175 \mathrm{mcg}$ per day. On subsequent days, the patient's platelet assay was 167, then 203. Neurocritical care team decreased opiate dosing with platelet assay then 151 . The aspirin reaction unit remained in therapeutic range throughout hospitalization. The patient takes oxycodoneacetaminophen for chronic pain management as an outpatient.

\section{Conclusions}

Long term use of oxycodone and high doses of fentanyl during hospitalization reduced the efficacy of ticagrelor. Lower drug concentration and reduced platelet activity of ticagrelor was noted from coadministration with opiates. This case describes clinical consequences of such drug-drug interactions and possible avoidance of co-administration of these agents. 


\title{
Poster 84
}

\section{Pain control and anxiolysis after subarachnoid hemorrhage using immersive virtual reality: A case report and preliminary results from ongoing single center trial}

\author{
Adam Kardon, Robert S. Murray, Mazhar Khalid, Luana Colloca, J. Marc. Simard, Neeraj Badjatia, Sarah \\ B. Murthi, Nicholas A. Morris
}

University of Maryland, Department of Neurology, Baltimore, MD, United States

\begin{abstract}
Background \& Purpose
Debilitating headache persists after acute subarachnoid hemorrhage (SAH). Despite high prevalence, little is known regarding optimal treatment strategies. Nonpharmacologic adjunctive therapies are emerging as tools to help treat pain and limit opioid exposure. Virtual reality (VR) is an immersive audiovisual experience that has been shown to reduce pain perception in other patient populations, however the role of VR in acute brain injury is unknown. Here, we describe a clinical case in which VR was effective in reducing headache after $\mathrm{SAH}$. In addition, we report preliminary results from an ongoing, prospective trial utilizing VR for headache in patients with SAH.
\end{abstract}

\section{Methods}

We report the case of a 45-year-old female with aneurysmal SAH who suffered from persistent headaches during her hospitalization despite escalation of analgesic pharmacotherapy. A trial of VR was used as an adjunct to medication, and symptoms were assesse

\section{Results}

The patient in our case report used VR daily over a four-day period. After each session, she reported a decrease in both pain and anxiety. Overall, her pain scores improved during VR therapy, lasting several days after the last VR session before rebounding to pre-VR levels. Interim analysis of ten patients in a prospective trial of VR in SAH-related headache demonstrated a trend towards improved neck pain in patients undergoing VR versus a $2 \mathrm{D}$ control scenario $(p=0.07)$.

\section{Conclusions}

VR may provide additional analgesia and anxiolysis over pharmacologic measures alone and warrants further study in patients with SAH. 


\section{Poster 85}

\section{Posterior Reversible Encephalopathy Syndrome (PRES) from the Combined use of Intravenous, Intra- Arterial, and Intrathecal Vasodilating Agents and Modest Blood Pressure Augmentation in the Treatment of Vasospasm in Subarachnoid Hemorrhage}

Baback Arshi; Kiddy L. Ume, Kevin Chen, Elise Rippelmeyer

Washington University in St. Louis, Department of Neurology, Saint Louis, MO, United States

\section{Background \& Purpose}

Posterior reversible encephalopathy syndrome (PRES) is a reported complication of blood pressure augmentation for cerebral vasospasm in aneurysmal subarachnoid hemorrhage (aSAH). Besides IA verapamil, IV Milrinone and IT Nicardipine have recently found roles in the treatment of refractory vasospasm. The complications arising from the combined use of blood pressure augmentation and vasodilating agents have not been widely studied.

\section{Methods}

We report a case of PRES developing with the combined use of IV milrinone, IT Nicardipine, and IA verapamil and modest blood pressure augmentation.

\section{Results}

A 62-year-old woman presented with a Hunt-Hess grade 5, modified Fisher grade 4 aSAH and a right convexity subdural hematoma (SDH). Cerebral angiography demonstrated an $8.8 \times 8.0 \mathrm{~mm}$ anterior communicating segment aneurysm, which was endovascularly coiled. She underwent right craniotomy for SDH evacuation. She was clinically improving and extubated on day 4. Her initial repeat angiogram was negative for vasospasm. Her hospital course was complicated by encephalopathy, fluctuating leftsided weakness and neglect, and worsening respiratory status from COPD exacerbation, for which she was re-intubated. Subsequent angiograms showed persistent bilateral anterior cerebral artery vasospasm, and she was treated multiple times endovascularly with IA verapamil, IA milrinone, and angioplasty. An external ventricular drain was placed for IT nicardipine on day 9. Continuous EEG was negative for seizures. Her clinical status did not improve despite blood pressure augmentation with MAP above $100 \mathrm{mmHg}$ and IT nicardipine. A milrinone infusion was added on day 10 without appreciable improvement. A brain MRI on day 13 showed bilateral FLAIR hyperintensities consistent with PRES.

\section{Conclusions}

The combination IV milrinone and blood pressure augmentation with vasopressors in SAH patients may predispose to cerebral vasodilation and capillary leakage which may precipitate PRES occurring at lower blood pressure targets. Studies to investigate the cardiopulmonary and intracranial complications of such combined regimen are warranted. 


\title{
Poster 86
}

\section{Posterior Reversible Encephalopathy Syndrome Related to High Does Corticosteroid Treatment for Idiopathic Thrombocytopenic Purpura}

\author{
Weizhe Li, Jennifer Glover, Keith E. Dombrowski
}

University of South Florida, Department of Neurology, Tampa, FL, United States

\section{Background \& Purpose}

Posterior reversible encephalopathy syndrome (PRES) is an acute neurological complication characterized by seizures, focal neurological deficit, and radiological findings of vasogenic edema in the parietal and occipital white matters. Although steroids have been linked to occurrence of PRES, its role as a precipitant remains undetermined. We hereby report a case of patient with intracranial hemorrhage in the context of idiopathic thrombocytopenic purpura (ITP) who was treatment with high dose steroid developed PRES.

\section{Methods}

This case was reported from an academic medical center. Patient developed PRES after receiving high dose steroid therapy for ITP.

\section{Results}

A woman in late 40s developed severe headache and left hemiparesis one week after a hysterectomy for multiple uterine fibroids. Imaging studies showed large right frontal intraparenchymal and subarachnoid hemorrhage. She underwent an emergent right frontal craniotomy for hematoma evacuation. Perioperative labs revealed thrombocytopenia with platelet count of $41,000 / u l$, hemoglobin of $10.7 \mathrm{~g} / \mathrm{dL}$. Her platelet count failed to rise after transfusion of 5 units of platelets. A primary autoimmune-mediated thrombocytopenia was suspected after exclusion of heparin induced thrombocytopenia and microangiopathic hemolytic anemia. High dose dexamethasone 40mg daily for presumed ITP was started. Although her platelet count increased rapidly to 292,000/ul in 3 days, she developed a sudden decline in consciousness with focal motor seizure activity. Magnetic resonance imaging finding of T-2 hyperintensity in posterior occipital lobes, together with bilateral posterior cerebral artery irregularities on cerebral angiography, favored a diagnosis of PRES. Careful review of her clinical course and medication list failed to identify common culprits. Steroids was weaned off, her mental status and imaging improved correspondingly.

\section{Conclusions}

Awareness of potential association between PRES and high dose steroid treatment is important. Extra vigilance should be practiced when utilizing high dose steroid in patient with other precipitating factors for PRES. 


\title{
Poster 87
}

\section{Pulmonary Complications after Neurosurgery in a TBI Patient with COVID-19: A Case Report}

Charlotte Zhong, Christopher D. Nguyen, Roy A. Poblete

\author{
University of Southern California, Keck School of Medicine Department of Neurology, Los Angeles, CA, \\ United States
}

\section{Background \& Purpose}

Accumulating evidence suggests that elevated inflammatory markers predict the clinical severity of COVID-19 infection, but it is unknown whether proinflammatory states such as trauma or surgery can activate infection in asymptomatic COVID-19 patients. Studies have shown that postoperative pulmonary complications can occur in up to half of patients with perioperative COVID-19 infection. Our case illustrates the potential roles traumatic brain injury (TBI) and neurosurgery can have in triggering symptomatic COVID-19 infection and highlights the unique challenges in managing pulmonary complications in patients with TBI.

\section{Methods}

A chart and literature review of a case.

\section{Results}

A 30-year-old male with severe TBI is incidentally found to be COVID-19 positive on admission. Initial trauma imaging of the thorax demonstrated minimal airspace disease. The patient's intracranial pressures were refractory to medical management thus an emergent hemicraniectomy was performed. Postoperatively, he developed acute respiratory distress syndrome that required prone positioning. One week later, the patient expired unexpectedly after sudden cardiac arrest, presumed to be secondary to a large pulmonary embolism.

\section{Conclusions}

Undergoing emergency neurosurgery may lead to activation of an otherwise indolent COVID-19 infection in patients with TBI. Management of COVID-19 ARDS in patients with a concomitant acute brain injury can be particularly challenging since TBI standards of care can come into conflict with what is considered best practice in terms of corticosteroid use, anticoagulation, fluid balance, and ventilator management. 


\title{
Poster 88
}

\section{Rapid Brain Death Caused by Brain Abscess Associated with Over-the-Counter Sinus Irrigation}

Franziska Herpich, Eunice Lee, Muhammad K. Athar

\author{
Thomas Jefferson University Hospital, Department of Neurology, Division Neuro critical Care, \\ Philadelphia, PA, United States
}

\section{Background \& Purpose}

Over-the-counter sinus irrigation devices (neti pots) are commonly recommended by doctors to treat sinus congestion and are deemed overall relatively safe. Given the widespread use of these devices, it is important to report possible associated adverse events, in this case a brain abscess which progressed rapidly to brain death.

\section{Methods}

Via Chart review

\section{Results}

43-year-old man with mild confusion for 4 weeks followed by progressive somnolence. Recommended by his family physician, he recently started using a neti pot for chronic sinusitis. On the day of admission, he was brought in by family after a witnessed seizure. In the emergency department (ED), the patient was initially awake, yet confused with perseveration of speech. After another witnessed seizure, he was intubated and loaded with Levetiracetam. The CT-head showed a right frontal $4.1 \mathrm{~cm}$ necrotic mass extending into the corpus callosum with surrounding vasogenic edema and $7 \mathrm{~mm}$ midline shift without evidence of herniation. Post intubation his exam remained poor, however brain stem reflexes were intact. He was transferred to the neurointensive care unit where upon initial evaluation he had absent brainstem reflexes and no motor response. Stat repeat CT head showed progression in effacement of lateral ventricles with uncal herniation. An emergent external ventricular drain was placed with opening pressure of $30 \mathrm{cmH} 2 \mathrm{O}$. Despite good placement confirmed on imaging, no ICP waveform was transducable. CSF studies showed significant pleocytosis and CSF cultures grew Streptococcus intermedius while blood cultures were positive for Parvimonas micra. Despite hyperosmolar therapy, EVD and hyperventilation, the patient's exam did not improve over the next 24 hours and he was declared brain dead.

\section{Conclusions}

In the absence of other risk factors, it is possible that aggressive irrigation of colonized sinus mucosa might lead to spreading of infection. Further data is required to establish if neti pots are truly a risk factor for brain abscesses. 


\title{
Poster 89
}

\section{Rapidly Progressive Diffuse Cerebral Edema with Status Epilepticus One Month after Chimeric Antigen Receptor T cell (CAR-T) Therapy}

\author{
Rajat Dhar, Kiddy L. Ume
}

Washington University in St. Louis, School of Medicine, Dept. of Neurology, St. Louis, MO, United States

\section{Background \& Purpose}

Immune effector Cell-Associated Neurotoxicity Syndrome (ICANS) is a complication of therapies involving activation of endogenous or infused immune effector cells and is most commonly seen after chimeric antigen receptor T-cell (CAR-T) therapy. It typically presents with encephalopathy, focal signs, and/or seizures shortly after chimeric antigen receptor T cell (CAR-T) therapy. Delayed neurotoxicity causing rapidly progressive cerebral edema has not been reported.

\section{Methods}

We report a case of rapidly progressive diffuse cerebral edema presenting with myoclonic status epilepticus one month after CAR-T therapy.

\section{Results}

A 28-year-old female with a history of chemotherapy-resistant acute lymphoblastic leukemia was admitted with diarrhea and was found to have neutropenic colitis. She had received CAR-T therapy one month prior to presentation, complicated by ICANS with transient encephalopathy and left facial droop treated with tocilizumab, siltuximab, and high-dose steroids. At the time of her readmission, she had intact mental status and motor exam, except for a mild residual left facial weakness. She was treated with broad-spectrum antimicrobials. On day 9, she developed waxing-waning encephalopathy and had a negative non-contrast CT head. Within 12 hours, she was found unresponsive and was intubated for airway protection. She developed generalized myoclonic seizures and EEG revealed status epilepticus refractory to propofol and versed drips, requiring escalation to pentobarbital. LP was negative. CT showed acute diffuse cerebral edema. Subsequent MRI revealed diffuse cortical diffusion restriction consistent with ischemia and edema with central herniation and Duret's hemorrhage. She was treated with high-dose methylprednisolone and anakinra (an IL-1 receptor antagonist) for possible delayed CART neurotoxicity. She developed progressive diffuse cerebral edema with herniation and was pronounced dead on day 16 after neurological examination and radionuclide blood flow study.

\section{Conclusions}

Delayed neurotoxicity following CAR-T therapy may present with rapidly progressive diffuse cerebral edema requiring early high-dose steroids. 


\section{Poster 90}

\section{Real-World Hemostatic Effectiveness of Andexanet Alfa for Management of Intracranial Hemorrhage}

Jennifer C. Wilson, Olivia S. Costa, Craig I. Coleman, Terry Dettling, Richard Dalyai, Susan Samuel, Manjunath Markandaya

Department of Pharmacy, Vidant Medical Center, Greenville, NC, United States

\section{Background \& Purpose}

Andexanet alfa is a factor Xa reversal agent FDA-approved to treat uncontrolled or life-threatening bleeding. We assessed the effectiveness of andexanet in managing intracranial hemorrhage (ICH) in apixaban- or rivaroxaban-treated patients in routine practice.

\section{Methods}

Using electronic health records, we identified consecutive patients admitted to Vidant Medical Center from 1 May 2018-1 February 2021 with computed tomography (CT)-confirmed ICH, presenting <24hours from their last apixaban or rivaroxaban dose and manage

\section{Results}

Seventeen patients with apixaban $(n=12,71 \%)$ or rivaroxaban-associated $(n=5,29 \%)$ ICH were included. Most patients $(n=11,65 \%)$ were receiving anticoagulation for atrial fibrillation. Age (median, interquartile range) was $75(69,80)$ years, admission GCS was $14(13,15)$ and $7(41 \%)$ patients were receiving antiplatelet therapy. Thirteen patients $(76 \%)$ had an intracerebraltintraventricular hemorrhage, one (6\%) a subarachnoid, one (6\%) a subdural, and two patients (12\%) had multicompartmental bleeds (intracerebral+subarachnoid). ICHs were spontaneous in 14 (82\%) patients and $5(29 \%)$ had infratentorial bleeding. Andexanet was administered within $1.3(0.8,3.1)$-hours from initial CT scan and all patients received the low dose. Time-to-repeat CT was $4.5(1.5,8.8)$-hours. Excellent/good hemostatic effectiveness occurred in $14(82 \%, 95 \% \mathrm{Cl}=57-96 \%)$ of patients. One patient $(6 \%, 95 \% \mathrm{Cl}=0.1-29 \%)$ experienced a thromboembolism. Two patients $(12 \%, 95 \% \mathrm{Cl}=15-35 \%)$ died within 30-days.

\section{Conclusions}

Real-world hemostatic effectiveness, thromboembolism and 30-day mortality incidences from this single-center experience of andexanet to manage apixaban- or rivaroxaban-associated ICH appeared consistent with ICH patients in ANNEXA-4. 


\section{Poster 91}

\section{Refractory, Progressive Candida Dubliniensis Ventriculitis, Meningitis and Dilemma of Initial Culture- Negative Cerebrospinal Fluid Culture}

\section{W. David Freeman, Amanda Tomlinson, Sarah Peacock, Sanjeet Grewal, Lisa Brumble, Salvador Alvarez, Jason Siegel, Lauren Ng, Perry Bechtle, Dennis Dickson}

Mayo Clinic Neurosciences Intensive Care Unit, Jacksonville, FL, United States

\section{Background \& Purpose}

Fungal meningitis and ventriculitis is rare in young immunocompetent individuals but more common in those with risk factors such as IV Drug Abuse (IVDA) history, HIV/AIDS, or immunocompromised status. We report two cases of $C$. dubliniensis meningitis with hydrocephalus where one survived and the other did not. A 43 year old male with cirrhosis and controlled diabetes presented with acute progressive encephalopathy. A 35 year old male with a history of IVDA in remission and treated Hepatitis C developed subacute progressive neuropsychiatric disturbance, nausea, vomiting and weight loss.

\section{Methods}

Small case series and review of the literature using National Library of Medicine/Google Scholar search engines using search terms of Candida dubliniensis, hydrocephalus, or meningitis, and/or fluconazole and hydrocephalus.

\section{Results}

Small case series of 2 males with C. dubliniensis hydrocephalus. Using the aforementioned search engine terms and methods, $\mathrm{C}$. dubliniensis and hydrocephalus revealed only 2 published articles, whereas $\mathrm{C}$. dubliniensis and meningitis revealed 7 publications. Overall the literature speaks to a high case fatality rate (CFR) if diagnosis is missed and initial high false negative CSF culture rate. The cirrhosis patient grew C. dubliniensis from his first CSF sample which led to immediate treatment and subsequent shunt placement which allowed a full recovery. The previous IVDA patient workup did not reveal C. dubliniensis until the 11th sample of CSF which led to delay in treatment and ultimately the patient did not survive from intractable ventriculitis.

\section{Conclusions}

C. dubliniensis is a rare CNS cause of meningitis/ventriculitis that may require multimodal infectious disease approach including CSF sampling from multiple sites given differential density of this organism and septations of the ventricular system using metagenomics, Fungitell, and high degree of suspicion. Aggressive neurosurgical interventions are needed and consideration of intrathecal intraventricular antifungal therapy. 


\section{Poster 92}

\section{Retrospective Study of Neurologic Sequelae in Critically III vs Non-Critically III COVID-19 Patients}

Haitham Alabsi, Zeina El-Chemali, George Alba, Leo Ginns, Jonathan Rosand, David Lin

Massachusetts General Hospital, Boston, MA, United States

\section{Background \& Purpose}

To date, over a 150 million people have survived the novel coronavirus 2019 (COVID-19) infection. Sequelae of COVID-19 infection has been heterogenous and have most commonly included fatigue, dyspnea, headache, cognitive impairments, chest pains, mood alterations, smell and taste dysfunction, and myalgia. A letter to Jama noted $87 \%$ of 143 hospitalized COVID patients had ongoing symptoms at 2month follow-up. Neurologic sequelae of COVID-19 are common and disabling (REF). The nature, severity and prevalence of neurologic sequelae of covid-19 is unclear. Furthermore, it is unclear whether critical illness requiring ICU stay is a risk factor for neurologic sequelae. Identifying which patients are at risk for each sequelae can help allocate medical resources and consequently improve patient outcomes.Our primary aim was to characterize neurologic symptoms in a cohort of patients with neurologic sequelae of COVID-19. Our secondary aims were to compare neurologic symptoms in those who were critically ill to those who were not and

\section{Methods}

This is a retrospective characterization of the 66 patients with neurologic sequelae of COVID-19 referred to a Neurorecovery clinic at an Academic Medical Center in Boston, MA. All patient were given a standard interview which included screening for disab

\section{Results}

The median age of patients was 55 for the critical ill and 48 years for the non-crtiically ill . $41 \%$ of the critically ill were females vs $62 \%$ of the non-critically ill. The non-critically ill were noted to have higher disability scores with median MRS score of 2 vs 1 , and permanently left the workforce at a higher rate $58 \%$ vs $11 \%$. Neurologic complaints differed in each group: pain and insomnia were more prominent in the critically ill while headaches, memory/concentration deficits, and dysautonomia were prominent in the non-critically ill.

\section{Conclusions}

Disabling symptoms are noted in both critically ill and non-critically ill patients with neurologic sequelae of Covid-19 infection. Non-critically ill patients were in fact more disabled than critically ill patients at follow-up. 


\section{Poster 93}

\section{Reversible Cerebral Vasoconstriction Syndrome (RCVS): Should we Aim Higher?}

Mahammed Khan Suheb, Amay Parikh, Raad Bassel, Mbeo Gilbert, Okorie Okorie

Advent Health Orlando - Neurocritical Care Department, Orlando, FL, United States

\section{Background \& Purpose}

Reversible cerebral vasoconstriction syndrome (RCVS) is a spectrum of disorders characterized by multifocal reversible narrowing of cerebral arteries. Typical presentations include thunderclap headache which may be associated with seizures, and strokes.

\section{Methods}

A 60-year-old woman presented with recurrent episodes of headache and new onset delirium. She was on Buspirone for anxiety disorder and smoked tetrahydrocannabinol (THC) regularly. Initial CT head and Cerebrospinal fluid analysis were within normal limits

\section{Results}

No consensus exists on management and therapy is based on observational studies and anecdotal evidence. Most experts aim toward using supportive measures, normalizing BP target and using calcium channel blockers like verapamil or nimodipine. Our patients clinical condition worsened by Day 4 despite recommended therapy. We saw improvement in patient's neurological condition after employing BP augmentation in addition to oral nimodipine. With BP augmentation therapy we aim to achieve a higher cerebral perfusion pressure and limit the deleterious effects of cerebral vasoconstriction.

\section{Conclusions}

BP augmentation protocol can be an important tool in the management of RCVS. Further studies are needed to help understand this clinical condition better. 


\title{
Poster 94
}

\section{Reversible Cerebral Vasoconstriction Syndrome Following Cerebral Hyperperfusion Syndrome with Excellent Recovery}

\author{
Eesha Oza, Dattu Vyas, Nirav Vora, Tapan Kavi
}

Medical College of Georgia, Augusta, GA, United States

\section{Background \& Purpose}

Cerebral Hyperperfusion Syndrome (CHS) has been well described after carotid endarterectomy and stenting as a manifestation of impaired cerebral autoregulation. Headache, seizures and focal neurological deficits are seen commonly as a result of CHS. Reactive vasospasm or Reversible Cerebral Vasocontriction Syndrome have not been commonly seen after CHS, but have been reported. We report a case with angiographic evidence of reversible vasoconstriction after CHS and excellent recovery, which has not been reported before.

\section{Methods}

This patient was identified through presentation to the Neuro-ICU

\section{Results}

A 54-year-old female with history of Hypertension and left Retinal Artery occlusion underwent left Carotid endarterectomy for severe symptomatic stenosis. One week later she suffered seizures and acute respiratory failure. MRI brain revealed a small acute left insular ischemic stroke surrounded by hyperintensity suggestive of hyperperfusion syndrome. A CT angiogram obtained for further evaluation of the stroke revealed vasospasm in left Anterior Cerebral Artery (ACA) and Middle Cerebral Artery (MCA). This vasospasm was confirmed on conventional cerebral angiogram with moderate spasm in left ACA and MCA. Given the stability of neurologic exam, the patient was treated with permissive hypertension during this period. A repeat CT angiogram two days later showed resolution of spasm. Patient did not have any further seizures and was discharged home with minimal weakness on right side.

\section{Conclusions}

Managing Blood Pressure (BP) in cases with concurrent CHS and RCVS can be difficult because of the need to maintain adequate perfusion through constricted intracranial circulation while avoiding worsening of cerebral edema and hemorrhagic conversion in the hyperperfused tissue. However, the self-limiting course of vasospasm may suggest the need for higher BP goals to be transient. Case-series or other larger scale studies may help support this observation and corresponding management. 


\title{
Poster 95
}

\section{Reversible Cerebral Vasoconstriction Syndrome in Patients with Coronavirus Disease: A Multicenter Case Series}

\begin{abstract}
Anna M Cervantes-Arslanian, Shilpa Samudrala, Mohamad Abdalkader, Pria Anand, Ali Daneshmand, Hormuzdiyar Dasenbrock, Thanh Nguyen, Charlene Ong, Courtney Takahashi, Julie Shulman, Marc Alain

Babi, Sanjeev Sivakumar, Neel Shah
\end{abstract}

Boston University School of Medicine, Boston Medical Center, Boston, MA, United States

\section{Background \& Purpose}

Background/Purpose: RCVS (reversible cerebral vasoconstrictive syndrome) is a condition associated with vasoactive agents that alter endothelial function. There is growing evidence that endothelial inflammation is one of the pathways that contributes to acute ischemic stroke in patients with coronavirus disease 2019 (COVID-19). In our study, we describe the clinical features, risk factors, and outcomes of RCVS in a multicenter case series of patients with COVID-19.

\section{Methods}

Multicenter case series. We collected clinical characteristics, imaging, and outcomes of patients with RCVS and COVID-19 identified at each participating site.

\section{Results}

Ten patients were identified, 7 women, ages $21-62$ years. Risk factors included use of vasoconstrictive agents in 7 and history of migraine in 2. Presenting symptoms included thunderclap headache in 5 patients and recurrent headaches in 4. Eight were hypertensive on arrival to the hospital. Symptoms of COVID-19 included fever in 2, respiratory symptoms in 8 , and gastrointestinal symptoms in 1 . One patient had no COVID-19 symptoms. MRI showed subarachnoid hemorrhage in 2 cases, intraparenchymal hemorrhage in 1, acute ischemic stroke in 3, FLAIR hyperintensities in 3, and no abnormalities in 1 case. Neurovascular imaging showed focal segment irregularity and narrowing concerning for vasospasm of the left MCA in 4 cases and diffuse, multifocal narrowing of the intracranial vasculature in 6 cases. Outcomes varied, with 2 deaths, 2 remaining in the ICU, and 6 surviving to discharge with modified Rankin scale (mRS) scores of $0(n=2), 1(n=2), 2(n=1)$, and $3(n=1)$.

\section{Conclusions}

Our series suggests that patients with COVID-19 may be at risk for RCVS, particularly in the setting of additional risk factors such as exposure to vasoactive agents. There was variability in the symptoms and severity of COVID-19, clinical characteristics, abnormalities on imaging, and mRS scores. However, a larger study is needed to validate a caUnited Statesl relationship between RCVS and COVID-19. 


\title{
Poster 96
}

\section{Septic versus Aseptic Meningitis in a Patient with Untreated Systemic Lupus Erythematosus}

\author{
George Manuel Lu, Shouri Lahiri, Michael Gezalian, Maranatha Ayodele, Shahed Toossi
}

Cedars-Sinai Medical Center Neurocritical Care, Los Angeles, CA, Unites States

\begin{abstract}
Background \& Purpose
Systemic Lupus Erythematosus (SLE) can disrupt the blood-brain barrier, a condition termed neuropsychiatric SLE (NPSLE). Given its diverse phenotypic presentation, diagnosing NPSLE in patients presenting with meningitis poses diagnostic and therapeutic challenges.
\end{abstract}

\section{Methods}

Retrospective chart review.

\section{Results}

A 37 year old female, with history of transverse myelitis, cerebral venous sinus thrombosis, left temporal lobe hemorrhage, chronic paraplegia, presumed SLE (not on immunosuppression), presents with fevers, progressive encephalopathy, ophthalmoplegia and bilateral arm weakness. On exam, she presented febrile, markedly tachycardic, lethargic, anarthric, with bifacial weakness, absent corneal and gag reflexes. She was intubated for airway protection. Head computed tomography (CT) demonstrated pontine-midbrain hypodensities with left temporal lobe gliosis. CT angiogram was normal. Broad spectrum antibiotics were initiated. Within an hour of presentation, she became comatose with decerebrate posturing. A lumbar puncture (LP) yielded an opening pressure of $25 \mathrm{cmH} 20$, white blood cells-212 (91\% polynuclear cells), red blood cells-0, protein-85, glucose-32 (serum glucose-132mg/dl). Magnetic resonance imaging (MRI) brain demonstrated non-enhancing $\mathrm{T} 2$ hyperintensity in the brainstem structures with associated diffusion restriction and microhemorrhages. Given concern for septic meningitis with atypical MRI findings, repeat LP was performed, which demonstrated improving leukocytosis and negative cultures. Serum immunologic studies revealed elevated ANA-(1:640) and dsDNA-(1:640), and low rheumatoid factor- $(<15 \mathrm{lU} / \mathrm{ml}), \mathrm{C} 3-(38 \mathrm{mg} / \mathrm{dl})$ and C4-(12mg/dl). Therapeutic approach included methylprednisolone, plasma exchange, rituximab and prednisone taper. Days later, patient was alert, following commands, limbs antigravity with dysmetria, improved ophthalmoparesis and facial weakness.

\section{Conclusions}

This case demonstrates the importance of a good history and consideration of repeating studies where there is diagnostic uncertainty. Although our patient's presentation raised concern for central nervous system (CNS) infection, her history, serologic studies, and neuroimagings supported a diagnosis of NPSLE. Additionally, finding serologic hypocomplementemia was strongly suggestive of NPSLE. Distinction between NPSLE and CNS infection is crucial to minimize risk of morbidity and mortality. 


\section{Poster 97}

\section{Spinal Reflexes Precluding Brain Death Diagnosis: A Case Report}

Michael Hadler, Anand Karthik Sarma

Wake Forest Baptist Medical Center, Department of Neurology, Winston Salem, NC, United States

\section{Background \& Purpose}

Spinal reflexes and automatisms in brain death patients is well-established and does not preclude the diagnosis of brain death. These movements can delay brain death diagnosis, particularly when suggestive of intact brainstem function. We describe an interesting case of a now 11-month-old infant with spinal reflexes in response to cranial nerve stimulation, confounding the diagnosis of brain death.

\section{Methods}

Data was collected by clinical observation.

\section{Results}

A two-month-old female was admitted to the Pediatric Intensive Care Unit after witnessed cardiac arrest. Initial neurologic exam demonstrated absent brainstem reflexes, hypotonia, no spontaneous movement and no response to noxious stimulation. After initial myoclonic status epilepticus resolved, EEG was isoelectric. On hospital day 5, formal brain death testing demonstrated absent brainstem reflexes but truncal movements during apnea test were interpreted as either spinal reflex or respiratory effort. Nuclear medicine brain perfusion test showed that blood flow to the brain remained intact. Repeat EEG on hospital days 141 and 235 remained isoelectric. On hospital day 246, neurological exam demonstrated triple flexion and contraction of abdominal wall in response to peripheral noxious stimulation. Brainstem reflexes remained absent, but the patient demonstrated triple flexion of the right lower extremity in response to tactile stimulation of the cornea and endotracheal suction. This response was deemed inconsistent with a diagnosis of brain death, therefor planned repeat formal brain death testing and ancillary testing was not pursued.

\section{Conclusions}

This unique case raises interesting questions. While reflexive movements in brain death patients typically abate within 72 hours, they were present on day 246 in our patient. Also, no infant less than one-year-old has ever been reported to demonstrate reflexive movements in brain death. Should these movements supersede our other findings and be taken to indicate intact brain function, or should we consider these movements a limited finding in the context of significant evidence for brain death? 


\title{
Poster 98
}

\section{Spontaneous Conus Medullary Infarction in the Absence of Cardiovascular Risk Factors}

\author{
Bavica Gummadi, Jaffer Ahmed, Swarna Rajagopalan
}

Medical student, Rowan School of Medicine, Stratford, NJ, United States

\section{Background \& Purpose}

Spinal cord infarction $(\mathrm{SCl})$ is a rare but devastating disease that occurs in association with trauma or predisposing cardiovascular risk factors. Since clinical presentation is variable, it remains a diagnostic challenge in patients without risk factors, often mistaken for other diseases. Delayed diagnosis of $\mathrm{SCl}$ can result in missed treatment opportunities and contribute to the high case fatality rate. This case report examines the case of a 42-year-old man with no significant past medical history who presented with clinical symptoms of spinal cord compression secondary to acute $\mathrm{SCl}$ in the setting of heavy lifting.

\section{Methods}

A 42-year-old man with no medical history presented with sudden lower back pain when lifting a heavy box followed by progression of bilateral leg paralysis over several hours, saddle anesthesia, and urinary retention. On examination, he was paraplegic wit

\section{Results}

Initial urgent MRI with T2-weighted images of the thoracolumbar spine demonstrated no clear pathology. Repeat MRI of thoracolumbar spine a day later revealed hyperintensities on T2-weighted imaging in the conus medullaris suggestive of a SCl. At this time, the diffusion weighted imaging (DWI) also demonstrated restriction consistent with acute infarct.

\section{Conclusions}

In considering $\mathrm{SCl}$ in the setting of spinal shock, DWI imaging can be promptly pursued to make a timely and definitive diagnosis. Although T2-weighted images are obtained initially, the sensitivity of MRI is limited in the first several hours of infarct, making DWI the preferred imaging technique. Additionally, with timely diagnosis of $\mathrm{SCl}$, thrombolytic therapy can be considered as a treatment modality as published case reports demonstrate good functional recovery. This case report highlights the importance of including $\mathrm{SCl}$ in the differential of a patient with acute paraparesis even in the absence of co-existing risk factors. 


\section{Poster 99}

\section{Spontaneous ICH in New Onset IgA Nephropathy: A Case Report}

Danielle A. Bazer, Matthew Orwitz, E. Wirkowski

Renaissance School of Medicine at SUNY Stony Brook, Department of Neurology, Stony Brook, NY, United States

\section{Background \& Purpose}

IgA nephropathy, formerly known as Berger's Disease, is the result of nephritic immune complexes that are both circulating and glomerular. The most common presentation is asymptomatic hematuria and progressive kidney disease. The etiology is believed to be both genetic and environmental. The relationship between nephritic syndrome and intracranial hemorrhage is not well defined. In comparison, nephrotic syndromes are known stroke risk factors due to creating a hypercoagulable state. We present a case with cerebellar hemorrhage as the presenting symptom for IgA nephropathy.

\section{Methods}

$\mathrm{N} / \mathrm{A}$

\section{Results}

A 25 year-old-male with no previous medical history initially presented due to first time tonic-clonic seizure in the setting of flu-like illness. The patient required intubation for airway protection in the field. On head CT, the patient had a right cerebellar hemorrhage with surrounding edema, as well as scattered supratentorial ischemic strokes. The patient required emergent evacuation of the cerebellar hemorrhage due to the mass effect. Concomitantly upon presentation, the patient was in acute renal failure as well as hypertensive emergency, requiring continuous intravenous antihypertensive therapy. To elucidate the etiology of the acute renal failure, the patient had a renal biopsy, which revealed endocapillary proliferation and crescents with IgA, IgM, and IgG immune deposits in the mesangium and subendothelial area, consistent with IgA nephropathy. Urine studies acutely and in follow-up never revealed nephrotic range proteinuria.

\section{Conclusions}

Presentation of acute neurological pathology in IgA nephropathy has not prior been described in the literature. This patient lacked nephrotic range proteinuria which is commonly associated with ischemic strokes, and rarely hemorrhagic stroke. It is likely that his IgA nephropathy contributed to the development of hypertensive emergency and subsequent intracranial hemorrhage. 


\title{
Poster 100
}

\section{Spontaneous Spinal Subarachnoid Hemorrhage with Simultaneous Intracranial Subarachnoid Hemorrhage: A Case Report}

\author{
Shawn Moore, Mohammad Hafeez, Khwaja Siddiqui, Chethan Venkatasubba Rao \\ Baylor College of Medicine, Houston, TX, United States
}

\section{Background \& Purpose}

Spontaneous spinal subarachnoid hemorrhage $(\mathrm{SAH})$ is a rare condition that can lead to significant neurologic deficits. We report a case of a simultaneous intracranial and spinal SAH who developed cord compression resulting in paraplegia and requiring spinal decompression.

\section{Methods}

Existing literature was searched using PubMed, Embase and Google Scholar using the terms spinal subarachnoid hemorrhage and intracranial subarachnoid hemorrhage.

\section{Results}

A sixty-three-year-old female with history of end stage renal disease (ESRD) caused by polycystic kidney disease (PKD) who presented with abdominal pain followed by headaches, neck pain, numbness from umbilicus to toes, nausea and vomiting. Initial CT head revealed a diffuse SAH. During evaluation in emergency room, she suddenly became paraplegic and more somnolent. Physical examination revealed a T10 spinal sensory level, absent patellar and Achilles reflexes, and paraplegia. MRI of spine revealed SAH tracking along cervical and thoracic spinal cord. There was a large hematoma resulting in cord compression at T10 spinal level requiring urgent surgical decompression. Diagnostic cerebral and spinal angiograms were negative for any aneurysm or vascular malformation. Hematologic evaluation with mixing study was concerning for potential vitamin $\mathrm{K}$ dependent factor insufficiency. In addition, uremic platelet dysfunction secondary to ESRD was thought to be a contributing factor to her coagulopathic state.

\section{Conclusions}

There is limited literature available on simultaneous intracranial and spinal SAH. Recognizing early signs of spinal cord injury in such patients can lead to timely interventions. There are few case reports identifying anterior spinal artery aneurysms, including one case report in a patient with PKD, as etiology for spontaneous spinal SAH. In our case, angiograms were negative for any aneurysms, but possibility of a small aneurysm cannot be entirely excluded. In additional, her underlying coagulopathic status and platelet dysfunction likely played a role in development of extensive SAH. 
Poster 101

Suddenly Breathless: Ondine's Curse Following Listeria Rhomboencephalitis

Michael Sansait, Dilpreet Singh, Clara Weinstein, Benjamin Johnson, William McGee

Baystate Medical Center, Springfield, MA, United States

\section{Background \& Purpose}

Listeria rhombencephalitis (LRE) is a rare central nervous system infection of the brain stem. It is characterized by febrile illness, abrupt onset of asymmetric cranial nerve palsies and encephalopathy. Acute respiratory failure manifesting as acquired Ondine's curse has rarely been reported.

\section{Methods}

We report a case of Listeria rhombencephalitis in a 67-year-old male presenting with cranial nerve palsies, encephalopathy, recurrent sudden apnea and persistent hypercapnia due to autonomic respiratory failure. Lumbar puncture, blood cultures, and magnet

\section{Results}

Blood cultures, lumbar puncture and MRI of the brainstem were ordered. Ampicillin and gentamicin was started as empiric antimicrobial therapy. Blood cultures and cerebrospinal fluid cultures grew Listeria monocytogenes. Brain MRI without contrast revealed a $4 \mathrm{~mm}$ focal abnormality in the left medulla with a small amount of surrounding hemorrhage and edema and with gadolinium MRI showed the same left medullary lesion with ring enhancement, multiple areas of enhancement throughout the brainstem and leptomeninges. Localization of the lesion led to recognition of acquired central alveolar hypoventilation syndrome as the cause of the patient's acute respiratory failure.

\section{Conclusions}

Acute respiratory failure and neurological sequelae are a result of the brainstem lesion caused by Listeria monocytogenes infection. This life-threatening complication has variable prognosis with unpredictable recovery. LRE should prompt initiation of antimicrobial treatment, reducing the risk of brainstem involvement and its life-threatening sequelae. MRI identifies the characteristic brainstem lesions. Supportive care using mechanical ventilation in the setting of central hypoventilation syndrome can be lifesaving. 


\section{Poster 102}

\section{Takotsubo Cardiomyopathy after Traumatic Brain Injury}

Fajun Wang, Joseph Darby

Department of Critical Care Medicine, University of Pittsburgh Medical Center, Pittsburgh, PA, United States

\section{Background \& Purpose}

Takotsubo cardiomyopathy (TCM) or "stress cardiomyopathy" is an uncommon condition characterized by transient cardiac dysfunction with left ventricular apical ballooning in an appropriate clinical context. TCM has been observed in a variety of acute neurological conditions most prominently in patients with aneurysmal subarachnoid hemorrhage and status epilepticus. TCM has only been reported infrequently in association with traumatic brain injury (TBI). Herein we present a patient who developed TCM 3 days after hospital admission with severe TBI.

\section{Methods}

A retrospective chart review was performed on the patient's medical records. We present a case of Takotsubo cardiomyopathy after traumatic brain injury.

\section{Results}

A 30-year-old male presented to the hospital with an acute subdural hematoma, anisocoria, declining consciousness and CT evidence of uncal herniation after being found down in a hotel room. The patient was taken emergently to the operating room for decompressive hemicraniectomy and hematoma evacuation. On the post-trauma day (PTD) 3, the patient developed acute dyspnea with increased oxygen requirements that improved with diuresis. On PTD 4, nursing staff noted T waive inversions (TWI) on the bedside monitor prompting an electrocardiogram (ECG) that showed a prolonged QTC interval and worsening TWI in leads I, II, aVL, and V2-6. Troponin I level was mildly elevated at $0.63 \mathrm{ng} / \mathrm{mL}$. Transthoracic echocardiography (TTE) was subsequently performed and showed a low ejection fraction (EF 26\%) with apical hypokinesis and basal hyperkinesis, consistent with TCM. A diagnosis of TCM was confirmed by Cardiology consultation and he was started on a beta-blocker and an ACE inhibitor. Follow-up TTE on PTD 20 showed a normal left ventricular EF.

\section{Conclusions}

While rarely reported in patients with TBI, TCM developed in an otherwise healthy young male following severe TBI necessitating decompressive hemicraniectomy. TTE should be considered in patients with TBI who have cardio-pulmonary symptoms or unexplained EKG abnormalities. 
Poster 103

Takotsubo Cardiomyopathy in Aneurysmal Subarachnoid Hemorrhage

Fatima Ali, Neveen Malik

Rowan University School of Osteopathic Medicine and Jefferson Health/ Pulmonary and Critical Care, Stratford, NJ, United States

\section{Background \& Purpose}

Takotsubo cardiomyopathy (TCM) is a reversible cardiomyopathy characterized by left ventricular apical ballooning. TCM can be found in $1.2 \%$ to $26 \%$ of patients with subarachnoid hemorrhage (SAH). The neuro-cardiac injury of SAH is an important complication to recognize, especially in the setting of cerebral vasospasm or delayed cerebral ischemia. The following case discusses a patient with a large SAH and subsequent development of TCM.

\section{Methods}

A 71-year-old female with history of hypertension presented to the emergency department after being found unresponsive with a GCS of 3 in her car and subsequently intubated. On neurological exam, patient was found to have an intact respiratory drive, dece

\section{Results}

Patient underwent a diagnostic cerebral angiogram and coiling of the L PICA aneurysm with placement of an external ventricular drain. Post procedure, patient was noted to be hypotensive requiring high dose vasopressor therapy to maintain MAP $>65$. An EKG was done showing T wave inversion in leads V2 to V4. Troponins were elevated to $1814 \mathrm{ng} / \mathrm{l}$ (normal <18). Bedside cardiac ultrasound revealed severe global akinesis consistent with takotsubo cardiomyopathy.

\section{Conclusions}

SAH-induced TCM is a reversible cardiomyopathy not due to a primary defect in myocardial perfusion. A massive release of catecholamines into the systemic circulation after aneurysmal rupture is considered responsible for SAH-induced TCM. Increased risk factors for developing SAH-induced TCM include elevated CK-MB levels of $\geq 2 \%$, poor neurologic status and female gender. There is increased mortality in patients with TCM associated SAH. Treatment can be challenging since treatment for TCM can have detrimental effects on cerebral perfusion in the setting on SAH. 


\title{
Poster 104
}

\section{The First CNS Gamma-Delta T-Cell Lymphoma/leukemia; A Case Report}

\author{
Sepideh Chagharvand, Marin Darsie
}

University of Wisconsin Hospitals and Clinics, Neurology Department, Madison, WI, United States

\section{Background \& Purpose}

Natural killer/T-Cell lymphoma (NKTCL) is overall rare, and there are only 5 reports of CNS involvement. Gamma-delta T cell lymphoma/leukemia is an extremely rare subtype of NKTCL $(<1 \%)$, and to the best of our knowledge, there are no case reports associated with CNS involvement.

\section{Methods}

N/A

\section{Results}

A 50-year-old man was admitted with confusion and new-onset seizures. He underwent extensive workup for persistent encephalopathy which demonstrated significantly elevated inflammatory markers, limbic system, and multiple CN FLAIR changes on brain MRI, as well as hepatomegaly on body imaging. LP was performed which illustrated 657 cells (33\% neutrophils, 32\% lymphocytes, 35\% macrophages), protein 289 , glucose 30 , with negative viral panel and cultures. His clinical course declined despite empiric bacterial meningoencephalitis management, pulse steroids, IVIg, and Tocilizumab. The patient developed multisystem organ failure including transaminitis, acute kidney injury, and pancytopenia. The bone marrow, liver, and brain biopsies initially were non-diagnostic, however abnormal cytogenetic results of bone marrow (H21-393) prompted special immunostaining and flow cytometry, which demonstrated positive CD2, CD3, CD56, and circulating gamma-delta lymphoma/leukemia. These findings are consistent with a very aggressive subtype of NKTCL, known as gamma-delta T cell lymphoma/leukemia. Given the poor prognosis $<2$ months, he was transferred to palliative care and subsequently died on HD 54.

\section{Conclusions}

Gamma-delta T cell lymphoma/leukemia is a very rare subtype of NKTCL, that has been associated with skin, lymph node, and liver involvement. Per 2020 WHO NKTCL classification, there has never been a reported case of CNS involvement with this subtype. The precipitous decline in the health status of this patient fits with the extremely poor prognosis reported for the other subtypes of NKTCL. Routine use of cytogenetics may ensure the diagnosis of extremely rare lymphomas/leukemias in the setting of the limits of traditional pathology testing. CNS: Central nervous system, NKTCL: Natural killer/T cell lymphoma, CN: Cranial nerves, LP: Lumbar puncture, IVlg: Intravenous immunoglobulin, HD: Hospital day, WHO: World health organization. 


\title{
Poster 105
}

\section{The Use Of Siltuximab in Chimeric Antigen Receptor T-cell Neurotoxicity and Neuro-inflammation in Critically III Patients}

\author{
Kaylee Marino, Brian Schuler, Alison Yu, Leslie Kean, Yasmin Aghajan, \\ Brigham and Women's Hospital, Boston, MA, United States
}

\section{Background \& Purpose}

Siltuximab is a human-murine chimeric monoclonal antibody that binds to and antagonizes interleukin- 6 (IL-6). While only currently FDA approved for the treatment of multicentric Castleman's disease, siltuximab has been trialed in neurologic conditions with elevated concentrations of IL- 6 including chimeric antigen receptor T-cell (CAR-T) neurotoxicity, central nervous system vasculitis, and encephalitis. The purpose of this study was to analyze clinical outcomes as well as cerebrospinal fluid (CSF) inflammatory cytokine levels pre- and post-treatment with siltuximab.

\section{Methods}

This was a single-center, retrospective analysis performed at a tertiary academic medical center and approved by the hospital's Institutional Review Board. This study identified critically ill patients admitted to the neurosciences intensive care unit who

\section{Results}

Eight patients received siltuximab for indications of CAR-T neurotoxicity $(n=6)$, granulomatous CNS vasculitis $(n=1)$, and acute necrotizing encephalitis $(n=1)$. Three patients had serial CSF sampling before and after treatment. CSF cytokines including IL-6, interleukin-10 (IL-10), tumor necrosis factor alpha (TNF-a), interferon alpha (IFN-a), interferon gamma (IFN-g), and granulocyte-macrophage colonystimulating factor (GM-CSF) decreased in the patients treated for CAR-T neurotoxicity and corresponded to rapid clinical improvement. Cytokine levels did not show a downward trend in the patients treated for vasculitis or encephalitis, corresponding to poor clinical outcomes. In the 6 patients who received siltuximab for CAR-T neurotoxicity, three had neurologic recovery while the other three died due to their underlying medical condition.

\section{Conclusions}

Siltuximab may be considered in the treatment of patients with CAR-T neurotoxicity. Limited CSF data suggests IL-6, IL-10, TNF-a, IFN-a, IFN-g, and GM-CSF may play a role in the CAR-T neurotoxicity disease process. More data is needed to evaluate siltuximab as a treatment option in other neuro-inflammatory conditions including vasculitis and encephalitis. 


\section{Poster 106}

Thymoma Associated with Autoimmune Encephalitis and Subsequent Myasthenia Gravis.

Syed F. Ali, Tyler D'Agostino, Daniela Galluzzo, Angeles Clara, Patrick Gregory, Rahman Mozibur, Serena Wong, Eric Feldstein, Theresa Henson, Jin Li

Neurology, New York Medical College, NY, United States

\section{Background \& Purpose}

Thymomas are known to be associated with paraneoplastic syndromes, such as myasthenia gravis and autoimmune encephalitis. However, two distinct paraneoplastic syndromes associated with thymoma in one patient are rarely reported. We report a case of multifocal cortical encephalitis and myasthenia crisis associated with thymoma, which required a prolonged neurocritical intensive care.

\section{Methods}

Case Report.

\section{Results}

A 42-year-old female had recent history of COVID-19 infection and glutamic acid decarboxylase (GAD)65 seropositive multifocal encephalitis 3 month prior to the current presentation of one week of shortness of breath. She was treated with IVIG and steroid for encephalitis; and on lacosamide and levetiracetam for generalized tonic clonic seizures. On admission, physical exam revealed left ptosis, intermittent diplopia, and neck weakness. Chest computed tomography revealed an elevation of left hemi-diaphragm. Electrodiagnostic test showed significant decrement on repetitive stimulation. MRI of the chest revealed thymoma. MRI Brain showed new encephalitis lesions. She was positive for antibodies against binding, blocking and modulating acetylcholine receptors, anti-striated muscle and GAD65. She developed hypercapnic respiratory failure requiring emergent intubation, and subsequently underwent robotic assisted radical thymectomy. The cytology of the mediastinal mass was confirmatory for WHO Grade B1 thymoma with abundant CD3+ T-cells thymocytes. She showed partial improvement in response to immunotherapy with IVIG, steroids and plasmapheresis. She was started on azathioprine for maintenance and discharged to rehabilitation unit.

\section{Conclusions}

Thymoma associated paraneoplastic syndromes of multifocal cortical encephalitis and subsequent myasthenia gravis may present in one person. Thymectomy and aggressive immunotherapy are required to achieve clinical improvement. 


\title{
Poster 107
}

\section{Unique Presentation of Advanced Neurosyphilis with Unusual Neuroradiographic Findings}

\author{
Spencer J. Craven, Zachary Threlkeld, Kyle A. Lyman, Angie Murkins, Karen Hirsch \\ Stanford Health Care, Department of Neurology, Palo Alto, CA, United States
}

\section{Background \& Purpose}

Cases of late syphilis in the United States are rising despite a decline in the overall incidence of syphilis. Neurosyphilis, characterized by central nervous system involvement by Treponema pallidum, may manifest between 1 and 30 years after initial infection. Diagnosis requires a high index of suspicion. We describe a case of late neurosyphilis presenting with status epilepticus, early neurologic decline, and unusual neuroimaging findings.

\section{Methods}

Case presentation and literature review.

\section{Results}

A 44-year-old male was admitted to the Neuro-ICU with status epilepticus after two years of progressive cognitive decline and psychiatric symptoms. After acute management of status epilepticus, examination demonstrated receptive and productive aphasia, coprolalia, right facial droop, hypertonia, gait ataxia, and a profound startle response. MRI brain demonstrated prominent bilateral anterior temporal lobe subcortical FLAIR hyperintensities and an acute left thalamic infarct on DWI. Initial differential diagnosis included leukoencephalopathies, including cerebral autosomal dominant arteriopathy with subcortical infarcts and leukocencephalopathy (CADASIL). Electroencephalography showed lateralized periodic discharges with fast activity that improved with anti-seizure medication. CSF showed a lymphocytic pleocytosis and elevated protein. CSF treponema pallidum antibody IgG was positive with RPR titer of 1:256. Testing was negative for viral, fungal and autoimmune encephalitides, Lyme, Creutzfeldt-Jacob Disease, and HIV. He was diagnosed with neurosyphilis with neurovascular involvement, and appropriately treated with IV penicillin G. The patient's symptoms showed early improvement before hospital discharge.

\section{Conclusions}

Given its rarity and protean manifestations, diagnosis of neurosyphilis requires a high index of suspicion and early treatment is important. Neuroradiographic findings of neurosyphilis may overlap with other degenerative diseases and the clinical findings are non-specific. Thus neurosyphilis should be considered in patients presenting with cognitive decline at a young age, otherwise unexplained psychiatric changes, and temporal white matter changes. Late neurosyphilis carries a poorer prognosis, and treatment frequently leads to incomplete recovery. 


\title{
Poster 108
}

\section{Vascular Disaster: Aortic Dissection and Thrombotic Stroke}

\author{
Adriana Mendez, Jennifer Axelband
}

St. Luke's University Health Network Neurology Residency, Bethlehem, PA, United States

\section{Background \& Purpose}

Management of aortic dissection with carotid artery thrombus presents as a challenge as optimal management is unknown. This case outlines critical factors in management and multidisciplinary collaboration when providing patient care for diagnoses with simultaneously opposing hemodynamic goals.

\section{Methods}

Chart review of a recently multidisciplinary case

\section{Results}

A 65-year-old female with history of hypothyroidism presented to the emergency department (ED) with one hour of left-sided weakness and dysarthria proceeded by vomiting. She had and initial NIH stroke scale of 23 along with bradycardia and hypotension. CT head was unrevealing. CTA head and neck showed a distal right M1 and proximal M2 occlusion with intraluminal thrombus extending from the right bifurcation into the right internal carotid artery (ICA) along with diffuse thickening of the entire length of the aortic wall extending from the valve plane through the diaphragmatic hiatus. Active area of extravasation of contrast from the aortic lumen into the adjacent wall and mediastinum was identified. CTA dissection protocol confirmed a Type A aortic intramural hematoma extending into the arch initially without an identifiable intimal flap. Neurocritical Care, Neurology, Cardiothoracic surgery (CTS) and endovascular neurosurgery (ENS) agreed to proceed to ENS thrombectomy and defer CTS interventions until neurologic stability. Angiography identified severe tortuosity with multifocal kinking, stenosis and areas of dilatation within the right ICA compatible with fibromuscular dysplasia. A large partially occlusive thrombus spanning the right external carotid artery and extending into the right ICA contiguously to the right middle cerebral artery bifurcation. Mechanical $\mathrm{TICl}$ 3 thrombectomy with no residual intraluminal thrombus was achieved.

Given patient's aortic dissection and active bleeding as well as acute stroke findings, managing an appropriate hemodynamic balance to maintain perfusion and prevent worsening ischemia as well as preventing worsening of dissection/mediastinal bleed created significant challenges in this patient's management. Hemodynamic goals were modified for both diagnoses with teams compromising on MAP $>85$ and SBP 140 with HR $<80$. Neurologically our patient stabilized, and a repeat CTA of the aorta was performed showing an area likely representing an intimal tear. She subsequently underwent thoracic endovascular aortic repair (TEVAR) without any complications. She recovered and stabilized permitting discharge to rehab.

\section{Conclusions}

Patients presenting with complex vascular neurology presentations require a collaborative multidisciplinary approach to patient care for best patient outcomes and survival. 
Poster 109

\section{Acute Bacterial Meningitis Risk Factors in Patients with Craniocerebral Gunshot Injuries}

Himani Sood, Ali Daneshmand, Anna M. Cervantes-Arslanian, Joanna Yang, Kevin Yeh, Cynthia A. Rupp, Hormuz Dasenbrock, Ala Nozari, Tejal S. Brahmbhatt, Kushak Suchdev

Boston University School of Medicine, Boston, MA, United States

\section{Background \& Purpose}

Craniocerebral Gunshot Injuries (CGI) can predispose patients to acute bacterial meningitis (ABM). Antibiotics are often given prophylactically, although evidence supporting their utility is limited.

\section{Methods}

We reviewed 4,574 patients presenting with traumatic brain injury to our institution between 2013 and 2019 and determined the incidence of meningitis, antibiotic use and risk factors for ABM. Patients were screened for ABM within six weeks following admis

\section{Results}

80 patients $(1.75 \%)$ had CGI, of whom $55.56 \%(n=50)$ survived the first 24 hours. Twenty patients were given CNS dose prophylactic antibiotics with a mean antibiotic duration of 5.6 days. None of the patients on antibiotics had CSF leak. Of the thirty patients who were not given prophylactic antibiotics, seven patients were diagnosed with ABM, based on neuroimaging for one patient and CSF findings for the rest. Basilar skull fractures with CSF leak were present in three out of seven patients with ABM. Six out of seven patients survived the hospitalization and were discharged to rehab. The incidence of $A B M$ was $23.33 \%$ in CGI patients who did not receive antibiotics. Patients with a CSF leak had a significantly higher risk of developing ABM compared to patients without a CSF leak [Odds Ratio (OR) 6.50, 95\% Confidence Interval (CI) 1.35-201.29]. ABM was also higher in patients with basilar skull fracture [OR: $5,95 \% \mathrm{Cl} 0.73$ 34.35], although the difference was not significant.

\section{Conclusions}

Our data suggest that CSF leak is associated with and a potential risk factor for ABM in CGI patients. Patients on prophylactic antibiotics did not develop ABM; however, none of those patients had a CSF leak, which may have put them at lower risk for developing ABM. Future prospective studies, especially in patients with CSF leaks, are needed to confirm our findings. 


\title{
Poster 110
}

\section{Burden of CNS Infections in Civilian Penetrating Traumatic Brain Injury: A Nationwide Inpatient Sample Analysis}

\author{
Shyam Sudarshan Rao, Janine Molino, Ali Basil Ali, Aoife Reilly, Ayaz Khawaja, Ali Mahta, Bradford \\ Thompson, Tracy Madsen, Linda Wendell, Michael Reznik, William B. Gormley, Saef Izzy \\ Alpert Medical School of Brown University, Neurology, Providence, RI, United States
}

\section{Background \& Purpose}

Penetrating traumatic brain injury (pTBI), commonly from gunshot wounds has been associated with high mortality rates. CNS infections are a potentially fatal yet preventable complication of pTBI, however the data available is limited to single center case series and predominantly in military war scenarios.

\section{Methods}

Hospitalized patients with gunshot wounds to the head between 2011 and 2014 were studied using the National Inpatient Sample. Poor outcomes include death and discharge to a skilled nursing facility. Logistic regression models that account for the complex

\section{Results}

In total 2,118 unweighted pTBI hospitalizations, representing a population of 10,492 pTBI hospitalizations, were extracted (mean age 48.8 years; $15.2 \%$ female; $49.7 \%$ Caucasian). CNS infections occurred in $1 \%$ of pTBI hospitalizations $(0.29 \%$ meningitis, $0.67 \%$ ventriculitis, $0.83 \%$ intracranial abscess, and $0.05 \%$ subdural empyema). The likelihood of CNS infection was higher among patients with a larger number of chronic health conditions, (OR=1.29, 95\% Cl=1.14-1.45, $p<0.0001)$, a CSF leak (OR=11.30, 95\% $\mathrm{Cl}=3.17-40.28, \mathrm{p}=0.0002)$, and diabetes $(\mathrm{OR}=4.54,95 \% \mathrm{Cl}=1.31-15.73, \mathrm{p}=0.02)$. Patient age and gender, substance abuse, length of stay, and skull fracture were not associated with the likelihood of CNS infection. Poor outcomes occurred in $77 \%$ of pTBI hospitalizations. Although it did not attain statistical significance, CNS infections appeared to increase the likelihood of poor outcomes (OR=5.90, 95\% $\mathrm{Cl}=0.79-44.07, \mathrm{p}=0.08)$.

\section{Conclusions}

CNS infection is a serious complication of civilian pTBI, associated with chronic health conditions, diabetes and CSF leak. CNS infections may contribute to the burden of poor outcomes in pTBI. 
Poster 111

COVID-19

Janneke Horn, Chandeny G. Bennewitz, Lisa Vermunt, Charlotte E. Teunissen

Amsterdam University Medical Center, Intensive Care, Amsterdam, Netherlands

\section{Background \& Purpose}

COVID-19 primarily affects the respiratory system, however neurological complications have been reported too. It is unknown which diagnostic tests are most useful for brain injury identification in COVID-19 patients. We investigated the clinical practice of neurological diagnostics, including neurofilament light (NfL), in intensive care unit (ICU) patients from the first COVID-19 wave.

\section{Methods}

This retrospective study at the Amsterdam UMC, the Netherlands, included patients admitted to the ICU due to a COVID-19 infection between March and May 2020. Clinical data on patient characteristics, neurological diagnostics, and outcome was collected fro

\section{Results}

Seventy-eight patients (24\% female) were included and median age was 62 (IQR 55-70). ICU mortality was $45 \%$. In 27 patients neurological diagnostics were done; 39 brain CTs, 7 lumbar punctures, 5 brain MRIs and 4 EEGs. Main indication for neurological diagnostics was decreased consciousness (69\%). In 7 patients (26\%) brain abnormalities were detected: infarction, hemorrhage, white matter abnormalities, encephalopathy, focal epilepsy and neuro-inflammation. Median NfL levels at ICU admission for ND+ was $29.2 \mathrm{pg} / \mathrm{ml}$ (IQR 21.2-76.4), for matched-ND- $(\mathrm{n}=23) 29.2 \mathrm{pg} / \mathrm{ml}$ (IQR 19.3-43.4). Median NfL levels at time of neurological diagnostics were $135.9 \mathrm{pg} / \mathrm{ml}$ (IQR 76.6-240.4), for matched-ND- $(\mathrm{n}=22)$ $92.5 \mathrm{pg} / \mathrm{ml}$ (IQR 63.5-136.0). No statistical differences were found.

\section{Conclusions}

Neurological diagnostics were performed in 35\% of ICU COVID-19 patients, abnormalities were found in only $26 \%$. No differences in NfL levels were found between patients with or without neurological diagnostics. 


\section{Poster 112}

\section{Markers of Brain Injury, Endothelial Injury and Inflammation are Elevated after SARS-CoV-2 Infection and are Sex Specific}

H. Alex Choi, Jude Savarraj, Luis Torres, Eunsu Park, Gabriela Colpo, Sarah Hinds, Andres Assing, Diego Morales, Hilda Ahnstedt, Atzhiry Paz, Angela Burkett, Shivanki Juneja, Sung-min Cho, Aaron M. Gusdon, Louise McCullough

UT Health Neuroscience, Houston, TX, United States

\section{Background \& Purpose}

The study of the neurologic effects of SARS-CoV-2 infection is a priority. Sex differences in the immune response to SARS-CoV2 leading to differences in mortality and disease severity of COVID-19 have been reported. To advance our understanding of COVID-19 associated brain injury, we hypothesized that brain injury markers (BIM), endothelial injury markers (EIM) and inflammatory markers (cytokine/chemokines, $\mathrm{CC}$ ) are elevated after COVID-19 and that these elevations are sex specific.

\section{Methods}

Plasma samples from 57 hospitalized COVID-19 subjects, 14 subjects at 3 months after COVID-19 hospitalization and 20 matched controls were used to determine the levels of six BIMs (GFAP, S100B, Sydecan-1, UCHLI, MAP2 and NSE), 38 CCs and two EIMs (sICAM1 and SVCAM1). Statistical and bioinformatics methods were used to measure differences in the marker profiles across (a) COVID-19 vs controls and (b) men vs women.

\section{Results}

Three BIMs: MAP2, NSE and S100B, two EIMs: sICAM1 and sVCAM1 and seven CCs: GRO IL10, sCD40L, IP10, IL1Ra, MCP1 and TNF $\alpha$ were significantly $(p<0.05)$ elevated in the COVID-19 cohort compared to controls. MDC and MIP1 $\alpha$ were significantly lower in the COVID-19 cohort. Bioinformatics analysis revealed a stronger association between BIM/CC/EIMs in the COVID-19 cohort. Analysis across sex revealed that several BIMs and CCs including NSE, IL10, IL15 and IL8 were significantly $(p<0.05)$ higher in men compared to women. Men expressed a more robust BIM/CC/EIM association profile compared to women. At 3 months, the levels of the BIM and CCs were not significantly higher in the COVID-19 cohort compared to controls.

\section{Conclusions}

BIMs, CCs and EIMs were elevated after COVID-19. The robust associations between the BIMs, CCs and EIMs suggest mechanisms of brain injury mediated by inflammation and endothelial disruption. These associations were stronger in men. In a small paired cohort, BMs and CCs returned back to levels of controls at 3 months after COVID-19. 


\title{
Poster 113
}

\section{Clinical Characteristics Associated with Outcomes after Pediatric Catastrophic Brain Injury: A 13-Year Retrospective Cohort Study}

\author{
Kerri L. LaRovere, Bradley J. De Souza, Eliza Szuch, David K. Urion, Sally Vitali, Bo Zhang, Robert J. \\ Graham, Alon Geva, Robert C. Tasker \\ Boston Children's Hospital, Harvard Medical School, Department of Neurology, Boston, MA, United \\ States
}

\section{Background \& Purpose}

In the absence of substantial published data, this study aimed to analyze clinical characteristics associated with outcomes after acute catastrophic brain injury (CBI) in children and adolescents.

\section{Methods}

Single center, 13-year (2008-2020) retrospective cohort study of 106 children in the pediatric and cardiac intensive care units with $\mathrm{CBI}$, defined as 1) new acute neurologic injury based on clinical and imaging findings at presentation; and 2) death or survival with functional neurologic worsening at the time of discharge. The primary outcome of death was related to clinical variables using Kaplan-Meier estimates, unadjusted log-rank test, and multivariable Cox proportional hazard models.

\section{Results}

Of 106 patients (58\% male, median age 3.9 years), 86 (81\%) died. Withdrawal of life-sustaining therapies (WOLST) was most common mode of death (60/86, 70\%). Each unit increase in admission pediatric sequential organ failure assessment (pSOFA) score was associated with $10 \%$ greater hazard of death (HR $1.10,95 \% \mathrm{Cl} 1.04-1.17, \mathrm{p}<0.01)$. Compared with traumatic brain injury (TBI), all other etiologies were associated with greater hazard of death $(p=0.02 ; \mathrm{HR} 3.76-10)$. Median survival time for the cohort was 22 days ( $95 \% \mathrm{Cl} 14-37$ days). Compared to cardiac death mechanisms, patients who died by neurologic criteria had shorter median survival time $(p=0.02)$. Of 23 survivors to hospital discharge, 20 were still alive after a median 2 years (range 1-3 years); 6/20 (30\%) did not have any technology dependence, and $7 / 20$ (35\%) had normal level of consciousness.

\section{Conclusions}

Most children with acute $\mathrm{CBI}$ died within one month of hospitalization. TBI as the etiology of $\mathrm{CBI}$ was associated with improved survival, whereas greater organ dysfunction on admission was associated with increased mortality. Of survivors, many recovered consciousness and did not require permanent technology dependence. These findings may help clinicians guide families with children experiencing $\mathrm{CBI}$. 


\title{
Poster 114
}

\section{A Multi-modal Functional Neuroimaging Approach to Assess Brain Activity Following Cardiac Arrest in the Intensive Care Unit}

Karnig Kazazian, Loretta Norton, Androu Abdalmalak, Teneille E. Gofton, Derek Debicki, Adrian M. Owen

\author{
Western University, Brain and Mind Institute, London, Ontario, Canada
}

\section{Background \& Purpose}

Determining a reliable prognosis for comatose cardiac arrest patients is a substantial clinical challenge. Using advanced neuroimaging methods such as functional Magnetic Resonance Imaging (fMRI) and functional Near Infrared Spectroscopy (fNIRS) while simultaneously stimulating the median nerve may provide insight into the integrity of higher-order brain areas following injury. The extent of neural activity in response to this task may be indicative of preserved cortical function and ultimate neurologic outcome.

\section{Methods}

To date, 25 healthy controls with no history of brain injury have been recruited for the fMRI task, of which 10 have also participated in the fNIRS task. Median nerve stimulation was performed simultaneously with neuroimaging testing with the same clinical parameters used in upper limb somatosensory evoked potentials. The right and left hand was stimulated successively at a current that sustained thumb abduction (8-35 mA). A block design with eight cycles of stimulation 'on' and seven cycles of 'rest' was used. The data was analyzed at a statistical threshold of $p<0.05$, family-wise error corrected for multiple comparisons. Recruitment of patients has not started due to on-going COVID-19 restrictions.

\section{Results}

fMRI results display significant activity in the contralateral primary somatosensory cortex, bilateral secondary somatosensory cortex, and bilateral insula for each hand being stimulated at the group level. fNIRS results show significant activity in the contralateral primary somatosensory cortex and bilateral secondary somatosensory cortex. We found an $80 \%$ agreement between fMRI and fNIRS results over these regions of interest.

\section{Conclusions}

This pilot study is the first step towards establishing objective and quantifiable neural markers of good neurological outcome in the ICU. Neural activity recorded from healthy controls will serve as a reference for patients enrolled in this study. Moving forward, this study will recruit acute comatose cardiac arrest patients to assess the prognostic utility of fMRI and fNIRS with this paradigm. 


\section{Poster 115}

\section{Brain Death after Stroke: Factors Affecting Organ Donation}

P. Varelas, M. Rehman, M. Kananeh, P. Brady, L. Louchard, C. Mehta, T. Abdelhak, P. Mitsias

Albany medical College, Department of Neurology, Albany, NY, United States

\section{Background \& Purpose}

Patients with stroke have a $24-48 \%$ chance to be declared brain dead (BD), with highest probability after subarachnoid hemorrhage (SAH), followed by intracerebral hemorrhage (ICH) and lowest after ischemic stroke (IS). No information exists on variables associated with organ donation in these patients.

\section{Methods}

Between 2006 and 2017 all patients who became BD at Henry Ford Hospital were entered in a database. Only those patients with stroke in this database were included in the analysis.

\section{Results}

128 stroke patients were declared BD [mean age $56 \pm 13.5$ years, $43.8 \%$ males, $34.2 \%$ Caucasian, $59.2 \%$ African Americans (AAs), 31 (24\%) with IS, 75 (59\%) ICH and 22 (17\%) SAH]. Families of 106 patients were approached and 75 (70.8\%) consented for organ donation. There was no difference in consent based on age, sex, religion, organ function at the time of death, apnea testing, confirmatory tests, timing from event to declaration of $\mathrm{BD}$, or number of clinical exams. In the univariate analysis, $61.4 \%$ of AAs families vs $88.6 \%$ of non-AAs (chi-square $p=0.005$ ) and $88.2 \%$ of SAH families vs $67.4 \%$ of other types of stroke consented $(p=0.08)$. However, in a logistic regression adjusting for first-person consent, age, and type of stroke, only AAs showed a significant association with organ donation consent (odds ratio, $95 \%$ $\mathrm{Cl} 0.2,0.06-0.67, \mathrm{p}=0.009$ ). There was no difference in the number of organs recovered or transplanted across stroke types and race categorization (ANOVA, $p>0.1$ ).

\section{Conclusions}

AAs, who present with stroke and progress to become BD, have 5 times lower consent rate for organ donation compared to non-AAs. Because this population subgroup is in higher need for organ transplantation, our finding warrants further study. 


\section{Poster 116}

\section{Brain Injury Monitoring Post-cardiac Arrest Using Non-invasive Cranial Accelerometry}

Rachel Muster, Jonathan Shih, Maximilian Vuong, Dominica Randazzo, Edilberto Amorim, Wade Smith

University of California, San Francisco, Department of Neurology, San Francisco, CA, United States

\section{Background \& Purpose}

Hypoxic-ischemic brain injury after cardiac arrest (CA) can cause brain edema and elevated intracranial pressure. The lack of non-invasive measures of early changes in brain tissue post-CA has limited the identification of patients with potential to benefit from early interventions. The headpulse is a measure of imperceptible skull movements during the cardiac cycle that can be measured using non-invasive 3axis accelerometers placed on the temples. This headpulse waveform has been shown to be abnormal in patients with neurological impairment from acute stroke, concussion, and vasospasm following aneurysmal subarachnoid hemorrhage, however its role in brain injury risk stratification in CA is unknown.

\section{Methods}

We compared the frequency spectra of the headpulse (25th, 50th, 75th, and 90th percentiles by area) in 18 post-CA subjects ( $\mathrm{N}=73$ total serial measurements over 286 hours since return of spontaneous circulation) and 10 control subjects. Subgroup analyses of CA subjects with poor neurological outcomes (Cerebral Performance Category [CPC] 3-5) at discharge, brain edema detected on head CT or MRI, and unfavorable EEG (i.e. epileptiform or burst suppression patterns) were pursued.

\section{Results}

CA subjects had lower frequency spectra compared to controls ( $p<0.05$ for all frequency percentiles in anterior-posterior and lateral axes and 50-90th percentiles in vertical axis). In the first $48 \mathrm{~h}$ post-CA, CA subjects with poor neurological outcomes ( $N=12,11$ recordings) had a trend toward lower frequency spectra in the lateral and vertical axes and significantly lower frequency spectra in the 90th percentile of the vertical axis than subjects with good outcomes $(N=5,6$ recordings, $p<0.05)$. Among CA subjects, those diagnosed with brain edema $(\mathrm{N}=7)$ on imaging or unfavorable $E E G(N=12)$ had lower frequency spectra at the 90th percentile in the vertical axis $(p<0.05)$.

\section{Conclusions}

Headpulse frequency spectra is abnormal in post-CA subjects and may support brain injury risk stratification and predict neurological outcomes post-CA. 


\title{
Poster 117
}

\section{Characteristics of Progressive Hypoxic Ischemic Brain Injury On Head Computed Tomography In Comatose Survivors Post-Cardiac Arrest}

\author{
Matthew Du, Faten El Ammar, Jared Davis, Ali Mansour, Fernando D. Goldenberg \\ University of Chicago Pritzker School of Medicine, Chicago, IL, United States
}

\section{Background \& Purpose}

The identification of hypoxic-ischemic brain injury (HIBI) on head computed tomography (HCT) has grown in promise as a radiologic prognosticator of neurologic outcome post cardiac arrest. This study assesses the prognostic value of serial HCT imaging on comatose survivors of cardiac arrest.

\section{Methods}

Retrospective review of demographic, clinical, and radiographic information of 140 comatose survivors admitted at an academic medical center from 2017 to 2020 . HCT scans were reviewed by a board certified neuroradiologist and were categorized as "normal" and "abnormal" based on the absence or presence of $\mathrm{HIBI}$, respectively. Subjects with an initial scan (performed mostly within 3 hours of Return of Spontaneous Circulation - ROSC) categorized as normal and a repeat scan (performed mostly within 2 to 5 days after ROSC) that was abnormal or normal were considered as showing and not showing $\mathrm{HIBI}$ progression, respectively.

\section{Results}

Among 101 subjects who expired, those with an initial abnormal HCT were more likely to evolve to brain death $(44.4 \%)$ than those with a normal HCT $(6.2 \%)(p<0.001)$. Furthermore, those with an initial abnormal HCT also had a higher chance of evolving to brain death than those who showed HIBI progression on the 2 nd HCT (44.4\% versus $14.3 \%, p=0.041$ ). Among fifty-eight subjects who had an initial normal HCT, twenty-nine (50.0\%) showed HIBI progression on the repeat HCT. HIBI progression was significantly associated with absent corneal reflex on initial exam $(\mathrm{OR}=3.82,95 \% \mathrm{Cl}=1.16-13.63, \mathrm{P}=0.031)$ but not with a higher likelihood of mortality during admission ( $\mathrm{OR}=1.97,95 \% \mathrm{Cl}=0.47-7.07, \mathrm{P}=0.283$ ).

\section{Conclusions}

Presence of HIBI on early HCT after ROSC is associated with evolution to brain death in $44 \%$ of the cases despite best contemporary clinical care. Progression of HIBI from 1st to 2nd HCT didn't increase the likelihood of in-hospital death in our group. Further research on temporal evolution of HIBI on HCT should be undertaken. 


\section{Poster 118}

\section{Decision Tool for Identifying out of Hospital Cardiac Arrest Patients at High Risk for Brain Death}

Patrick J. Coppler, Clifton W. Callaway, Jonathan Elmer

University of Pittsburgh Department of Emergency Medicine, Pittsburgh PA, United States

\section{Background \& Purpose}

Patients with return of spontaneous circulation after out of hospital cardiac arrest (OHCA) are often comatose secondary to anoxic brain injury. Some progress to brain death. No validated decision rules identify patients at high risk of brain death. We hypothesized arrest characteristics, qualitative and quantitative assessments of cerebral edema on presenting brain computed tomography (CT) and initial electroencephalography (EEG) predict brain death.

\section{Methods}

We identified comatose OHCA patients from our prospective registry who presented between January 2010 to July 2019. We abstracted age, sex, arrest rhythm, arrest etiology, CPR duration, epinephrine doses, number of shocks; Pittsburgh Cardiac Arrest Category, initial motor exam and pupillary light reflex; brain CT grey-to-white ratio (GWR), sulcal effacement, and effacement of the basal cisterns; initial EEG background and suppression ratio. We used multiple imputation with chained equations to handle missing data. We estimated models using a bootstrap, iteratively randomly partitioning cases into $80 \%$ training and $20 \%$ test sets. We used Fast and frugal tree (FFT) analysis, an approach that creates parsimonious, interpretable decision rules. We compared performance to a saturated multivariable logistic regression. We determined mean sensitivity and specificity at Youden's J-point for each approach.

\section{Results}

We included 1566 patients, of whom 146 were pronounced brain dead. Across bootstrapped samples, $>99 \%$ of FFTs 3 predictors of progression to brain death: absence of sulci, and in cases without sulcal effacement, the combination of EEG background suppression and GWR $<=1.23$. Predicting brain death, these had mean sensitivity and specificity of $87 \%$ and $81 \%$, respectively. Logistic regression with all available predictors had incrementally better performance ( $91 \%$ sensitivity, $83 \%$ specificity) at the expense of a much more complex model.

\section{Conclusions}

Initial loss of sulci on brain CT or absence of EEG background with GWR $<1.23$ accurately predicts progression to brain death. 


\section{Poster 119}

\section{Disorders of Consciousness Associated with COVID-19: A Prospective, Multimodal Study of Recovery and Brain Connectivity}

David Fischer, Samuel B. Snider, Megan E. Barra, William Sanders, Otto Rapalino, Pamela Schaefer, Yelena G. Bodien, Brian L. Edlow

Massachusetts General Hospital, Neurology Department, Division of Neurocritical Care, Boston, MA, United States

\section{Background \& Purpose}

In patients with severe coronavirus disease 2019 (COVID-19), disorders of consciousness (COVID-DoC) have emerged as a complication with an unclear prognosis and pathophysiology. We describe the natural history of COVID-DoC and investigate its associated brain connectivity profile.

\section{Methods}

In a prospective, longitudinal study, we screened consecutive inpatients with COVID-19 at our institution between July 2020 and March 2021. We enrolled adult patients who were admitted to an intensive care unit, had a disorder of consciousness unexplained by sedation or structural brain injury, and were planned to undergo a brain MRI. We performed resting state functional MRI and diffusion MRI to evaluate functional and structural connectivity, as compared to healthy controls and patients with disorders of consciousness resulting from severe traumatic brain injury (TBI). We assessed the recovery of consciousness (command-following) and functional outcomes (Glasgow Outcome Scale Extended [GOSE] and the Disability Rating Scale [DRS]) at hospital discharge, three months post-discharge, and six months post-discharge.

\section{Results}

After screening 1,105 patients with COVID-19, we enrolled twelve with COVID-DoC. The median age was 63.5 years [interquartile range 55-76.3]. Excluding one who died shortly after enrollment, all of the remaining eleven patients recovered consciousness, after a median of seven days [5-14.5, range 0-25] following the cessation of continuous sedation. At discharge, the median GOSE score was 3 [1-3], and the median DRS was 23 [16.25-30]; at three months post-discharge, the median GOSE score was 3 [3-3], and the median DRS was 9 [5-15.5]. Two patients evaluated at six months post-discharge had minimal disability. Brain connectivity in COVID-DoC was diminished compared to healthy controls, and comparable to patients with severe TBI.

\section{Conclusions}

Patients who survived COVID-19 invariably recovered consciousness after COVID-DoC. Disability, though common following hospitalization, improved over the ensuing months. While future research is necessary, these prospective findings begin to inform the prognosis and pathophysiology of COVID-DoC. 


\title{
Poster 120
}

\section{EEG/ECoG Metrics Near-death during Cardiac Arrest Elucidate Neurovascular Coupling and Correlate with Neurological Recovery Post-resuscitation}

\author{
Justin Yi, Samuel Tincher, Sangwoo Han, Afsheen Bazrafkan, Masih Rafi, Shirin Mansooridara, Anais \\ Panossian, Alyssa Avilez, Ashwin Nath, Haein Yun, Kieran S. McCluskey, Yama Akbari, Robert H. Wilson \\ University of California, Irvine, School of Medicine, Irvine, CA, United States
}

\section{Background \& Purpose}

Cerebral anoxia during cardiac arrest (CA) can cause profound neurological disability. Neurological recovery depends on functional restoration of circuits which suffer rapid synaptic failure post-CA. Synaptic dysfunction and recovery can be characterized via frequency-domain analysis of electrocorticography (ECoG).

\section{Methods}

Using our preclinical model mimicking a neuro-intensive care unit, 10 male Wistar rats underwent asphyxial CA (5 or $7 \mathrm{~min}$ ) and successful CPR. In 5-second windows, the ECoG spectrum was analyzed to quantify temporal changes in the relative attenuation of successively higher frequencies, the " $1 / \mathrm{f}$ slope," a measure of synaptic failure and recovery. ECoG burst suppression ratio (BSR) up to 80 min postresuscitation was used as a surrogate marker of neurological recovery.

\section{Results}

Before asphyxia, the mean 1/f slope was $-3.7 \mathrm{~Hz}-1$, but $\sim 40 \mathrm{~s}$ after asphyxia, it rapidly increased to -1.0 $\mathrm{Hz}-1$. After resuscitation, the $1 / \mathrm{f}$ slope decayed back to baseline. The mean decay rate of $1 / \mathrm{f}$ slope following CPR was inversely proportional to the duration of the "AS3" period during entry into CA ( $r=$ $0.93, p=0.0027,1$ outlier removed), a period we have previously identified increased functional connectivity. The early $1 / \mathrm{f}$ decay rate post-CPR was also proportional to mean change in BSR later (40-80 minutes) post-CPR ( $r=0.86, p=0.0063) .5$ min CA rats had significantly faster decay rate than 7 min CA rats $(p=0.0045)$.

\section{Conclusions}

The ECoG $1 /$ f slope quantifies dysfunction and recovery of neural circuits. The $1 / f$ slope jumps rapidly during electrocerebral silence and subsequently decays slowly post-CPR. A faster $1 / f$ slope decay rate correlated with shorter CA and lower BSR 40-80 min post-CA, suggesting faster return to sustained neural activity. Faster $1 / f$ slope decay rate was also associated with a longer period of anoxia-induced functional connectivity during entry into $C A$, perhaps suggesting that the baseline robustness of synaptic connections influences post-CPR recovery. 


\section{Poster 121}

\section{Impact of Opioids and Sedatives on Time to Recovery of Consciousness After Intracerebral Hemorrhage}

Caroline Der-Nigoghossian, Ayham Alkhachroum, Qi Shen, Kevin Doyle, Jennifer Egbebike, Jon Rosenberg, Lucy Panicker, Greer Waldrop, Jeremy Ader, Lauren H. Grobois, Athina Vrosgou, David Roh, Soojin Park, Sachin Agarwal, E Sander Connolly, Jan Claassen

Department of Pharmacy, NewYork-Presbyterian Hospital, Columbia University Irving Medical Center, New York, NY, United States

\section{Background \& Purpose}

It is uncertain how opioids and sedatives affect time to recovery of consciousness (ROC) in unresponsive intracerebral hemorrhage $(\mathrm{ICH})$ patients managed with a light sedation protocol including daily sedative interruptions. We aimed to characterize the impact of opioids and sedatives in the context of other predictors of time to ROC prior to hospital discharge.

\section{Methods}

In this single-center retrospective observational cohort study, we investigated adult ICH patients who were admitted to the neurological ICU between 02/2009 and 01/2018. We included patients who did not follow at least simple commands by day 2 of ICU admission. ROC was assessed daily and defined as the ability to follow at least simple commands. A cox proportional hazards model was used to evaluate the effect of opioids and sedatives on the time to ROC during hospitalization.

\section{Results}

From $157 \mathrm{ICH}$ patients, 63 recovered consciousness during the hospital admission. Mean time to recovery of consciousness was $9.9+/-13.5$ days. Patients who did not recover were older (70 vs 63 years old, $p=0.045)$, had higher incidence of intraventricular hemorrhage $(74 \%$ vs $56 \%, p=0.014)$, ICH volume $>30 \mathrm{~mL}$ (66\% vs 30\%, p < 0.001), and higher cumulative opioid equivalents ( 0.9 vs $1.8 \mathrm{mg}, \mathrm{p}=0.035)$. In our cox proportional hazards model, ICH volume $>30 \mathrm{~mL}$ (HR 0.30; 95\% Cl 0.19-0.47), presence of intraventricular hemorrhage ( $\mathrm{HR} 0.42 ; 95 \% \mathrm{Cl} 0.26-0.66)$, and age $>80$ y.o. ( $\mathrm{HR} 0.43 ; 95 \% \mathrm{Cl} 0.22-0.86$ ) prolonged the time to ROC. Cumulative opioids or sedatives were not associated with time to ROC.

\section{Conclusions}

Components of the primary ICH score, which was developed to predict mortality at 30 days, predict inhospital time to $\mathrm{ROC}$ in $\mathrm{ICH}$ patients managed with a light sedation protocol that includes daily sedative interruptions. In this context, opiates and sedatives do not prolong time to ROC. 


\title{
Poster 123
}

\section{Ketamine Administration without Coma Induction in the Emergency Setting}

\author{
Megan E. Barra, Guneeti Sharma, James C. Cloyd, Lisa D. Coles Can Ozan Tan, Joshua N. Goldstein, Bryan \\ D. Hayes, Jaideep Kapur, James M. Chamberlain, Robert Silbergleit, MD(8); Thomas P. Bleck, Andrew \\ Cole, Eric S. Rosenthal
}

Department of Pharmacy, Massachusetts General Hospital, Boston, MA, United States

\section{Background \& Purpose}

Ketamine has been proposed as an intubation-sparing therapy to terminate established status epilepticus (SE). Because persistent coma is historically considered treatment failure, we sought to examine ketamine's effect on neurologic function using time-synchronized electronic health record data.

\section{Methods}

We retrospectively studied adults administered parenteral ketamine bolus in the emergency department (04/2016-05/2020) with Glasgow Coma Scale (GCS) score documented <24 hours pre- and post-administration. Change in GCS $(\triangle \mathrm{GCS}) \leq 4$ hours post-administration and incidence of unintended intubation were evaluated.

\section{Results}

122 patients met inclusion. Indications for ketamine administration included planned intubation (61.5\%), agitation (22.1\%), and procedural sedation (13.1\%). Median age was 55 years [IQR 36, 68]; average dose was $1.3 \mathrm{mg} / \mathrm{kg}$ (SD 0.8); and routes were intravenous (86.1\%) or intramuscular (13.9\%). When administered for indications other than planned intubation $(n=47)$, median post-ketamine $\Delta G C S$ was $0[-1,2]$ at $0-1.9$ hours $(n=13)$ and $0[0,0]$ at $2.0-3.9$ hours $(n=14) .15 .4 \%(2 / 13)$ of patients experienced a GCS decline $\geq 2$ at 0-1.9 hours and 0\% at 2-3.9 hours. When administered for planned intubation ( $n=75)$, median post-ketamine $\triangle$ GCS was $-3[-7,0]$ at $0-1.9$ hours $(n=17)$ and $-7[-10,-3]$ at 2.03.9 hours $(n=31) .64 .7 \%(11 / 17)$ experienced a GCS decline $\geq 2$ at $0-1.9$ hour and $77.4 \%(24 / 31)$ at 2-3.9 hours. No differences were observed when stratifying by dose $(\geq 1.5 \mathrm{mg} / \mathrm{kg}$ versus $<1.5 \mathrm{mg} / \mathrm{kg}$ ) or age ( $\geq 65$ versus $<65$ years). Unintended intubation occurred in $8.5 \%(4 / 47)$, all unrelated to ketamine adverse effects, attributed instead to inadequate response $(n=2)$, metabolic disarray-induced respiratory depression $(n=1)$, and surgical intervention $(n=1)$.

\section{Conclusions}

Ketamine bolused for agitation or procedural sedation did not routinely induce coma. GCS deterioration $\geq 2$ points was uncommon, and events of unintended intubation were not attributable to adverse effects. These findings may guide future trials in SE patients, for whom coma recovery and intubation are constituent clinical outcomes. 


\section{Poster 124}

\section{Outcomes, Time-Trends, and Factors Associated with Ancillary Study Use for the Determination of Brain Death}

Ibrahim Migdady, Moein Amin, Aaron Shoskes, Catherine Hassett, Alexander Rae-Grant, Sam B. Snider, David M. Greer, Sung-Min Cho, Pravin George

Massachusetts General Hospital, Department of Neurology, Boston, MA, United States

\section{Background \& Purpose}

Brain death (BD) determination often requires ancillary studies when clinical determination cannot be completed. We aimed to analyze the results of ancillary studies, factors associated with ancillary study performance, and changes in number of studies performed over time at an academic health system.

\section{Methods}

In retrospective cohort, we compared characteristics of patients who underwent ancillary studies for BD with those who did not. Linear and logistic regression analyses were used to identify time trends and factors associated with ancillary study use. Odds ratios (OR) and 95\% confidence intervals (Cl) were calculated.

\section{Results}

Of 140 BD patients, ancillary studies were performed in 84 (60\%). The false negative rate of all studies was $4 \%$. In univariate analysis, ancillary study use was associated with female sex (OR 2.4; $\mathrm{Cl} 1.21-5.01$; $\mathrm{p}=0.013)$ and the etiology of brain death being hypoxic-ischemic brain injury (OR, 2.9; $\mathrm{Cl} 1.43-5.88 ; \mathrm{p}=$ $0.003)$, spontaneous intracranial hemorrhage $(\mathrm{OR}, 0.45 ; \mathrm{Cl}, 0.21-0.96 ; \mathrm{p}=0.039)$, or traumatic brain injury (OR, 0.22; $\mathrm{Cl} 0.04-0.8 ; \mathrm{p}=0.031)$. In multivariable analysis, female sex (OR, 5.7; $\mathrm{Cl} 2.56-15.86 ; \mathrm{p}=$ $0.004)$, the etiology of BD being hypoxic- ischemic brain injury (OR, 3.2; $\mathrm{Cl} 1.3-8.8 ; \mathrm{p}=0.015)$, and neurologists performing $B D$ assessment $(O R, 0.08 ; \mathrm{Cl} 0.004-0.64 ; p=0.034$ ) were factors independently associated with ancillary study use. Only the number of electroencephalograms utilized significantly changed with time (OR per 1-yr increase, 0.67; $\mathrm{Cl} 0.49-0.90 ; \mathrm{p}=0.014$ ).

\section{Conclusions}

A large number of ancillary studies were performed despite a clinical determination of BD; patients with $\mathrm{HIBI}$ were more likely to undergo ancillary studies, and neurologists were less likely to use ancillary studies for BD determination. Recently, the use of electroencephalograms for BD determination has decreased, likely reflecting significant concerns regarding its validity and reliability. 
Poster 126

\title{
Retrospective Chart Review on Declaration of Brain Death Across a Hospital System
}

\author{
Stacey Williamson, Kristine O'Phelan
}

Jackson Memorial Hospital, University of Miami Department of Neurology, Miami, FL, United States

\section{Background \& Purpose}

Patients who suffer from a devastating neurologic injury such as intracranial hemorrhage, malignant ischemic stroke, or cardiac arrest resulting in anoxic brain injury will often progress to brain death when their original insult is very severe. Specific requirements for determining Brain Death not only vary by state but also across hospital systems. This variability can lead to inaccurate determination of Brain Death. This may be more common when not all prerequisite criteria in the AAN guidelines are met. This review serves to determine if Brain Death declarations performed within a hospital system consistently meet AAN Guidelines for determining brain death.

\section{Methods}

Retrospective chart review of 1307 patients referred to Life Alliance for Organ Donation with evidence of severe brain injury defined as GCS $<6$ and ventilated over a 12-month time period.

\section{Results}

Out of 1307 patients, 61 were declared brain dead. 37 (60.6\%) fulfilled prerequisite and diagnostic guidelines as set by AAN and the state of Florida for determination of brain death. Of the 24 (39.3\%) who did not, the most common omissions were: absence of second physician exam $22.9 \%$ (14) presence of underlying metabolic derangements $13.1 \%$ (8), and hypothermia $8.2 \%$ (5).

\section{Conclusions}

Variations in provider specialty (Trauma vs Pulmonary-Critical Care), hospital unit (Surgical vs Medical ICU), and patient not meeting prerequisites for determining brain death result in patients not fulfilling AAN recommendations for determination of Brain Death. Consistency in accurate declaration and documentation of Brain Death when prerequisite criteria met improved to 25 of 37 (67.6\%) when either Neurology or Neurosurgery were the primary team and patient was admitted to the Neuroscience ICU. Implementation of a standardized method for Brain Death determination including meeting prerequisite criteria, proper documentation, checklists, and provider education may result in more accurate and timely declaration across the hospital system. 


\section{Poster 127}

\section{Simultaneous Subgaleal Electrode and Standard Scalp Long Term cEEG Monitoring Following Cardiac Arrest: A Prospective Comparative Study}

Laith Altaweel, Mohan Kurukumbi, Leonard Genovese, Yun Fang, Jing Wang, Hussain Dhanani, Asma Zakaria, Saqib Chaudhry, Ron Emerson, Shashank S. Sinha, Allen Waziri

Inova Department of Medicine and Neurociences, Falls Church, VA, United States

\section{Background \& Purpose}

Long-term EEG monitoring in cardiac arrest (CA) patients is critical for neuroprognostication and detection of seizures. However, conventional scalp EEG (SC) signal quality is highly dependent on frequent technical maintenance. Subgaleal (SG) electrodes may provide an alternative means of acquiring high-quality cEEG with minimal technical support.

\section{Methods}

Single-center, prospective comparison of simultaneous SGEEG and SCEEG in comatose CA patients admitted to the $\mathrm{CICU}$ undergoing targeted temperature management. Standard SC electrodes were placed and maintained twice daily by a technician. Bihemispheric 8-channel parasagittal SG electrodes were placed by study investigators using a trocar-and-sheath device. Matched two-minute SCEEG and SGEEG epochs, assessed at hourly intervals for up to 12 hours during hypothermia, rewarming and normothermia, were independently scored for signal quality and noise level using a previously described scale (from 1-5; higher score indicating better quality) by two electrophysiologists.

\section{Results}

Seven patients (ages 45-83 years) were enrolled; 4 with initial shockable rhythms. Mean (range) GCS was 4.3 (3-9). 1 patient survived to discharge. Mean (range) duration of simultaneous SG and SC cEEG monitoring was 101 hours (39-210). There were no study-based adverse events. 892 quality measurements demonstrated interobserver kappa from 0.41 to 0.54 . Mean (SD) scores were comparable during hypothermia $(p=0.83$ ) but favored SG over SC during rewarming (signal: $5.0+0.2$ vs $4.6+0.7$; noise $4.9+0.3$ vs $4.5+0.7$; both $p<0.001$ ) and normothermia (signal: $4.7+0.6$ vs $4.1+1.1$; noise: $4.5+0.7$ vs $3.9+1.1$; both $p<0.001$ ).

\section{Conclusions}

Long-term SGEEG, as compared to SCEEG, provides safe, durable, and high-quality monitoring for comatose CA patients. In this small cohort, SGEEG was superior to SCEEG during periods of rewarming and normothermia due to greater signal-to-noise ratio. 


\section{Poster 128}

\section{Soluble CD73 Levels Correlate with Cerebral Edema Post Cardiac Arrest}

Jonathan Rud, Richard Riker, Andrew Bradbury, Joanne deKay, David Pozivilko, Ashley Eldridge, Michael DiGianvittorio, Teresa May, Sergey Ryzhov, David Seder

Maine Medical Center, Portland, ME, United States

\section{Background \& Purpose}

Early cerebral edema after out of hospital cardiac arrest is associated with poor outcomes, the pathophysiology of which is not well understood. This study evaluated the association of soluble CD73, an immunomodulatory protein known to influence vascular permeability, with cerebral edema after cardiac arrest.

\section{Methods}

After surrogate consent, 40 out of hospital cardiac arrest patients had serial blood samples obtained at predetermined intervals. Circulating soluble CD73 was measured using commercially available enzyme linked immunosorbent assay (ELISA). CT imaging of the head obtained during the first $24 \mathrm{hr}$ post resuscitation was reviewed and scored for gray-white ratio (GWR) by a board certified neuroradiologist blinded to clinical and sCD73 data. Correlation was evaluated between GWR and CD73 levels at 6, 12, and $24 \mathrm{hrs}$ post resuscitation.

\section{Results}

The mean age of study subjects was 62 years of age, $26 \%$ were female, $63 \%$ witnessed event, $33 \%$ received bystander $\mathrm{CPR}, 59 \%$ had shockable rhythms and mean ischemic time was 15.9 minutes \pm 13.0 minutes. 26 patients had CT scans available. Soluble CD73 was inversely correlated with GWR ratio at $12 \mathrm{hr}(\mathrm{p}=.034 \mathrm{R} 2=.18)$ and $24 \mathrm{hrs}(\mathrm{p}=.037 \mathrm{R} 2=.18)$

\section{Conclusions}

Levels of circulating soluble CD73 following resuscitation from out of hospital cardiac arrest is inversely correlated with GWR. The association between this vasoregulatory molecule with cerebral edema after cardiac arrest warrants further characterization. 


\section{Poster 129}

The Accuracy of Coma Prediction Improves during the First Three days of Neurocritical Care and is Influenced by the Severity and Persistence of Neurologic Deterioration

Eric S. Rosenthal, Guneeti Sharma, Sai Nooney, Sophie Ack, India A. Lissak, Craig Maddux, Dick Moberg, Xiao Hu, J. Claude Hemphill, Saef Izzy, Edilberto Amorim, Brandon Foreman

Department of Neurology, Massachusetts General Hospital, Boston, MA, United States

\section{Background \& Purpose}

We sought to quantify how prognostic accuracy changes over the first $72 \mathrm{~h}$ of neurocritical care. We developed admission and serial progress models predicting discharge Glasgow Coma Scale score using electronic health record (EHR) data. Covariates included severity and duration of neurologic deterioration (ND).

\section{Methods}

We analyzed consecutive admissions to four neurocritical care units within the Collaborative Hospital Repository to Unlock Synergy (CHoRUS) workgroup, excluding patients <18 years or without GCS score documentation within $48 \mathrm{~h}$ of discharge. Utilizing age, day 1 ICD-10 codes, and initial total GCS (iGCS) score, we developed a baseline Random Forest model predicting the final GCS score (+/-1 point). We then developed three progress models, adding features from GCS data documented during the initial 24, 48 , or $72 \mathrm{~h}$. GCS features included minimum, maximum, mean, median, variance, maximum, ND severity, and ND duration. Model training utilized 10 -fold cross-validation on $70 \%$ of data; accuracy $[95 \% \mathrm{Cl}]$ is reported for the $30 \%$-holdout testing cohort for each model. Feature importance (mean decrease in accuracy) is reported for the 72-h model.

\section{Results}

24,204 unique admissions met inclusion criteria (mean age 57.6-61.6 years across sites). iGCS score was median [IQR] 15 [11,15]. 45\% had a ND >2 points; $29 \%$ had a ND >4 points. 2-point deteriorations lasted a mean (SD) of 27.9h (43.1); 4-point deteriorations lasted 46.4h (78.3). Model accuracy increased from 0.64 [0.61-0.66] (baseline) to 0.70 [0.68-0.73] (24h), 0.73 [0.70-0.75] (48h), and 0.76 [0.74-0.79] (72h). Feature importance at $72 \mathrm{~h}$ was greatest for ND-max (77), followed by ND-tmax (70), but only 35 for iGCS.

\section{Conclusions}

Among neurocritical care patients, predicting discharge GCS score was increasingly accurate over the initial 72h. Initial GCS score had low feature importance compared to the severity and duration of ND events. Future directions include disease-specific prediction and assessing the importance of salient events (neuroimaging or neurosurgery) co-occurring with events of neurologic deterioration. 


\title{
Poster 130
}

\section{The Influence of Race and Ethnicity on Cardiac Arrest Outcomes}

\author{
Gabriella E. Garcia, Nidhi Ravishankar, Emily Gilmore, Rachel Beekman
}

Yale University, Department of Neurology, New Haven, CT, United States

\section{Background \& Purpose}

Racial and ethnic disparities exist in cardiac arrest outcomes. We sought to evaluate these disparities by analyzing pre- and in-hospital variables available in our cardiac arrest database.

\section{Methods}

We performed a retrospective review of in-hospital and out-of-hospital cardiac arrests between 20112017 at a single academic center. Pearson chi-squared and linear regression were used to compare variables.

\section{Results}

Of 258 cardiac arrest patients, 5 were excluded due to missing race data. 163 (64.4\%) identified as nonHispanic Caucasian (W), 59 (23.3\%) identified as non-Hispanic Black (B), and 31 (12.2\%) identified as Hispanic $(H)$. Minority patients were younger ( $\mathrm{B}: \mathrm{N}=54(17.3 \%)$ and $\mathrm{H}: \mathrm{N}=50(16.1 \%)$ years vs $\mathrm{W}: \mathrm{N}=60$ (16.6\%) years, $\mathrm{P}=0.002)$ and more likely to have end-stage renal disease (ESRD) $(\mathrm{B}: 16.9 \%$ and $\mathrm{H}: 20.7 \%$ vs. $\mathrm{W}: 5.5 \%, \mathrm{P}=0.005)$. Hispanic patients were less likely to receive by-stander $\mathrm{CPR}(\mathrm{H}: 32.3 \% \mathrm{vs}$. $\mathrm{W}: 60.1 \%$ and $\mathrm{B}: 61.0 \%, \mathrm{P}=0.012)$. There were no differences in survival to discharge or good neurologic outcomes [W:25.8\% vs. B:30.5\% vs. $\mathrm{H}: 22.6 \%, \mathrm{P}=0.678$ and $\mathrm{W}: 10.4 \%$ vs. $\mathrm{B}: 6.8 \%$ vs. $\mathrm{H}: 6.5 \%, \mathrm{P}=0.608$, respectively]. In Black patients, withdrawal of life sustaining therapy (WLST) was less frequent and time to WLST was longer [B:40.7\% vs W:66.7\% and $\mathrm{H}: 61.3 \%, \mathrm{P}=0.002$ and $\mathrm{B}: 7$ [IQR 5-15] vs. W:4 [IQR 1-7] and $\mathrm{H}: 6$ [ IQR 16 ], $P<0.001$, respectively]. After adjustment for age, sex, location of arrest, bystander CPR, and rhythm, Black patients were more likely to progress to brain death [OR 3.38 (1.04-11.75)].

\section{Conclusions}

Black and Hispanic patients presented with cardiac arrest at a younger age and had higher rates of ESRD. Black race was an independent predictor of brain death. This may be due to delayed WLST in Blacks patients, as earlier WLST in non-Black patients may have prohibited brain death determination. However, these findings need to be validated in similar mixed-race cohorts to identify modifiable risk factors that could impact outcome. 


\section{Poster 131}

\section{"We Don't Have a Crystal Ball": A Multicenter Study of the Words Clinicians use to Disclose Prognosis to Families of Patients with Critical Neurologic Illness}

Adeline L. Goss, Connie Ge, Sybil Crawford

UCSF Neurology, San Francisco, CA, United States

\section{Background \& Purpose}

Communicating the prognosis of critically ill neurologic patients (CINPs) to their families is among the most difficult, yet important, tasks for clinicians. Little is known about how clinicians actually disclose prognosis in CINPs or what language they use.

\section{Methods}

We audio-recorded and analyzed 43 clinician-family meetings for 39 CINPs with 88 family members and 27 clinicians at seven U.S. centers. Using an existing framework, two investigators qualitatively coded prognostic statements as probabilistic (estimating the likelihood of an outcome, e.g." $30 \%$ of patients die") or non-probabilistic (characterizing outcomes without mentioning likelihood, e.g. "We don't have much hope"). We employed multivariable negative binomial regression with a random clinician effect to identify factors associated with more prognostication.

\section{Results}

We identified 512 unique prognostic statements (median 9/meeting [IQR 4;18]), of which 196 (38\%) were probabilistic and 316 (62\%) non-probabilistic. Survival was seldom discussed as an outcome (median number of statements per meeting 0 [IQR 0;2]), while physical function and cognition were most commonly discussed (median 2 [IQR 0;7] and 2 [IQR 0;6], respectively). Clinician specialty (surgery $>$ neurology>internal medicine; $p=0.007$ ) was independently associated with more prognostic statements, as was higher clinician training level (attending vs. other; $p<0.0001)$, fewer years in practice (1st tertile [8years] $>3$ rd tertile [19years]; $p=0.009$, lower patient age (1st tertile [50years] $>3$ rd tertile [78years]; $<<0.0001$ ), neurologic diagnosis (other>hemorrhagic stroke $>$ ischemic stroke $>T B I ; p=0.03$ ), and meeting length (1st tertile [19min] $>3$ rd tertile $[41 \mathrm{~min}] ; p<0.0001)$. For probabilistic and non-probabilistic statements separately, the same predictors persisted with a similar magnitude of association, except that for probabilistic statements, clinicians' years in practice and neurologic diagnosis were no longer predictive and neurology was most strongly associated (neurology>surgery>internal medicine; $p=0.007$ ) and for non-probabilistic statements, patient age was no longer predictive.

\section{Conclusions}

Prognostication occurred frequently but varied greatly by language used, outcome discussed, clinician and patient characteristics. Next, we will examine whether the type of prognostic language predicts prognostic misunderstanding by families. 


\section{Poster 132}

\section{CXCL14 Gene Methylation and Delirium in Critically ill Patients.}

Haydar Al Ebousi, Yvette Conley, Dianxu Ren, Sheila A. Alexander

University of Pittsburgh/ School of Nursing, Pittsburgh, PA, United States

\section{Background \& Purpose}

Delirium is an acute cognitive impairment which occurs commonly in the intensive care units (ICU). Delirium is associated with a longer ICU and hospital stay, longer mechanical ventilation, increased resource utilization, higher mortality and cognitive dysfunction after discharge. Patients with delirium have higher serum pro-inflammatory cytokines, however the individual factors driving this phenomenon have not been identified. DNA methylation decreases gene expression. CXCL14 protein is chemotactic for monocytes. In this pilot study, we examined temporal variability in CXCL14 gene methylation in ICU subjects who do/do not develop delirium.

\section{Methods}

This study included 10 adults admitted to a Medical or Surgical-Trauma ICU, mechanically ventilated for at least 24 hours and without history of cognitive dysfunction. Delirium was assessed with the Confusion Assessment Method ICU and blood samples were drawn from existing catheters daily for five days. DNA was extracted and methylation quantified with the EpiTect Methyl II PCR Array Human Inflammatory Response and Autoimmunity Signature Panel (Qiagen Inc.). We performed descriptive analysis and t-test to determine differences in CXCL14 methylation on days 1,3,5.

\section{Results}

Subjects were primarily male (60\%), non-Hispanic (100\%), and White $(100 \%)$ with a mean age of 58.70 years old. 70\% developed delirium. Day 1 CXCL14 methylation was lower in subjects who developed delirium (0.62\%) compared to those without delirium (1.35\%; $t=2.75, p=.025)$.

\section{Conclusions}

Our findings suggest that CXCL14 methylation could be an individual contributor to the development of ICU, although the sample size and effect size are very small for this pilot study. Studies with larger samples and exploring more inflammatory genes are needed to further define the source of individual variability in delirium development. 
Poster 133

\title{
Effects of Positive End Expiratory Pressure on Intracranial Pressure and Cerebral Perfusion: A Systematic Review
}

\author{
Sharoon Samuel, Muhammad Ans Sharif, Brent Brown, Sechme Khan, Paul Berger III \\ University of South Dakota, Department of Medicine, Sioux Falls, SD, United States
}

\section{Background \& Purpose}

Hypoxic patients on ventilatory support and positive end expiratory pressure (PEEP) have evidence of reduced cerebral venous return resulting in increased intracranial pressure (ICP). This systematic review studies the clinical significance of PEEP on ICP and cerebral perfusion.

\section{Methods}

We performed a search for randomized controlled studies on the effect of PEEP on ICP and cerebral perfusion using the following databases: Pub med, Embase, AdisInsight, and Cochrane. Studies involved in effect of PEEP on ICP irrespective of age, sex, or specific eligibility criteria were included.

\section{Results}

Out of 3260 we included 11 studies with 509 patients. A 2002 study by Videtta et al on traumatic brain injury $(n=10)$ had patients with PEEP of 5, 10 and 15 ; there was an increase in intracranial pressure with increasing PEEP $p<0.049$ with no change in cerebral perfusion pressure $(p=0.819)$. Boone et al in 2017 studied ICP and PEEP in 341 patients. Four cohorts were made based on severity of lung injury. A relationship between PEEP and ICP persisted only in severe lung injury group. For every $\mathrm{cmH} 2 \mathrm{O}$ increase in PEEP, there was an increase in ICP of $0.31 \mathrm{mmHg}(95 \% \mathrm{Cl}[0.07,0.54])(P=0.04)$. Another study with 9 hypoxemic patients on PEEPmax of $21 \mathrm{mmHg}$ showed no statistically significant change in ICP on PEEPmax as compared to zero PEEP. A 1985 study with PEEPmax of 10 showed an increase in ICP of $1.3 \mathrm{mmg}$ in 17 patients $(\mathrm{N}=33)$. Nemer et al in their study showed high PEEP in patients is safe to improve brain oxygenation without concern of clinically significant increase in ICP.

\section{Conclusions}

Higher PEEP level statistically increases ICP to a minimal degree in majority of studies. Further studies need to be done to assess clinical significance of the relationship between ICP \& PEEP. 


\section{Poster 134}

\section{End-of-Life Care in the Potential Donor after Circulatory Death: A Review}

Stefanie P. Cappucci, Wade S. Smith Richard Schwartzstein, Douglas B. White, Susan L. Mitchell, Corey R. Fehnel

Beth Israel Deaconess Medical Center, Neurology Department, Boston, MA, United States

\section{Background \& Purpose}

Donation after circulatory death (DCD) is becoming increasingly common, and yet little is known about the way potential donors receive end-of-life care. Potential DCD patients are a heterogenous population of the neuro-critically ill. The primary objective was to describe palliative and comfort focused care for DCD patients at the end-of-life and examine metrics currently used to assess discomfort among these patients.

\section{Methods}

Systematic review of published literature between June 1, 2000 and June 31, 2020 of end-of-life care received by potential DCD patients. The population of interest was defined as patients eligible for Maastracht classification III donation after circulatory death for a solid organ transplantation. Outcomes examined included: analgesic or palliative protocols, and surrogates of discomfort (e.g. pain, dyspnea, agitation).

\section{Results}

Among 141 unique articles, 27 studies were included for full review. The primary reason for exclusion of identified studies was lack of protocol description, or of reporting on use of analgesic or sedative medications. No primary research studies specifically examined distress in the DCD eligible population. Numerous institutional/professional guidelines, which were based on expert opinion, were identified. Surveys of critical care practitioners identified concerns regarding the impact of symptom management on hastening the dying process in the DCD population as a potential barrier to end-of-life palliative treatment.

\section{Conclusions}

There is a paucity of empirical evidence for end-of-life symptom assessment and management for DCD patients. Neurocritical care practioners have a distinctive role in the care of patients being considered for organ donation. In order to support the development of interdisciplinary guidelines, key evidence gaps identified include: i) defining a multidisciplinary structure of treatment teams and preferred environment for DCD, ii) objective tools for monitoring of distress, and iii) evidence guiding the administration of sedative and analgesic medications following withdrawal of life sustaining therapy among the DCD population. 


\title{
Poster 135
}

\section{Evaluation of the 2015 AAN Inpatient and Emergency Quality Measures}

\author{
Juliana Bartel, Katherine Kowalski, Juliana Bartel, Sarah Livesay Yvonne Tumbali, Andrew Dorsch
}

Rush University, Chicago, IL, United States

\section{Background \& Purpose}

The American Academy of Neurology (AAN) published a quality measure set to promote quality of care and improve outcomes among critically ill inpatient and emergency neurology patients (Josephson et al., 2017). We conducted a single center evaluation at a large academic urban medical center to identify availability of the data required for the collection of these quality measures, generate initial metric outcomes, and formulate recommendations for future collection.

\section{Methods}

The evaluation was guided by the Donabedian framework and included an electronic health record (EHR) feasibility assessment and chart review for each measure. The outcome analysis guided discussions about sustainability of continued measure collection.

\section{Results}

This project evaluated 9 of the 12 AAN quality measures. Current medical coding and EHR documentation posed significant challenges in 6 of 9 measures. Documentation consistent with reliable metric measurement was found in 5 of the 9 measures (brain death documentation, bacterial meningitis, myasthenic crisis (MG), status epilepticus (SE) treatment, and Guillain Barre Syndrome (GBS). Several measures were limited by a small sample size. Measures that relied on the medication administration record (MAR) were reliably extracted. Coding and documentation posed a significant challenge for the $\mathrm{SE}, \mathrm{EEG}$, goals of care and advanced directive measures. Measure compliance was noted to be $>90 \%$ in 6 of the 9 measures, with poor compliance noted in the bacterial meningitis, goals of care and advanced directive measures. All measure extraction required a manual workflow.

\section{Conclusions}

Current documentation and medical coding pose a challenge when implementing the AAN inpatient quality measure set. Reliable extraction of the measures requires standardized documentation in the EHR. The current measure language for SE measures and definition of coma were barriers to measure extraction. 


\section{Poster 136}

\section{Exploring Staff Understanding of Code Status and Treatment Limitations at UW Health}

Derek Steck, Michael Pulia, Joshua Medow, Zhanhai Li, Margaret Schwarze, Manish Shah, Marin Darsie

University of Wisconsin School of Medicine, Madison, WI, United States

\section{Background \& Purpose}

Given the high-stakes nature of care during cardiopulmonary arrest, the appropriate course of action should be clear and based on a patient's stated code status. UW Health's code status options recently transitioned from "Full Code," "Limited," and "DNR (do not resuscitate)" to "Full Code," "No CPR/May intubate for potentially reversible conditions," and "No CPR/Do not intubate for any reason." "No CPR" was intended to be synonymous with "DNR." We aimed to determine the prevalence of confusion surrounding code status definitions among UW health clinical providers and how this translated to patient management in clinical vignettes.

\section{Methods}

After conducting semi-structured interviews of nine employees, a 17-question survey was developed to explore the interpretation and application of UW Health code status options in hypothetical cardiac arrest and non-arrest clinical scenarios. The survey was distributed to all UW Health providers and nurses working in the emergency department, surgical and medical wards, and ICUs. We hypothesized that at least one-third of respondents would indicate a willingness to utilize chest compressions, defibrillation, and/or intravenous medications for some cardiac arrest scenarios despite a "No CPR" code status, which would be inappropriate per UW Health's No CPR Clinical Policy \#1.2.13.

\section{Results}

The survey response rate was $21 \%(239 / 1,137)$. In total, 120 nurses, 24 advanced practice providers, and 95 physicians responded. $72.8 \%$ of respondents indicated that they would use chest compressions, defibrillation, and/or intravenous medications to treat cardiac arrest in some situations despite a "No CPR" code status. Finally, respondents were asked to read Clinical Policy \#1.2.13; 32.6\% of respondents indicated that they would change at least one of their survey answers after reading it.

\section{Conclusions}

There is significant confusion surrounding the "No CPR" code status among UW Health nurses and providers. Our findings suggest the need for better education on the No CPR Clinical Policy \#1.2.13 and its clinical application. 


\section{Poster 137}

\section{High-Flow Nasal Cannula Fails to Reduce Re-intubation Rates in Neurologic Intensive Care Unit}

Tijo Thomas, Jamie Bloom, Victor Romo, Marc Torjman, Muhammad Athar, David Wyler, Jacqueline Urtecho

Thomas Jefferson University Hospital, Philadelphia, PA, United States

\section{Background \& Purpose}

High-flow nasal cannula (HFNC) can decrease re-intubation rates in respiratory failure. Neurologically injured patients are considered high-risk of re-intubation due to bulbar dysfunction and neurological deficits. We examined outcomes of neurologically injured patients with post-extubation respiratory failure with use of HFNC compared to standard oxygen therapy (SOT).

\section{Methods}

Retrospective analysis of neurologically injured patients admitted from 4/2017-1/2020 requiring mechanical ventilation and extubated to HFNC versus SOT. Primary outcome was re-intubation rate. Secondary outcomes were rates of aspiration and VAP.

\section{Results}

There was no significant difference between HFNC and SOT groups APACHE II (mean 14.7 vs 15.9,p0.31). There was no significant difference in the incidence of comorbid diseases. Stroke was more common in the HFNC group ( $33.3 \%$ vs $15.9 \%$ SOT, $p<0.044$ ); other primary diagnoses were similar in both groups. HFNC group had more ventilator days (5.1 vs 3.7 days, $P=0.027$ ). Prior to extubation, gag reflex was present less frequently with $\operatorname{HFNC}(58.3 \%$ vs $88.4 \%, p=.001)$ while presence of cough and copious secretions were similar in both groups. Incidence of post-extubation pneumonia did not differ between groups. There was no difference in length of ICU stay, ICU readmission, hospital length of hospital stay, or survival at discharge. The reintubation rates were similar in both groups. However, the HFNC group had higher rates of aspiration events $(41.7 \%$ vs $17.9 \%, p=0.047)$ and need for tracheostomy (62.5\% vs $26.7 \%, p=0.013)$.

\section{Conclusions}

For patients requiring intubation following neurologic injury, HFNC was not associated with decreased rates of re-intubation. A higher incidence of stroke, longer time to extubation, and increased incidence of absent gag reflex in the HFNC group may have contributed to the increased incidence of aspiration events and need for tracheostomy in this group. Further evaluation of HFNC in NeurolCU patients is required. 
Poster 138

Impact of Coronavirus (COVID-19) on Partial Pressure of Carbon Dioxide (CO2) Reactivity

Jamie Nicole LaBuzetta, Valerie Arias, Arthur Lam, Mazen Odish, Robert Owens

University of California San Diego - Neurocritical Care Department, San Diego, CA, United States

\section{Background \& Purpose}

Studies have demonstrated that patients with coronavirus disease (COVID-19) have an increased incidence of acute cerebrovascular complications. We theorize that increased cerebrovascular risk is related to abnormal cerebral blood flow (CBF) dynamics, and therefore measured CBF characteristics in the middle cerebral artery (MCA) using transcranial Doppler ultrasound (TCD).

\section{Methods}

In this prospective, IRB-approved study, ventilated patients with acute respiratory distress syndrome secondary to COVID-19 without historical or acute neurological diagnosis were screened for inclusion. The FDA-approved Lucid Robotic system ${ }^{\mathrm{TM}}$ recorded baseline velocity in bilateral MCAs (VMCA) and pulsatility index $(\mathrm{PI})$. To evaluate $\mathrm{CO} 2$ reactivity, the respiratory rate was adjusted to maintain a $\mathrm{PaCO} 2$ of 2-5 $\mathrm{mmHg}$ below each patient's baseline for five minutes; TCD measurements were then repeated. To assess vasomotor reactivity, a paired t-test was used to compare pre- and post-intervention values with significance set at $p<0.05$.

\section{Results}

Six patients (Male: 67\%) were included. Mean age was 55 years. Mean P/F ratio was 180.5 . On average, patients were studied 9.5 days (range 1-23) post-intubation, 22.5 days (range 7-51) after symptom onset, and 17.5 days (range 3-45) after their first positive PCR test. Baseline mean Right-VMCA was 58.5 $\mathrm{cm} / \mathrm{s}$ and Left-VMCA was $54.4 \mathrm{~cm} / \mathrm{s}$. No significant differences were noted in VMCA following hyperventilation (Right-VMCA change: $+3.0,95 \% \mathrm{Cl}-1.03$ to 5.69 ; Left-VMCA: $+2.3,95 \% \mathrm{Cl}-0.83$ to 6.83 ). Additionally, no significant difference was identified in the Right-PI (Mean baseline: $0.77 ; 95 \% \mathrm{Clchange:} \mathrm{-}$ 0.05 to 0.06 ) or Left-PI (Mean baseline: $0.97 ; 95 \%$ Clchange: -0.12 to 0.08 ). One patient developed a lacunar stroke within one month of data collection.

\section{Conclusions}

$\mathrm{CO} 2$ reactivity is thought to reflect cerebrovascular reserve. While limited by a small sample size, the lack of significant change in CBF following changes in $\mathrm{PaCO} 2$ suggests that impaired cerebrovascular reactivity may contribute to increased risk of neurological disease in COVID-19. 


\title{
Poster 139
}

\section{Insurance Reimbursement for Artificial Intelligence and New Technology in Acute Stroke and in the Neurocritical Unit}

\author{
Nick Murray, Ferdinand Hui
}

Stanford University, Division of Neurocritical Care, Palo Alto, CA, United States

\section{Background \& Purpose}

Insurance reimbursement in stroke and Neurocritical Care is rapidly evolving to accommodate novel technologies. In late 2020, the Centers for Medicare and Medicaid Services (CMS) approved of the first ever New Technology Add-on Payment (NTAP) that reimbursed for artificial intelligence (AI) technology use for triage of large vessel acute ischemic stroke. Here, we analyze the science and economic strategies used for reimbursement approval and characterize how the NTAP holds unique value for AI used in Neurocritical Care.

\section{Methods}

Literature and United States government document analysis.

\section{Results}

There is a mismatch between the (1) cost for using novel Al software in stroke triage and acute neurocritical care and (2) the subsequent reimbursement from insurance payers. With use of new Al technology in routine patient care, reimbursement will not yet exist from the insurance payer, often lagging from time of Al introduction by one to three years, or until it's cost and effect on care is validated. The NTAP addresses this mismatch by offering timely reimbursement. We analyze the economic science, current state of NTAP, and specific components of the NTAP relevant for neurointensivisits that may use or are using Al. By in-depth analysis of the first use case in acute stroke triage, multiple strategies are required to gain reimbursement. In the first approved case, the Al demonstrated a unique mechanism of action, notification via a form of robotic process automation, and linking of efficiency afforded to clinical outcomes. Other forms of Al are likely to see qualification for NTAP reimbursement by meeting three criteria: fiscally quantifiable, explainable output, and clinically actionable.

\section{Conclusions}

The NTAP supports the accelerating rate of change in healthcare technology using Al by mitigating the risks of adoption by providing reimbursement for such technology. There are multiple strategies based on economic and clinical science that enable specific Al to take advantage of the NTAP. 
Poster 140

\section{Less is More: Reducing ICU Vampirism in a Neurocritical Care Unit}

Melissa Rentz, Susan Yeager, Archana Hinduja

Ohio State University Wexner Medical Center, Neurocritical Care, Columbus, OH, United States

\section{Background \& Purpose}

Critical care units are challenged to reevaluate historical diagnostic testing practices. This includes the daily ordering of laboratory tests. While necessary to provide care for critically ill patients, room for improvement exists to minimize waste and healthcare costs. The purpose of this poster is to outline a Neurocritical care unit (NCCU) initiative to reduce laboratory utilization and costs. The NCCU in a Midwestern, academic, comprehensive stroke, medical center traditionally orders at minimum daily screening labs on all patients. A brief review of the literature found several critical care quality improvement initiatives to safely decrease laboratory utilization, and healthcare costs. A few of these interventions included staff educational sessions, monthly progress feedback, audits, and posted reminders.

\section{Methods}

A single-center retrospective and prospective cohort study was conducted to compare pre and post patient groups in terms of number of laboratory tests ordered and costs associated with these tests. Adult patients in the NCCU were included in this study. A team composed of physicians, nurse practitioners, and nursing staff was established and protocols developed. Data collected included the number and types of laboratory tests ordered. The sample size was determined by the number of patients who were treated in the NCCU from December 2018 to March 2019 compared to the same period in 2019-2020.

\section{Results}

A total of $33.2 \%$ reduction was noted from pre to post intervention. Evaluating only CBC with electronic differential, a total of 206,080 labs were drawn baseline with 95,520 post intervention. This reduction resulted in a total cost savings of $\$ 110,560$. Chem 7 had 2359 baseline draws with 2274 post intervention. The 85 lab difference resulted in a $\$ 27,963$ cost savings.

\section{Conclusions}

It is possible to implement lab reduction strategies in the NCCU resulting in a total lab decrease and associated cost savings. 


\title{
Poster 141
}

\section{Leveraging the Capabilities of Al: Neurology Trained Providers with Novice Experience Performing Cardiac POCUS in Patients with Acute Brain Injury}

\author{
Jennifer Mears, Rohan Panchamia, Prajwal Ciryam, Cenai Zhang, Richard Thalappillil, Christopher W. \\ Tam, Judy H. Ch'ang
}

New York Presbyterian Weill Cornell Neurocritical Care, New York, NY, United States

\section{Background \& Purpose}

Cardiac point-of-care ultrasound (CPOCUS) aids in diagnosis and treatment of cardiac complications from acute brain injury. Many neurology providers have not been trained in ultrasound. Caption Al uses a novel deep-learning (DL) algorithm to guide novice POCUS users in obtaining diagnostic cardiac images for interpretation. We hypothesized that with this DL software, neurology providers with minimal POCUS experience would capture quality images and that these studies would change management or decrease time to screening echoes in the neurological ICU.

\section{Methods}

Over seven months, neurology trained physician assistants and fellows used DL software to perform cPOCUS studies. Questionnaires assessed the purpose for cPOCUS: screening, education or acute, and if it changed management. Certified echocardiographers evaluated each scan independently to assess interpretability of LV function, RV function, IVC size, and presence of a pericardial effusion. Descriptive statistics with exact confidence intervals were used to report crude rates. Time to obtain initial screening CPOCUS was compared to a similar population of first screening TTEs from 2018. The difference was calculated using the Wilcoxon rank-sum test. Interrater reliability was calculated using Krippendorff's alpha.

\section{Results}

Of 53 patients, 68 studies were performed with a total of 312 images. Three over-readers deemed $88.2 \%$ (95\% Cl, 86.0\%-90.1\%) of these images as interpretable for a qualitative assessment of LV size and function, $66.2 \%$ (95\% Cl, 63.2\%-69.1\%) for RV size and function, 56.4\% (95\% Cl, 53.3\%-60.0\%) for IVC size and variability, and $74.5 \%(95 \% \mathrm{Cl}, 71.7 \%-77.2 \%)$ for the presence of a pericardial effusion. Interrater reliability was Kalpha $=0.736 .44 .6 \%$ of all studies changed management. Most common management changes were adjusting fluid goals and vasopressor use. Time to obtain screening cPOCUS decreased by 1 day $(p=0.002)$.

\section{Conclusions}

With DL guidance, neurology providers with minimal to no POCUS training were able to obtain diagnostic quality cardiac images which led to acute management changes and significantly decreased time to screening echo. 


\section{Poster 142}

\section{Machine Learning for Intensive Care Delirium Prediction}

Kirby Gong, Ryan Lu, Teya Bergamaschi, Akaash Sanyal, Joanna Guo, Han B. Kim, Robert D. Stevens.

Whiting School of Engineering, Johns Hopkins University, Baltimore, MD, United States

\section{Background \& Purpose}

ICU delirium is frequent, associated with unfavorable outcomes, increased costs, and may be preventable. There is an unmet need for accurate methods to predict risk of delirium. Here, we trained machine learning models to predict ICU delirium using physiological and clinical features in two large clinical databases.

\section{Methods}

Data were obtained from the multi-center eICU database. Only features from the first $24 \mathrm{~h}$ following ICU admission were extracted. The first model $(A)$ was designed to predict delirium onset at any point during the ICU stay. A second "dynamic" model (B) was built to predict onset of delirium in an hour. For both models, three algorithms were evaluated: logistic regression [LR], random forest [RF], and gradient boosting [CatBoost]). Model performance was evaluated using nested cross-validation and area under the receiver operating characteristic curve (AUROC), precision-recall, and calibration metrics, and compared to the established PRE-DELIRIC model. Top features were analyzed using LR, RF, or Shapley values. Similar features were extracted from the MIMIC-III database for external validation.

\section{Results}

Among 18,302 patients admitted to the ICU, 2,536 (16\%) had delirium. For model $A$, the highest mean AUROC was 0.791 , significantly better than PRE-DELIRIC ( $p=.003)$. Model B had a mean AUROC of 0.889 . Among the top features in both models were GCS, RASS, APACHE scores, and age. Performance of both models was comparable when externally validated in MIMIC-III. Physiological time series features did not increase either model's performance.

\section{Conclusions}

Machine learning algorithms trained with data from the first $24 \mathrm{~h}$ after admission predicted ICU delirium with greater accuracy than the reference PRE-DELIRIC score. Results suggest that high-resolution data contain predictive information on delirium risk overlooked in current prediction systems. A dynamic model trained with data from hours before the onset of delirium had even greater predictive performance. These results warrant additional validation and enrichment with other features such as neurophysiological time series and neuroimaging. 


\section{Poster 143}

\section{Neurocritical Care Performance Measures Derived from Electronic Health Record Data are Feasible and Reveal Site-Specific Variation: Results from the CHoRUS Workgroup}

Sophie E. Ack, Shamelia Y. Loiseau, Guneeti Sharma, Joshua N. Goldstein, PhD, India A. Lissak, Sarah M. Dougherty, Neha Dangayach, Edilberto Amorim, Paul Vespa, J. Randall Moorman, Xiao Hu, Gilles Clermont, Brandon P. Foreman, Eric S. Rosenthal

Massachusetts General Hospital, Department of Neurology, Boston, MA, United States

\section{Background \& Purpose}

We evaluated the feasibility and discriminability of previously described neurocritical care quality indicators and performance measures (Livesay, Neurocritical Care, 2019; Huijben, Critical Care, 2020) extracted from electronic health record (EHR) flowsheet data from three centers within the Collaborative Hospital Repository to Unlock Synergy (CHoRUS) workgroup.

\section{Methods}

We included consecutive neurocritical care admissions exceeding 24 hours at three institutions (03/2015-02/2020), and evaluated the feasibility and discriminability of five clinical performance measures: 1) ICP monitoring (ICPM) when indicated, 2) ICPM latency when occurring within 24 hours, 3) frequency of nurse-documented neurologic assessments, 4) intermittent pneumatic compression device (IPCd) application within 24 hours, and 5) latency to IPCd application. We additionally explored associations between delayed IPCd application and ICD-10-documented in-hospital venous thromboembolism (VTE). Median [IQR], Kruskal-Wallis, and Dunn statistics are reported.

\section{Results}

14,985 admissions met inclusion criteria. ICPM, neurologic assessments, and IPCd application were documented for $1,514(10.1 \%), 14,635$ (91.1\%), and 14,175 (88.5\%) admissions, respectively. ICPM began within 24 hours for 1,267 (83.7\%) with site-specific latency differences among sites 1-3, respectively, $(0.54 \mathrm{~h}[2.82], 0.58 \mathrm{~h}[1.68]$, and $2.36 \mathrm{~h} \mathrm{[4.60];} \mathrm{p}<0.001)$. The frequency of nursedocumented neurologic assessments varied across sites (17.4/day [5.97], 8.4/day [3.12], and 15.3/day [8.34]; $p<0.001$ ). IPCd application occurred within 24 hours for 12,863 (90.7\%) of eligible patients, excluding those who refused prophylaxis, had limiting injuries, or were actively documented as ambulating. In-hospital VTE at each site was associated with increased IPCd latency (2.58h [10.27] vs. $1.92 h$ [7.75], $p=0.134 ; 1.45 h$ [7.54] vs. $0.78 h$ [3.40], $p=0.006 ; 0.80 h$ [1.40] vs. $0.68 h$ [3.82], p=0.087). VTE diagnosis also varied by site $(1.23 \%, 1.55 \%$, and $5.18 \%$; $p<0.001)$.

\section{Conclusions}

EHR-derived reporting of neurocritical care performance measures is feasible and demonstrates sitespecific variation. Future efforts should examine multivariate associations between performance measures and outcomes. 


\title{
Poster 144
}

\section{Ordering and Performing Provider Attitudes Towards Hourly Neuroassessments}

\author{
Max Kazer, Lauren Stone, Biren Kamdar, Atul Malhotra, John Evans, Robert Owens, Jamie Nicole
} LaBuzetta

University of California, San Diego Department of Neurosciences, San Diego, CA, United States

\section{Background \& Purpose}

Hourly neuroassessments (HNA) are used to serially monitor and rapidly identify neurological deterioration in patients with acute brain injury ( $A B I)$, but may unintentionally hinder recovery. We investigated intensive care unit (ICU) healthcare providers' attitudes towards HNA at an academic medical center.

\section{Methods}

Providers from multiple ICU services including attendings, trainees, and advanced practice providers ("ordering providers") and bedside nurses ("performing providers") were invited to complete an IRBapproved survey reviewed by a methodologist consisting of multiple choice, Likert scale, and write-in questions. Participant informed consent was obtained.

\section{Results}

172 surveys were completed (36\% "ordering providers"; $69 \%$ with neurosciences specialty). Providers reported ordering HNA for $76 \%$ of patients with ABI. "Standard of care" (85\%), "unstable clinical status" (85\%), and "specific diagnoses" (78\%) were the most frequent reasons for ordering HNA, but the providers' decisions were primarily influenced by "standard of care" (75\%). Providers were least willing to discontinue HNA for non-English speaking and/or aphasic patients. Providers estimated ordering HNA for 2.6 days on average. $54 \%$ of providers believed patients with $A B I$ should receive neuroassessments every 2 hours, with only $17 \%$ of providers reporting they would want HNA for themselves following ABI. Nurses reported performing HNA as ordered only $63 \%$ of the time. The most common reasons for incomplete HNA included patient agitation, language barrier, and patient non-compliance, with patient agitation impacting nurse willingness to perform HNA the most. $69 \%$ of nurses believed patients with $A B I$ should receive neuroassessments every 2 hours, with $22 \%$ wanting HNA for themselves following $\mathrm{ABI}$.

\section{Conclusions}

HNA are common following ABI with "standard of care" playing a large role in the decision to order them. However, the majority of ordering and performing providers believe HNA to be too frequent following $A B I$ and would not want HNA for themselves following $A B I$. 


\section{Poster 145}

\section{Paroxysmal Sympathetic Hyperactivity After Non-Traumatic Acute Brain Injury: Preliminary Data}

Yong-Bum Song, Hemal Patel, Siddhart Mehta, ChHassan Ali

JFK University Medical Center - Neuroscience Institute, Edison, NJ, United States

\section{Background \& Purpose}

Current literature suggests that paroxysmal sympathetic hyperactivity (PSH) is most commonly associated with traumatic brain injury. Few isolated cases of PSH after ischemic stroke, hydrocephalus, autoimmune encephalitis, and cerebral fat embolism have been reported. The prevalence of PSH secondary to non-traumatic brain injury is still not clearly understood.

\section{Methods}

We performed prospective data collection of consecutive patients admitted to non-trauma center academic university hospital with a specialized Neurocritical Care Unit (NCCU) during 3-month period (January - March 2021) to identify patients with possible PSH. We identified PSH with the presence of at least three of the following six clinical features: fever, tachycardia, hypertension, tachypnea, sweating, and posturing. Infectious, inflammatory, and/or toxic-metabolic etiologies were ruled out.

\section{Results}

Possible PSH was identified in eight patients (male $=6$, age $=51$ [27 - 85]). 4/8 patients had intracranial hemorrhage $(\mathrm{ICH}): 2$ with basal ganglia, 1 with cerebellar, and 1 with fronto/temporal ICH. 4/8 patients had acute ischemic stroke: 3 with cerebellar, and 1 with MCA. 2 had all six clinical features of PSH (Temp $=\left[101.4-105^{\circ} \mathrm{F}\right], \mathrm{HR}=[125-135], \mathrm{SBP}=[128-190], \mathrm{RR}=[28-38],+$ sweating, and + posturing) 1 with five (Temp $=101.1^{\circ} \mathrm{F}, \mathrm{HR}=110, \mathrm{SBP}=168, \mathrm{RR}=27$, and + sweating), 2 with four (Temp $=[100.4-$ $\left.104{ }^{\circ} \mathrm{F}\right], \mathrm{HR}=[102-132], \mathrm{SBP}=[156-186]$, and $\left.\mathrm{RR}=[20-39]\right)$, and 3 with three $($ Temp $=[98.6-102.2$ $\left.{ }^{\circ} \mathrm{F}\right], \mathrm{HR}=[88-130], \mathrm{SBP}=[167-174]$, and $\left.\mathrm{RR}=[15-29]\right)$.

\section{Conclusions}

Our data indicates that PSH is commonly encountered among our non-traumatic acute brain injury patients in NCCU. Prompt identification of PSH in this patient population would be crucial in guiding appropriate pharmacotherapy management. Prospective cohort study is warranted to evaluate further prevalence in this patient population. 


\title{
Poster 146
}

\section{Persistent Viral RNA Shedding is Associated with Delirium Incidence and 6-Month Mortality in COVID- 19 Patients Requiring Hospitalization}

\author{
Anthony K. Kang, Sareen Ali, Tulsi R. Patel, Jeffrey R. Clark, Nathan A. Shlobin, Steven C. Hoffman, \\ Zachary S. Orban, Patrick H. Lim, Edith L. Graham, Ayush Batra, Eric M. Liotta, Igor J. Koralnik
}

Northwestern University Feinberg School of Medicine, Ken \& Ruth Davee Department of Neurology, Chicago, IL, United States

\section{Background \& Purpose}

Increasing reports of persistently positive SARS-CoV-2 tests in coronavirus disease 2019 (COVID-19) patients and the associated public health implications have raised interest in the mechanisms surrounding SARS-CoV-2 material shedding. We sought to investigate the implications and outcomes associated with persistent SARS-CoV-2 viral shedding in patients requiring hospitalization for COVID-19.

\section{Methods}

We retrospectively examined the presence of persistent viral material shedding within a cohort of patients hospitalized for COVID-19 in a Chicago area hospital network between March and August 2020. Persistent shedding was defined by positive SARS-CoV-2 RT-PCR assay of nasopharyngeal or bronchoalveolar lavage samples at least 14 days after initial positive RT-PCR assay. We assessed for predictors of persistent viral shedding, in-hospital delirium, and 6-month mortality using binary logistic regression.

\section{Results}

Of 2,518 admitted patients, 959 (38.1\%) underwent repeat SARS-CoV-2 RT-PCR testing at least 14 days after initial positive testing, in whom persistent viral shedding was identified in 405 (42.2\%) patients. In unadjusted analysis, persistent shedding was associated with male sex, increased BMI, diagnoses of diabetes mellitus and chronic kidney disease, and corticosteroid exposure during COVID-19 hospitalization. Persistent shedding was associated with Confusion Assessment Method-defined delirium during hospitalization in unadjusted analysis and remained independently associated with delirium (adjusted OR 2.45 [95\% $\mathrm{Cl} 1.75,3.45]$ ) after adjusting for factors including respiratory dysfunction severity. Persistent shedding remained significantly associated with increased 6-month mortality (adjusted OR 2.43 [95\% Cl 1.42, 4.29]) even after adjusting for age, respiratory dysfunction severity, and occurrence of delirium.

\section{Conclusions}

Persistent viral shedding following SARS-CoV-2 infection in hospitalized COVID-19 patients is associated with increased mortality at six months. The association between in-hospital delirium and persistent viral material shedding suggests a potential underlying morbid mechanism with neurologic implications that is present during the acute phase of illness. 
Poster 147

\section{Pharmacogenomics (PGx) Associations of NeurolCU Anti-Seizure Drugs (ASD) and Life-Threatening Skin Reactions}

Krina D Vyas, Toni Turnbull, Sarah Peacock, Ashley Rogers, Devang Sanghavi, Daniel Jackson, W. David Freeman

Mayo Clinic, Pharmacy, Jacksonville, FL, United States

\section{Background \& Purpose}

Pharmacogenomics (PGx) is an emerging area of clinical research with increasing amounts of practical implications for various patient populations. The goal of utilizing PGx in clinical practice is to predict variability in drug response between individuals. This is particularly important in critically ill patients as physiologic alterations already affect the pharmacokinetic and pharmacodynamic properties of drugs. In this review, we explore the role of PGx in precision medicine to discuss common and serious adverse drug reactions (ADRs) and greater recognition of potential life-threatening anti-seizure drugs (ASD) used in the neurointensive care unit (neuroICU) for status epilepticus.

\section{Methods}

We reviewed pertinent literature to illustrate important concepts of PGx using the National Library of Medicine of anti-seizure drugs with ADR's of Stevens Johnson syndrome (SJS), Toxic Epidermal Necrolysis (TEN), and Drug Rash with Eosinophilia and Systemic Symptoms (DRESS) and ethnic backgrounds at risk.

\section{Results}

Up to $95 \%$ of hypersensitivity reactions have been reported in regard to aromatic antis-seizure drugs. These directly interact with human leukocyte antigen (HLA) to develop a hypersensitivity response. Additionally, drug reaction with eosinophilia and systemic symptoms are often unrecognized in the neurolCU as patients are intubated. Furthermore, cross-reactivity or drug-drug interactions among ASDs are reported to be up to $80 \%$. PGx literature demonstrates that certain ethnic backgrounds are at higher risk for life-threatening skin reactions.

\section{Conclusions}

There are known ethnic PGx diversity genotypes at higher risk for serious ADR including SIS/TEN and DRESS. Awareness of certain PGx ethnic populations for serious anti-seizure drug skin reactions like SJS could lead to earlier intervention and change to newer ASDs without these risks and might potentially avoid life-threatening skin reactions. 


\title{
Poster 148
}

\section{Pharyngeal Electrical Stimulation for Promoting Recovery from Dysphagia after Brain Injury or Prolonged Ventilation: A Scoping Review}

\author{
Stephan A. Mayer, Theresa Henson, Cathra Halabi, Wade S. Smith, Neeraj Badjatia \\ New York Medical College, Valhalla, NY, United States
}

\section{Background \& Purpose}

Neurogenic dysphagia (ND) occurs frequently after brain injury or prolonged mechanical ventilation (MV). Pharyngeal electrical stimulation (PES) drives neuroplastic reorganization and is a novel treatment for ND. PES is delivered via a nasogastric tube that provides $5 \mathrm{~Hz}$ electrical current to the oropharynx. Treatment is given for 10-minutes/day for 3-6 days.

\section{Methods}

We searched electronic databases for clinical research studies investigating transnasal PES for treatment of ND after stroke, traumatic brain injury (TBI) or prolonged MV. Data abstraction was performed and verified by two reviewers. Quantitative and qualitative syntheses were conducted.

\section{Results}

Ten studies reporting 662 patients were identified. The most common use scenarios were nonventilated acute stroke ( $<14$ days) ( $\mathrm{N}=349$ ); stroke or TBI with tracheostomy after weaning from MV $(\mathrm{N}=244)$; non-stroke or TBI patients treated with $\mathrm{MV}(\mathrm{N}=35)$; and chronic stroke ( $>6$ months, $\mathrm{N}=6)$. Six clinical trials randomized a total of 341 stroke patients to PES or sham therapy. After 3 small positive trials, the largest trial (STEPS, $\mathrm{N}=162$ ) failed to demonstrate improved penetration-aspiration scores after PES, which was attributed to low levels of electrical stimulation and exclusion of patients with severe dysphagia. Two subsequent trials (including PHAST-TRAC) in tracheostomized stroke patients after weaning $(\mathrm{N}=99)$ reported a dramatic increase in the proportion of patients who could be decannulated within 24-72 hours after PES (58\% vs $11 \%$ ). In PHADER, an uncontrolled single-arm registry ( $N=239)$, PES led to improved measures of dysphagia and aspiration in all clinical subgroups, including after longstanding dysphagia. A sub-study of 15 patients treated with PES during orotracheal intubation in the PHADER cohort showed a lower late of reintubation than historical controls.

\section{Conclusions}

PES is a promising treatment for ND. Further trials are needed to establish clinical use scenarios in the ICU and stroke unit that improve outcome, length of stay, and quality of life. 


\section{Poster 149}

\section{Post neuRo intEnsiVe cAre vIrtual cLinic (PREVAIL)}

Sarah Street, Lindsay Bruening, Christine Ahrens, Jessica Biedny, Sarah Beinkampen, Kathy Leestma, Nicholas Kwolek, Joao A. Gomes

Cleveland Clinic Neuro Intensive Care Unit, Cleveland, $\mathrm{OH}$, United States

\section{Background \& Purpose}

Post ICU clinics, primarily focused on medical and surgical intensive care patients, have worked to address the constellation of symptoms seen following critical illness. We launched the Post neuRo intEnsiVe cAre virtual cLinic (PREVAIL) to address issues unique to patients with acute brain injuries. We present our preliminary experience.

\section{Methods}

Virtual visits are scheduled at 30-days, 3-months, and 12-months after patients are discharged to a longterm acute care (LTAC) facility. The first PREVAIL patient was seen in December of 2020. These collaborative visits are led by acute care nurse practitioners and involve multidisciplinary input from a neurointensivist and ICU pharmacist in coordination with the LTAC nursing staff, physical and occupational therapists, as well as, the patient.During these visits we review the patient's ICU course with them, filling in gaps in memory and answering questions regarding their care. With the assistance of the LTAC staff we perform a thorough neurological assessment and collect data using screening tools for depression, anxiety, PTSD, global health, physical and cognitive function. Medication reconciliation and coordination of care are also part of this effort.

\section{Results}

To date 23 patients with a mean age of $57+19$ yrs. have been seen in follow up. Ninety percent of scheduled patients have been seen. Acute stroke and seizures were the most common reason for ICU admission. Thirty-day median (IQR): GCS 10.5 (7), NIHSS 21 (11.75), mRS 5 (0.5), PROMISE-10 global health-physical 4th (4) percentile, PROMISE-10 global health-mental 5th (10.25) percentile. Medication changes, neuroimaging, and care coordination were the most commonly performed interventions. Long-term follow up is ongoing.

\section{Conclusions}

A virtual clinic following NeurolCU admission is feasible and results in low rates of missed appointments. Multiple clinically relevant interventions in patient care have been recorded. A better understanding of outcomes and post-intensive care syndrome in this patient population will be gained with long-term follow up. 


\title{
Poster 150
}

\section{Psychological Attachment Orientations and Long-term Post-traumatic Stress Symptoms Among Family Members of Neuro-intensive Care Unit Patients}

\author{
Qiang Zhang, Andrea K. Knies, Jolanta Pach, Tara A. Kimbrough, Aida Martinez, Prerak Juthani, \\ Stephanie Tu, Joan K. Monin, David Y. Hwang
}

Yale School of Medicine, Department of Neurology, New Haven, CT, United States

\begin{abstract}
Background \& Purpose
PTSD symptoms are high amongst families of ICU patients following hospital discharge. Adult attachment theory describes how people respond when separated from loved ones; those who have an "anxious" attachment orientation are preoccupied with being psychologically close with their partners. There is growing evidence that a person's attachment orientation correlates with development of PTSD in various settings; however, this has not been investigated among ICU families. The purpose of this study was to determine whether anxious attachment orientation among family members of neurolCU patients predicts PTSD symptoms at 6 months following ICU discharge.
\end{abstract}

\section{Methods}

We enrolled a cohort of family members of patients admitted to a single neurolCU from 7/2018-4/2019 and 8/2019-3/2020. At ICU discharge, in addition to collecting family and patient demographics, we identified each participant's attachment orientation via a standardized Relationship Questionnaire (RQ). Six months after discharge, participants completed the Impact of Events Scale-Revised (IES-R) to measure PTSD symptoms, with a score of $>24$ indicative of PTSD. We conducted univariate and adjusted multivariate analyses to determine if anxious attachment orientation predicts 6 -month PTSD.

\section{Results}

162/412 (39.3\%) eligible respondents participated. 10/34 (29.4\%) participants with PTSD at 6 months had previously reported an anxious attachment orientation via the RQ at ICU discharge, compared to $17 / 128(13.3 \%)$ participants without PTSD ( $p=0.04)$. Participants with PTSD were also more likely to be Hispanic, to have previous experience caring for disabled patients, and to have reported 3 or more family meetings during the ICU stay. In a multivariate model adjusting for these factors, anxious attachment independently predicted PTSD (OR 3.64, 95\% $\mathrm{Cl} 1.35-9.77, \mathrm{p}=0.01$ ).

\section{Conclusions}

Anxious attachment orientation predicts PTSD symptoms among families of neuroICU patients 6 months following patient discharge. Future ICU interventions designed to decrease rates of family PTSD may be higher yield if focused on this high-risk population. 


\title{
Poster 151
}

\section{Red Blood Cell Distribution Width May Be Associated with Mortality in Hospitalized Men with SARS- CoV-2 Infection and Neurologic Symptoms}

\author{
S. Dania Shujaat, Hannah Schwennesen, Elene Tsopurashvili, Brett L. Cucchiara, Monisha A. Kumar
}

\author{
University of Pennsylvania, Philadelphia, PA, United States
}

\section{Background \& Purpose}

The red blood cell distribution width (RDW) is a routinely obtained blood biomarker that is associated with mortality in myriad diseases, including acute SARS-CoV-2 infection and neurovascular diseases. We aimed to determine whether RDW was predictive of mortality in patients with neurological injury and SARS-CoV2 infection.

\section{Methods}

The study was a retrospective, observational study of consecutive patients with COVID-19 infection hospitalized between March 1-May 27, 2020 at three University-affiliated hospitals. A computerized search strategy identified patients with COVID-19 and neurological symptoms warranting neuroimaging during hospitalization. Demographic data, comorbid diagnoses and laboratory values were extracted from the EHR.

\section{Results}

Of 249 patients, 45\% were women, 66\% were black and mean age was $65.6 \pm 15.6$ years. 121 (50.8\%) patients had a neurological symptom at presentation. Encephalopathy $(58.2 \%)$ was the most common diagnosis, followed by ischemic stroke (10\%), seizure (5.6\%), headache $(4.0 \%)$, and intracranial hemorrhage (3.2\%). Median RDW of the total cohort was 15.1\% (IQR 14.0-17.0) which is elevated compared to normative values (11.5\%-14.5\%). RDW was significantly lower in men when compared to women [14.8\% (IQR 13.7-16.8) versus 15.3\% (IQR 14.4-17.1); $p=0.02$ ]. Patients who died had a higher RDW than those who did not (15.8\% [IQR 14.5-17.8) vs. 15.0\% (IQR 14-16.7); $p=0.01$ ]. Male survivors had a median RDW 14.7\% (IQR 13.6-16.4) versus male non-survivors RDW=15.8\% (14.6-18.3); $p=0.003$. RDW was not significantly different between female survivors and non-survivors. Age $(p=0.009)$, hyperlipidemia $(p=0.01), C A D(p<0.001)$ were other risk factors associated with mortality. In stepwise multivariate analysis stratified by sex and including age, CAD [OR 2.97, 95\% Cl (1.20-7.32); $p=0.02)$, HLD, and RDW, RDW remained significant in men [OR per unit $=1.2195 \% \mathrm{Cl}(1.20-1.42) ; p=0.02$ ], but not in women.

\section{Conclusions}

RDW may be associated with mortality in men with SARS-CoV-2 infection and neurological symptoms. This has not been reported previously and warrants further investigation. 


\section{Poster 152}

\section{Reversing Orders for Life-Sustaining Treatments in Neurologic Emergencies: When is it Appropriate?}

Daryl C. McHugh, Benjamin P. George, Matthew T. Bender, Robert K. Horowitz, David C. Kaufman, Robert G. Holloway, Debra E. Roberts

University of Rochester Medical Center, Dept. of Neurology, Rochester, NY, United States

\section{Background \& Purpose}

Patients with Medical Orders for Life-Sustaining Treatment (MOLST), including "Do Not Resuscitate" (DNR) and/or "Do Not Intubate" (DNI), may be candidates for procedural interventions when presenting with acute neurologic emergencies, such as endovascular therapies for acute stroke and craniotomy for hematoma evacuation. Such interventions may limit morbidity and mortality, but typically they require MOLST reversal. We investigated outcomes of patients with MOLST reversal for treatment of neurologic emergencies.

\section{Methods}

We conducted a retrospective chart review from July 1, 2019 to November 30, 2020 of patients with MOLST reversal treated in the NeuroMedicine Intensive Care Unit at the University of Rochester Medical Center. Variables collected include presenting neurologic disease, MOLST reversal decision maker, procedural interventions, and outcomes.

\section{Results}

Seventeen patients were identified. The most common pre-procedural MOLST was DNR/DNI $(n=15 / 17)$, and all 17 pre-procedural MOLSTs were completed by the patient. MOLSTs were reversed by surrogates in $n=14 / 17$ cases. The median time from MOLST completion to MOLST reversal was 570 days. The most common neurologic emergency was ischemic stroke $(n=10 / 17)$. The majority of patients died $(n=9 / 17)$, most commonly after withdrawal of life-sustaining treatments $(n=7 / 9)$, and $n=6 / 17$ patients eventually recovered sufficiently to return home or to assisted living.

\section{Conclusions}

In neurologic emergencies, urgent shared decision making is needed to ensure goal-concordant care, which may increasingly require reversal of existing MOLST orders. Outcomes of patients with MOLST reversal were heterogeneous, emphasizing the importance of deliberate patient-centered care weighing the risks and benefits of each intervention. 
Poster 153

\title{
Safety of Pterygopalatine Fossa Nerve Blocks
}

\author{
JC Spana, G. Carrazana G, A. Beyer A, K. Zamajtuk, KJ Dickinson, C. Smith, CB Maciel, KM BusI
}

University of Florida, Gainesville, FL, United States

\begin{abstract}
Background \& Purpose
Pterygopalatine Fossa (PPF) nerve blocks are used for perioperative pain control in midface or pharyngeal procedures, and hold promise in lowering opioid requirements in various settings, including headache disorders and ear-nose-throat surgeries. However, large-scale data on procedural safety are lacking, leading to hesitancy surrounding this technique and failure to standardize practice. Therefore, the aim of this study was to characterize the safety of PPF-blocks.
\end{abstract}

\section{Methods}

This retrospective chart review comprising adults who received a PPF-block from January 1, 2016 to August 30, 2020 at the University of Florida. The indications included surgical procedures as well as analgesia for non-surgical pain. Data on the status of the subject before, during, and after the procedure was extracted from medical records. Vital signs pre- and post-procedure and rate of complications were collected. Descriptive statistics were calculated for all variables and quantitative variables were analyzed using the paired t-test to detect differences between pre- and post-procedure.

\section{Results}

169 records were identified for inclusion in the study (41\% males, mean age 46.5 years). The most frequent indication for PPF-block was endoscopic sinus surgery (74\%). We examined the frequency of 5 different side effects in the cohort: bleeding at needle site, pain at injection site, oral/lip numbness, numbness at injection site, and visual disturbance. Only 1 subject in the cohort reported post-procedural side effects (generalized numbness). No significant differences were found in heart rate or mean arterial pressure pre- and post-procedure ( $p=0.1987$ and $p=0.4415$, respectively) and pulse oximetry was clinically similar between timepoints (93\%-100\% pre-procedure, $90 \%-100 \%$ post-procedure).

\section{Conclusions}

These results demonstrate that PPF-blocks are likely a safe avenue for pain management in various settings; however, these results need to be demonstrated in a larger cohort, and more data is needed to establish tolerability and efficacy. 


\section{Poster 154}

\section{Shared Decision-Making in Goals-of-Care Meetings for Critically III Neurologic Patients: A Multi-Center Mixed-Methods Study}

Abhinav Prasad, Connie Ge, Kelsey Goostrey, Praewpannanrai Buddadhumaruk Sun, Catherine L. Hough, Jay Steingrub, Douglas B. White, Susanne Muehlschlegel

University of Massachusetts Medical School, Department of Neurology, Worcester, MA, United States

\section{Background \& Purpose}

Shared Decision-Making (SDM) has been recommended by the Institute of Medicine and several critical care societies to improve difficult decisions in intensive care units (ICUs). Yet, for goals-of-care (GOC) decisions, considered the most difficult decision of all, empirical research in medical-surgical ICUs showed that only $2 \%$ of clinician-family meetings contained all SDM elements. No studies have examined the rate of SDM for critically-ill neurologic patients (CINPs). Understanding and quantifying SDM elements in GOC meetings for CINPs may help identify intervention targets. Here, we characterize and quantify SDM elements during real-life clinician-family meetings for CINPS.

\section{Methods}

We applied the validated 10-element Braddock's SDM instrument to 48 audio-recorded GOC clinicianfamily meetings for CINPs at seven U.S. academic medical centers. Two independent coders doubledcoded 16 transcripts, achieving an inter-rater reliability of $>85 \%$. Subsequently, 32 transcripts were single-coded.

\section{Results}

The SDM score (total number of unique SDM elements present per meeting) ranged between 0 to 10 (median=7; IQR 5-8). Only 3/48 (6\%) of meetings contained all 10 SDM elements. Most commonly used SDM elements were: "Discuss uncertainty" (85\%) and "Assess family understanding" (79\%). Least commonly used SDM elements were "Elicit the context of the decision" (27\%) and "Assess the need for input from others" (33\%). All other SDM elements occurred at variable rates: "Explore the context of the decision" (79\%); "Elicit patient values and preferences" (73\%); "Describe treatment alternatives" (71\%); "Discuss the nature of the decision" (63\%); "Discuss the pros and cons of the choices" (63\%); "Discuss the family's role in decision making" (54\%).

\section{Conclusions}

Comparable to non-CINPs, only a small proportion of clinician-family GOC meetings for CINPs contained all SDM elements. We uncovered notable gaps in SDM with crucial elements missing in a large proportion of meetings. Evidence-based interventions to increase SDM for GOC decisions in CINPs are urgently needed. 


\section{Poster 155}

\section{Smartphone Use During Emergency Neurological Simulation Scenarios}

Melissa B. Pergakis, Afrah A. Ali, WanTsu Wendy Chang, Benjamin Neustein, Casey Albin, Aimee Ayesenne, Samuel A. Tisherman, Nicholas A. Morris

University of Maryland Medical Center, Neurocritical Care, Program in Trauma, Baltimore, MD, United States

\section{Background \& Purpose}

Little is known regarding the use of smartphones in clinical practice. We sought to identify the frequency of and indications for smartphone use during emergency neurological cases.

\section{Methods}

In this retrospective review of a prospective, observational, single-center simulation-based study, participants ranging from sub-interns to attending physicians managed three neurological emergencies: acute stroke followed by tissue plasminogen activator-related hemorrhage, viral encephalitis complicated by status epilepticus, and cardiac arrest with status epilepticus. The primary outcome measure was frequency of smartphone use. The secondary outcome was identification of the correct answer sought via smartphone. We evaluated performance on critical action checklists between participants who used and did not use smartphones. Participants were categorized as experts, intermediates, or novices.

\section{Results}

Sixty-seven participants completed 126 simulation scenarios. Smartphones were used in 67 (53\%) simulations. Of participants using smartphones, 61 participants looked up medication doses, 21 participants looked up management guidelines, 6 participants looked up hospital protocols, and 9 participants used smartphones for assistance with a standard scale. Participants found the correct answer only $24 \%$ of the time using smartphones. Experts were less likely to use smartphones than novice or intermediate participants, $32 \%$ vs. $68 \%$ and $59 \%$, respectively, $p<.05$, but performed better. Of novice and intermediate participants, those who used smartphones performed similarly to participants who did not use smartphones (smartphone users' mean score [standard deviation (SD)] = 12.0 (3.2) vs. non-smartphone users' mean score (SD) = $12.2(3.8), p=.19)$.

\section{Conclusions}

Smartphone use was common in the care of patients with neurological emergencies but did not confer improved clinical performance likely due to incorrect utilization of resources. Participants most likely to use smartphones are less experienced and unable to correctly identify clinical solutions using their smartphones. A comprehensive evidence-based smartphone application for clinical use during emergency neurological situations may improve performance among non-expert clinicians. 


\title{
Poster 156
}

\section{Stroke Mimics: A Systematic Review}

\author{
Abdul Rub Hakim Mohammed, Abilash Muralidharan, Mohammed Numaan Ahmed, Amy G. Rockferry \\ Emergency Medicine, Kokilaben Ambani Hospital and Research Institute, Mumbai, India
}

\section{Background \& Purpose}

Stroke is a common disease with a lifetime prevalence of 1 in 4 in the general population. Stroke-like symptoms may develop in a number of medical conditions commonly referred to as "stroke mimics". The diagnosis of acute stroke is not always straight forward. It is essential to consider stroke mimics in the differential diagnosis of an acute suspected stroke to avoid inappropriate use of expensive, potentially harmful medications and to treat causative conditions.

\section{Methods}

A preliminary search was conducted for papers published in PubMed, Scopus, Web of Sciences, OvidSP and Google Scholar, by searching term stroke mimic, which returned 1127 papers, of which 114 met the criteria. Final paper inclusions were determined by two raters. Data were extracted on: sample size, age and sex of patients; stroke, stroke mimic prevalence; diagnostic method; setting; symptom presentation of strokes mimics; most common stroke mimic diagnosis; and thrombolysis administration. Systematic review analysis was performed. Independent sample t-tests conducted between groups and chi-squared tests compared sex and symptom proportions.

\section{Results}

Approximately half of the studies were prospective and others are retrospective with mostly descriptive aims. The most frequent study setting was emergency departments $(n=40)$. Median age of patients was $69.1 \pm 27.4$ years, and were mostly male (71.2\%). Among suspected acute stroke cases, $8.8 \%$ were stroke mimics. Most common causes were epilepsy (20.4\%), neuropathy (15.3\%), migraine with aura ( $12.4 \%$ ) and hypoglycemia (10.9\%). Outcomes were survival to hospital discharge for $91.2 \%$ and death for $8.8 \%$. Clinical results were significantly different between stroke mimics and the stroke group for low systolic blood pressure, history of diabetes, and no arrhythmia.

\section{Conclusions}

Frequency of stroke mimics in suspected acute stroke is $8.8 \%$, and the most common cause is epilepsy. In order to distinguish stroke mimics, it is useful to understand common diseases presenting as stroke mimics and evaluate clinical features different from stroke by history, clinical examination and investigations. 


\section{Poster 157}

\section{The Neurorecovery Clinic: Design of a Novel Interdisciplinary Post-Neurointensive Care Unit}

Julia M. Carlson, Kristi Emerson, Galina Gheihman, Michael Young, Megan Barra, Jessica Ranford, Kristin Parlman, Julie Maggio, Audrey Cohen, Jennifer Freeburn, Ethan Lester, Christina Maheras, Emily Lewis, Nicole Mazwi, Eric Rosenthal, Daniel B. Rubin, Brian Edlow, Haitham Alabsi, Leigh Hochberg, W. Taylor Kimberly, David J. Lin

Massachusetts General Hospital, Division of Neurocritical Care, Department of Neurology, Boston, MA, United States

\section{Background \& Purpose}

Intensive care unit (ICU) post-discharge clinics have been successful interventions to identify and address sequela of ICU stays, improve care coordination, and reduce staff burn-out. We sought to redesign a single-center neurorecovery clinic for patients after neurointensive care. Here we describe the neurorecovery clinic's mission, structure, and current patient population. We also present provider satisfaction survey results.

\section{Methods}

Patients were referred for follow-up with the neurorecovery clinic by a neurorecovery nurse on ICU rounds. Weekly clinic staff include seven neuro-intensivists, a neurorecovery fellow, nurse, practice manager, and patient services coordinator. One clinic monthly features an integrated multidisciplinary session for patients with complex recovery needs incorporating multiple providers, pharmacists, and therapists. We retrospectively assessed encounters and no-show rates over 8 months (July 2020-March 2021) and cross-sectionally surveyed satisfaction among staff using a confidential online survey distributed by email in April 2021.

\section{Results}

250 encounters were completed during the evaluation period; 23 (9\%) were in person encounters. $47 \%$ of monthly visits were new patient encounters with a mean monthly no-show rate of $10 \%$. Three interdisciplinary visits were completed to date. The most common diagnoses were ischemic stroke (25\%), traumatic brain injury (16\%), and COVID-19 (11.7\%). Provider satisfaction survey results (64\% response rate) indicated 11 (71\%) respondents felt clinic increased job satisfaction, 12 (85\%) found clinic worthwhile, and 12 (85\%) felt clinic improved their sense of purpose. Twelve providers (85\%) reported navigating resource limitation to be a frustrating aspect of clinic.

\section{Conclusions}

A redesigned neurorecovery clinic experienced high utilization of telehealth technology due to the COVID-19 pandemic and treated patients with diverse diagnoses. Survey respondents cited increased provider satisfaction with limited frustrations of increased time or paperwork. Emerging challenges include support for additional patient services consistent with challenges navigating resources cited by providers. Future directions include ascertaining associations between longitudinal outcomes and management in neurorecovery clinic. 


\section{Poster 158}

\section{Variability of Prognostication for Critically III Neurologic Patients: A Multi-Center Mixed-Methods Study}

Connie Ge, Addie Goss, Sybil Crawford, Kelsey Goostrey, Praewpannanrai B. Sun, Catherine Hough, Jay Steingrub, Douglas B. White, Susanne Muehlschlegel

University of Massachusetts Medical School, Worcester, MA, United States

\section{Background \& Purpose}

Withdrawal-of-life-sustaining-treatments (WOLST) varies greatly among critically-ill neurologic patients (CINPs) and cannot be solely attributed to patient and family characteristics. Research in general ICUs has shown that clinicians prognosticate with high variability. Little is known about how clinicians prognosticate to families of CINPs, and whether any associations exist between prognostication and WOLST.

\section{Methods}

We analyzed 43 clinician-family meetings audio-recorded at 7 U.S.-centers for 39 CINPs with 88 family members and 27 clinicians. Two investigators qualitatively coded transcripts using inductive methods (inter-rater-reliability $>80 \%$ ) to characterize how clinicians prognosticate. We applied univariate and multivariable multinomial logistic regression to identify predictors of clinicians' prognostication approaches and explore associations between approach and WOLST.

\section{Results}

Clinicians used 4 distinct prognostication approaches: Authoritative (21\%; recommending treatments prior to discussing values\&preferences; Informational (23\%; prognostic disclosure without discussion of values\&preferences); Advisory (42\%; prognostic disclosure followed by discussion of values\&preferences; and Responsive (14\%; asking surrogates about values\&preferences, then prognostic disclosure). Before adjustment, prognostication approach was associated with center $(p=0.0006)$, clinician specialty (Advisory most common in neurointensivists vs. Informational in nonneurointensivists; $p=0.0095$ ), patient age (Advisory in older patients; $p=0.076$ ), diagnosis (Advisory and Responsive for ischemic stroke; $p=0.059$ ), and meeting length (longer for Advisory and Responsive; $\mathrm{p}=0.03)$. After adjustment, only clinician specialty independently predicted prognostication approach $(p=0.027)$. WOLST decisions occurred in $41 \%$, were more likely in older patients $(p=0.059)$, and with more experienced clinicians $(p=0.07)$. Prognostication approach was not independently associated with WOLST ( $p=0.198$ ) due to insufficient power, although Advisory approach was most common (56\%). Assuming cohen's $w=0.3$ (medium effect-size), $80 \%$ power and $\alpha=0.05,122$ clinician-family meetings are needed to achieve statistical significance for prognostication approach.

\section{Conclusions}

We characterized four distinct prognostication approaches, with clinician specialty as an independent predictor. While underpowered currently, our data suggest that appropriately powered studies are warranted in CINPs to examine the role of clinicians' prognostication in WOLST decisions. 
Poster 159

\section{Anti-Epileptic Prophylaxis Improves Early Post-Traumatic Seizure and Mortality Rates in Elderly TBI Population: A Retrospective Case-control Study Using MIMIC Database}

Adam C. Glaser, John Kanter, Myles D. Boone

Dartmouth Hitchcock Medical Center, Department of Anesthesiology, Hanover, NH, United States

\section{Background \& Purpose}

Traumatic brain injury (TBI) is a major cause of morbidity in the United States, and the elderly represent an understudied and increasingly important TBI population. Current guidelines have modest evidence supporting prophylactic anti-epileptic drug (AED) use to prevent chronic epilepsy.

\section{Methods}

The MIMIC database was used to create a dataset of all patients over the age of 65 with a primary exposure of TBI resulting in an ICU admission to a major US trauma center from 2001-2012. The primary outcomes were seizure within the first seven days of admission to the ICU, or death within 1 year.

\section{Results}

Of 1,041 elderly TBI admissions analyzed, 674 received AED prophylaxis and 367 did not. Patients without AED prophylaxis had significantly higher rates of seizure or death within 1-week $(O R=1.21$ $(1.14-1.28), p<0.0001)$, regardless of model adjustment for age, GCS, ICD9 diagnosis code, or Elixhauser comorbidity score.

\section{Conclusions}

This helps fill two gaps in existing literature with focuses on an elderly TBI population, and varying degrees of TBI severity. This supports current guidelines for AED prophylaxis in TBI, and suggests broader recommendations regardless of TBI severity and comorbidities. 


\section{Poster 160}

\section{Automatic Extraction of Marshall and Rotterdam Scores from Brain Imaging Reports}

Sarah Chacko, Victor Torres Lopez, Angelo Olcese, Amber Robinson, Edward Gaiser, Grace Rovenolt, Gabriella Garcia, Kevin Sheth, Emily Gilmore, Bulent Omay, Sam Payabash, Guido Falcone, Jennifer Kim

Yale School of Medicine Department of Neurology, New Haven, CT, United States

\section{Background \& Purpose}

The Marshall and Rotterdam computerized tomography (CT) scores are designed to categorize the severity of traumatic brain injury (TBI). However, for large datasets, these scores are tedious to manually calculate. Building upon our previous work developing a Named Entity Recognition (NER) model (BrainNER) and accompanying classification system (BrainClass), we developed and assessed the accuracy of our deterministic algorithm in extracting information from free text $\mathrm{CT}$ reports according to the Marshall and Rotterdam scoring systems.

\section{Methods}

We created an algorithm using output from BrainNER and BrainClass. To create a training set, we took 47 CT reports from patients enrolled in the Yale Acute Brain Injury Biorepository and manually analyzed each report to generate rules that aided in the functionalization of the Marshall and Rotterdam scores. To validate our models against the test set, we manually scored an additional 111 CT reports, which were then used to evaluate the final performance of the algorithm.

\section{Results}

In our test set, the algorithm correctly scored 100\% (111/111) and 94.6\% (105/111) of CT reports based on Marshall and Rotterdam classifications, respectively. In the CT reports where the hemorrhagic lesion volume was not reported, our algorithm returned two possible Marshall scores. The first score option was based upon midline shift, cistern compression and hemorrhage presence, while the second score option was based on Marshall category VI to capture hemorrhages described, but not measured, that may exceed $25 \mathrm{~cm} 3$.

\section{Conclusions}

Our algorithm, which incorporates our prior work in BrainClass and BrainNER, accurately scores CT reports consistent with the Marshall and Rotterdam scoring systems. Given the high-performance level, our algorithm can be used to score CT reports not only to reduce the variability between manual scorers but to significantly decrease the time and effort required to manually score imaging in large datasets. 


\title{
Poster 161
}

\section{Beta Blockade after Severe TBI Enhances Neurocognitive Recovery by Improving Cued and Spatial Learning as Well as Memory}

\begin{abstract}
Alfonso J. Lopez, Mohamed Elsaadani, Hailong Song, Christina L. Jacovides, Ana Georges, Monisha A.
\end{abstract} Kumar, Lewis J. Kaplan, Douglas H. Smith, Jose L. Pascual

Perelman School of Medicine at the University of Pennsylvania, Philadelphia, PA, United States

\section{Background \& Purpose}

Neuroinflammation and cerebral edema impair cognitive recovery following severe TBI. Propranolol, a non-selective $\beta$-adrenergic receptor blocker (BB) improves survival after TBI, by blunting neuroinflammation. We hypothesized that BB treatment after TBI improves global cognitive recovery by mitigating deficits in learning and memory.

\section{Methods}

CD1 male mice $(n=30)$ were randomized to severe TBI (controlled cortical impact: $3 \mathrm{~mm}$ diameter impounder, $6 \mathrm{~m} / \mathrm{sec}$ velocity, $1 \mathrm{~mm}$ depth) or sham craniotomy/uninjured followed by intraperitoneal injection of saline or propranolol $(1,2$ or $4 \mathrm{mg} / \mathrm{kg}) \mathrm{q} 12 \mathrm{~h} X 48 \mathrm{~h}$. Learning and memory behavior were assessed by Morris Water maze (MWM) testing days 6-14 post-TBI (D6-14) exploring exercises related to cued learning (platform visible), spatial learning (platform invisible, spatial cues present) and memory (platform removed, spatial clues present). Animal's recovery was further assessed by twice daily body weight loss and the Garcia Neurological Test (GNT: motor, sensory, reflex, balance assessments). Intergroup differences were assessed by Kruskal-Wallis testing $(p<0.05)$.

\section{Results}

Without dose dependence, BB treatment reversed learning and memory deficits induced by TBI to sham (uninjured) levels. Particularly, cued learning (D7 swim distance $-1 \mathrm{mg} / \mathrm{kg}: 302.0+/-33.9 \mathrm{~cm}$, $2 \mathrm{mg} / \mathrm{kg}: 354.6+/-53.2 \mathrm{~cm}, 4 \mathrm{mg} / \mathrm{kg}: 267.0+/-24.2 \mathrm{~cm}$ vs. untreated:806.9+/-81.2, $\mathrm{p}<0.001$ vs all), spatial learning (D12 swim distance - 1mg/kg:368.7+/-27.0cm, 2mg/kg:293.4+/-24.3cm, 4mg/kg:313.9+/$24.9 \mathrm{~cm}$ vs. untreated:546.4+/-23.1, $\mathrm{p}<0.01$ vs all) and memory (D12 swim distance $-1 \mathrm{mg} / \mathrm{kg}: 435.4+/-$ $13.2 \mathrm{~cm}, 2 \mathrm{mg} / \mathrm{kg}: 573.0+/-10.4 \mathrm{~cm}, 4 \mathrm{mg} / \mathrm{kg}: 530.1+/-43.9 \mathrm{~cm}$ vs. untreated:885.6+/-90.1, $\mathrm{p}<0.01 \mathrm{vs}$ all). Weight loss recovery was significantly improved by 2 and 4 but not $1 \mathrm{mg} / \mathrm{kg}$ doses D1-14. GNT scores were also improved with higher but not $1 \mathrm{mg} / \mathrm{kg}$ doses on D3-5.

\section{Conclusions}

BB treatment after severe TBI improves neurocognitive recovery regardless of studied dosage. This improvement relates to memory recovery and enhanced cued and spatial learning. These functional effects are accompanied by improved body weight preservation and integrative neurologic functional assessments. These results support investigation of potential cognitive improvement after BB treatment for TBI patients. 


\title{
Poster 163
}

\section{Incidence of Early Post-Traumatic Seizures in Patients Receiving Prophylactic Levetiracetam}

\author{
Evan Sawyer, Lori Wetmore, Serena Dine, Emily Miller
}

Indiana University Health, Indianapolis, IN, United States

\section{Background \& Purpose}

Early post-traumatic seizures (PTS) are complications within 7 days following severe traumatic brain injury (TBI), occurring clinically in up to $12 \%$ of patients and up to $25 \%$ on electroencephalogram (EEG). Levetiracetam (LEV) is often utilized to reduce early PTS for its safety profile and linear renal elimination. Augmented renal clearance (ARC) is a common ICU phenomenon that has been shown to reduce concentrations of renally eliminated medications, including LEV. The purpose of this study was to determine if increasing frequency of dosing every 8-hours $(\mathrm{Q} 8 \mathrm{H})$ reduces seizures compared to standard dosing every 12-hours (Q12H).

\section{Methods}

This two-center, retrospective cohort study is comparing severe TBI patients receiving LEV $500 \mathrm{mg}$ Q8H to LEV $500 \mathrm{mg} \mathrm{Q12H.} \mathrm{Early} \mathrm{PTS} \mathrm{are} \mathrm{classified} \mathrm{as} \mathrm{clinical} \mathrm{or} \mathrm{subclinical} \mathrm{(EEG-detected)} \mathrm{seizures} \mathrm{within} 7$ days; seizure tendency defined as epileptiform discharges on EEG. The primary endpoint is the presence of early PTS. Secondary endpoints include seizure or seizure tendency, length of stay, and mortality at discharge. Pre-specified subgroup analyses are being conducted on the primary endpoint.

\section{Results}

At this interim analysis, seizures occurred in 3 of 60 patients in $\mathrm{Q} 8 \mathrm{H}$ group and 1 of 61 patients in Q12H group $(5.0 \%$ to $1.6 \% ; p=0.30)$. No differences were observed in seizure or seizure tendency $(6.7 \%$ to $4.9 \% ; p=0.68$ ), length of stay ( 16.0 to 16.0 days; $p=0.58$ ), or mortality $(30.0 \%$ to $27.9 \% ; p=0.80$ ). Of patients who met ARC criteria, seizures occurred in 0 of 23 patients in the Q8H group and 1 of 15 patients in $\mathrm{Q} 12 \mathrm{H}$ group $(0.0 \%$ to $6.7 \% ; \mathrm{p}=0.21)$. Data collection is ongoing to meet prespecified sample size requirements.

\section{Conclusions}

At the interim analysis, no difference in seizures has been found between Q8H and Q12H. Final conclusions pending ongoing data collection and analysis. 
Poster 164

\section{Low Ascorbic Acid Concentrations in Traumatic Brain Injury Patients: A Potential Target for Intervention}

Stefan W. Leichtle, Michael Strein, Sulaiman Almohaish, Melissa Sandler, Vishal Yajnik, Robert Perera, Matt Halquist, Dennis Rivet, Gretchen M. Brophy

Virginia Commonwealth University, Department of Surgery, Richmond, VA, United States

\section{Background \& Purpose}

Antioxidants, such as high-dose ascorbic acid (AA), have recently been studied in critically ill patients with promising initial results. As a state of critical illness marked by oxidative stress, traumatic brain injury (TBI) may cause AA depletion. Identification of AA depletion in TBI patients would suggest a potential benefit of $A A$ administration. The objective of this pilot study was to determine prevalence and extent of AA depletion in TBI patients within the first 96 hours of hospital admission.

\section{Methods}

This was a prospective, observational, IRB-approved pilot study of TBI patients admitted to our institution between 2019 and 2020. Adult patients with non-penetrating, computed tomography (CT)positive TBI of any severity were eligible for inclusion. Patients with severe polytrauma were excluded to minimize confounding causes of AA depletion. Patients had plasma and AA concentrations drawn within 96 hours of admission for two days.

\section{Results}

There were $32 \mathrm{TBI}$ patients included in our study. Most were male (68.8\%), had a mild TBI with median Glasgow Coma Scale Score of 15 [IQR 14, 15], mean lactate level of $2.43 \mathrm{mmol} / \mathrm{L}$ (SD $\pm 2.39 \mathrm{mmol} / \mathrm{L}$ ), and median injury severity score of 14 (IQR 8,21$)$. The mean AA concentration was $27.22 \mu \mathrm{mol} / \mathrm{L}$ (SD \pm 16.49 $\mu \mathrm{mol} / \mathrm{L}$ ) for the first sample and $24.99 \mu \mathrm{mol} / \mathrm{L}(\mathrm{SD} \pm 23.59 \mu \mathrm{mol} / \mathrm{L})$ for the second sample drawn 24 hours later. Plasma concentration of AA for healthy adults typically range from $50 \mu \mathrm{mol} / \mathrm{L}$ to $100 \mu \mathrm{mol} / \mathrm{L}$. Our results suggest an acute deficiency in AA concentrations in mild TBI patients as compared to normal ranges.

\section{Conclusions}

Patients with mild TBI had a lower AA concentration as compared to published AA concentrations in healthy adults. The results of this pilot study provide essential preliminary data to conduct future clinical trials evaluating high-dose AA in mild, as well moderate to severe, TBI patients. 


\title{
Poster 165
}

\section{Relationship of Cytokine Markers in Chronic Subdural Hemorrhage Patients Following a Traumatic Brain Injury}

David J. Puccio, Yuefang Chang, David O.Okonkwo, Enyinna Nwachuku

\author{
University of Pittsburgh, Dept of Neurosurgery, Pittsburgh, PA, United States
}

\section{Background \& Purpose}

The life expectancy within the US has climbed and the incidence of CSDH is expected to double by 2030. Mortality and functional survival rates of elderly patients with chronic subdural hemorrhage (cSDH) are a concern. There is equipoise on the proper management of cSDH. This study was designed to evaluate the inflammatory process of cSDH and to identify markers for risk of recurrence of cSDH.

\section{Methods}

Under an IRB-approved protocol, we enrolled patients ( $>65$ years of age) who presented with a cSDH with the necessity of a surgical intervention for symptomatic cSDH. At the time of surgery, a sample of the cSDH fluid collection was collected for analysis, processed and stored at $-800 \mathrm{C}$ for future batch analyses. Concentrations of inflammatory markers were analyzed via Luminex Array. Primary endpoints were re-accumulation of the $\mathrm{CSDH}$, mortality and neurological outcome assessed by the Glasgow Outcome Scale (GOS) score at 3 months post-surgery.

\section{Results}

For this pilot study, we analyzed the first 20 prospectively enrolled patients for the pro-inflammatory markers measured in CSDH fluid. The mean age was $77.9 \pm 7.4$ years, with the majority (85\%) presenting after a fall with a headache or gait disturbance as their presenting symptomology. $20 \%$ had reaccumulation of the CSDH and required a re-operation. The 3 highest markers were IL-6, CXCL9 and VEGF. Lower IL-6 values correlated with higher GOS scores and less mid-line shift on the presenting CT scan. Increased CSDH thickness $(\mathrm{mm})$ resulted in higher CXCL9 levels $(p=.04)$. Patients with residual cSDH fluid had higher VEGF levels $(p=.04)$.

\section{Conclusions}

This is the first known analysis of chronic subdural collection from the operative site in relationship to cytokine levels. This pilot study identified 3 markers (CXCL9, IL-6 and VEGF) as markers for CSDH characteristics; however, a larger and more diverse sample size is needed for validation. 
Poster 166

The Epilepsy Bioinformatics Study for Antiepileptogenic Therapy (EpiBioS4Rx): Interim Analysis of early epileptiform and seizure incidence

Paul Vespa, Manuel Buitrago Blanco, Courtney Real, Jesus Ruiz Tejeda, Jerome Engel, Arthur Toga, On Behalf of the EpiBioS4Rx Investigators

David Geffen School of Medicine at UCLA, Los Angeles, CA, United States

\section{Background \& Purpose}

Post traumatic epilepsy (PTE) is a well-known long term consequence of TBI, but the process of epileptogenesis is poorly understood. The Epilepsy Bioinformatics Study for Antiepileptogenic Therapy (EpiBioS4Rx) Study was developed to study this process. NIH sponsored study U54 NS100064.

\section{Methods}

EpiBioS4Rx is a multi-center, prospective, observational study of TBI patients 6-100 years old admitted with a GCS 3-13, and hemorrhagic contusion on imaging. The study protocol features continuous electroencephalographic (cEEG) monitoring in the ICU, CT imaging on admission and MRI at 14 days. Local sites report incidence, location, and characteristics of seizures and epileptiform activity on cEEG.

\section{Results}

202 subjects have been enrolled prior to this interim analysis, mean age 45.2 years (IQR 37.8) and mean admission GCS 8.1 (IQR 8). cEEG monitoring data were available for 182/202 subjects with the following results: 47 (26\%) subjects had epileptiform activity, with $27 / 47$ (57\%) of those having seizures and 30/47 (64\%) having Periodic Discharges (PD), and 10/47 (21\%) subjects having a combination of both PDs and seizures. The location of contusions and the location of epileptiform discharges were multifocal; frontal and temporal lobe were the most common sites for contusions and frontal and temporal regions were the most common sites for epileptiform activity.

\section{Conclusions}

The EpiBioS4Rx study interim analysis shows a $26 \%$ incidence of early epileptiform activity, consisting seizures and/or PD, within our cohort. 


\section{Poster 167}

\section{Use of Tranexamic Acid in Penetrating Brain Injury: Preliminary Data Analysis from a Level I Trauma Center in the South Side of Chicago}

Andrea Loggini, Faten El Ammar, Fernando D. Goldenberg, Christopher L. Kramer, Peleg M. Horowitz, Paramita Das, Susan Rowell, Tanya Zakrison, Christos Lazaridis, Ali Mansour

Neurosciences Intensive Care Unit, Department of Neurology, University of Chicago Medical Center, Chicago, IL, United States

\section{Background \& Purpose}

Despite growing interest in the early use of tranexamic acid (TXA) in traumatic brain injury, applicability and literature in the subgroup of patients suffering from penetrating brain injury (PBI) is limited. The aim of this study is to describe the safety and efficacy profile of TXA in a cohort of civilian patients with PBI.

\section{Methods}

This is a retrospective observation describing the use of TXA in PBIs presented at a level I trauma center over two-year period. Demographics, GCS, coagulation profile, and neuroimaging were reviewed before and after TXA administration. Thrombotic complications, GOSE at discharge, and mortality were noted. Patients who received TXA were compared to a severity-matched group who did not receive TXA ( $n$ TXA).

\section{Results}

Out of 207 PBIs screened, 26 received TXA. 24 patients (92\%) had isolated PBI. Mean age was $28 \pm 4$ yrs. Median GCS-M (IQR) was 4(4). Median time (IQR) from injury to in-hospital TXA administration was 117(39) mins. All patients received $1 \mathrm{gr}$ bolus and $1 \mathrm{gr}$ maintenance of TXA. After TXA, there was a significant increase in fibrinogen level (363 (99) vs. 263 (135), p=0.001) and maximal amplitude on TEG (62 (5.1) vs. 55.2 (12.9), $p=0.009)$. Compared to $n-T X A(n=26)$, neuroimaging was stable in 10/16(62\%) vs. $8 / 15(53 \%)$ of patients. GCS-M was unchanged in $18 / 22(82 \%)$ vs. $16 / 22(73 \%)$ of patients. New coagulopathy (22\%vs.28\%), progression of cerebrovascular injury (38\%vs.30\%) and thrombotic complications ( $11 \% v s .17 \%$ ) were also not different between the two groups ( $p>0.05$ for all). There was no statistical difference in mortality $(58 \% v s .62 \%, p=0.777)$ or GOSE $<4(69 \% v s .81 \%, p=0.336)$ at discharge. Multiple variable logistic regression did not suggest an association between TXA and increased mortality (OR:1.08, $\mathrm{Cl}: 0.25-4.70, \mathrm{p}=0.914$ ).

\section{Conclusions}

Administration of TXA is not associated with harm in PBI and favors a trend toward better GOSE at discharge. Larger prospective randomized clinical trials are needed to clarify the efficacy of TXA in this patient population. 


\section{Poster 168}

\section{A Pilot of Peer Support for Post-Pandemic Burnout}

Aleksandra Yakhkind, Bethany Young, Puneet Gill, Meredith Squitiere, Kelsey Nawalinkski, Atul Kalanuria

University of Pennsylvania, Neurology, Philadelphia, PA, United States

\section{Background \& Purpose}

Burnout among critical care clinicians is prevalent, especially in the context of a global pandemic. Clinician burnout can lead to physiologic and psychosocial detriments, decreased job satisfaction, and adverse patient outcomes. Peer support is an effective approach to promote resilience and mitigate clinician burnout by allowing for safe and therapeutic dialogue between colleagues. The neurocritical care unit (NCCU) at the University of Pennsylvania implemented a peer support program one year after the start of the COVID-19 pandemic. We hypothesize that implementation of a unit-based peer support program will decrease incidence of clinician burnout at 6 months after implementation.

\section{Methods}

A sample of the NCCU nursing, advanced practice provider (APP), Fellow, and Faculty teams volunteered as peer supporters. All peer supporters completed standardized online training. Prior to program implementation, an Institutional Review Board approved wellness survey was distributed to all interdisciplinary team members. The survey collected baseline program intent-to-use information and baseline burnout scores using the Maslach Burnout Inventory-Human Services Survey (MB-HSS).

\section{Results}

Sixty-three of 114 NCCU clinicians (55.3\%) completed the survey. Of these, $60 \%(n=38)$ reported burnout in 1 or more burnout category (emotional exhaustion, depersonalization, and/or personal accomplishment). Nurses, Fellows and Faculty experienced similar burnout rates $(64.9 \%, 66.7 \%$, and $62.5 \%$, respectively), while APPs had the lowest burnout rate (28.6\%). Of the three burnout features characterized by the MBI-HSS, depersonalization was the most common (38.1\%). Interestingly, most respondents $(83.1 \%)$ reported openness to using a peer support program and could see its potential benefit for their colleagues, although many qualitatively reported lack of perceived personal need for peer support.

\section{Conclusions}

Burnout rates in our NCCU, one year into the COVID-19 pandemic, indicated ample need for an intervention to bolster clinician wellness and sustain our unit workforce. Implementation of a peer support program was deemed to be acceptable among survey participants. We plan to re-evaluate burnout scores six months after program implementation. 


\section{Poster 169}

\section{Developing and Maintaining Evidence-Based Stroke Education via Monthly Lecture Series for Nurses at an Academic Comprehensive Stroke Center}

Thuy-Tien T Ho, Elizabeth Gunter, Venkatesh Aiyagari

UT Southwestern Medical Center / Department of Neurology / Division of Neurocritical Care, Dallas, TX, United States

\section{Background \& Purpose}

Background: The Joint Commission requires care providers at Comprehensive Stroke Centers (CSC) to earn at least eight hours of stroke education annually. This requirement ensures that staff are updated on current evidence-based practice guidelines to optimize patient outcomes. Purpose: In 2017, we started a hybrid in-person and virtual (live and recorded) monthly stroke lectures series providing Continuing Nursing Education (CNE) and stroke credit hours. Sessions were recorded using GoToWebinar $^{\circledR}$ and available for current or deferred viewing. Our aim was to analyze attendance at these sessions and changes in the attendance pattern during the COVID-19 pandemic, starting in February 2020.

\section{Methods}

Methods: Data was collected via sign-in registrations and online enrollment. We compared the average number of attendees for the in-person and virtual sessions before and after February 2020.

\section{Results}

Results: A total of 40 lectures on various topics have been provided. Total in-person attendance was 632. A total of 14,734 attended the lectures remotely, of which 1,123 watched live-streamed versions and 12,979 viewed recorded sessions. Prior to February 2020, the average attendance at each session was 42 (22 in-person and 20 remote). Subsequently, average attendance increased to 48 (all remote).

\section{Conclusions}

Conclusion: Our study demonstrates the feasibility of a stroke educational lecture series with options of in-person, live streaming, and delayed viewing of recorded sessions. We were able to seamlessly convert the format to a remote-only paradigm during the COVID-19 pandemic with maintained attendance. This format allows for convenient viewing options, especially by nurses engaged in shift work, and viewer numbers have increased over a 40-month period demonstrating the durability of this paradigm. Our success with this stroke lecture series may serve as a model that can be replicated at other comprehensive stroke centers. 
Poster 170

\section{Development of an EVD Competency for Neuro ICU Nurses}

Sarah Peacock, Amanda Tomlinson, Karen Pratt, Rachel Hannon, Kaylie Yost, W. David Freeman

Mayo Clinic Department of Critical Care Medicine, Jacksonville, FL, United States

\section{Background \& Purpose}

Registered Nurses are responsible for care of patients that have External Ventricular Drains (EVDs) including appropriate set-up and monitoring of intracranial pressure, cerebral perfusion pressures (CPP), knowledge of normal intracranial pressure (ICP) measurements and nursing interventions that can be performed to decrease ICP. We found that in our institution, we had a knowledge gap in caring for EVDs due to high nursing turnover and developed a competency for neuroscience intensive care unit (ICU) nurses.

\section{Methods}

All neuroscience ICU nurses ( $n=108$ ) who care for patients with EVDs were required to complete the competency with an in-person check-off from a neuroscience ICU advanced practice provider or neuroscience ICU physician. The goal of was to demonstrate competence in the set-up and caring for a patient with an EVD to allow for real time feedback with question-and-answer sessions. The competency includes three different sections; one to be able to appropriately care for a patient with an EVD in place, appropriate documentation of ICP and CPP and to ensure the RN understands their role with the insertion and set-up of an EVD.

\section{Results}

Out of the 108 registered nurses who completed the competency, 34 completed the post EVD competency survey. $47 \%$ felt competent caring for a patient with an EVD and $52 \%$ felt they have mastered caring for a patient with an EVD. $100 \%$ of nurses felt that an in-person checkoff was more effective than an online module.

\section{Conclusions}

It is important to have ongoing nursing education for high-risk nursing devices. We found that in person check-off of EVDs was effective to determine if nurses were competent in caring for high-risk ICU devices such as EVDs compared with online training that had been done in the past. 


\section{Poster 171}

\section{Evaluation of a Virtual Competency Program in a Neuro ICU}

Giuliana Labella, Elizabeth Garrison, Caitlin Harley, Marie Wilson, Maryanne McCarrin, Dennis Caliguri

Jefferson Health: Jefferson Hospital of Neuroscience, Nursing Department, Philadelphia, PA, United States

\section{Background \& Purpose}

Providing meaningful and effective annual competency-based education to clinical nurses is a constant challenge as due to rotating shift schedules and non-work related obligations. Despite the increasing use of virtual learning within nursing education, its use in competency assessment and education for clinical nurses has not been explored. Modern teaching methods to promote critical thinking, problem solving and information retention related to neurocritical care nursing were applied through virtual skill sessions.

\section{Methods}

The education team distributed a needs assessment to the NICU nursing staff. The survey results were utilized to develop competency content for virtual skills days. The competencies focused on a combination of requirements from regulatory bodies, neurocritical care interventions and high risk low volume topics. Nursing staff had the option to validate competency through specific demonstration of daily work or attend a virtual session. Sessions were live and utilized case scenarios to foster an interactive and collaborative environment that allowed participants to demonstrate competency on all topics. Participants evaluated the virtual learning program in terms acc acceptable method for reinforcement of neurocritical care concepts and their ability to learn virtually.

\section{Results}

110 Neuro ICU nurses participated in virtual competency skills sessions, representing $100 \%$ participation of nursing staff in a 40 bed NICU. The majority of the nurses had greater than 10 years experience (31.8\%). 30\% of nurses had less than 2 years experience. The majority of nurses felt virtual learning would improve nursing practice (mean 4.5 out of 5). Nurses felt they learned from a virtual platform and learning virtually was engaging ( 4.4 and 4.5 out of 5 , respectively).

\section{Conclusions}

Virtual competency assessment appears to be a well received and effective means of maintaining competency. Further research should be pursued to determine whether virtual competency assessment should be the gold standard. 
Poster 172

\section{Implementation of a Nurse-Driven Pain and Sedation Protocol in a Neuroscience Intensive Care Unit}

Naira Arellano, Beth A. Staffileno, Nicolas Panos

Rush University Medical Center, Department of Neurological Sciences, Chicago, IL, United States

\section{Background \& Purpose}

Medications used for pain control and sedation are essential for the care of critically ill adults. National guidelines recommend using a protocol-based approach to manage pain and sedation in the intensive care unit (ICU). A nurse-driven pain management and sedation protocol (RN PAS protocol) for mechanically ventilated patients was implemented in three ICUs at a large midwestern academic medical center. The primary goals of protocol implementation were to decrease time on continuous intravenous sedation (CIS), duration of mechanical ventilation (MV), and ICU and hospital length of stay (LOS).

\section{Methods}

A Plan-Do-Study-Act framework guided implementation. Pre-implementation chart reviews from a historical cohort were conducted to obtain baseline outcomes data associated with pain control and sedation. A protocol algorithm was developed with feedback from key stakeholders, integrated into the electronic medical record and brief education was provided to ICU staff. Post-implementation chart reviews were conducted to evaluate the impact of the protocol on the clinical outcomes and incidence and days of delirium. Percent change was calculated to determine the impact of pre-post differences.

\section{Results}

A total of 41 patients were included, with 26 patients and 15 patients in the pre- and postimplementation groups, respectively. Uptake of the protocol was greatest in the neuroscience ICU. Time on CIS decreased by $47 \%$. Duration of MV, ICU and hospital LOS decreased by $7.2 \%, 16.9 \%$ and $10.8 \%$, respectively. Delirium incidence and delirium days decreased by $17 \%$ and $60.2 \%$ respectively. Fentanyl use and intermittent intravenous benzodiazepine use was higher, use of propofol and dexmedetomidine was lower.

\section{Conclusions}

The RN PAS protocol reduced time on CIS, duration of MV and ICU and hospital LOS. Results support the use of a standardized nurse-driven protocol for pain management and sedation. Implementation was feasible in a neuroscience ICU and led to improvements in clinical outcomes. 


\title{
Poster 173
}

\section{Improving ComfortwWith Conducting End of Life Discussions in the Neuro-ICU: An Educational Intervention}

\author{
Samuel L. Carter, Craig A. Williamson, Venkatakrishna Rajajee, Jade E. Kobayashi \\ University of Michigan Department of Neurosurgery, Ann Arbor, MI, United States
}

\section{Background \& Purpose}

Physician trainees in Neurosciences Intensive Care Units (Neuro-ICUs) rarely receive formal education in discussing devastating neurologic injury with patient proxies, despite often serving as first point-ofcontact in these settings. Trainees feel inadequately prepared to engage in end of life and goal-setting discussions with families. Using a combination of simple didactic education and case-based roleplay, we investigated if a brief educational intervention could provide trainees improved confidence in conducting end of life discussions.

\section{Methods}

Study participants included 18 residents from the departments of Neurosurgery, Anesthesiology, Neurology, Oromaxillofacial surgery, \& Otolaryngology rotating in the Neuro-ICU, as well as 11 medical students. The educational intervention consisted of two one-hour sessions held in small groups, with use of the SPIKES (Setup, Perception, Invitation, Knowledge, Emotion, Strategy/Summary) protocol taught as a conversation tool. Direct participation from trainees was a key component of the education. Paired samples t-tests were used to evaluate pre- and post-intervention surveys.

\section{Results}

Preliminary analyses suggested that this educational intervention led to a two point average (on a 5point Likert scale) increase in confidence across multiple metrics, including but not limited to: using a systemic approach when leading end of life discussions $(t=2.81, p<.001)$, disseminating neurologic prognosis to family members $(t=1.95, p<.001)$, and managing emotionally-charged situations $(t=2.23$, $p<.001)$. Qualitative feedback indicated that adaptation of the SPIKES protocol would be particularly useful in respondents' future goals-of-care conversations with patients and proxies. Respondents also indicated that roleplaying sample cases was a key component in development of necessary conversation skills.

\section{Conclusions}

Our findings suggest that brief educational courses such as described above could fill a critical education gap, and be feasibly incorporated into formal Neuro-ICU training. 
Poster 174

Intermittent Straight Catheterization Instead of Continuous Indwelling Catheterization is Associated with a Reduction in Catheter Associated Urinary Tract Infection Rate in Neurological ICU Population

Pravin George, Christopher R. Newey, Brittiany Ochsner

Cleveland Clinic, Neurology/Neurosciences Critical Care, Cleveland Clinic, Cleveland, OH, United States

\section{Background \& Purpose}

Indwelling urinary catheters (IUC) are essential for care in the neurological critical care unit (NCCU). Acute urinary retention (AUR) often affects neurologically injured patients and remains a leading reason for IUC. Most of the catheter associated urinary tract infections (CAUTIS) in our center were related to catheters utilized for AUR. In an effort to reduce CAUTI, our NCCU switched to an intermittent straight catheterization (ISC) strategy for patients with AUR.

\section{Methods}

Intervention: NCCU teams were educated late 12/2018 regarding an IUC policy change instituted on $1 / 1 / 2019$. Instead of IUC, patients with AUR underwent q6h to q8h ISC with bladder ultrasound scans. IUC use was discouraged unless urological trauma was noted, or anatomy was inconsistent or did not allow for around the clock ISC. Pharmacotherapies for AUR (e.g. bethanechol, doxazosin, tamsulosin) were encouraged, as appropriate. Data and Statistics: January 2017 and December 2018 (preintervention) and January 2019 and December 2020 (post-intervention) CAUTI events and IUC days were analyzed. CAUTI definition and rates were calculated per National Healthcare Safety Network method by dividing the total number of CAUTI episodes within a specific time period by the total number of catheter days within the same time period multiplied by 1000. A comparison of incidence rates was completed using MedCalc.

\section{Results}

Pre-intervention CAUTI rate was calculated as 2.89 (19 events / 6563 catheter days x 1000) and postintervention rate was calculated as 1.24 (7 events / 5631 catheter days $\times 1000$ ). This calculated to a $43 \%$ reduction in the post-intervention time period when compared with pre-intervention $(p=0.0489)$, incidence rate ratio 2.3288 .

\section{Conclusions}

AUR in the NCCU is a common reason for IUC utilization and due to duration of catheter use can lead to CAUTI. An intervention utilizing ISC instead of IUC for patients with AUR in the NCCU was associated with a significant decrease in CAUTI rate. 


\title{
Poster 175
}

\section{Intubation Checklist impacting Staff Education and Satisfaction}

\author{
Amanda Tomlinson, Joshua. Propst, Edward Blair, Trever Rosenbush, Philip Lowman
}

Mayo Clinic Critical Care Department, Jacksonville, FL, United States

\section{Background \& Purpose}

Intubation in the intensive care setting is a frequently performed procedure however little standardization is present among providers and often performed by a variety of skilled providers. In our institution we found this inconsistency to be causing dissatisfaction among our nursing staff. In response, we developed a simple, easily accessible checklist (Figure1) that could be utilized during intubations.

\section{Methods}

In a mixed intensive care a pre intervention survey was formulated and distributed (Attending/consultant (7, 33.3\%), APP (10, 47.6\%), Fellow (4, 19.0\%), and Respiratory therapist (0, 0.0\%), Nurse $(21,75.0 \%)$, not identifying role $(7,25 \%))$ totaling 49 responses. After reviewing the answers and comments from each survey we felt there was a place for improvement. The goal was to increase staff satisfaction and education with urgent and elective intubations by providing a checklist for the intubation process. Education was disseminated and the checklist was attached to each of the premade soft pack kits used in each intubation.

\section{Results}

After initiation of checklist a post survey was issued (Attending/consultant (6, 46.2\%), APP (7, 53.8\%), and Nursing $(9,52.9 \%)$, Respiratory Therapy $(8,47.1 \%)$ totaling 32 responses. $88.9 \%$ of the non-provider group felt the intubation checklists could improve patient safety and $82 \%$ of the providers reported the checklist streamlined the process and reduce the total time to intubation.

\section{Conclusions}

After implantation there was noted perceived benefit by the staff. However while not quantifiable the staff comments regarding the implementation of the checklist demonstrated a favorable increase in satisfaction. We felt that the accessibility and simplicity made the utilization of the intubation checklist a success. Overall we found utilizing the intubation checklist to require minimal effort but yielded favorable results. 


\section{Poster 176}

\section{Neurocritical care services in Australia: A nation-wide survey}

\section{Xiuxian Pham, David Pilcher, Edward Litton, Patrick Kwan, Piero Perucca, Andrew Udy}

School of Public Health and Preventive Medicine, Monash University, Melbourne, Victoria, Australia

\section{Background \& Purpose}

Neurocritical care (NCC) is a rapidly developing field within intensive care medicine. However, NCC implementation is variable worldwide and remains an evolving area in Australia. This study aimed to survey intensive care units (ICUs) in Australia to determine the availability of NCC services.

\section{Methods}

An electronic questionnaire was distributed via the Australian and New Zealand Intensive Care Society (ANZICS), between August and October 2020, with each ICU in Australia invited to participate. Survey questions included admission characteristics, availability and access to NCC services, and use of neuromonitoring devices. De-identified data were analysed using descriptive statistics. The Alfred Hospital HREC provided ethics approval (Ref 289/20).

\section{Results}

Seventy-eight of 175 (44.6\%) ICUs participated in the survey. 34.6\% (27/78) of ICUs did not routinely treat acute brain injured patients. Of those that did $(n=51)$, only 4 centres reported specialising in NCC, while 9 employed an intensivist subspecialising in NCC. A neurosurgical unit was present in $44.9 \%$ $(35 / 78)$ of hospitals. $35.9 \%(28 / 78)$ of hospitals did not have a neurology unit, and $15.4 \%(12 / 78)$ had access to a consulting service only. $76.9 \%$ (60/78) did not have access to a dedicated epilepsy service. Of the ICUs that routinely treated brain injuries, intermittent electroencephalography, ICP monitoring and extra-ventricular CSF drainage were used in 82.4\% (42/51), 76.5\% (39/51), and 76.5\% (39/51), respectively. Brain tissue oxygen monitors and optic nerve sheath diameter assessment were only used in $2 \%(1 / 51)$.

\section{Conclusions}

Although two-thirds of ICUs providing survey data treated acute brain injured patients, access to specialised neurocritical care, neurosurgical and/or neurology services was highly variable. Moreover, utilisation of advanced neuromonitoring devices was limited. Further evaluation is necessary to identify if patient outcomes improve in centres with greater access to neurocritical care services. 


\section{Poster 177}

\section{Nurse-Driven Interprofessional Rounds: Impact on Team Satisfaction and Communication}

Justin Daniels, Haley Miranda, Sanjeev Keshary, Angie Ballew, Walter Orr, Niaman Nazir, Aaron LacKamp

The University of Kansas Health System Anesthesia and Critical Care, Kansas City, KS, United States

\section{Background \& Purpose}

Poor communication has been shown to be a major contributor to the estimated 250,000 patient deaths/year from medical errors. Adverse events may be correlated with dissatisfied bedside nurses and poor hospital work environments. Nurse-driven interprofessional rounding may enhance communication between care team members, improve the work environment for nurses, and increase team satisfaction, ideally contributing to better patient outcomes.

\section{Methods}

We implemented a nurse-driven rounding format. ICU nurses were responsible for presenting relevant systems-based data for patients. A multidisciplinary team then discussed the daily plan of care. A scripted rounding sheet following an organ systems approach was created for nursing handoffs. Education occurred during the 3 months prior to the change. Pre- and post-intervention surveys were distributed via RedCAP to ICU nurses, advanced practice providers (APPs), and physicians, all of whom work primarily in the neurosciences ICU. Post-intervention surveys were disseminated 8 months after implementation.

\section{Results}

Bedside nurses provided the majority of responses (75\%). Few items in our pre- and post-survey results reached statistical significance. While we saw a trend toward improved relationships and communication between team members, with nurses reporting they felt physicians and APPs demonstrated greater respect for the role of the beside nurse in patient care, we also saw a trend toward overall decreased nursing satisfaction. This finding may be related to external factors such as the pandemic and the format of multi-team rounding.

\section{Conclusions}

While we did not demonstrate statistically significant improvements in communication and teamwork, we identified factors that made implementation of nurse-driven rounding difficult in our setting. Challenges included a multi-team ICU, nursing workflow, and stress on the unit with the concurrent pandemic. With higher levels of pandemic-associated burnout in healthcare workers, further studies investigating the impact of nurse-driven rounds and associated effects on medical errors and nursing retention may be worthwhile. 
Poster 178

\title{
Proposal for Tiered Neurocritical care Advanced Practice Providers Advancement through Annual Educational and Procedural competence pathway
}

\section{Anusha Battineni, Naresh Mullaguri, Ryan Hakimi}

Prisma Health Upstate University of South Carolina School of Medicine, Medicine, Greer, SC, United States

\begin{abstract}
Background \& Purpose
Neurocritical care is evolving rapidly. Advanced practice providers (APPs) play a vital role in neurocritical care units in providing excellent patient care. APPs come from various training backgrounds and working experiences. Ensuring continuous neurocritical care education and optimal procedural performance for APPs are vital for delivering safe and effective patient care. So far there are no published data to guide the initiation of such programs. We are proposing a tiered approach for APPs working in neurocritical care units for the maintenance of educational and procedural competence. Neurocritical care society (NCS) offers excellent educational resources for clinicians and APPs with programs like PONS, NCS podcast, and NCS on-call. We believe structured annual neurocritical care-related educational activities utilizing NCS resources along with documented procedural competence help APPs to advance in their career and also help deliver excellent evidence-based care for neurocritical care patients.
\end{abstract}

\section{Methods}

Tier 1 competencies include completion of Emergency neurological life support, advanced cardiac life support, National Institute of Health stroke scale, modified Rankin scale certification renewed every 2 years. Tier 2 includes Tier 1 certifications along with procedural competencies. Performing 10 central lines, 10 arterial lines, 4 endotracheal intubations, and 5 lumbar punctures with no periprocedural complications were proposed for reaching Tier 2 level. Tier 3 constitutes a minimum of 2 years of neurocritical care APP experience after the completion of the orientation period and meeting annual procedural competencies listed in tier 2 along with completion of annual educational competencies from NCS including 5 PONS credits, 5 NCS podcasts, and 5 neurocritical care on-call chapters.

\section{Results}

N/A

\section{Conclusions}

A tiered annual competency-based approach for advanced practice providers working in neurocritical care service is critical to delivering safe and effective patient care. By ensuring the completion of annual educational, procedural competencies, and renewal of essential certifications, APPs can be advanced in their career. 


\section{Poster 180}

\section{A Simple Score to Predict Intracerebral Hemorrhage Risk in Non-vitamin K Antagonist Oral Anticoagulant Users}

Alvin S. Das, Robert W. Regenhardt, Elif Gökçal, Mitchell J. Horn, Andrew D. Warren, Ugur Gurol, Alessandro Biffi, Joshua N. Goldstein, W. Taylor Kimberly, Christopher D. Anderson, Anand Viswanathan, Lee H. Schwamm, Steven M. Greenberg, Jonathan Rosand, M. Edip Gurol

Department of Neurology, Massachusetts General Hospital, Harvard Medical School, Boston, MA, United States

\section{Background \& Purpose}

Current risk prediction bleeding scores fail to predict intracerebral hemorrhage (ICH) with high accuracy in non-vitamin $\mathrm{K}$ antagonist oral anticoagulant (NOAC) users. Herein, we develop a risk prediction score based on clinical risk factors and MRI biomarkers.

\section{Methods}

Clinical/radiological data were collected from consecutive atrial fibrillation patients on NOACs with ICH (NOAC-ICH) and without ICH from 2017 to 2020. The frequency/topography of MRI biomarkers in both cohorts were assessed. Baseline demographics and neuroimaging markers were compared in univariate tests between both groups. The variables were then weighted based on $\beta$ values of univariate tests. Significant associations $(p<0.05)$ were entered into a multivariable regression model to determine factors that predict the development of $\mathrm{ICH}$.

\section{Results}

53 NOAC-ICH patients and $94 \mathrm{ICH}$-free NOAC users were included. Diabetes, any smoking, and antiplatelet usage were more common in $\mathrm{NOAC}-\mathrm{ICH}$ than $\mathrm{ICH}$-free patients. Severe white matter hyperintensities (WMHs), lacunes, deep lacunes, cortical superficial siderosis (cSS), and cerebral microbleeds (CMBs) were more common in the $\mathrm{ICH}$ cohort than the $\mathrm{ICH}$ free cohort. When entered into a multivariate regression model, deep lacunes and severe WMHs were not significant. Ultimately, diabetes (1), any smoking (1), antiplatelet usage (1), CMBs (2), and cSS (3) comprised the final hemorrhagic risk score. Using this system, a score of 2 provided a sensitivity and specificity of $88 \%$ for predicting the development of $\mathrm{ICH}$.

\section{Conclusions}

In this study, we demonstrate a simple score that predicts the development of ICH by incorporating both risk factors and neuroimaging markers. Although screening MRIs are not currently performed prior to initiating NOAC therapy, these data suggest that patients of high-hemorrhagic risk may be identified. 


\title{
Poster 181
}

\section{Aggressive Blood Pressure Reduction in the First 72 Hours after Spontaneous Intracerebral Hemorrhage is Associated with Poor Discharge Outcome}

\author{
S. Haldal, E. Green, K. Nalleballe, M. Zaghlouleh, K. Patrice, V. Shah
}

University of Arkansas for Medical Sciences, Department of Neurology, Little Rock, AR, United States

\section{Background \& Purpose}

Aggressive and rapid blood pressure control after intracerebral hemorrhage (ICH) has been regarded safe and standard-of-care for decades, however detrimental effects of rapid blood pressure reduction are increasingly being recognized. We conducted this study to assess the relationship between blood pressure thresholds and discharge outcomes after $\mathrm{ICH}$.

\section{Methods}

We conducted a retrospective analysis on a cohort of spontaneous $\mathrm{ICH}$ patients admitted to a tertiary hospital in Arkansas. Demographics, ICH severity variables and blood pressure (SBP/MAP) recordings in the first 72 hours of hospitalization were extracted. Outcome variables recorded included hospital mortality and discharge disposition. Poor discharge outcome was defined as the nationwide inpatient sample outcome measure or NIS-SOM, which is a composite outcome measure comprising death, discharge to hospice, skilled nursing facility, long-term acute care facility, tracheostomy and/or PEGtube placement. NIS-SOM has been validated in SAH and has been shown excellent correlation and high agreement with modified Rankin score of $>3$ (4-6)1. Univariate analysis was conducted to assess factors associated with outcome. Multivariable logistic regression models poor discharge outcome were generated.

\section{Results}

After excluding patients with missing $\mathrm{ICH}$ volume data and patients that either died or were discharged in the first 72 hours, a total of 152 patients were included in the final analysis. Mean age was 64.5 $(+14.2)$ years, median GCS was 13 and $27.2 \%$ had ICH volume $>30 \mathrm{~mL}$. Hospital mortality was $9.4 \%$, $10.3 \%$ were discharged home and $37.6 \%$ had poor discharge outcome. After adjusting for admission GCS, age and ICH volume>30, any SBP reading > $180 \mathrm{mmHg}$ (O.R. [95\% C.I.], 5.16 [1.97-13.49]) as well as any MAP reading $<75 \mathrm{~mm} \mathrm{Hg}$ (12.93 [1.19-139.79]) in the first 72 hours increased odds for poor discharge outcome (ROC area: 0.843 ). Similarly, after adjusting for GCS, age, ICH volume $>30$, every $\mathrm{mm}$ of $\mathrm{Hg}$ drop in SBP in the first 72 hours independently increased odds for poor discharge outcome (OR: 1.019 [1.007-1.032]) (ROC Area: 0.799). Higher the lowest SBP recording in the first 72 hours, higher the odds for being discharged home to self-care (OR per mm Hg SBP: 1.056 [1.010-1.105]). While pneumonia and other in-hospital complications were associated with poor outcome, none of them independently predicted poor discharge outcome in the models.

\section{Conclusions}

In a cohort of $\mathrm{ICH}$ patients in a rural population with a high prevalence of uncontrolled hypertension, blood pressure reduction was significantly associated with worse discharge outcomes and lower likelihood for discharge to home, independent of ICH severity, age and infectious in-hospital complications. Further prospective validation of our findings are needed. 


\section{Poster 182}

\section{An Integrated Hematoma Evacuation/Tissue Preservation System Coupled with Immune Profiling for Evaluation of Neuroinflammation after Primary Supratentorial Intracerebral Hemorrhage}

Jay B. Lusk, Michael P. Smith, Quentin J. Quinones, Janet S. Staats, Kent J. Weinhold, Shahid M. Nimjee, L. Fernando Gonzales, Peter M Grossi, Daniel T. Laskowitz, Michael L. James

Duke University School of Medicine, Department of Neurology, Durham, NC, United States

\section{Background \& Purpose}

To identify novel, disease-modifying treatments for $\mathrm{ICH}$, we need to understand the neuroinflammatory landscape of $\mathrm{ICH}$ that gives rise to significant and long-lasting neuronal damage. Previous work in preclinical models has identified a complex neuroinflammatory network after $\mathrm{ICH}$, but it is not clear how those findings translate to human $\mathrm{ICH}$. Therefore, we integrated a process for hematoma evacuation, tissue preservation and transport, and immune profiling through flow cytometry to directly characterize neuroinflammation in human $\mathrm{ICH}$.

\section{Methods}

We performed a pilot study of seven patients with acute primary supratentorial ICH. Hematomas were evacuated with a minimally invasive approach using the Myriad NOVUS system, which evacuates the hematoma in piecemeal fashion and collects microbiopsy samples. The NICO automated preservation system was then utilized to cryopreserve tissue. Samples were transported to a site located 30 miles from the site of hematoma evacuation and were processed for storage within 6 hours of arrival. Flow cytometry was performed under standardized methods, and results from hematoma samples were compared to results from peripheral blood mononuclear cells (PBMC).

\section{Results}

Patient median age was 58 years (range: 22-60). Five patients survived to discharge. Five patients had spontaneous ICH due to uncontrolled hypertension, one had ICH due to cerebral amyloid angiopathy, and one was later classified as traumatic $\mathrm{ICH}$. No adverse safety events were observed with the use of the hematoma evacuation system. Hematoma samples had a higher portion of natural killer cells and monocytes, a similar percentage of CD8 cells, and lower proportions of CD4 cells and lymphocytes compared to PBMCs.

\section{Conclusions}

This pilot presents successful characterization of ICH neuroinflammatory cell populations using an integrated hematoma evacuation and sampling system followed by flow cytometry after moderate distance transport. Larger-scale investigation using this method may reveal promising new therapeutic targets to improve ICH outcomes. 
Poster 183

\title{
Blood Pressure Variability when Transitioning from Intravenous to Oral Antihypertensive Agents in Intracranial Hemorrhage Patients
}

\author{
Abdulrahman I. Alshaya, Meshari Alghamdi \\ King Saud Bin Abdulaziz University for Health Sciences, College of Pharmacy, Pharmacy Practice Dep, \\ Riyadh, Saudi Arabia
}

\section{Background \& Purpose}

Blood pressure variability (BPV) in patients with intracranial hemorrhage (ICH) is linked with an increased risk of mortality. Intravenous (IV) antihypertensive agents are commonly used to achieve sustained target blood pressure goals; however, this is not a feasible long-term option. The transition process of antihypertensive agents from IV to an enteral route is not well-established yet in patients with $\mathrm{ICH}$. We aim to investigate the BPV of antihypertensive agents during the transition period from IV to enteral route in $\mathrm{ICH}$ patients.

\section{Methods}

This is a retrospective single-center study at a tertiary teaching hospital in Riyadh, Saudi Arabia. Data were extracted from electronic medical records after obtaining IRB approval. Patients were included if they were $>18$ years old, admitted with spontaneous $\mathrm{ICH}$, and had administered antihypertensive agents continuous infusion prior to transitioning to oral agents. The major outcome was average BPV on the first day after the transition. The minor outcome was the incidence of acute kidney injury (AKI) on day 7.

\section{Results}

We screened 175 patients, of which 36 patients were included. An aim of systolic blood pressure goal of $<160 \mathrm{mmHg}$ was documented for $91.5 \%$ of patients. Nicardipine was used in $69.4 \%$ and labetalol in $30.6 \%$ of patients. BPV after the transition was a median of 29 [IQR $34-63$ ] $\mathrm{mmHg}$, and AKI was documented in $25 \%$ of the included patients.

\section{Conclusions}

Our findings demonstrated high BPV during the transition period from intravenous to enteral antihypertensive agents for patients with ICH. Proper attention should be implemented to ensure a safe transition process. More data are needed to optimize BPV in ICH patients. 


\section{Poster 184}

\section{Cardiovascular Predictors of Intracerebral Hematoma Expansion}

Hera A. Kamdar, Khalid Sawalha, Terese Gullo, Sheila Okere, Mohammad Hamed, Archana Hinduja, Omar Hussein

The Ohio State University, Columbus, $\mathrm{OH}$, United States

\section{Background \& Purpose}

There is limited evidence on the effect and relevance of cardiovascular parameters on the cerebrovascular system when an intracerebral hemorrhage $(\mathrm{IC}) \mathrm{hH}(\mathrm{H})$ occurs. While recent studies evaluating this relationship are conflicting, one evaluating the effect of systolic cardiac function on clinical outcomes in ICH patients, found low cardiac ejection fractions to be associated with poor clinical outcomes. Our primary objective was to study such correlations and identify various cardiovascular predictors of hematoma expansion, which in turn may affect clinical outcomes.

\section{Methods}

This is an IRB approved single center retrospective study utilizing The Ohio State University, Wexner Medical Center's institutional Get with the Guidelines-Stroke registry between 2013 and 2017. Patients included were older than 18 years of age, admitted with an acute $\mathrm{ICH}$, and had an echocardiogram during their hospitalization. Univariate and Multivariate Logistical Regression Analysis were used to ascertain cardiovascular predictors of hematoma expansion.

\section{Results}

Two-hundred forty-nine patients were identified from our GWTG-S registry and met inclusion criteria. Of these patients, a history of peripheral arterial disease (PAD) $(p=0.015)$, presence of aortic stenosis (AS) on the echocardiogram $(p=0.025)$, and finally a positive spot sign on the CT-angiogram (CTA) of the head $(p=0.000$ ) were found to be independent predictors of ICH expansion. Our results are validated as the widely-accepted phenomenon of a positive spot sign on CTA predicting hematoma expansion is also significant within our data set. Neither correlation with a history hypertension nor elevated blood pressure on presentation was found to be significant. An interesting inverse relationship with a history of congestive heart failure $(p=0.027)$ was also seen.

\section{Conclusions}

This study highlights novel cardiac predictors of hematoma expansion, including PAD and AS, that should be closely monitored by clinicians when preventing and treating acute hemorrhagic strokes. Larger prospective studies are needed to further investigate such associations to ultimately optimize cardiocerebral health. 


\section{Poster 185}

\section{Clinical Factors and Outcomes Associated with Unplanned Readmissions to the Neurological Intensive Care Unit in Patients with Spontaneous Intracerebral Hemorrhage}

Ruth Tangonan, Ronald Alvarado-Dyer, Andrea Loggini, Faten El Ammar, Christopher L. Kramer, Christos Lazaridis, Fernando D. Goldenberg, Ali Mansour

Neurosciences Intensive Care Unit, Department of Neurology, University of Chicago Medicine, Chicago, IL, United States

\section{Background \& Purpose}

Unplanned readmission to the neurological ICU (b-NCCU) is an under investigated topic in patients admitted after spontaneous intracerebral hemorrhage (ICH). The purpose of this study is to investigate the prevalence, clinical risk factors, and outcome of b-NCCU in a large cohort of patients admitted after $\mathrm{ICH}$.

\section{Methods}

This is a retrospective cohort study analyzing b-NCCU in patients admitted with sICH over an eight-year period. For each patient, demographics, medical history, clinical presentation, length of ICU stay, cause of readmission, and mortality were reviewed. B-NCCU was defined as an unplanned readmission from a general floor service, during the same hospitalization. A multiple variable analysis was used to define independent variables associated with b-NCCU and between b-NCCU and mortality. The significance level was set at $p<0.05$.

\section{Results}

A total of 221 patients were included in the study. Among those, 20 (9\%) had a b-NCCU. Respiratory complications ( $\mathrm{N}=11)$ were the most common reason for readmission, followed by neurological $(\mathrm{N}=5)$ and cardiac $(\mathrm{N}=4)$ complications. Several significant variables were found to be independently associated with readmission: location of hemorrhage in the basal ganglia (OR:3.1, 95\% $\mathrm{Cl}: 1.1-8.5$, $\mathrm{p}=0.03$ ), dysphagia at the time of transfer (OR: 3.8, 95\% Cl:1.0-14.5, $\mathrm{p}=0.04$ ), and use of supplemental oxygen at the time of transfer (OR: $3.3,95 \% \mathrm{Cl}: 1.1-10.0, \mathrm{p}=0.03$ ). In a different multiple variable logistic regression, after correcting for ICH score, readmission to ICU was also independently associated with mortality (OR: 14.1, 95\% Cl:2.8-71.7, $\mathrm{p}<0.01$ )

\section{Conclusions}

B-NCCU is not an infrequent complication in patients with sICH and is associated with higher hospital LOS and mortality. We identified relevant and potentially modifiable risk factors associated with readmission. Future prospective studies are necessary to develop patient-centered strategies that will improve transition from the NICU to the general floor. 
Poster 186

\title{
Clinical Outcomes after Prothrombin Complex Concentrate for Warfarin-Associated Intracerebral Hemorrhage and Baseline Glasgow Coma Scale Less than or Equal to 8
}

\author{
Kristen Koehl, Nicholas G. Panos, Gary D. Peksa, Tara Kimbrough, Hannah Breit, Giles W. Slocum \\ Rush University Medical Center, Chicago, IL, United States
}

\section{Background \& Purpose}

There is limited evidence describing the mortality benefit of utilizing 4-factor prothrombin complex concentrate (4F-PCC) in patients presenting with a warfarin-associated intracerebral hemorrhage (ICH) and a Glasgow Coma Scale (GCS) of $\leq 8$. The aim of this study is to determine the potential mortality benefit of the aforementioned patient population.

\section{Methods}

This was a retrospective chart review, performed at a comprehensive stroke center from October 2013 through August 2020. Patients were included if they were $\geq 18$ years of age, experienced a spontaneous $\mathrm{ICH}$ with baseline GCS $\leq 8$, treated with warfarin prior to admission, had a baseline INR $\geq 1.7$, and received $4 \mathrm{~F}-\mathrm{PCC}$ for INR normalization due to warfarin-associated $\mathrm{ICH}$. The primary outcome was inhospital mortality at 30 days.

\section{Results}

There were 252 patients that received 4F-PCC in the specified time period. Of those patients, 25 patients met inclusion criteria. Sixteen patients (64\%) experienced in-hospital mortality. When compared to an estimated $80 \%$ mortality rate in the studied patient population, there was no statistically significant difference $(p=0.208)$ in mortality when 4F-PCC was utilized to reverse INR.

\section{Conclusions}

The administration of 4F-PCC in patients presenting with warfarin-related ICH and GCS $\leq 8$ did not result in a mortality benefit. Our results are limited by study design and sample size. Thus, larger studies are needed to determine if a benefit exists for 4F-PCC in this patient population. Although the results are not statistically significant, our study suggests that there may be a clinically significant mortality benefit when 4F-PCC is utilized. 


\title{
Poster 187
}

\section{Comparing Hematoma Characteristics In Primary Intracerebral Hemorrhage versus Intracerebral Hemorrhage Caused by Structural Vascular Lesions}

\author{
Joshua Kahan, Hanley Ong, Alexander E. Merkler, Hooman Kamel, Santosh B. Murthy \\ Weill Cornell Neurology, New York, NY, United States
}

\section{Background \& Purpose}

Intracerebral hemorrhage $(\mathrm{ICH})$ caused by structural vascular lesions is associated with better outcomes than primary $\mathrm{ICH}$, but the factors that influence this relationship are poorly understood. We tested the hypothesis that ICH from vascular lesion has more benign hematoma characteristics compared to primary $\mathrm{ICH}$.

\section{Methods}

We performed a retrospective study using data from the Cornell Acute Stroke Academic Registry (CAESAR), which includes all patients hospitalized at our center with ICH between 2011-2017. The SMASH-U criteria were used to adjudicate the etiology of ICH. The co-primary outcomes were admission parenchymal hematoma volume and hematoma expansion at 24 hours. Hematoma volumes were calculated using the 3D Slicer image computing platform and were log-transformed. T-tests, Chi square, linear and logistic regression analyses were performed with adjustment for demographics. Additionally, baseline ICH severity was adjusted for when studying hematoma expansion.

\section{Results}

A total of 231 patients were included of whom 189 (82\%) had primary ICH, while 42 (18\%) had a vascular lesion. Compared to primary ICH patients, those with structural vascular lesions were younger (48 vs. 69 years, $p<0.001$ ), less likely to have hypertension ( $29 \%$ vs. $74 \%, p<0.001)$, had lower admission systolic blood pressure ( $140 \pm 23$ vs. $164 \pm 35, p<0.001)$, less frequently had IVH ( $26 \%$ vs. $44 \%, p=0.03$ ), and had mostly lobar or infratentorial hemorrhages. The mean admission hematoma volume was smaller with vascular lesions (12.4 \pm 2.8 vs. $23.6 \pm 2.2, \mathrm{~mL}$ ). In multiple linear regression models, ICH from a vascular lesion was associated with smaller admission hematoma volume (beta, $-0.7,95 \% \mathrm{Cl},-1.2$ to $-0.1, \mathrm{p}=0.04$ ) and there was no association with hematoma expansion (OR, $1.5 ; 95 \% \mathrm{Cl}, 0.3$ to $6.7, \mathrm{p}=0.58$ ).

\section{Conclusions}

In a single-center ICH registry, patients with ICH from vascular lesions had smaller hematoma volumes than patients with primary $\mathrm{ICH}$. 


\section{Poster 188}

\section{Expression of the Dual Endothelin-1/Signal Peptide[VEGF] Receptor (DEspR) on circulating leukocytes following Intracerebral Hemorrhage}

Riana L. Schleicher, Karen Li, Ravi Mylvaganam, Victoria L. M. Herrera, Nelson Ruiz-Opazo, Matthew B. Bevers, Joshua N. Goldstein, W. Taylor Kimberly

Massachusetts General Hospital Department of Neurology, Boston, MA, United States

\section{Background \& Purpose}

Neuroinflammation plays a critical role in secondary brain injury. Inhibition of the dual endothelin1/VEGFsp receptor (DEspR) protein on inflammatory cells improves survival in a hypertensive rodent model of spontaneous intracerebral hemorrhage $(\mathrm{ICH})$, and is a potential therapeutic target under development. We conducted a pilot observational study in human $\mathrm{ICH}$ patients to assess the expression of DEspR on peripheral leukocytes.

\section{Methods}

We performed a single center, prospective observational study of patients with non-traumatic ICH. Flow cytometry was performed on whole blood to detect and quantify DEspR surface expression on leukocyte subpopulations. Associations between DEspR expression and clinical and demographic data were evaluated using a Wilcoxon Rank-Sum test at $\alpha<0.05$.

\section{Results}

A total of 19 patients were enrolled. Mean age was $64 \pm 14$ years, $53 \%$ were female, median ICH volume was $39.1 \mathrm{~cm} 3$ [IQR 19.1,72.9], and median ICH score was 2 [IQR 2,3]. 74\% of patients survived to hospital discharge, and the median modified Rankin score (mRS) at 90 days was 4 [IQR 3,6]. Blood samples were drawn at $4.1 \pm 2.2$ days after symptom onset. DEspR expression on $>1 \%$ of cells was detected on CD11b+ neutrophils in 12 of 19 patients (63\%) and on CD11b+ monocytes (18 of 19 patients; $95 \%$ ), but not on lymphocytes, nor any leukocyte populations in a sample of healthy volunteers $(n=5)$. Among ICH patients, those with a history of hypertension $(n=13 ; 68 \%)$ had a higher level of DEspR expression on neutrophils and monocytes (sum: 16.6\%, IQR 9.5\%-34.0\%) versus those without hypertension (3.5\%, IQR 1.8\%-4.2\%, p=0.018).

\section{Conclusions}

DEspR is detected on the surface of neutrophils and monocytes in humans after ICH, and particularly those with a history of hypertension. Future additional work will ascertain the role of DEspR expression as a potential biomarker for outcome and inform further therapeutic development targeting this pathway. 
Poster 189

ICH outcomes and Reversal of Moderately Elevated INR 1.5 - 1.9

Aaron LacKamp, Jacob Reeder, Chaeli Stenuf, Adrien Caye, Justin Daniels, Kathrin Husmann

University of Kansas, Kansas City, KS, United States

\section{Background \& Purpose}

For ICH the Joint Commission CSTK-04 stroke measure encourages anticoagulant reversal when initial INR $>$ 1.4. Other guidelines are less definitive: AHA guidelines suggest reversing INR elevated due to VKA antagonist, and NCS/SCCM guidelines allow discretion regarding reversal for INR $<2$, but strongly encourages reversal of VKA-associated INR $>1.4$. While evidence supports reversal for INR $\geq 2.0$, little has been published on patients with INR 1.5-1.9. Given the lack of data, it is unclear whether this cohort of patients experiences benefit or harm from anticoagulant reversal.

\section{Methods}

We conducted a retrospective review of patients admitted to our institution from January 2015 through March 2021 to determine the rate of reversal and outcome for ICH with INR between 1.5-1.9. This date range was selected due to the completeness of the data.

\section{Results}

Thirty-nine patients presented with ICH and an INR of 1.5-1.9. These patients represented $5 \%$ of the 756 total ICH patients. Nineteen patients received reversal for mild elevation in INR, while twenty patients did not. The patients who received reversal were older (70.1 yrs vs $60.3 \mathrm{yrs}$ ), had higher INR (1.7 vs 1.6), and had higher ICH score (1.83 vs 1.4 ). For patients receiving reversal INR $<1.5$ was achieved in $70 \%$. Without reversal the trend was toward greater inpatient mortality ( $30 \%$ vs $21 \%$ ), and greater composite mortality-discharge-to-hospice (45\% vs 37\%). For low ICH scores there was a trend toward improved discharge MRS.

\section{Conclusions}

The use of reversal successfully lowered INR in patients with elevations 1.5-1.9. This reversal was without thrombotic complications. The decision to reverse was likely influenced by patient characteristics such as ICH score. Receiving reversal lessened the correlation between ICH score and discharge mRS. Further studies are warranted to determine causality. 


\title{
Poster 190
}

\section{ICH Score may Overestimate 30d Mortality in Patients Undergoing Minimally Invasive Evacuation. A Retrospective, Single-Center Study}

\author{
Bradley Douglas, Ryan Honomichl, Mark Bain, Alex Witek, Joao Gomes
}

Cleveland Clinic, Neurological Intensive Care Unit, Cleveland, OH, United States

\section{Background \& Purpose}

Minimally invasive techniques of surgical hematoma evacuation (MIS) have been developed. This investigation set out to assess the predictive performance of the ICH score on 30-day mortality in a cohort of patients who underwent MIS, compared to historical controls.

\section{Methods}

Single center, retrospective cohort study of adult patients admitted with diagnosis of primary ICH who underwent minimally invasive hematoma evacuation using a parafascicular approach with the BrainPath system (Nico Corporation).30-day mortality was compared between present sample and sample of Hemphill et al. overall and by ICH score subgroups with binomial tests. We further investigated whether age, ICH volume, or location differed between patients in the present sample who died vs. those that lived via Wilcoxon rank sum and Fisher's Exact Tests.

\section{Results}

Mean age $58+15.2$ years; $56.5 \%$ of the sample was male. Median ICH volume $44.1 \mathrm{cc}(35.7-59.2)$ and overall 30 -day mortality was $13.04 \%$. This was lower than overall mortality reported in Hemphill et al. $(45 \%, p<.05$.$) . Next, patients were categorized into two subgroups: patients with ICH score between 0$ and $2(N=29)$ and those with scores 3 or $4(N=17)$. No deaths occurred in patients with ICH score between 0 and 2 . In patients with scores 3 or 4 , mortality was $35 \%$. This was significantly different from a similar subgroup from Hemphill et al. $(72 \%, p<.05)$. Finally, patients who died in the present surgical sample were significantly older $(70.83+10.98$ vs $52.64+14.42$ years, $p<.05)$, however no differences were found in $\mathrm{ICH}$ volume or location.

\section{Conclusions}

The ICH score may overestimate 30-day mortality in patients undergoing MIS evacuation. Further research may lead to development and validation of a new grading scale for MIS patients that might have greater predictive value for mortality and/or functional outcome. 


\title{
Poster 191
}

\section{Intraventricular Thrombolytic Use and Reduction of Parenchymal Hematoma Volume in the CLEAR III Trial}

\author{
Santosh Murthy, Jens Witsch, David Roh, Radhika Avadhani, Alexander E. Merkler, Hooman Kamel, \\ Issam Awad, Daniel Hanley, Wendy C. Ziai \\ Department of Neurology, Weill Cornell Medicine, New York, NY, United States
}

\begin{abstract}
Background \& Purpose
Intraventricular thrombolysis reduces intraventricular hemorrhage (IVH) volume in patients with intracerebral hemorrhage (ICH), but it is unclear if a similar relationship exists with parenchymal ICH volume. We evaluated the association between intraventricular alteplase administration and parenchymal ICH volume, and functional outcomes.
\end{abstract}

\section{Methods}

We performed a post-hoc analysis of the Clot Lysis: Evaluating Accelerated Resolution of Intraventricular Hemorrhage (CLEAR III) trial, where patients with ICH $(<30 \mathrm{~mL})$ and large obstructive IVH were randomized to receive periodic administration of either intraventricular alteplase or normal saline. The primary outcome was a change in hematoma volume between stability and end-of-treatment computed tomography scans. Secondary outcomes were poor outcome (modified Rankin score $>3$ ) and mortality, assessed at 6 months. We assessed the relationship between alteplase and change in ICH volumes, and the association between change in $\mathrm{ICH}$ volume and 6-month outcomes, using multiple linear and logistic regression models, respectively.

\section{Results}

Of 454 enrolled patients with ICH, 230 (51\%) received intraventricular alteplase. The alteplase group had a greater mean reduction in ICH volume compared to the placebo group $(1.8 \mathrm{~mL}$ versus $0.4 \mathrm{~mL}$, $\mathrm{p}<0.001$ ). Multiple linear regression adjusted for demographics, admission ICH and IVH volumes, ICH location, and antithrombotic therapy, showed a significant association between alteplase administration and decrease in parenchymal ICH volume (beta $0.51,95 \% \mathrm{Cl}, 0.36-0.77, \mathrm{p}<0.001$ ). Secondary analysis did not show an association between decrease in $\mathrm{ICH}$ volume and poor outcome (aOR, 1.2; $95 \% \mathrm{Cl}, 0.9$ $1.5)$, or with mortality (aOR, $1.1 ; 95 \% \mathrm{Cl}, 0.8-1.4)$, likely due to the burden of remaining IVH.

\section{Conclusions}

In this secondary analysis of the CLEAR III trial, intraventricular alteplase was associated with a small reduction in parenchymal ICH volume, highlighting a possible communication between the ventricular cavity and brain parenchyma. These results highlight exploring this novel treatment for decreasing $\mathrm{ICH}$ volume and potentially improving outcomes. 


\section{Poster 192}

\section{Longitudinal Associations Between Neuroinflammatory Biomarkers and Edema Volumes in Patients with Acute Supratentorial Intracerebral Hemorrhage}

Jay B. Lusk, Jesse Troy, Nathaniel Nowacki, Peter G. Kranz, Christopher D. Lascola, Maureen Maughan, Michael L. James, Daniel T. Laskowitz

Duke University School of Medicine, Department of Neurology, Durham, NC, United States

\section{Background \& Purpose}

Progress in improving ICH morbidity and mortality has been limited by a lack of clear biomarkers of disease severity, which could be used to guide the development of novel therapies. In particular, it is increasingly clear that neuroinflammation after ICH may be a key factor in ICH outcomes. We therefore developed and assessed a panel of ten biomarkers, measured on several standardized time-points after $\mathrm{ICH}$, and determined their associations with edema volume.

\section{Methods}

We identified a group of ten immunological biomarkers that could be easily commercially obtained as a panel and had theoretical relevance to neuroinflammation. We initially evaluated these biomarkers in blood samples obtained from randomly selected patients with ICH who presented to Duke University hospital. We then longitudinally evaluated the relationship between these biomarkers and edema volume over five days after $\mathrm{ICH}$, as measured on both $\mathrm{CT}$ and $\mathrm{MRI}$, using mixed-effects linear regression in a convenience sample of patients treated with $\mathrm{CN}-105$, an investigational drug for neuroprotection in $\mathrm{ICH}$.

\section{Results}

Higher C-reactive protein levels were associated with greater edema volumes. Interleukin (IL)-1 receptor antagonist, IL-8, IL-10, and monocyte chemotactic protein levels were high at day 0 , and decreasing levels from days 1-5 were associated with greater edema volumes. IL1-B, IL-23, and von Willebrand factor levels were directly associated with edema volume from days $0-3$, but were inversely associated with edema volume from days 3-5. Matrix Metalloproteinase-9 and IL-17 levels were high at day 0, and from days 1-3 were associated with greater edema volumes, while from days 3-5 were inversely associated with edema volumes.

\section{Conclusions}

We observed several distinct categories of associations between inflammatory biomarker levels and edema volumes in $\mathrm{ICH}$, most of which have not been documented in human $\mathrm{ICH}$. Future research is needed to characterize the prognostic and therapeutic implications of these findings. 


\section{Poster 193}

\section{Mediation Effects of Mean Hounsfield Unit on Relationship between Hemoglobin and Expansion of Intracerebral Hemorrhage}

Yong Soo Kim, Han-Gil Jeong, Hee-Yun Chae, Beom Joon Kim, Jihoon Kang, Jun Yup Kim, Tackeun Kim,, Jae Seung Bang, Hee-Joon Bae, Chang Wan Oh, Moon-Ku Han

Department of Neurology, Seoul National University Bundang Hospital, Seongnam-si, Republic of Korea

\section{Background \& Purpose}

Low hemoglobin levels are known to be associated with hematoma expansion (HE) and poor functional outcome in patients with intracerebral hemorrhage $(\mathrm{ICH})$. However, it is not yet known whether low hemoglobin itself causes HE directly or is merely a confounder. Thus, we investigated the mediation effect of the mean Hounsfield unit (HU) of hematoma, a potential marker of local hemostasis, on the relationship between low hemoglobin and expansion of intracerebral hemorrhage.

\section{Methods}

Overall, 232 consecutive patients with ICH who underwent non-contrast computed tomography (NCCT) within 12 hours since onset were included. The mean HU and hematoma volume on NCCT were investigated using semi-automated planimetry. HE was defined as an increase in hematoma volume $>33 \%$ or $6 \mathrm{~mL}$. The respective associations among the hemoglobin level, mean HU, and HE were analyzed using multivariable regression analysis. Mediation analysis was performed to examine the potential causal association among the three.

\section{Results}

HE occurred in $34.5 \%$ of patients; hemoglobin levels were inversely associated with HE occurrence (adjusted odds ratio, 0.89; $\mathrm{p}=0.02$ ). The mean $\mathrm{HU}$ of the hematoma was lower in patients with $\mathrm{HE}$ than in patients without $\mathrm{HE}(58.5 \pm 3.3$ vs. $56.8 \pm 3.0 ; p<0.01)$. Hemoglobin levels on admission were linearly related to the mean $\mathrm{HU}$ (adjusted $\beta, 0.35 ; \mathrm{p}<0.01$ ) after adjusting for known $\mathrm{HE}$ predictors (time from onset to $\mathrm{CT}$, antithrombotic use, hematoma volume). Causal mediation analysis showed a significant mediation effect of the mean $\mathrm{HU}$ on the association between hemoglobin levels and HE $(p<0.01)$. The proportion of indirect effect through the mean $\mathrm{HU}$ among the total effect was $37 \%(p=0.04)$.

\section{Conclusions}

The mean $\mathrm{HU}$ of the hematoma fully mediated the association between hemoglobin levels and $\mathrm{HE}$ occurrence. Therefore, the mean $\mathrm{HU}$ of the hematoma may be a robust marker of impaired hemostasis in patients with $\mathrm{ICH}$. 


\section{Poster 194}

\section{Mortality after Spontaneous Intracerebral Hemorrhage: Does Surgery Improve Outcome in a Matched Cohort}

Yasser B. Abulhasan, Jeanne Teitelbaum, Khalsa Al-Ramadhani, Mark R. Angle

Neurological Intensive Care Unit, McGill University, Montreal, Canada; Faculty of Medicine, Kuwait University, Kuwait City, Kuwait

\section{Background \& Purpose}

Spontaneous intracerebral hemorrhage (ICH) is a devastating neurological disease with a historical 30day mortality of $40 \%$. As treatment and critical care management after ICH advanced over the past decade, we sought to determine modern-day causes and predictors of mortality, and evaluate the effect of surgery performed as a life-saving measure after supratentorial ICH on patient-related outcomes.

\section{Methods}

A retrospective cohort study of 361 patients with ICH admitted between 2010 and 2017 to a neurological critical care unit was performed. The primary outcome was 30-day in-hospital mortality, which permitted uniform analysis of cause-specific mortality. Secondary outcomes were unfavorable functional outcome (modified Rankin Score 4-6), and all-cause 90-day mortality. Using propensity score matching, we studied the association between surgery and 30- and 90-day mortality, and unfavorable functional outcome in patients with supratentorial ICH.

\section{Results}

The 30 -day mortality rate after ICH was $25 \%$ (89/361). The most common adjudicated primary causes of mortality were direct effect or progression of the primary hemorrhage (54\%), refractory cerebral edema (21\%), and medical complications (11\%). Independent admission predictors significantly associated with 30-day mortality were older age, ICH volume $\geq 30 \mathrm{~mL}$, hydrocephalus, lower Glasgow Coma Scale (GCS) score, lower hemoglobin level, lower platelet count, and mechanical ventilation. Patients with supratentorial ICH who had surgeries had longer hospital (p-value: 0.001 ) and ICU (p-value: <0.001) length of stay compared to supratentorial ICH patients without surgeries. In matched analyses, lifesaving surgery for 37 patients with supratentorial ICH (median GCS was 7 [interquartile range 6-10]) reduced the odds of 30 -day mortality by $73 \%$, however did not significantly reduce the odds of 90 -day mortality and unfavorable functional outcome.

\section{Conclusions}

The direct effect or progression of the primary hemorrhage remains the most common cause for 30-day mortality after $\mathrm{ICH}$. Regarding surgery for neurologically deteriorating/deteriorated patients with supratentorial ICH, although feasible, it only reduces 30-day mortality. 


\title{
Poster 195
}

\section{Outcomes Associated with High-Dose Versus Low-Dose 4-factor Prothrombin Complex Concentrate in Factor Xa Inhibitor-Associated Intracranial Hemorrhage}

\author{
Spencer Davis, Stephanie Chauv, Abby Hickman, Sara Kjerengtroen, Dave Collingridge, Gabriel V. \\ Fontaine
}

Intermountain Healthcare, Department of Pharmacy, Salt Lake City, UT, United States

\begin{abstract}
Background \& Purpose
4-factor prothrombin complex concentrate (4FPCC) is used off-label for factor Xa inhibitor (FXal) associated intracranial hemorrhage (ICH). Guideline recommendations provide various 4FPCC dosing regimens for FXal reversal in this setting. We evaluated 4FPCC weight-based dosing and outcomes in FXal-associated ICH.
\end{abstract}

\section{Methods}

We conducted a multi-center, retrospective, cohort study of ICH patients between July 2017 and February 2020. Patients were greater than 18 years of age, received 4FPCC, and were taking apixaban, rivaroxaban, or edoxaban. Exclusion criteria were receipt of $4 \mathrm{FPCC}$ for non-ICH related causes, no repeat brain computed tomography (CT) within 12 hours, or death within 24 hours. Patients were separated into high- (>=35 units $/ \mathrm{kg}$ ) or low-dose (<35 units $/ \mathrm{kg}$ ) 4FPCC groups. The primary outcome was hemostasis achievement, defined as radiologist documentation on repeat brain CT indicating no or minimal expansion of ICH. Secondary outcomes included in-hospital mortality, ICU length of stay (LOS), hospital LOS, discharge disposition, and thrombotic events. Outcomes were evaluated with multivariable logistic regression.

\section{Results}

Of 390 patients, 89 were included with 74 and 15 in the high- vs low-dose groups, respectively. Mean (SD) age was $76.6( \pm 10.8)$ years. Most were taking FXal for atrial fibrillation $(76.4 \%)$ and apixaban was the most common FXal (65.2\%). Mean 4FPCC dose was 3,662 units (44.2 units/kg) in the total population, 3,910 units ( 47.9 units $/ \mathrm{kg}$ ) in the high-dose group, and 2,438 units $(25.8$ units $/ \mathrm{kg}$ ) in the lowdose group. Hemostasis achievement was greater in the high- vs low-dose group (89.2\% vs 46.7\%; OR 11.2; $95 \% \mathrm{Cl} 2.4-52.6, \mathrm{P}=0.002$ ). Thrombotic events were $8.2 \%$ and $6.7 \%$ in the high vs low-dose groups, respectively (OR $0.8 ; 95 \% \mathrm{Cl} 0.08-8.2, \mathrm{P}=0.87$ ). No statistically significant differences were found in secondary outcomes.

\section{Conclusions}

In patients with FXal-associated ICH, high-dose 4FPCC was associated with increased odds of hemostasis achievement. There was no difference in thrombotic events. 


\title{
Poster 196
}

\section{Pilot Observational Study in ICH Patients Detects Increased 'Rogue' Neutrophil Subset Implicated in Neutrophil-mediated Secondary Tissue Injury: A Potential Actionable Target}

Courtney Takahashi, David M. Greer, Mai Q. Nguyen, Julie Z. Mosaddeghi, Ridiane Denis, Victoria L.M. Herrera, Nelson Ruiz-Opazo

Boston University, Department of Neurology, Boston, MA, United States

\begin{abstract}
Background \& Purpose
Dysregulated "rogue" neutrophils drive neutrophil-mediated secondary tissue injury leading to cerebral edema and neuronal toxicity in $\mathrm{ICH}$, similar to multi-organ failure in ARDS. Increased neutrophil lymphocyte ratio (NLR) associated with poor outcomes in ICH and ARDS supports this notion, but no actionable target has been found. Identifying a targetable "rogue" neutrophil subset that correlates with NLR and ICH-severity parameters is critical to identifying novel therapies for ICH.
\end{abstract}

\section{Methods}

Using flow cytometry analysis, we studied whether increased "rogue" neutrophils, defined by coexpression of the dual endothelin1/signal peptide receptor (DEspR) and neutrophil-activation marker $\mathrm{CD} 11 \mathrm{~b}$, are elevated in a pilot observational study in $\mathrm{ICH},(\mathrm{n}=12)$. We used a neutrophil-optimized flow cytometry protocol: analysis of fresh EDTA-anticoagulated whole blood (<1-hour from sampling), average 2.6-days from admission, no CD45/CD14 antibody-staining as their binding changes neutrophils, duplicates to ascertain results, and matched-fluorophore isotypes as negative-controls. We performed Spearman Rank Correlation analysis of the number(\#) of DEspR+CD11b+ "rogue" neutrophils (K/ $\mu \mathrm{L})$ with $\mathrm{NLR}, \mathrm{ICH}$-score, GCS-score, 90d-mRS, and perihematomal edema (PHE) volumes. We define significant $(p<0.05)$ high correlation (correlation coefficient rho $>0.5$ ) $\neg$ with power 0.8 as robust-correlation; significant high correlation with power 0.7 as correlation-trends.

\section{Results}

Pilot observations detect increased \#DEspR+CD11b+ "rogue" neutrophils in ICH patients with robustcorrelation with: a] 90d mRS: rho=0.849), b] PHE-volume rho=0.776, c] GCS-score rho [-0.765], and correlation-trends with d] NLR rho $=0.727$, and e] ICH-score rho $=0.721$. NLR exhibited robust-correlation with a] PHE-volume only rho=0.755; correlation-trends with b] GCS-score rho=[-0.725] and c] ICH-score rho $=0.693$, but no correlation with d] $90 \mathrm{~d} \mathrm{mRS}$. Comparative analysis of $\mathrm{ICH}$ survivors vs non-survivors showed significant association of \#DEspR+ "rogue" neutrophils with mortality $(p=0.0125)$ but not with NLR.

\section{Conclusions}

Pilot observations support the hypothesis that DEspR+ "rogue" neutrophils comprise a potentially actionable therapeutic target to reduce neutrophil-mediated PHE and secondary brain-injury in ICH, providing basis for further study. 


\title{
Poster 197
}

\section{Rapid blood pressure reduction in the first 6 hours after intracerebral hemorrhage predicts acute kidney injury (AKI)}

\author{
Vishank Arun Shah, Shilpa Haldal, Ebonye Green, Krishna Nalleballe, Kelly-Ann Patrice, Mhd Ezzat \\ Zaghlouleh
}

University of Arkansas for Medical Sciences, Department of Neurology, Little Rock, AR, United States

\section{Background \& Purpose}

Acute kidney injury (AKI) is common and associated with worse outcomes in patients with intracerebral hemorrhage $(\mathrm{ICH})$. We aimed to identify predictors of AKI in ICH patients and explore the relationship between blood pressure reduction and AKI.

\section{Methods}

We conducted a retrospective analysis on a cohort of spontaneous $\mathrm{ICH}$ patients admitted to a tertiary hospital in Arkansas. Demographics, comorbidities, in-hospital complications, baseline creatinine and blood pressure (SBP/MAP) recordings in the first 72 hours of hospitalization were extracted. Univariate analysis assessed factors associated with development AKI during hospitalization. Subsequently, multivariable logistic regression models predicting new AKI were generated.

\section{Results}

Of 524 patients included in the analysis, new AKI occurred in 94 (17.9\%). On univariate analysis, factors associated with AKI included Black race $(43.6 \%$ v. 30.7\%, $p=0.016)$, history of chronic kidney disease (19.1\% v. 11.2\%, p=0.035), baseline creatinine (2.1 [2.2] v. 1.3 [1.7] mg/dl, $p=0.003$ ), acute myocardial infarction (MI) (24.5\% v. 10.2\%, p<0.001). While the admission MAP and SBP were not associated with outcome, mean 6-hour MAP (87.6 [16.2] v. 83.3 [14.5] mm Hg) and SBP (126.4 [22.4] v. 132.3 [22.7] mm $\mathrm{Hg}$ ) recordings were associated with AKI. Lowest mean MAP (60.5 [13.9] v. 55.5 [14.8], p=0.004) and SBP (132.3 [22.7] v. 126.4 [22.4], $p<0.001$ ) recordings in the first 72 hours were also associated with AKI. On multivariable logistic regression, best performing model predicting AKI included baseline creatinine (OR: 1.18 [1.05-1.33] per mg/dl), MI (OR: 2.57 [1.29=5.14]), and SBP reduction of $>80 \mathrm{~mm} \mathrm{Hg}$ in first 6 hours (OR: 4.53 [1.44-14.2]) (ROC area: 0.787 [0.73-0.85]). Other models for AKI included creatinine, MI and either 6 hour SBP reading < 120 mm Hg (OR: 2.5 [1.26-4.99]) (ROC Area: 0.731 [0.664-0.798]) or 6 hour MAP reading < $80 \mathrm{~mm} \mathrm{Hg}$ (OR: 1.18 [1.05-3.13]) (ROC area: 0.731 [0.67-0.795]). Age, CKD, black race and pneumonia did not independently predict development of AKI in the models.

\section{Conclusions}

Rapid blood pressure reduction in the first 6 hours to normotensive blood pressure goals predicts development of acute kidney injury after ICH. 


\title{
Poster 198
}

\section{Soluble ST2 as a Marker for Acute Neurovascular Injury: A Comprehensive Survey of WHO Neurological Disorders}

\author{
Caroline Booraem, Karen Li, W. Taylor Kimberly, Matthew B. Bevers
}

Division of Neurocritical Care, Brigham and Women's Hospital, Boston, MA, United States

\section{Background \& Purpose}

We have previously demonstrated elevated plasma levels of soluble ST2 (sST2) in acute ischemic stroke (AIS) and subarachnoid hemorrhage (SAH), but this marker has not been investigated in other neurological diseases. The purpose of this study was to survey the ten World Health Organization (WHO) categories of neurological diseases to ascertain which types of neurological disease are associated with elevated SST2.

\section{Methods}

The Mass General Brigham Health biobank was queried to identify patients with each disease of interest and a plasma sample obtained after diagnosis. A total of 361 samples were obtained across disease categories, and SST2 concentrations were measured using a SST2 ELISA. The ten disease categories were dementia, infection, pain disorders, epilepsy, headache, multiple sclerosis, Parkinson's disease, nutritional disorders, stroke and traumatic brain injury (TBI). Measured concentrations were compared between each disease category and existing prospectively collected cohorts of patients with AIS, SAH, intraparenchymal hemorrhage (IPH) and TBI. As a quality control measure, linear regressions were done to examine the effect of patient age at collection and duration of sample storage.

\section{Results}

The level of sST2 in stroke (median $63.4 \mathrm{ng} / \mathrm{mL}$, [IQR 34.4, 118.2]) and TBI (54.6 ng/mL, [51.4, 93.3]) were significantly higher compared to the other disease categories $(27.7 \mathrm{ng} / \mathrm{mL},[22.9,34.4], p<0.002$ for each pairwise comparisons). This difference was also evident for each stroke subtype (AIS: $38.3 \mathrm{ng} / \mathrm{mL}$ [32.1, 60.6], SAH: $96.6 \mathrm{ng} / \mathrm{mL}[58.6,151.6], \mathrm{IPH}: 62.9 \mathrm{ng} / \mathrm{mL}[30.6,115.0], \mathrm{p}<0.0001$ for each pairwise comparison). There was no association between patient age $(\beta=-0.00038,95 \% \mathrm{Cl}[-0.0027,0.0020]$, $p=0.756)$ or sample storage duration $(\beta=-0.0055,95 \% \mathrm{Cl}[-0.028,0.017], p=0.623)$ and sST2 level.

\section{Conclusions}

These results suggest that SST2 is a marker specific to acute neurovascular injury that is not seen in general neurological disease. These findings warrant further research into the mechanism associating sST2 with stroke and its effect on severity and outcome. 
Poster 199

\section{Thalamic Involvement In Intracerebral Hemorrhage As A Predictor of Tracheostomy}

Charlotte Zhong, Christopher D. Nguyen, Connie Chung, Li Ding, Peggy Nguyen, May Kim-Tenser, Benjamin Emanuel, Roy A. Poblete, Sebina Bulic

University of Southern California, Keck School of Medicine Department of Neurology, Los Angeles, CA, United States

\section{Background \& Purpose}

Many patients with intracerebral hemorrhage $(\mathrm{ICH})$ are often intubated for airway protection due to decreased level of consciousness. Components of the ICH score have been found to potentially predict the need for tracheostomy, but limited data exists on the role of $\mathrm{ICH}$ location. We hypothesize that thalamic involvement in $\mathrm{ICH}$ increases the risk of developing chronic respiratory failure requiring tracheostomy.

\section{Methods}

We performed a retrospective review of adult patients with ICH from a high-volume academic stroke program from 2014-2019 and identified patients with primary supratentorial ICH who were intubated during hospitalization. The primary outcome was tracheostomy placement. We assessed the effect of thalamic versus non-thalamic insult from ICH in requiring tracheostomy via multivariate analysis. We also looked at the effect of hemorrhage location on extubation failure, defined as needing reintubation within 72 hours of extubation, as a secondary outcome.

\section{Results}

A total of 84 patients with ICH met study criteria and were included in the analysis, 38 of which required tracheostomy. Development of chronic respiratory failure requiring a tracheostomy occurred in 21 of 33 patients (63.6\%) that had $\mathrm{ICH}$ with thalamic involvement compared to 17 of 51 patients (33.3\%) with non-thalamic ICH. Patients with thalamic bleeds were 1.6 times more likely to require tracheostomy than patients with non-thalamic bleeds $(p=0.04)$. Additionally, secondary outcomes included extubation failure, which occurred in 13 patients, 6 of which had thalamic bleeds and 7 had non-thalamic bleeds. Patients with thalamic involvement were twice as likely to experience extubation failure, although this did not reach significance $(p=0.27)$.

\section{Conclusions}

Patients with thalamic ICH were more likely to require tracheostomy compared to those without thalamic involvement. Overall, these patients may benefit from early tracheostomy to reduce duration of mechanical ventilation, ICU length of stay, and hospitalization costs. 


\title{
Poster 200
}

\section{The ICH and FUNC Scores in Non-white Patients}

\author{
Nirupama Yechoor, Jessica R. Abramson, Juan Pablo Castello, Evangelos Pavlos Myserlis, Haitham Samir \\ Alabsi, Sophia Keins, Christina Kourkoulis, Alessandro Biffi, Jonathan Rosand \\ Department of Neurology, Massachusetts General Hospital, Boston, MA, United States
}

\section{Background \& Purpose}

The ICH and FUNC scores are widely used prognostic indicators after intracerebral hemorrhage (ICH). However, racial disparities after ICH have been described, including increased rates of recurrent stroke and greater cognitive impairment in non-white patients. This study aims to investigate if the ICH and FUNC scores correlate with functional outcomes for racial minorities given omission of social determinants of health.

\section{Methods}

A retrospective review was conducted of the prospectively ascertained Massachusetts General Hospital ICH Cohort. Demographic data, ICH location and volume, admission GCS, and pre-admission history of cognitive impairment were used to calculate clinical scores. Student's t-test and chi-square tests were used to compare sociodemographic data. Linear regression was used to compare the relationship between scores and discharge modified-Rankin Scores (mRS).

\section{Results}

Of 3,186 patients, non-white patients comprise $15.8 \%$ of the population, are younger (median age of 67 compared to 75 in white patients), and were more likely to have diagnosed hypertension ( $88 \%$ compared to $78 \%$ in white patients). Significant differences in social determinants of health include lower median incomes, higher rates of baseline disability/unemployment, and lower education (Table 1). Admission ICH and FUNC scores were available for 2,043 and 2,430 patients respectively (Table 2), with their distributions shown in Figure 2. The ICH score had a significant positive correlation with discharge $\mathrm{mRS}$, and the FUNC score had a significant negative correlation to discharge mRS for white and non-white patients (Figure 3).

\section{Conclusions}

The ICH and FUNC scores aid clinicians and families in important medical decision-making. However, it was unknown if these scores apply to diverse populations. Our findings show that both scores correlate to discharge mRS after ICH, suggesting they are appropriate clinical tools regardless of race. Future studies should incorporate longer-term functional outcome markers to investigate if any correlation exists after discharge, where social determinants of health may exert greater impact. 
Poster 201

\section{Using Non-Hemorrhagic Imaging Markers to Determine Cerebral Small Vessel Disease Etiology in Patients with Isolated Lobar Intracerebral Hemorrhage}

Alvin S. Das, Elif Gökçal, Robert W. Regenhardt, Mitchell J. Horn, Kristin M. Schwab, Nader Daoud, Anand Viswanathan, William T. Kimberly, Joshua N. Goldstein, Alessandro Biffi, Natalia Rost, Jonathan Rosand, Lee H. Schwamm, Steven M. Greenberg, M. Edip Gurol

Department of Neurology, Massachusetts General Hospital, Harvard Medical School, Boston, MA, United States

\section{Background \& Purpose}

Isolated lobar intracerebral hemorrhage (ICH) can either be due to cerebral amyloid angiopathy (CAA) or hypertensive cerebral small vessel disease (HTN-CSVD). Herein, we aimed to understand the ability of non-hemorrhagic imaging markers (NHIM) (lacunes, white matter hyperintensity patterns [WMHP], perivascular spaces [PVS]) to differentiate CAA from HTN-CSVD in this patient cohort.

\section{Methods}

Consecutive patients with isolated lobar ICH were categorized into four cSVD groups based on the presence/location of NHIM: CAA pattern, HTN-cSVD pattern, mixed NHIM, and no NHIM. CAA pattern was defined as the presence of any number of CAA features: lobar lacunes, severe centrum semiovale PVS, or multiple subcortical spots WMHP. HTN-cSVD pattern included any number of HTN-cSVD features: deep lacunes, severe basal ganglia PVS, or peri-basal ganglia WMHP. Mixed NHIM included at least one imaging finding from either pattern. We hypothesized that HTN-cSVD pattern and mixed NHIM patients were more likely to have left ventricular hypertrophy (LVH), a marker for HTN-cSVD. A logistic regression model consisting of age, sex, vascular risk factors, and LVH was constructed to determine associations with HTN-cSVD pattern.

\section{Results}

261 (15\%) out of $1791 \mathrm{ICH}$ patients had isolated lobar ICH. LVH was more common in HTN-cSVD pattern vs. CAA pattern $(50 \%$ vs. $20 \%, p<0.001)$, but similar between HTN-cSVD pattern and mixed NHIM $(50 \%$ vs. $39 \%, p=.418$ ). LVH was more common in mixed NHIM compared to no NHIM ( $39 \%$ vs. $12 \%, p=$ 0.005). There was a trend toward higher serum creatinine levels in HTN-cSVD pattern (1.00 \pm 0.32 $\mathrm{mg} / \mathrm{dL}$ ) compared to CAA pattern $(0.96 \pm 0.25 \mathrm{mg} / \mathrm{dL}, \mathrm{p}=0.072)$. Age and LVH were independent predictors of HTN-cSVD pattern ( $p<0.001$ for both comparisons) despite controlling for sex and vascular risk factors.

\section{Conclusions}

NHIM can aid in CSVD determination in patients with isolated lobar ICH. 


\title{
Poster 202
}

\section{When is the Transfer of Intracranial Hemorrhage Futile?: Insights from Withdrawal of Life-Sustaining Support Soon After Transfer to a Tertiary Referral Center}

\author{
Monica Ann Krause, Eelco Wijdicks, Sara Hocker \\ Department of Neurology, Mayo Clinic Rochester, Rochester, MN, United States
}

\section{Background \& Purpose}

Intracranial hemorrhages (ICH) account for $10-15 \%$ of strokes in the United States. Despite transfer to tertiary centers and advanced practices, patients may succumb to their hemorrhage or families may pursue comfort-based care shortly after transfer. This presents emotional, financial, and resource costs to patients, families, and medical institutions. We sought to determine predictors of early transition (within 24 hours of presentation) to comfort-based care or death in ICH by analyzing the presenting characteristics of ICH patients transferred to our Neurologic Intensive Care Unit (NICU).

\section{Methods}

We performed a retrospective review of consecutive patients with non-traumatic ICH transferred to the NICU at Mayo Clinic Hospital, St. Marys campus, in Rochester, MN from 2014-2019. Demographics collected included code status, anticoagulant use, systolic blood pressure, hematoma characteristics, level of consciousness (based on GCS and Full Outline of Unresponsiveness [FOUR] score), and interventions offered/completed in the NICU. Analysis with Kruskal Wallis or Pearson's chi squared tests was completed. Data was modeled with univariate and multivariate logistical regression.

\section{Results}

Preliminary results (107 of 318 patients) demonstrated older age, increased medical comorbidities, and lower presenting level of consciousness in early transition to comfort-based care or death. Univariate modeling indicated increased likelihood for anticoagulant use (OR 3.17, [CI 1.05-9.54]), midline shift (OR 7.80[Cl 2.30-26.47]), hydrocephalus (OR 9.05, [Cl 2.83-28.91]), hematoma expansion (OR 8.00, [Cl 1.5940.20]), ICH score (OR 19.08, [Cl 3.01-121.02]), and admission from skilled nursing facility (OR 10.20, [CI 2.63-39.57]). Multivariate modeling revealed predictive value of code status (OR 9.61,[Cl 1.92-48.12]) and ICH score (OR 33.58,[Cl 3.00-376.23]).

\section{Conclusions}

Our study preliminarily suggests presenting characteristics may predict transition to comfort-based care or death within 24 hours of transfer to a tertiary center. These predictive characteristics may provide further guidance when counseling families and patients prior to transfer to reduce financial, emotional, and resource costs to patients, families, and medical communities. 
Poster 203

\title{
Blood Pressure Variability Indices for Outcome Prediction after Thrombectomy in Large Vessel Occlusion Stroke Using High Resolution Blood Pressure Data
}

\author{
Corinne Inauen, Jens M. Boss, Mira Katan, Christian Strässle, Stefan Y. Bögli, Emanuela Keller
}

Neurocritical Care Unit, Dept. of Neurosurgery and Inst. of Intensive Care Medicine, University Hospital of Zurich, Switzerland

\section{Background \& Purpose}

Blood pressure variability (BPV) has been associated with outcome after endovascular thrombectomy (EVT) in acute large vessel occlusion (LVO) stroke using various indices and measurement frequencies. We aimed to provide the optimal sampling frequency and BPV index for outcome prediction using high resolution blood pressure (BP) data.

\section{Methods}

Patient characteristics, three-month outcome, and intra-arterial BP measured at $1 \mathrm{~Hz}$ for up to 24 hours were extracted retrospectively from 34 patients admitted to the neurocritical care unit between 2017 and 2020 after EVT. Outcome was dichotomized (mRS 0-2 favorable/3-6 unfavorable) and associated with systolic BPV (as calculated using standard deviation, coefficient of variation, averaged real variability, successive variation (SV), number of trend changes, and a spectral approach using the power of specific BP frequencies) using the Mann-Whitney $U$ test.

\section{Results}

Among 34 patients (age 72 \pm 12.7$) 10$ (29.4\%) and 24 (70.6\%) achieved a favorable and unfavorable functional outcome at three months, respectively. No group differences were found in initial NIHSS (median $14[8,19], p=0.59)$, time between onset to groin puncture, symptomatic intracerebral hemorrhage or grade of recanalization ( $94.1 \%$ success rate). Mean absolute systolic BP $(131 \pm 18 \mathrm{mmHg}$, $\mathrm{p}=0.99$ ) did not differ between groups during the monitoring time. BPV only reached predictive significance using SV extracted from down sampled (average over 5 minutes) systolic BP data (median $4.8 \mathrm{mmHg}[3.8,7.1])$ vs. $7.1 \mathrm{mmHg}[5.5,9.7], \mathrm{p}=0.031, \mathrm{AUC}=0.74$ (Cl: 0.57-0.85)), or correspondingly the power of mid-range frequencies between $1 / 20$ to $1 / 5$ minutes $(p=0.020, A \cup C=0.75$ (Cl: $0.59-0.86)$ ).

\section{Conclusions}

The use of high resolution BP data together with downsampling proved essential to find relevant changes in systolic BP as noise can be adequately suppressed (confirmed by the significance of the power of mid-range frequencies). These results demonstrate how high-resolution data can be processed for effective outcome prediction. 


\section{Poster 204}

\section{Duration of Heightened Risk of Acute Ischemic Stroke after Acute Systolic Heart Failure Hospitalization}

Devin J. Burke, Tehniyat Baig, Parag Goyal, Cenai Zhang, Alexander E. Merkler

Division of Neurocritical Care, Weill Cornell Medicine, New York Presbyterian, New York, NY, United States

\section{Background \& Purpose}

Heart failure can increase the activity of procoagulant factors and risk of thromboembolic complications resulting in a 2- to 3-fold increased risk of ischemic stroke. Prior studies looking at the relationship between heart failure and stroke risk are mostly of relatively smaller sample sizes, do not adjust or stratify for atrial fibrillation, and do not distinguish between ischemic and hemorrhagic stroke risk. There remains equipoise in the degree to which heart failure increases the risk of ischemic stroke and the duration of elevated risk. Therefore, we aimed to assess the magnitude and duration of ischemic stroke risk in a large diverse sample of acute systolic heart failure patients with and without atrial fibrillation. We hypothesized that acute systolic heart failure will be independently associated with an increased risk of ischemic stroke that decreases over time.

\section{Methods}

We conducted retrospective cohort study using inpatient and outpatient claims data from a nationally representative $5 \%$ sample of Medicare beneficiaries. The study's exposure variable is a hospitalization for acute systolic heart failure as ascertained by ICD discharge diagnosis codes. Primary outcome was ischemic stroke as determined by discharge diagnosis code. Cox proportional hazards analysis was used to evaluate the association between acute systolic heart failure and ischemic stroke while adjusting for demographics, vascular risk factors, and co-morbidities. We stratified the cohort by patients with and without atrial fibrillation.

\section{Results}

After adjusting for demographics, stroke risk factors, and Charlson comorbidities, the risk of ischemic stroke after presentation with acute systolic heart failure was significantly elevated and maximal within the first 30 days after discharge. The risk of ischemic stroke within the first 30 days was higher in patients without comorbid atrial fibrillation (HR: 4.6; $95 \% \mathrm{Cl}, 4.0-5.3$ ) compared to patients with comorbid atrial fibrillation (HR: $2.4 ; 95 \% \mathrm{Cl}, 2.1-2.7$ ). . It was significantly elevated up to 330 days in patients without atrial fibrillation (HR: $2.1 ; 95 \% \mathrm{Cl}, 1.7-2.7$ ) compared to a significant elevation up to 60 days in patients with atrial fibrillation $(\mathrm{HR}, 1.4 ; 95 \% \mathrm{Cl}, 1.2-1.6)$.

\section{Conclusions}

Hospitalization for acute systolic heart failure is associated with an elevated risk of acute ischemic stroke which is maximal within 30 days post-hospitalization. The risk is higher and prolonged in patients without comorbid atrial fibrillation. 
Poster 205

\section{Effect of Navitoclax as the Senolytic Drug on Transient Focal Ischemia in Rats}

Tae Jung Kim, Young-Ju Kim, Soo-Hyun Park, Sang-Bae Ko

Department of Neurology and Critical Care Medicine, Seoul National University Hospital, Seoul, Republic of Korea

\section{Background \& Purpose}

Senolytic drugs are thought to target senescent cells and show anti-inflammatory effect in cancers and cardiovascular diseases. Navitoclax (ABT-263), which is a type of senolytic drug, selectively eliminates senescent cells. In this study, we investigated the effects of Navitoclax on focal ischemia in rat brains.

\section{Methods}

We induced focal ischemia in brain by transient intraluminal middle cerebral artery occlusion (MCAO) and after induction of MCAO, rats were randomly separated into two experimental groups: MCAO with administration of Navitoclax (10 mg/ $\mathrm{kg}$, Navitoclax group) via intravenous routes and MCAO with administration of phosphate-buffered saline (PBS group) In addition, sham-operated rats received the same surgery process without the MCAO. Functional recovery of rats was evaluated at 1, 3, 8, and 15 days following MCAO using the modified neurological severity score (mNSS). Moreover, the infarct volume was evaluated using serial sections collected for TTC staining at 4 days after MCAO and inflammatory markers were assessed.

\section{Results}

The infarct volume was reduced in the Navitoclax treated group compared with PBS group at 4 days after MCAO (66.67 $\pm 62.25 \mathrm{~mm} 3$ for Navitoclax group, $n=8$, vs. $152.9 \pm 91.01 \mathrm{~mm} 3$ for PBS group, $\mathrm{n}=8$, $\mathrm{P}=0.040$ ). The Navitoclax treated group showed better functional performance using mNSS after 8 days compared with the PBS group and these effects persisted for 15 days $(P=0.028)$. In addition, Navitoclax reduced the expression of inflammatory end-products: cyclooxygenase-2 (COX-2) and interleukin-1 (IL1b) following MCAO.

\section{Conclusions}

We have demonstrated that intravenous Navitoclax treatment improved outcomes after focal ischemia in the brain via a reduction of inflammation. The Navitoclax, senolytic drug, could be a candidate as the novel therapeutic strategies for cerebral ischemia. 


\title{
Poster 206
}

\section{Evaluating the Association of Stroke Penumbra and the Development of Postischemic Stroke Epilepsy Using Computed Tomography (CT) and Magnetic Resonance (MR) Perfusion}

\author{
TD Sudhakar, AL Soto, AS Zubair, HY Chen, L. Kuohn, AL Herman, NH Peterson, EG Gilmore, LJ Hirsch, A. \\ Sivaraju, C. Matouk, KN Sheth, JA Kim \\ Yale Neurology, New Haven, CT, United States
}

\section{Background \& Purpose}

Perfusion imaging quantifies salvageable (penumbral) from unsalvageable (core) tissue. This penumbral tissue has increased hyperexcitable potential and may increase PISE risk. We hypothesize that size and location of core and penumbra modify epileptogenic risk and are valuable for assessing post-ischemic stroke epilepsy (PISE).

\section{Methods}

From the Yale Acute Brain Injury Biorepository, we identified PISE patients ( $\geq 1$ seizure 2-12 months poststroke) and non-PISE controls who had day of admission computed tomography (CT) or magnetic resonance (MR) perfusion images. We analyzed Rapid Processing of Perfusion and Diffusion (RAPID) software estimates of cerebral blood flow ( $C B F \leq 30,34 \mathrm{~mL}$ ), time to maximum (tmax $\geq 4,6$, 8- seconds) and mismatch volumes. We assessed core and penumbra involvement within the frontal, parietal, temporal, and occipital lobes. We performed univariable logistic regressions.

\section{Results}

We identified 59 patients (age 59, [SD 15], 52\% F), of which 19 (32\%) developed PISE [median NIHSS = 16 (IQR 11-20)]. We observed larger penumbra (tmax $\geq 4$ seconds) and mismatch volumes(CBF $\leq 34 \mathrm{~mL}$, tmax $\geq 4$ seconds) in PISE patients, but the differences were not statistically significant ( $p=0.08$ for both measures). Univariable logistic regression of core infarct within the parietal lobe significantly increased PISE risk (CBF $\leq 30 \mathrm{~mL}, \mathrm{OR}=3.00,95 \% \mathrm{Cl}=1.00-9.48, \mathrm{p}=0.05)$.

\section{Conclusions}

In this exploratory study, we did not find an association between core or penumbra volumes for PISE development. However, parietal lobe involvement increased PISE risk. Future studies from larger patient cohorts may help to better define the relationship between ischemic penumbra and PISE. 
Poster 207

\section{High Blood Pressure Variability during Interfacility Air Transport is Associated with Worse Outcomes in Large-vessel Occlusion Stroke}

Jessica Kobsa, Ayush Prasad, Alexandria L. Soto, Ilayda Top, Smeer Salam, Gary Valeriano, David Kugler, Jonathan Berkowitz, Darko Quispe-Orozco, Mudassir Farooqui, Cynthia Zevallos, Ka-Ho Wong, Lauren Sansing, Joseph Schindler, Charles Matouk, Santiago Ortega-Gutiérrez, Adam de Havenon, Nils H. Petersen

Yale School of Medicine, Division of Neurocritical Care and Emergency Neurology, New Haven, CT, United States

\section{Background \& Purpose}

High systolic blood pressure variability (BPV) after endovascular thrombectomy has been associated with an increased risk of hemorrhagic transformation and unfavorable functional outcomes. However, little is known about the clinical significance of BPV during the hyperacute period after large-vessel occlusion stroke. This study investigated the association between BPV during interfacility air transport and 90-day functional outcome.

\section{Methods}

From prospectively maintained stroke registries, we identified patients who were transferred via helicopter from an outside hospital to one of three comprehensive stroke centers (CSC) for possible endovascular thrombectomy between 2016 and 2021. We collected inflight BP and hemodynamic data for each patient and calculated BPV as the standard deviation of all systolic blood pressure (SBP) values recorded during the air transport. Functional outcome was measured using the modified Rankin Scale (mRS) at 90 days. The association of systolic BPV with functional outcome was quantified using ordinal logistic regression.

\section{Results}

The final analysis included 113 patients with anterior or basilar LVO stroke (mean age $70 \pm 13$ years, $50 \%$ female, mean CSC admission NIHSS 15). The median number of BP measurements during air transport was $6($ IQR 4, 8) with an average SBP of $144.9 \mathrm{mmHg}$ (SD $23.0 \mathrm{mmHg}$ ). A good outcome (mRS 0-2) was achieved by 42 patients (37\%). Systolic BPV was higher among patients with poor vs. good outcome (12.9 vs. $10.0 \mathrm{mmHg}, \mathrm{p}=0.14$ ). Higher BPV during interfacility air transport was independently associated with a worse $\mathrm{mRS}$ score at 90-days after adjusting for age, number of BP values, and reperfusion status (aOR per $3 \mathrm{mmHg}$ increase in BPV 1.16, 95\% Cl 1.0, 1.3, p=0.03).

\section{Conclusions}

Higher BPV during interfacility air transport was associated with an increased risk of worse 90-day functional outcome. These results suggest that BP management strategies targeting high BPV during interfacility transport warrant further prospective investigation. 
Poster 208

Impact of Early COVID-19 Pandemic Upon Telestroke Volume and Characteristics of Ischemic Stroke Presentation

Chad M. Miller, Asma Moheet, Shelly Clark, Karen Deli

Teladoc Health Physician Services, Santa Barbara, CA, United States

\section{Background \& Purpose}

The COVID-19 pandemic has had significant impact on healthcare delivery. Case series have suggested the number of patients emergently presenting with ischemic stroke (IS) early in the pandemic was reduced. Description of the subsequent impact on timing of presentation, eligibility for revascularization therapy, and disease severity are lacking. This study seeks to evaluate the impact of the early pandemic patient concerns upon IS presentation among a large national acute teleneurology cohort.

\section{Methods}

Patients presenting with TIA or IS were identified from hospitals served by a large national acute teleneurology practice for two distinct time periods, March 1 - May 15, 2020 (COVID SURGE), and the corresponding period in 2019 (CONT 2019). Patient demographics and data related to presentation and treatment were extracted from a singular electronic medical record used for all patient documentation. Means and proportions of identified end points were compared between the two groups using Twotailed 2 sample T Tests and 2 Sample Proportion Tests.

\section{Results}

A total of 4,448 patients met study criteria (1896 for COVID SURGE, 2552 for CONT 2019). A $25.7 \%$ reduction in total volume was noted during COVID SURGE and a significant decreased in daily IS volume [24.9 (95\% Cl 23.2-26.7) vs. $33.4(95 \% \mathrm{Cl} 31.9-34.9)]$. Patients presenting during the COVID SURGE presented later (15 hr. 10 min. vs 10 hr. 48 min., p=0.03) and had higher NIHSS [5.4 (95\% Cl 5.1-5.8) vs. $4.6(95 \% \mathrm{Cl} 4.3-4.9) \mathrm{p}<0.001)$. Intravenous thrombolysis rates were similar between both groups $(8.6 \%$ vs. $8.7 \%, p=0.91)$. Disparity in IS volume differential was geographically heterogenous.

\section{Conclusions}

The volume of IS patients presenting during the early COVID-19 surge was reduced with delayed presentation and greater severity of disease. These findings have significant impact on treatment options and need for public education during this and future healthcare crises. 
Poster 209

\section{Ischemia Induces Cathepsin L Secretion in Cerebrovascular Endothelial Cells}

Xuefang Ren, Heng Hu, Huimahn A. Choi, Aaron M. Gusdon, Hua Chen, Atzhiry S. Paz, Glenda L. Torres, Jude P J Savarraj, Jaroslaw Aronowski, Ryan S. Kitagawa

Neurosurgery, Houston, TX, United States

\section{Background \& Purpose}

Ischemic stroke is caused by occlusion of a cerebral artery, which leads to insufficient energy supply to the brain region affected causing neuronal death and neurological damage. Stroke initiates microvascular injury and induces blood-brain barrier (BBB) dysfunction. Cathepsin L, a potent papain-like protease, is known to degrade major structural components of the BBB including perlecan, collagen IV, laminin, and fibronectin. The role of Cathepsin- $L$ in stroke has not been explored.

\section{Methods}

Using a transient middle cerebral artery occlusion (tMCAO, 60 min occlusion) model, we measured Cathepsin-L in plasma of mice at 6-hours post-stroke and compared levels to sham controls. In order to further identify the cellular source of Cathepsin L, we measured Cathepsin-L in cultured cerebrovascular endothelial cells (CECS) and performed an oxygen-and-glucose-deprivation (OGD) assay, which is an invitro ischemia model. We further compared Cathepsin-L levels in plasma from 30 stroke patients to 5 matched controls.

\section{Results}

In our experimental in vivo model, we found that plasma Cathepsin-L levels were elevated at 24 hours post-tMCAO ( $14225 \pm 3297.17$ vs. $22516 \pm 7469.16 ; n=8$ /group; $p=0.0123$ ). In three independent OGD experiments, we found that Cathepsin-L activity was increased in supernatant at 3-hours ( $1462.67 \pm 898.25$ vs. $10873 \pm 352.46 ; p=0.0239)$ and 6 -hours ( $1434.33 \pm 799$ vs. $11400.67 \pm 343.05$; $\mathrm{p}=0.0062)$ but decreased in cell lysates of cultured CECs at 3 -hours $(17289.33 \pm 1178.04$ vs. $14188.33 \pm 952.8 ; p<0.0001)$ and 6 -hours (18288.33 \pm 1096.58 vs. $12311.67 \pm 1136.26 ; p<0.0001)$. In clinical samples from stroke patients, Cathepsin $L$ showed a trend toward higher levels in plasma from stroke patients $(24.13 \pm 41.76)$ compared with controls $(11.40 \pm 8.71)$.

\section{Conclusions}

We have shown Cathepsin- $\mathrm{L}$ is elevated in both stroke models and trended to be higher in stroke patients. Further we have identified that CECs produce excess Cathepsin-L in hypoxic conditions. The known biologic role of Cathepsin- $L$ as a protease degrading structures important in maintaining BBB integrity makes it an interesting marker warranting further investigation. 


\section{Poster 210}

\section{Midline shift greater than $3 \mathrm{~mm}$ independently predicts outcome after acute ischemic stroke}

Morgan E. McKeown, Ayush Prasad, Jessica Kobsa, Ilayda Top, Kevin N. Sheth, Nils Petersen, W. Taylor Kimberly, Matthew B. Bevers

Division of Neurocritical Care, Brigham and Women's Hospital, Boston, MA, United States

\section{Background \& Purpose}

Cerebral edema is associated with worse outcome after acute stroke. In clinical settings, mass effect due to edema is most commonly measured as midline shift (MLS). However, the minimum clinically meaningful MLS is not known. The aim of this study was to identify a threshold level of shift that impacts clinical outcome after acute stroke, with and without endovascular thrombectomy.

\section{Methods}

Patient-level data from six acute stroke clinical trials were pooled to create a cohort of 1,524 patients (Cohort A). A second cohort of patients who underwent endovascular thrombectomy was derived from comprehensive stroke center databases at two academic referral centers (730 patients, Cohort B). MLS was extracted from the original trial data or was measured on computed tomography or magnetic resonance imaging a median of 33.8 hours (IQR 17.3, 73.0) after stroke. Logistic regression was performed to determine significant predictors of poor outcome and the minimum MLS that remained independent.

\section{Results}

The presence of MLS was a predictor of poor outcome in Cohort A, independent of baseline clinical and demographic factors (adjusted OR $2.5[1.7,3.7] ; p<0.001)$. The effect size of MLS in patients who were treated with thrombectomy was similar (aOR $2.2[1.2,4.2] ; p=0.013)$. Examining the full range of MLS values identified $>3 \mathrm{~mm}$ as the key threshold that predicted poor outcome, adjusting for other clinical variables.

\section{Conclusions}

These results show that an MLS value $>3 \mathrm{~mm}$ is clinically significant, both with and without thrombectomy. These findings may have relevance for the design and interpretation of future trials of anti-edema therapies. 


\section{Poster 211}

\section{PECAM Blockade Modulates Neutrophil Infiltration Pattern in Ischemic Stroke}

Neil Nadkarni, Erika Arias, Raymond Fang, Ayush Batra, William Muller, David Sullivan

McGaw Northwestern Memorial Hospital, Department of Neurology, Chicago, IL, United States

\section{Background \& Purpose}

Current therapies for ischemic stroke focus on reperfusion but do not address the acute inflammatory response. To advance future therapies, a thorough understanding of the precise spatiotemporal underpinnings of leukocyte extravasation and infiltration is necessary. We describe the evolution of the inflammatory response in a mouse transient middle cerebral artery occlusion (tMCAO) stroke model at several time points after reperfusion and the modulation of this response with PECAM blockade.

\section{Methods}

The tMCAO model was employed with 90 minutes of ischemia followed by reperfusion to simulate large vessel occlusion stroke and recanalization. We used wide field and confocal immunofluorescence microscopy to examine the exact distribution of neutrophils with close examination of the leukocyte position with regard to the brain vasculature and the perivascular space.

\section{Results}

The recruitment of neutrophils varied dramatically throughout infarcted tissue, especially at early timepoints. At 12 and 24 hours, neutrophil recruitment and extravasation is predominated observed at the cortical surface. Over 48 to 72 hours, neutrophils are increasingly found deeper into the subcortex. Throughout the infarct (determined with triphenyl tetrazolium chloride staining), neutrophil aggregation was not uniform but rather organized in clusters. At $72 \mathrm{~h}$ post-reperfusion, disrupting leukocyte diapedesis with PECAM function-blocking antibodies restricted leukocytes to within 500 microns of the surface when compared to control ( $n=6$ mice per group, $p<0.01$, Control $46 \% \pm 4.0 \%$; PECAM-1 Ab $62 \%$ $\pm 5.0 \%$ ).

\section{Conclusions}

Our findings demonstrate that neutrophil infiltration evolves over several days following reperfusion. The use of PECAM blockade modulates the natural progression of neutrophils into the infarcted stroke bed. A better understanding of neutrophil spatiotemporal infiltration and its regulators could help inform the next generation of therapeutic interventions. 


\title{
Poster 212
}

\section{Potentially Inappropriate Medication Use in Older Adults is Associated with Poor Health Outcomes after Ischemic Stroke}

\author{
Jennifer M. Giulietti, David J. Lin, Julie A. Dicarlo, Kimberly Erler, Megan E. Barra \\ Massachusetts General Hospital, Department of Pharmacy, Boston, MA, United States
}

\section{Background \& Purpose}

Approximately $88 \%$ of strokes occur in patients $\geq 65$ years old. The American Geriatric Society (AGS) Beers Criteria is a tool developed to help identify potentially inappropriate medication (PIM) use in older adults when equally or more effective, but lower risk alternative therapy, is available. This study seeks to evaluate the prevalence of PIMs and association of PIM use on health outcomes in older adults following acute ischemic stroke (AIS).

\section{Methods}

This was a retrospective analysis of patients $\geq 65$ years old with AIS cared for in a post-stroke recovery clinic from 07/2017 to 08/2019. Medications prior to admission (PTA), discharge, and through 6-month follow up were evaluated for PIM use per the 2019 AGS Beers Criteria. Modified Rankin Score (mRS) at 6 months was utilized to compare potential impact of PIM use on favorable (mRS 0-2) versus unfavorable outcomes (mRS 3-6). Mann-Whitney U Test was used for between group comparison and McNemar Test for paired outcomes.

\section{Results}

Forty-one patients were included in the analysis. Nineteen(46\%) of patients were prescribed a PIM PTA, which increased to $24(59 \%)$ by the time of acute hospital discharge $(p=0.88)$. Of 31 patients who completed 6-month follow up, 17(55\%) reported taking at least one PIM. At 6 months, when compared to patients with good outcomes (mRS 0-2; 17/31), patients with poor outcomes (14/31) were more likely to take scheduled PIMs ( $p=0.048)$. There was no significant difference between PRN PIM use $(p=0.43)$. At 6 months, the most commonly prescribed PIMs were PPIs (9/31), NSAIDs (8/31), and sliding scale insulin monotherapy (3/31).

\section{Conclusions}

Utilization of medications associated with increased risk in older adults is common and associated with poor health outcomes in post-AIS care. Future validation in larger cohorts, investigation on cognitive outcomes, as well as assessment of medication profiles to determine when PIM use could be appropriate is needed. 


\section{Poster 213}

\section{Predicting Length of Stay For Acute Stroke Patients Using 24 Hours Of Hemodynamic Features}

Zachary Murphy, Michael Ainsworth, Alex Hepp, Varun Naga, Athena Olszewski, Kirby Gong, Elizabeth Zink, Joseph Greenstein, Raimond Winslow, Mona Bahouth

Department of Biomedical Engineering, Johns Hopkins University, Baltimore, MD, United States

\section{Background \& Purpose}

Stroke is a leading cause of death and disability worldwide. During the acute stroke period, there is a disruption of the blood-brain barrier and cerebral blood flow autoregulation, which results in a direct relationship between hemodynamics and brain perfusion. Improved understanding of the relationship between hemodynamic trends and clinical outcomes could provide predictive insight. Hospital length of stay can serve as a surrogate clinical outcome and the ability to predict length of stay would assist in the coordination of care. The goal of this work was to predict hospital length of stay using the patient's demographic information and hemodynamic profile.

\section{Methods}

We conducted a retrospective cohort study for stroke patients admitted to an urban comprehensive stroke center between 2016-2019. Data included hemodynamic parameters such as pulse, temperature, blood pressure, respirations, and oxygen saturation as well as demographics and comorbidities. Raw time series data were transformed into features for modeling using the Python package tsfresh. Our primary outcome was length of stay greater than or equal to 7 days. Logistic regression, random forest, and XGBoost models were built using data collected during the first 24 hours after hospital admission. Models were improved through the use of randomized search hyperparameter tuning.

\section{Results}

Of the data collected, 2,025 stroke patients met the inclusion criteria. Using an XGBoost classifier we obtained an AUC of 0.839 , with the optimal operating point achieving an accuracy of 0.79 , sensitivity of 0.77 , and specificity of 0.80 . The precision-recall curve for this model had an AUC of 0.774 .

\section{Conclusions}

The model suggests that hospital length of stay can be predicted with reasonable accuracy using clinical data from the first 24 hours of hospitalization. The application of this model could augment the care coordination process and provide a categorization of patient recovery trajectory. 


\section{Poster 214}

\section{Pre-stroke Blood Pressure Variability Affects Blood Pressure Variability in the Acute Phase after Mechanical Thrombectomy}

Alexandria L. Soto, Ayush Prasad, Jessica Kobsa, Sreeja Kodali, Darko Quispe-Orozco, Mudassir Farooqui, Cynthia Zevallos, Lauren Sansing, Joseph Schindler, Charles Matouk, Kevin N. Sheth, Adam DeHavenon, Santiago Ortega-Gutiérrez, Nils H. Petersen

Yale School of Medicine, Department of Neurology, New Haven, CT, United States

\section{Background \& Purpose}

High blood pressure variability (BPV) after endovascular thrombectomy has been associated with hemorrhagic transformation and worse neurological outcomes. However, whether BPV is an epiphenomenon of the stroke itself or causally related to the outcome remains unresolved. This study aimed to evaluate if a relationship exists between pre-and post-stroke BPV in patients with LVO stroke.

\section{Methods}

From our prospective stroke registry, we identified patients who had an anterior circulation LVO, underwent EVT, and had at least three blood pressure measurements recorded in the electronic medical record in the six months prior to their stroke admission. All patients had repeated time-stamped blood pressure data recorded for the first 72 hours after thrombectomy. Using the standard deviation of systolic BP, we calculated BPV for each patient and separated patients into tertiles based on their poststroke BPV. The relationship between pre-stroke BPV and post-EVT BPV was analyzed using an ordinal logistic regression and Spearman's rank correlation analysis.

\section{Results}

One hundred and seven patients were included in our analysis (mean age $71 \pm 16.9$ years, mean admission NIHSS 16 \pm 6 , median pre-stroke BP measurements 22 (IQR 7.0-56.6)). Pre-stroke BPV gradually increased for patients with higher post-EVT BPV tertiles (tertile $1=12.23( \pm 4.96) \mathrm{mmHg}$, tertile $2=$ $14.93( \pm 5.03) \mathrm{mmHg}$, tertile $3=16.78( \pm 6.28) \mathrm{mmHg}, \mathrm{p}=0.003)$. A positive correlation was observed between pre-stroke BPV and post-EVT BPV $(p=0.004, R=0.28)$. After adjusting for age and admission NIHSS, pre-stroke BPV was significantly associated with post-EVT BPV tertile membership (OR 1.1, 95\% Cl 1.05-1.21, $p<0.001)$.

\section{Conclusions}

High pre-stroke BPV is correlated with high post-EVT BPV. Although larger, prospective studies are needed to provide definitive evidence of this relationship, our work suggests that high post-EVT BPV may be related to an underlying biological phenomenon and not merely a consequence of the stroke itself. Individuals with high BPV may benefit from more intensive blood pressure management after EVT. 


\section{Poster 215}

\section{Proxy Measures to Approximate Post-Stroke Modified Rankin Scale Score: A Systematic Literature Review}

Olivia S. Costa, Mark J. Alberts, Mary J Christoph, Belinda Lovelace, Craig I. Coleman

Department of Pharmacy Practice, University of Connecticut School of Pharmacy, Storrs, CT, United

States

\section{Background \& Purpose}

Modified Rankin Scale (mRS) scores are used to assess post-stroke disability in studies but are often irregularly measured or unavailable in routine clinical practice. We performed a systematic literature review to identify proxy measures that could be used to approximate post-stroke mRS scores in retrospective studies.

\section{Methods}

Bibliographic searches were performed of Medline and Embase from Jan 2000-Dec 2020. Eligible studies evaluated the association between $\geq 1$ proxy measure and $\mathrm{mRS}$ score 30 or more days post-stroke.

Associations between proxies and ordinal mRS score were assessed based upon correlation coefficients ( $r< \pm 0.50$ weak/none, \pm 0.50 to \pm 0.70 moderate, $> \pm 0.70$ strong), using area under-the-curve (AUC) $(<0.70$ poor, 0.7 to 0.80 acceptable, $>0.80$ as good/excellent) and/or positive and negative predictive values (PPV and NPV) for unfavorable mRS score (defined as scores $\geq 2$ ).

\section{Results}

We included 9 studies (median sample size=1699; range 530-6809 patients). Studies evaluated hometime ( $n=7$, number of follow-up days spent at home starting at diagnosis), discharge destination ( $n=2$, home vs. rehabilitation or skilled nursing facility) and/or index hospital length-of-stay $(n=1)$ as potential proxies for $\mathrm{mRS}$ score post-ischemic $(n=8)$ and/or hemorrhagic $(n=2)$ stroke. Moderate-to-strong inverse correlations were observed between home-time and ordinal mRS score $(r=-0.69$ to -0.86 , $\mathrm{p}<0.001$ for all) at 90-days, although home-time was less able to differentiate between increasing and neighboring $\mathrm{mRS}$ scores starting at 3 . Shorter home-time was a strong predictor of unfavorable $\mathrm{mRS}$ at 90-days ( $A \cup C=0.84)$. Discharge to a location other than home was strongly correlated $(r=0.71 ; p<0.001)$ with, and accurately predicted ( $\mathrm{PPV}=95 \%, \mathrm{NPV}=4.5 \%$ ) unfavorable $\mathrm{mRS}$ score $>3$ at 90 -days (prevalence $=42$ to $50 \%$ ). These findings remained consistent when assessed through 365 -days poststroke and when alternative definitions of unfavorable $\mathrm{mRS}$ score ( $\geq 3$ or $\geq 4$ ) were utilized. Length-ofstay was weakly correlated with $\mathrm{mRS}$ score $(r=0.29, p<0.0001)$.

\section{Conclusions}

Home-time and discharge destination may be reasonable proxies for post-stroke mRS score. 
Poster 216

\title{
Rules-Based Natural Language Processing to Extract Features of Large Vessel Occlusion and Cerebral Edema from Radiology Reports in Stroke Patients
}

\author{
Rajat Dhar, Zohair Siddiqui, Aaron Corbin, Kunal Bhatia
}

Neurology; Washington University in St. Louis School of Medicine, Saint Louis, MO, United States

\section{Background \& Purpose}

A growing proportion of stroke patients undergo multimodal CT to evaluate for large vessel occlusion (LVO) and suitability for acute interventions. Accurately identifying and phenotyping large cohorts of LVO patients will empower big data research. We present a natural language processing (NLP) approach to automatically extract meaningful LVO phenotypes from the radiology reports of stroke patients.

\section{Methods}

Rules-based NLP software was developed in Python to extract presence and location of LVO from fulltext CTA reports and RAPID core/penumbra volumes from CTP reports. A single-institution cohort from 2018-2019 was used for algorithm development and the following year for validation. Performance was compared with manual annotations using recall (sensitivity), precision (predictive value) and F1 score (balanced accuracy). All follow-up CT reports in those with LVO were evaluated for documentation of cerebral edema and midline shift.

\section{Results}

The NLP algorithm had $94 \%$ recall and $88 \%$ precision (F1 0.91) for identifying occlusions, seen in 153/577 reports in the development cohort. It could accurately label occlusion side ( $96 \%$ recall, F1 0.95) and differentiate $M 1$ from $M 2$ segment occlusions (95\% recall, F1 0.93). In the validation cohort, it retained $88 \%$ recall for occlusions (F1 0.89), seen in 78/400 reports. Core/penumbra volumes were extracted correctly in 150/153 (98\%) of CTP reports. NLP identified 41 of 125 LVO patients in the initial cohort with radiographic edema on follow-up CT: these cases had larger core (32 vs. 11-ml) and penumbra volumes (107 vs. 74-ml) and were more likely to have NLP-identified proximal occlusion (OR 2.04, p=0.11); they also had a higher risk of developing clinically-adjudicated malignant edema (OR 26, 95\% CI 7.2-96.2).

\section{Conclusions}

We present an accurate NLP-based method of extracting several features relevant to LVO and cerebral edema from radiographic reports. After external validation, it could be used to mine large databases and extract targeted patient cohorts with LVO, identifying those with complications. 


\title{
Poster 217
}

\section{Serial MRI Brain Findings in COVID-19 Patients with Neurologic Manifestations}

\author{
Ayush Batra, Sareen Ali, Steven C. Hoffman, Jeffery R. Clark, Nathan A. Shlobin, Tulsi R. Patel, Anthony \\ Kang, Patrick H. Lim, Zachary S. Orban, Igor J. Koralnik, Eric M. Liotta
}

Northwestern University Department of Neurology, Chicago, IL, United States

\section{Background \& Purpose}

A growing body of literature has identified acute neuroimaging findings associated with neurological manifestations in hospitalized COVID-19 patients. Limited studies have examined neuroimaging in a serial fashion in these patients. We sought to characterize the temporal evolution of neuroimaging findings in hospitalized COVID-19 with neurologic manifestations who underwent sequential brain magnetic resonance imaging (MRI).

\section{Methods}

We identified patients requiring hospitalization for COVID-19 at a larger tertiary healthcare system for whom any MRI brain was performed between March 5th and November 9th 2020. Patients were included if an initial MRI was performed for neurologic symptoms within 14 days prior to or any time after confirmed COVID-19, with follow-up brain MRI performed at least 14 days apart. Patients were excluded if neuroimaging indication was attributable to a preexisting neurologic condition or if sequential MRI was performed for follow-up of a primary/metastatic brain tumor.

\section{Results}

207 patients underwent initial brain MRI, of whom 10 were identified with serial brain MRIs meeting study inclusion criteria. On initial brain MRI, 5/10 patients had evidence of acute or subacute infarcts, 9/10 patients had non-specific fluid-attenuated inversion recovery (FLAIR) signal abnormalities, and 7/10 had evidence of microhemorrhages on susceptibility weighted imaging (SWI)/gradient echo sequences (GRE). No abnormal meningeal enhancement was identified. Follow-up brain MRI demonstrated new findings, including: $2 / 10$ patients with acute or subacute infarcts, $7 / 10$ patients with non-specific FLAIR signal abnormalities, and 6/10 patients with microhemorrhages on SWI/GRE sequences. No meningeal enhancement was identified on follow-up MRIs.

\section{Conclusions}

Sequential brain MRIs of hospitalized COVID-19 patients with neurologic symptoms demonstrated findings consistent with acute to subacute cerebrovascular disease including subacute infarcts and microhemorrhages. These findings suggest that clinically silent cerebrovascular events may be more prevalent than currently recognized in hospitalized COVID-19 patients and those with neurologic manifestations may warrant closer follow-up with serial imaging. 


\section{Poster 218}

\section{Virtual High Energy Monochromatic (190keV) CT ASPECTS Predicts Malignant Cerebral Edema after Large Hemispheric Infarction}

William Zimmerman, Melissa Pergakis, Nicholas Morris, Jamie Podell, Wan-Tsu Chang, Melissa Motta, Hegang Chen, Uttam Bodanapally, Neeraj Badjatia, Gunjan Parikh

University of Maryland Medical Center, Department of Neurology, Neurocritical Care, Baltimore, MD, United States

\section{Background \& Purpose}

Imaging biomarkers of malignant cerebral edema (MCE) after large hemispheric infarct (LHI) are understudied. We hypothesize that acute virtual high-energy monochromatic (190keV) CT imaging (VMI) enables earlier detection of secondary injury from MCE.

\section{Methods}

This is a study of consecutive LHI patients who had VMI within 10 hours of reperfusion from May 2020 to March 2021. We excluded patients with parenchymal hematoma-type 2 transformation.

Retrospective analysis of novel VMI variables included Alberta Stroke Program Early CT Score (ASPECTS), iodine density (ID) of coinciding ASPECTS locations, and average maximum ID. Primary outcome was $\mathrm{MCE}$, defined as clinical worsening (increase in NIHSS $\geq 4$ ) or radiographic herniation (midline shift > $5 \mathrm{~mm}$ ). Secondary outcomes included petechial hemorrhagic transformation, need for decompressive craniectomy (DC), and increased intracranial pressure (ICP) treatment. Fisher's exact test and Wilcoxon Rank Sum test were used for univariate analysis. The outcomes were analyzed by logistic regression model.

\section{Results}

Thirty-eight LHI patients with mean age of $66.8 \pm 15.3$ years and median initial NIHSS of 21.5 [IQR 16,25] met criteria. Twelve patients (31.6\%) developed MCE on mean post stroke day $2.1 \pm 1.6$. VMI ASPECTS, difference between initial standard energy CT and VMI ASPECTS, ASPECTS insular, M1, M5 and M6 ID were associated with MCE. Max ID was not associated with MCE. VMI ASPECTS correlated with hemorrhagic transformation but not with ICP treatment or need for DC. In a logistic regression analysis, VMI ASPECTS was a predictor of MCE (aOR, 0.45;95\% Cl: $[0.6,0.76] ; p=0.0033)$ and petechial hemorrhage (aOR, 0.6; 95\% $\mathrm{Cl}$ : [0.40, 0.91]; $p=0.0155$ ) after adjusting for age, sex, initial NIHSS, admission glucose, and tPA administration, with a mean AUROC of $0.888 ; 95 \% \mathrm{Cl}[0.7635,1.0000]$ and $0.833 ; 95 \% \mathrm{Cl}[0.7042,0.9624]$ respectively.

\section{Conclusions}

Acute VMI ASPECTS predicts MCE after LHI and may improve models predicting secondary brain injury after stroke. 


\section{Poster 219}

\section{Whole-Blood Replacement Therapy Rescues the Brain from Acute Stroke Damage}

Xuefang Ren, Heng Hu, Huimahn A. Choi, Aaron M. Gusdon, Ryan S. Kitagawa, James W. Simpkins

University of Texas Health Science Center at Houston, Houston, TX, United States

\section{Background \& Purpose}

Background: Stroke triggers an intense peripheral/systemic response. This maladaptive peripheral response is characterized by changes in the blood which contribute to leukocyte infiltration across a compromised blood-brain-barrier (BBB) causing neuroinflammation, further brain injury, and worse outcomes. We have recently describing the beneficial effects of blood replacement (BR) therapy in a mouse stroke model. By removing whole-blood from stroke mice and subsequently replacing blood from non-injured mice, we demonstrated preservation of the BBB, decreased stroke volumes, and improved outcomes (Nature Communications 11(1):4078, 2020). To further flow this line of work and develop a more translatable model, we performed BR-therapy through simultaneous blood-removal and bloodtransfusion strategy to evaluate the effect of BR-therapy on outcomes in stroke mice. Simultaneous removal-transfusion can allow for safer BR-therapy for larger volumes of blood.

\section{Methods}

Methods: A murine transient middle cerebral artery occlusion (tMCAO, 90 min occlusion) stroke model was utilized in the study. We developed a micro-sized-3-way-stopcock for BR-therapy and applied a removal-transfusion approach with $500 \mu \mathrm{l}$ of blood for stroke mice. We measured outcomes including neurological deficits and infarct volume by TTC-staining at 23-hours post-stroke. Student's t-test for infarct volumes and Mann-Whitney test for neurological deficits were used for statistical significance.

\section{Results}

Results: Our results show that this novel approach profoundly improves outcomes after stroke. BRtherapy significantly reduces infarct volumes including cortex (control $32.15 \pm 11.14, n=5$ vs. BR-therapy 11.17 $\pm 5.15, n=5 ; P=0.0051$ ), striatum (control 151.28 \pm 17.65 , vs. BR-therapy $91.98 \pm 36.91 ; P=0.012$ ), hemisphere (control $48.19 \pm 12.46, n=5$ vs. BR-therapy $22.29 \pm 8.96, n=5 ; P=0.0054$ ). We also demonstrated a profound reduction in neurological deficits (control $3 \pm 1.53, n=7$, vs BR-therapy $1 \pm 0.71$, $\mathrm{n}=5 ; \mathrm{P}=0.0278$ ) at 23-hours post stroke.

\section{Conclusions}

Conclusion: We have developed a new method of BR-therapy and confirmed robust improvement in stroke outcomes with BR-therapy. Simultaneous blood removal-transfusion will allow for higher volumes of blood to be exchanged and is a more translatable treatment strategy. 


\title{
Poster 220
}

\section{Cefepime Serum Trough Concentration Varies Significantly in ICU Patients Despite Appropriate Dose Adjustment for Renal Function and Indication}

\author{
Alice Wang, Minkey Wungwattana, David J. Gagnon, Richard R. Riker, Kristina Connolly \\ Maine Medical Center, Pharmacy Department, Portland, ME, United States
}

\section{Background \& Purpose}

Elevated cefepime serum trough concentration has been associated with neurotoxicity; few data describe this relationship in critically ill patients. The objectives of this study were to compare administered cefepime dose to that recommended in the prescribing information and to assess trough concentration and its association with neurotoxicity.

\section{Methods}

This single-center, retrospective cohort study included consecutive critically ill adults who received cefepime with a measured serum trough concentration. Dose appropriateness included both renal function and indication per the prescribing information. Neurotoxicity was determined with blinded chart review by two investigators. Data are reported as median (IQR).

\section{Results}

Twelve patients were included, with an age of 60 (57-73) years, estimated $\mathrm{CrCl}$ of 146 (51-165) mL/min, and cefepime dose of 5 (3-6) g/day. Cefepime trough concentration was 57 (23-85) mg/L, drawn 2.6 (1.5-3) days after cefepime initiation. Eight troughs (67\%) were above $30 \mathrm{mg} / \mathrm{L}$. Cefepime dose was consistent with the prescribing information for renal function and indication in 11/12 (92\%) patients. Three patients (25\%) experienced neurotoxicity, with a median dose of 1 (1-3.5) g/day and trough of 76 (47-108) $\mathrm{mg} / \mathrm{L}$. Neurotoxicity was described as delirium and encephalopathy in all 3 patients. Four (33\%) patients had renal dysfunction and a lower cefepime dose compared to patients without renal dysfunction: 2.5 (1-4.5) versus 6 (4.8-6) g/day, $p=0.028$. Despite appropriate renal dose adjustment, cefepime serum trough concentration trended higher in patients with renal dysfunction (123 [83-199] vs. 44 [23-70] mg/L, $\mathrm{p}=0.15$ ), as did neurotoxicity incidence ( $50 \%$ vs. $12.5 \%, p=0.24$ ).

\section{Conclusions}

Despite high dosing compliance with cefepime's prescribing information for renal function and indication, $25 \%$ of patients developed neurotoxicity. Cefepime doses recommended in its prescribing information may not be appropriate for critically ill patients, especially with kidney injury. Additional studies are needed to investigate cefepime dosing and therapeutic drug monitoring in the ICU. 
Poster 221

\section{Characteristics and Outcome of Patients with Subdural Hematoma on Anticoagulation versus No Anticoagulation}

\section{Lalith Premachandra, Farid Sadaka}

Mercy Hospital St Louis/ Critical Care Medicine Department, St. Louis, MO, United States

\section{Background \& Purpose}

Subdural hematoma (SDH) represents one type of intracranial mass lesion. Because the use of anticoagulants is increasing, this study aims to compare characteristics and outcomes of patients with $\mathrm{SDH}$ receiving anticoagulation (AC) to those not on anticoagulation (NAC).

\section{Methods}

We performed a retrospective chart review of patients admitted with SDH to our hospital between 01/2012 and 03/2021. The following data were collected: Age in years, Glasgow coma score (GCS) on admission, traumatic vs spontaneous, and indication for surgery (a thickness greater than $10 \mathrm{~mm}$ [OR1] or a midline shift (MLS) greater than $5 \mathrm{~mm}$ on CT [OR2], or asymmetric or fixed and dilated pupil [OR3]), and whether they underwent surgical evacuation (S). Outcome was evaluated at hospital discharge on the modified Rankin scale (range, 0 [normal] to 6 [death]) with $0-3$ considered as good functional outcome).

\section{Results}

We identified a total of 322 patients (74 in the AC group and 248 in the NAC group). Compared to the NAC group, the AC group had similar age ( $73+/-13$ vs $71+/-15, p=0.3)$, similar GCS ( $13.2+/-2.8$ vs $13.8+/-3.0, p=0.1)$, higher $\%$ with spontaneous SDH (70\% vs $56 \%, p=0.03)$, similar $\%$ with OR1 (74\% vs $70 \%, p=0.5$ ) , OR2 ( $38 \%$ vs $48 \%, p=0.1$ ) and OR3 indications ( $8.0 \%$ vs $3.2 \%, p=0.07$ ), and similar $\%$ who underwent surgical evacuation ( $46 \%$ vs $57 \%, p=0.1$ ). There was no difference in functional outcome between the AC group and NAC group (mRS 0-3, 74\% vs 77\%, $p=0.6$ ).

\section{Conclusions}

In our single center study, patients with SDH and on AC had more spontaneous bleeds with similar percentages undergoing surgical evacuations and similar outcomes compared to patients not on AC. 


\title{
Poster 222
}

\section{Delirium Screening In The Neuro ICU: A Retrospective Study Evaluating The Confusion Assessment Method For The ICU And Intensive Care Delirium Screening Checklist}

\author{
Nicholas G. Panos, Hannah H. Breit, Kristen Fisher, Amy Blackwood, George Michalopoulos, Ivan Da \\ Silva, Sayona John \\ Rush University Medical Center, Chicago, IL, United States
}

\section{Background \& Purpose}

The Intensive Care Delirium Screening Checklist (ICDSC) and Confusion Assessment Method for the ICU (CAM-ICU) are two evidence based screening tools available to critical care clinicians to detect delirium. However, studies comparing these delirium screening tools in a broad population of neurocritically ill patients have not been performed. The aim of this study is to determine whether the CAM-ICU and ICDSC provide an accurate assessment of delirium when compared to Diagnostic and Statistical Manual of Mental Disorders (DSM-V) criteria used by a neurointensivist.

\section{Methods}

All patients admitted to the neurosciences ICU (NSICU) beginning January 1, 2019 through January 1, 2020 were identified for inclusion into the study. Upon admission to the NSICU, nurses and neurointensivists screened for delirium using the ICDSC, CAM-ICU and DSM-V for the duration of the ICU stay. To be included in the analysis, patients needed at least 1 screening assessment with ICDSC, CAMICU, and DSM-V documented on the same day during the ICU stay. Agreement of the three detection tools was assessed using Fleiss' kappa.

\section{Results}

A total of 206 patients were included in the analysis, with 1442 assessments performed. Prevalence of delirium using DSM-V criteria was $1.6 \%$. When the three tools were compared together there was an agreement of 0.249 . Of the patients with confirmed delirium per DSM-V, 10 were also positive for ICDSC, but not for CAM-ICU. CAM-ICU had a sensitivity of $26 \%$, specificity of $94.5 \%$, and ICDSC had a sensitivity of $69.5 \%$, specificity of $87.6 \%$.

\section{Conclusions}

In a cohort of neurocritically ill patients, the prevalence of delirium was low. In addition, the ICDSC may be more sensitive for detecting delirium in a NSICU. Further prospective studies are needed to determine the utility of delirium screening with the ICDSC and CAM-ICU in the NSICU. 
Poster 223

\section{Evaluation of levetiracetam dosing for seizure prophylaxis in traumatic brain injury}

Alixandra Mann, Kristen Livers, Christine Duff Frick, Emily Sieg, Michelle Bailey, Lindsay Urben

University of Louisville Hospital, Department of Pharmacy, Louisville, KY, United States

\section{Background \& Purpose}

Early onset post-traumatic seizures (PTS) may occur within seven days of traumatic brain injury (TBI). Although levetiracetam is frequently used for this indication, it is not currently recommended in the Brain Trauma Foundation guidelines over phenytoin due to insufficient evidence. There is an existing gap in knowledge regarding the effectiveness of levetiracetam as well as the optimal dosing strategy for early PTS, especially given the high incidence of augmented renal clearance in this population. The objective of this study was to further evaluate levetiracetam dosing strategies for early onset posttraumatic seizure prophylaxis.

\section{Methods}

A single-center retrospective cohort study was conducted utilizing the electronic medical record (EMR) and a trauma database. Our study evaluated the incidence of seizure within seven days of TBI when utilizing low-dose levetiracetam (500 mg twice daily), compared to high-dose levetiracetam (greater than $500 \mathrm{mg}$ twice daily). Only patients who completed a full seven-day course of levetiracetam were included.

\section{Results}

Of the 203 patients included, 149 patients received low-dose levetiracetam and 54 patients received high-dose. The majority of patients had a GCS $<8(53.7 \%)$ and an injury severity score $>15$ on presentation (92.1\%). Twelve of 203 patients (5.9\%) experienced a seizure within seven days of TBI, which is similar to the rate seen in previous studies. Six patients in the low-dose group $(4.0 \%)$ and six patients in the high-dose group (11.1\%) experienced a seizure $(p=0.059)$. There was no statistically significant difference in seizure rate when patients were stratified based on baseline GCS, ISS or mechanism of injury.

\section{Conclusions}

There were no statistically significant differences in seizure rates when comparing low-dose to high-dose levetiracetam. Levetiracetam $500 \mathrm{mg}$ twice daily may be as effective as levetiracetam doses greater than $500 \mathrm{mg}$ twice daily for early onset post-traumatic seizure prophylaxis. 


\section{Poster 224}

\section{Evaluation of the Safety of Rapid Administration of Undiluted High-Dose Intravenous Levetiracetam}

Brittany M. Kasturiarachi, Rashi Krishnan, Diana L. Alsbrook, Brittany Hudson, Hallie Kelley, Caitlin E. Moran, Khalid Alsherbini, Morgan Jones

UTHSC - Neurology, Memphis, TN, United States

\section{Background \& Purpose}

Intravenous (IV) levetiracetam (LEV) is an antiseizure medication (ASM) traditionally given as an intermittent infusion to mitigate any potential adverse effects given its acidic formulation. The process of compounding this may lead to delays in treating status epilepticus cases, which is why administration of undiluted doses is of clinical interest. Prior studies have shown safety of IV doses up to $1000 \mathrm{mg}$; however, assessments of higher doses have not been reported. The goal of this study is to assess the safety of rapid IV administration $(500 \mathrm{mg} / \mathrm{min}$ ) of undiluted LEV $1500 \mathrm{mg}$ across a multi-hospital system.

\section{Methods}

A retrospective analysis was completed with patients who received $1500 \mathrm{mg}$ doses of undiluted IV LEV. We included patients $\geq 18$ years old that received at least one dose of IV LEV $1500 \mathrm{mg}$ from January 2018 to February 2021. Study end points included assessment of hemodynamic disturbance (bradycardia [heart rate less than 50 beats per minute] or hypotension [systolic blood pressure less than $90 \mathrm{mmHg}$ ] within one hour or infusion reaction documented by the nurse within 12 hours of LEV. Data were collected throughout patients' hospitalization. Descriptive statistics were used in the analysis.

\section{Results}

A total 213 doses of $1500 \mathrm{mg}$ of IV LEV were administered to 107 patients. A peripheral line was used for $85.9 \%$ of doses. Approximately half of all doses (57) were administered to patients on the general medicine wards, with the remainder in the intensive care unit or emergency department. Two patients (1.9\%) experienced bradycardia; however, one patient had pre-existing baseline bradycardia. Three patients (3.8\%) experienced hypotension, but in all instances the patients were already receiving vasopressors prior to the dose. There were no cases of infusion reaction.

\section{Conclusions}

Undiluted, rapid administration of IV LEV $1500 \mathrm{mg}$ was well tolerated with rare side effects. 
Poster 225

\title{
External Ventricular Drain Placement Post Andexanet Alfa Bolus Dose
}

\author{
Abdalla A Ammar, Aladine A. Elsamadicy, Mahmoud A. Ammar, Guido J. Falcone, David Y. Hwang, Nils \\ Petersen, Kevin T. Gobeske, Jennifer A. Kim, Rachel Beekman, Julián N. Acosta, Ryan Hebert, Kevin N. \\ Sheth, Charles C. Matouk, Emily J. Gilmore
}

Department of Pharmacy, Yale New Haven Health, New Haven, CT, United States

\section{Background \& Purpose}

Patients with factor-Xa ( $\mathrm{fXa}$ ) inhibitor-associated intracerebral hemorrhage (ICH) may require emergent external ventricular drain (EVD) placement. Andexanet alfa (AA), a fXa reversal agent, is given as a bolus over 15-30 min followed by a 2-hour infusion. This long administration time can delay EVD placement. We sought to evaluate the safety of EVD placement immediately post-AA bolus compared to following 2-hour infusion (post-AAI).

\section{Methods}

This is a retrospective chart review of adult patients ( $\geq 18$ years) admitted to the Yale New Haven Hospital with $\mathrm{fXa}$-associated $\mathrm{ICH}$, who received AA and underwent EVD placement. The primary outcome was the occurrence of a new hemorrhage (tract, extra-axial, or intraventricular hemorrhage) on imaging adjudicated by a neurosurgeon. Secondary outcomes included mortality, ICU and hospital length of stay (LOS) and modified Rankin Score (mRS) on discharge. The primary safety outcome was documented thrombotic event (TE).

\section{Results}

Twelve patients with $\mathrm{fXa-related} \mathrm{ICH}$ were included (post-AA bolus EVD placement, $\mathrm{N}=8$; post-AAI EVD placement, $\mathrm{N}=4$ ). Indication for anticoagulation was mainly atrial fibrillation ( $\mathrm{n}=10,83 \%)$. Median admission Glasgow Coma Score was 6 in both groups. There was no difference between both groups in incidence of new hemorrhages, with one post-AA bolus patient had a small, focal, non-operative extraaxial hemorrhage. Morbidity, measured by mRS, and mortality were higher in the post-AAI group (mRS, post-AA bolus, 4 [4-6] vs. post-AAI 6 [5-6] and post-AA bolus 37.5\% vs. post-AAI 75\%). One patient in the post-AA bolus group had a thrombotic event. There was no difference in hospital LOS (post-AA bolus, 19 days [12-26] vs. post-AAI, 14 days [9-22]) and ICU LOS (post-AA bolus, 10 days [6-13] vs. post-AAI, 11 days [5-21]).

\section{Conclusions}

In our limited sample, we report no differences in incidence of EVD-related tract hemorrhage, extra-axial hemorrhage, or intraventricular hemorrhage between post-AA bolus and post-AAI patients. Larger prospective studies are warranted to validate our findings. 


\section{Poster 226}

\section{Hypoglycemia in the Neuroscience Intensive Care Unit: A Single Unit Quality Improvement Project}

Zehui Wang, Karen Berger, Brooke Broczkowzki, Sabrina Chen, Christian Huang, Chris Koh, Judy Ch'ang, Hooman Kamel

New York Presbyterian Weill Cornell, Department of Neurology, New York, NY, United States

\section{Background \& Purpose}

Hypoglycemia in patients admitted to the neurocritical care unit correlates with higher mortality and worse functional outcomes. We aim to describe the prevalence of risk factors in patients with hypoglycemic events admitted to our neuroscience intensive care unit (NSICU).

\section{Methods}

This was a retrospective review of patients who had at least one documented severe hypoglycemic event defined as blood glucose $<50 \mathrm{mg} / \mathrm{dL}$ from 2012 to 4/20/2020 using the NUCLEUS registry. Four reviewers analyzed patient records to obtain baseline demographics, mortality, length of stay, comorbidities, insulin regimen, diet, and other risk factors such as sepsis and vasopressor use associated with hypoglycemic events in critically-ill patients. Data collection was validated by two independent reviewers.

\section{Results}

A total of 5,125 patients from 7,403 NSICU admissions were evaluated and 50 patients were included in the analysis. Insulin use within 24 hours of the hypoglycemic episode was observed in 36 patients (72\%); 14 (28\%) of patients had a severe hypoglycemia episode despite no insulin exposure. Twenty-five (50\%) patients were switched to nothing-by-mouth (NPO) status or had tube feeds held without concomitant insulin adjustment within 24 hours prior to the severe hypoglycemic event. Also, within 24 hours prior to the hypoglycemic event, 14 (28\%) patients were on a steroid regimen taper, but only 7 of these patients had concomitant insulin adjustment. Eighteen (36\%) patients died during their hospitalization.

\section{Conclusions}

In our study of neurocritical care patients, severe hypoglycemia was observed in the setting of changing a patients' diets to NPO status and being on a steroid regimen taper. This has important implications in preventing iatrogenic hypoglycemic events in patients with acute brain injury. 
Poster 227

Is Andexanet Alfa Compared to 3 Factor- Or 4 Factor- Prothrombin Complex Concentrate a Better Reversal Agent in Patients Presenting with Intracranial Hemorrhage?

Josephine Tenii, Venkatesh Aiyagari, Babu Welch

UT Southwestern Medical Center, Department of Pharmacy, Dallas, TX, United States

\section{Background \& Purpose}

Background: Andexanet Alfa (AA) is FDA approved for reversal of the oral anti-xa inhibitors apixaban and rivaroxaban. Its clinical efficacy in comparison to Prothrombin Complex Concentrate (PCC) is unknown and has significant cost burden.Purpose: Evaluate safety and efficacy or AA compared to 3 Factor or 4 Factor PCC (3F- or 4F-PCC) in patients admitted to an academic hospital with anticoagulant-associated intracranial hemorrhage (AAICH).

\section{Methods}

Methods:Single center, retrospective chart review between January 2018 to February 2021. Inclusion criteria was age $\geq 18$, ICH diagnosis via imaging by neurosurgery, on apixaban and rivaroxaban and treated with AA, 3F- or 4F-PCC 50 units/kg (maximum dose 5000 units). Patients with recent thromboembolism were excluded. Primary outcome: Hematoma stability on 24-hour head CT Scan post reversal Secondary Outcome: Time from order to administration, survival beyond 30 days, hospital and ICU length of stay, inpatient thromboembolism, Modified Rankin Score (MRS) at discharge and discharge destination.

\section{Results}

20 patients met inclusion criteria $(A A=2,3 F-P C C=13,4 F-P C C=3$.) Baseline $G C S$ was similar across groups ( $A A=12,14$ for $3 F-P C C=14$ and 4F-PCC =13). 75\% of patients received anticoagulant within 24 hours of admission. $30 \%$ of patients in PCC groups were on antiplatelets. Stable repeat CT was achieved in $50 \%, 61.5 \%$ and $100 \%$ of AA, 3F-PCC and 4F-PCC groups respectively. Mortality rate for GCS $<7$ or hematoma volume $>60 \mathrm{ml}$ was $50 \%$ in AA versus $23 \%$ in $3 F-P C C$. Discharge MRS (AA = 6, 3F-PCC $=3.5$, $4 \mathrm{~F}-\mathrm{PCC}=4$ ) and destination ( $\mathrm{AA}=50 \%, 3 \mathrm{~F}-\mathrm{PCC}=77 \%, 4 \mathrm{~F}-\mathrm{PCC}=100 \%)$ was favorable with PCC groups. Order time to administration was increased with $A A$, however, no thromboembolism reported between groups.

\section{Conclusions}

In patients with severe ICH, 3F- and 4F- PCC may be preferred with no difference for less severe ICH. 


\title{
Poster 228
}

\section{Monitoring Anti-Xa Levels for Prophylactic Enoxaparin in Neurosurgery Patients}

\author{
Santino Cua, Ciaran Powers Keaton S. Smetana, Casey C. May
}

The Ohio State University Wexner Medical Center, Department of Neurosurgery, Columbus, OH, United States

\section{Background \& Purpose}

Prior studies have demonstrated a reduced risk for venous thromboembolism (VTE) in neurosurgical patients secondary to chemical prophylaxis with heparin and low molecular weight heparin (LMWH). There remains a lack of consensus as to which prophylactic agent to use in the neurosurgical population. Given the ability to monitor LMWH by obtaining anti-Xa serum levels, there is an opportunity to evaluate safety and efficacy. This study seeks to investigate the incidence of VTE and bleeding complications in patients with peak anti-Xa levels within the goal range for VTE prophylaxis (0.2-0.5 $\mathrm{IU} / \mathrm{mL}$ ) compared to those with anti-Xa levels outside of goal range.

\section{Methods}

A single center, retrospective, observational study was conducted on neurosurgical patients receiving enoxaparin for VTE prophylaxis. Significance testing was conducted via Fisher's exact test and independent samples t-test to compare incidence of VTE and bleeding complications between cohorts.

\section{Results}

Eighty-five patients that had a neurosurgical procedure were included in the analysis. Eighteen (21.2\%) had serum anti-Xa levels outside the goal range, with four (4.7\%) supratherapeutic and 14 (16.5\%) subtherapeutic. Six patients (7.1\%) experienced a major bleed post-operatively. Two patients with supratherapeutic anti-Xa levels experienced a major bleed compared to four patients with therapeutic levels ( $p=0.033$ ). Additionally, serum anti-Xa levels were significantly higher in those who experienced major bleeds compared to those who did not $(0.38 \pm 0.13$ vs. $0.28 \pm 0.09, p=0.013)$. Two VTE events occurred; both experienced pulmonary embolisms and had anti-Xa levels in the goal range.

\section{Conclusions}

Monitoring anti-Xa levels for VTE prophylaxis in the neurosurgical population may help prevent major hemorrhages. This data suggests that a higher anti-Xa level may predispose patients to a major hemorrhage post-operatively and that neurosurgical patients may need a lower anti-Xa range than standard hospitalized patients. Further evaluation is needed to identify the goal anti-Xa level for VTE prophylaxis in this population. 
Poster 229

Preliminary Analysis of a Multi-Center Comparison of Anti-Platelet Treatment Strategies for Emergent/Urgent Neuroendovascular Stenting

\author{
Devin Holden, Aaron Cook, Blake T. Robbins, Casey May, the Neurocritical Care Pharmacy Section \\ Research Group.
}

Albany Medical Center Hospital Department of Pharmacy, Albany, NY, United States

\begin{abstract}
Background \& Purpose
Cerebrovascular stent deployment is often used for treatment of patients with acute ischemic stroke, arterial dissection, and cerebral aneurysms. A known risk of cerebrovascular stenting is acute thrombosis and stent stenosis, which is often mitigated by the use of antiplatelet agents. Considerable variability exists in the literature and in practice regarding the appropriate agent, timing of administration in relation to stent deployment, route, and monitoring of antiplatelet therapy in this context. This preliminary analysis of a multicenter retrospective study aimed to evaluate and characterize variation in choice of antiplatelet agent, timing and route of administration in urgent/emergent neuroendovascular stenting. Analysis of patient outcomes related to antiplatelet treatment strategy is currently ongoing.
\end{abstract}

\title{
Methods
}

This was a multicenter retrospective study evaluating antiplatelet treatment strategies in urgent/emergent neuroendovascular stenting. Data were collected related to patient demographics, procedure/stenting details, antiplatelet agent, route of administration, platelet function testing and timing in relation to stent placement.

\section{Results}

Eighty patients were included from 7 centers. Fifty-five patients were treated for ischemic stroke or high risk of stroke occurrence, 21 patients were treated for aneurysm repair (17 ruptured, 3 un-ruptured) and four patients were treated for artery dissections. Sixty (75\%) patients had a prophylactic antiplatelet agent administered prior to or during the stenting procedure. Considerable variability based on treatment center exists in the preliminary analysis suggesting some centers rely on IV antiplatelet agents whereas others consistently use oral options.

\section{Conclusions}

There is significant variability in the choice of antiplatelet agent, timing and route of administration utilized during urgent/emergent neuroendovascular stenting. 


\section{Poster 230}

\section{Road to Zero: Reduction of CAUTI in Neurocritical Patients}

Caitlin Harley, Giuliana Labella, Aria Mahtabfar, Syed Omar Shah, Marie Wilson, Maryanne McCarrin, Dennis Calguri

Jefferson Health: Jefferson Hospital of Neuroscience, Nursing Department, Philadelphia, PA, United States

\section{Background \& Purpose}

Prolonged urinary catheterization of neurocritical care patients increase risk for infection, contribute to mortality and attribute to an increased length of stay. Catheter associated urinary tract infections (CAUTIs) can be prevented but are often challenging to manage.

\section{Methods}

Improvement in CAUTI within a 40 bed neurocritical care unit began with an assessment of the problem and the development of a tiered intervention process. In 2014, a guideline for patients experiencing urinary retention after indwelling urinary catheter (IUC) removal was created, allowing clinical nurses to manage urinary retention autonomously. In 2015, a culturing protocol was assembled to give clinical decision support to the care team when patients became febrile. The protocol focused on the suspected cause and limited the amount of laboratory studies obtained. In 2017, chlorhexidine wipes were introduced as a perineal cleaning agent that could be used in daily catheter maintenance. In 2018 , an externalized female urinary catheter was introduced to monitor urinary output for incontinent patients. In 2019, the IUC ordering process was revised to provide clinical decision support for providers, requiring a reason for close monitoring of output (i.e.: hemodynamic instability, etc.)

\section{Results}

A significant decrease in CAUTI was noted. In 2014, at the beginning of the interventions, there were 45 diagnosed CAUTIs within the neurocritical care unit. In 2020, after all interventions were introduced, there were 3 diagnosed CAUTIs, noting a 93\% reduction.

\section{Conclusions}

Changes in maintenance, limiting use of IUCS \& improving clinical decision support attributed to a decrease in CAUTI with the neurocritical care unit. Future improvements could examine the necessity for IUCs in general. With the advancement clinical devices to monitor fluid status, the healthcare system may one day move to IUC-free patient care. 


\title{
Poster 231
}

The Use of Point of Care Compression Ultrasound as a Screening Tool for Lower Extremity Deep Vein Thrombosis in the Neurocritical Care Unit

\author{
Ivan Jose Cuesta Isabel, Rahul Damani, Eric Bershad, Chethan P. Venkatasubba Rao, Mohammad \\ Hirzallah, Aaron Desai, Khawja Siddiqui, Mohammad Ubaid Hafeez, Barbara L. Gutierrez-Flores
}

Baylor College of Medicine, Department of Neurology, Section of Vascular Neurology and Neurocritical, Houston, TX, United States

\section{Background \& Purpose}

Venous Thromboembolism is an important cause of morbidity and mortality in the Intensive Care Unit (ICU) and it is often asymptomatic. Surveillance of asymptomatic Neurocritical Care Unit (NCCU) patients may provide a preventive benefit. The routine use of Venous Doppler scans can be resource intensive. Two-Point Compression Ultrasound (2-CUS) has been demonstrated to be an accurate diagnostic tool in symptomatic patients, but it has not been used as a surveillance tool in the asymptomatic ICU patient. In this prospective study we evaluate the use of 2-CUS as a surveillance for asymptomatic patients in the NCCU.

\section{Methods}

This is a prospective cohort study in patients admitted to the NCCU at Baylor College of Medicine between the months of August 2020 and February 2021. A 2-CUS was performed by training neurocritical care fellows, evaluating the bilateral proximal femoral veins and popliteal veins without color doppler, on admission and every 3rd day. A formal lower extremity (LE) doppler was performed in patients with a positive 2-CUS and all patients every 7 days of NCCU admission. Inclusion criteria were patients admitted to the Neurocritical Care Service and age $>18$ years. Exclusion criteria were expected length of stay $<48$ hours, and positive test for COVID-19.

\section{Results}

In the study period, 298 patients were admitted to the NCCU. 66 patients underwent formal LE doppler of which 8 were positive. The incidence of LE in patients with a length of stay $>7$ days was $10.6 \%$. POCCUS was done in 256 patients, 9 patients were positive, 6 of which were confirmed by LE Doppler and 3 were false positives (positive predictive value $=66.7 \%$ ). Two patients had false negative 2 -CUS (negative predictive value $=95.6 \%)$. When compared to LE doppler, the sensitivity of POC-CUS was $75 \%(6 / 8)$ and specificity of $95 \%$ (57/60). The two DVT's missed on 2-CUS were located in the posterior tibial veins, which is considered distal. The sensitivity for proximal DVT (femoral and popliteal) was $100 \%$.

\section{Conclusions}

This is the first prospective study to evaluate the use of 2-CUS as a surveillance for DVT. An incidence of $10.6 \%$ was found on patients with a length of stay $>7$ days. With a sensitivity of $100 \%$ for proximal DVT and a specificity of $95 \%$, the authors conclude that 2-CUS is a valid and cost-effective tool for the surveillance of DVT in the asymptomatic NCCU patients. 


\title{
Poster 232
}

\section{Time is (a Developing) Brain: Safely Reducing Time to Hypertonic Saline Administration in a Pediatric Intensive Care Unit}

\author{
Jeffrey Moss, Nathan Chang, May Casazza, Shabnam Gaskari, Melanie Chan, Amelia Sperber, Lindsey \\ Rasmussen
}

Lucile Packard Children's Hospital, Department of Pharmacy, Palo Alto, CA, United States

\begin{abstract}
Background \& Purpose
Hypertonic sodium chloride is the first line agent for management of acute intracranial hypertension in pediatric patients. Guidelines for pediatric traumatic brain injury recommend administration of hypertonic saline 3\% (HTS) when intracranial pressure is greater than $20 \mathrm{~mm} \mathrm{Hg}$ for greater than 5 minutes. Delays in administration for pediatric patients may be multifactorial and include medication availability, weight-based dosing, commercially available dosage forms and IV access. HTS is considered a "High Alert" medication by the Institute of Safe Medication Practice and has been implicated in serious medication errors. We aimed to reduce the time to administration of HTS without increasing risk of mediation errors.
\end{abstract}

\section{Methods}

We conducted a retrospective chart review of patients in the pediatric intensive care unit (PICU) who received HTS with a frequency of "once" between $1 / 1 / 16$ and 4/30/21. HTS was added to our PICU automated dispensing cabinets (ADC) in August, 2018. Safety measures included alerts at the point of dispensing and "high alert" auxiliary stickers. Additional interventions since 2018 include the creation of a pediatric neurocritical care team, development of an ICP management order set including HTS in a tiered treatment approach, targeted intervention for prescribers, nurses and pharmacists and prioritizing orders for HTS to have a default priority of "STAT."

\section{Results}

1372 doses of HTS were administered between $1 / 1 / 16$ and 4/30/21. The median time from HTS order to administration was decreased from a baseline of 43 minutes to 33 minutes after addition of HTC to PICU ADCs. There have been no documented medication errors related to HTS.

\section{Conclusions}

Interventions including stocking of HTS in PICU ADCs may improve time to administration of HTS in pediatric neurologic emergencies. Safeguard may decrease the risk of medication errors. Additional studies are needed to demonstrate the correlation with time to administration and clinical outcomes. 
Poster 233

\title{
A Cloud-based Platform for Data Management in Traumatic Brain Injury Clinical Trials
}

\author{
J. Stover, C. Maddux, ES Rosenthal, B. Foreman, A. Rodriguez, D. Moberg
}

School of Biomedical Engineering, Science and Health Systems, Drexel University, Philadelphia PA, United States

\section{Background \& Purpose}

Due to the complexity of traumatic brain injury (TBI), related clinical trials must collect high-resolution multimodal data (MMD). Problematic aspects of this include time synchronization of interventions, aggregation of metadata, and creation of a common repository. We aim to overcome these problems by creating a cloud-based system (CONNECT) to manage a common MMD repository for precision management of TBI and discovery of trends in neuro-worsening. We partnered with several TBI trials to identify key features of CONNECT.

\section{Methods}

Clinical trials upload physiologic data from study subjects with TBI using CONNECT. Data are catalogued and labelled with metadata that is stored in a Structured Query Language database. Medication and intervention data are time-synchronized and mapped to standard column names. All data are converted into human-readable files that use the Hierarchical Data Format 5 (HDF5) format. Finally, all data are archived on cloud storage. CONNECT can query uploaded data for quality assurance, visualization, and analysis.

\section{Results}

The clinical trial TRACK-TBI managed MMD archives $(n=258)$ of 124 subjects with CONNECT. 258/258 (100\%) were uploaded and 257/258 (99.6\%) were converted to HDF5. One site provided medication data for 17 subjects; 17/17 (100\%) were uploaded. 124/124 (100\%) subjects' metadata were extracted. The size of the repository was 1.23 TB; 741.52 GB was HDF5 data. Currently, BOOST-3 is also utilizing CONNECT to manage MMD for their study, which is projected to enrol 1096 subjects. CONNECT will also manage data for PRECICECAP and University of Pennsylvania.

\section{Conclusions}

CONNECT successfully fulfils MMD management requirements in several clinical trials. Future work will include development of CONNECT for precision management of TBI and discovery of trends in neuroworsening and validation in a variety of neurocritical care settings using short term endpoints. Pathways for use in austere environments or prolonged care will also be investigated 


\section{Poster 234}

\section{Could Time Of Mechanical Ventilation Affect Intracranial Compliance In COVID-19 Patients?}

Gisele S Silva, Ana Flávia Silveira, Nelci Zanon Collange, Cintya Yukie Hayashi, Samantha Longhi Simões de Almeida, João Brainer Clares de Andrade, 6. Salómon Rojas, Fabiano Moulin de Moraes, Gustavo Frigieri, Thiago Luiz de Russo

Federal University of São Paulo - Department of Neurology, São Paulo/SP, Brazil

\section{Background \& Purpose}

Background: Ventilatory techniques used to treat hypoxemia caused by COVID-19 can cause an increase of blood $\mathrm{CO} 2$ levels that could generate vasodilatation of brain vessels and, thus, changes in intracranial compliance (ICC). Aim: To verify whether time of mechanical ventilation can affect ICC in patients with COVID-19.

\section{Methods}

Methods: COVID-19 patients were enrolled in a multicenter observational study at three hospitals in São Paulo/Brazil from June to October 2020. Both genders, $\geq 18$ years-old, admitted to an intensive care unit $\leq 72$ hours and intubated $\leq 24$ hours due to COVID-19, symptoms onset $<15$ days and no acute neurological symptoms at presentation were included. Clinical and epidemiological information (age, sex, comorbidities, and neurological symptoms) were assessed from medical reports. The intracranial pressure (ICP) morphology was non-invasively assessed by a device (Brain4Care Inc, FDA approved) positioned in the temporal bone. The ICP signal provides three distinct peaks (P1, 2, and 3). The reason P2/P1 reflects ICC changes. Daily monitoring occurred during seven consecutive days for 60 minutes/daily. Descriptive analyses were performed for sample characterization, while repeated measures one-way ANOVA (with $\mathrm{p}<0.05$ as significant) compared P2/P1 on 1st, 3rd, and 5th days of intubation.

\section{Results}

Results: Eleven patients (73\% man, mean age of $64 \pm 11$ years-old) were evaluated. Diabetes mellitus and systemic arterial hypertension were the most common comorbidities (64\% each), and $36 \%$ presented neurological symptoms due to COVID-19. The P2/P1 mean was $1.07 \pm 0.29,1.10 \pm 0.26$, and $1.02 \pm 0.18$ on the first, third, and fifth day, respectively, with no differences overtime $(p=0.73)$.

\section{Conclusions}

Conclusion: Time of mechanical ventilation did not affect ICC in COVID-19 patients suggesting that even aggressive and prolonged mechanical ventilation can be performed safely from a brain perspective if adequate monitoring is in place. Our results are preliminary and must be interpreted with caution. 
Poster 235

\section{Detection of Cerebral Edema Post-traumatic Brain Injury using Non-invasive Cranial Accelerometry}

Jonathan Shih, Maximilian Vuong, Rachel Muster, Dominica Randazzo, Edilberto Amorim, Wade Smith

University of California, San Francisco, Department of Neurology, San Francisco, CA, United States

\section{Background \& Purpose}

Cardiac forces on the skull and brain produce the "headpulse." The headpulse is abnormal in patients who have suffered acute ischemic stroke, vasospasm following aneurysmal subarachnoid hemorrhage, and concussion. Following concussion, spectral components of the headpulse rise then return to normal weeks later. Here, we measure the headpulse in patients with traumatic brain injury (TBI) to determine if the same frequency shift is seen in more severe forms of brain injury.

\section{Methods}

The headpulse was measured using bilateral three-axis accelerometers affixed to a headset placed over the temporal bone along with three-lead electrocardiography (ECG). Five-minute recordings were performed on seated subjects with TBI and evidence of focal cerebral edema on computed tomography. Fourier transforms of each recording were computed and average heartrate determined from the ECG. Spectral amplitude at the first through ninth harmonic of the heartrate were measured and normalized to sum of these amplitudes. R1 was defined as the fifth harmonic divided by the first harmonic, R2 as the average of the seventh and eight harmonics divided by the first harmonic. This methodology was chosen to mirror the algorithm used in prior work with concussion. P-values were derived using twotailed, unequal variances t-tests.

\section{Results}

The frequency domain of the headpulse was compared in 10 TBI subjects and 10 controls without brain injury or known intracranial abnormality. Analysis revealed a spectral shift towards higher harmonics in TBI subjects. Both R1 and R2 values were significantly higher in TBI subjects compared to controls $(p<0.05$ in the vertical, anterior-posterior, and lateral axes).

\section{Conclusions}

Headpulse frequency spectra is abnormal in TBI subjects with cerebral edema compared to controls in a fashion highly similar to concussion. This significant frequency shift may establish a new diagnostic test for all forms of TBI. We are now exploring the progression of headpulse frequency shifts in long-term follow-up subjects. 


\section{Poster 236}

\section{Effect of Early Passive Exercise of Varying Intensities on Cerebral Autoregulation in Acutely Brain Injured Patients: A Descriptive Pilot Study}

Madihah Hepburn, Catherine Hassett, Amin Moien, Alexandra Harrison, Patrick Cetina, Joao Gomes

Cerebrovascular Center, Cleveland Clinic, Cleveland, $\mathrm{OH}$, United States

\section{Background \& Purpose}

Lower extremity ergometry(LER) is effective for passive exercise in immobilized patients. Previous studies used intermittent monitoring to determine the effect of LER-activity on cerebral autoregulation(CAR). Effects of early mobility and exercise intensity on CAR in brain injured patients have not been described. Our study describes the effect of passive cycling on brain injured patients admitted to the neurological intensive care unit (NICU). Using continuous monitoring of blood pressure, heart rate and intracranial pressure(ICP) with transcranial doppler(TCD) monitoring of the injured hemisphere to calculate changes in CAR during exercise.

\section{Methods}

Descriptive study of patients with acute brain injury, monitored during early passive LER-activity. Continuous hemodynamic and ICP monitoring recorded during LER-activity. The Multigon TCD Robotic Headband System used to acquire mean flow velocities (MFV) from the MCA ipsilateral to the injured cerebral hemisphere during passive LER-activity at varying intensities for 30-minutes. Continuous variables(hemodynamics, MFV, ICP) recorded with ICM+ software. ICM+software used to calculate coefficient variables of cerebrovascular pressure reactivity(PRx) and a moving correlation co-efficient(Mxa) as continuous indices of cerebral autoregulation.

\section{Results}

Descriptive analysis of 8-brain injured(intracranial hemorrhage, ischemic stroke, subarachnoid hemorrhage) patients during early ICU admission. Prx /Mxa calculations calculated demonstrated 4/8patients had intact CAR initially but as LER-activity intensified CAR became impaired. CAR impairment persisted in 2/8-patients during the 10-minute post-exercise period.

\section{Conclusions}

Intensive passive activity in the early phase of brain injury may worsen CAR in acutely brain injured patients. Sample size was too small to correlate Prx /Mxa to find individual CAR thresholds for varying brain injuries. Simple hemodynamic and ICP monitoring may not capture scope of potential brain injury, since patients with baseline intact CAR demonstrated autoregulatory changes as exercise intensified. Early mobility in the ICU can be beneficial but further prospective studies are needed to determine best timing and intensity of activity in brain injured patients. 


\section{Poster 237}

\section{Extracting Meaning from Neurocritical Care Annotations Requires a Brain Injury-Specific Natural Language Processing Vocabulary}

Eric S. Rosenthal, Guneeti Sharma, DaiWai M. Olson, Sophie Ack, India A. Lissak, Craig Maddux, Edilberto Amorim, J. Randall Moorman, Xiao Hu, Gilles Clermont, Dick Moberg, Brandon Foreman

Massachusetts General Hospital, Boston, MA, United States

\section{Background \& Purpose}

To model the neurocritical patient care environment, we sought to extract meaning from text annotations documented during multimodality neuromonitoring.

\section{Methods}

Using exhaustive expert labelling, we mapped neuromonitoring annotations from a single-center neurocritical care unit to the six SNOMED-CT concept domains: Drug, Observation, Condition, Procedure, Measurement, Device. We tested a standardized SNOMED-CT vocabulary for accuracy predicting expert labels. We then trained a long short-term memory (LSTM) model on $70 \%$ of the unique labels, using class membership from unsupervised KMeans clustering and unigram tokens from a standard library. Unigram clustering and word2vec features in the input file aimed to improve accuracy compared to a traditional TF-IDF approach for contextualized word text, overcoming limitations of unidentified, misspelled, abbreviated, and jargon terms. The novel model was then tested on the $30 \%$ hold-out set.

\section{Results}

16,374 annotations comprising 3,582 unique annotations were available. The existing SNOMED-CT vocabulary yielded $26.42 \%$ accuracy for classifying annotations compared to expert annotation. Ten clusters appeared optimal, inspecting a sum-of-squares error function. Dimensionality reduction using principal component analysis and linear discriminant analysis assisted interpretation. Specifically, the 10 clusters generated for the novel model included unigram terms with intuitive class membership; the top 15 terms in each cluster were over-represented with salience for specific topics (clusters \#1 and \#3, cerebral metabolism; \#2, hemodynamics; \#4 and \#10, ventilation; \#5, cerebrospinal fluid management; \#6, sedation; \#7, catheter management; \#8, electroencephalography; and \#9, intracranial monitors). The novel model predicted SNOMED-CT concept domain labels with $85.35 \%$ training and $82.36 \%$ testing accuracy.

\section{Conclusions}

The standard SNOMED-CT vocabulary poorly classified neurocritical care annotations into SNOMED-CT concept domains. A novel hybrid model representing a brain-injury-specific vocabulary demonstrated high testing accuracy utilizing expert labels and unsupervised clustering to predict the correct SNOMEDCT concept domain. Future directions include topic modeling within SNOMED-CT concept domains, optimizing classification optimization using Latent Dirichlet Allocation, and external validation. 


\section{Poster 238}

\section{Factors Associated with Brain Tissue Oxygenation Changes after Red Blood Cell Transfusion in Acute Brain Injury Patients}

Elisa Gouvêa Bogossian, Verena Rass, Anna Lindner, Carolina laquianello, John Paul Miroz, Elaine Cavalcante Dos Santos, Hassane Njimi, Jacques Creteur, Mauro Oddo, Raimund Helbok, Fabio Silvio Taccone

Université Libre de Bruxelles - Erasme Hospital, Brussels, Belgium

\section{Background \& Purpose}

Background: Anemia is common after acute brain injury and can be associated with brain tissue hypoxia. Red blood cell transfusion (RBCT) can improve brain oxygenation; however, predictors of such improvement remain unknown.

\section{Methods}

This multicentric retrospective cohort study (2012-2020) included all patients admitted to 3 European Intensive Care Units with acute brain injury who were monitored with brain tissue oxygenation (PbtO2) catheters and received at least one RBCT. We aimed to identify the factors associated with PbtO2 increase ( $>20 \%$ from baseline value) after RBCT, using a generalized mixed model (GMM).

\section{Results}

We included 69 patients receiving a total of 109 RBCTs after a median of 9 [5-13 days] days after injury. Baseline hemoglobin $(\mathrm{Hb})$ and PbtO2 were 7.9 [7.3-8.7] g/dL and 25 [20-30] mmHg, respectively; 2 hours after RBCT, the median absolute $\mathrm{Hb}$ and $\mathrm{PbtO} 2$ increases from baseline were 1.2 [0.8-1.8] g/dL ( $p$ $=0.001)$ and $3[0-6] \mathrm{mmHg}(p=0.001)$. A 20\% increase in PbtO2 after RBCT was observed in 45 (41\%) transfusions. Subarachnoid hemorrhage $(\mathrm{SAH})$ as underlying disease, high heart rate (HR) and low PbtO2 at baseline were independently associated with a $20 \%$ increase in PbtO2 after RBCT. Baseline PbtO2 had an area under receiver operator characteristic of 0.73 (95\% Cl 0.64-0.83) to predict PbtO2 increase; a $\mathrm{PbtO} 2$ of $20 \mathrm{mmHg}$ had a sensitivity of $58 \%$ and a specificity of $73 \%$ to predict PbtO2 increase after RBCT.

\section{Conclusions}

Lower PbtO2 values, high $\mathrm{HR}$ and $\mathrm{SAH}$ at baseline could predict a significant increase in brain oxygenation after RBCT. 


\section{Poster 239}

\section{Inter- and Intra-Rater Reliability of Optic Nerve Sheath Diameter (ONSD) Ultrasound Performed According To The CLOSED-Protocol}

Jakob Pansell, Max Bell, Peter Rudberg, Ola Friman, Charith Cooray

Karolinska Institute, Institution of Clinical Neuroscience, Stockholm, Sweden

\section{Background \& Purpose}

Treatment of elevated intracranial pressure (ICP) is central to neurocritical care, but not all patients are eligible for invasive ICP-monitoring. Therefore, non-invasive estimates of ICP may benefit many patients. A promising option is ultrasound measurement of the Optic Nerve Sheath Diameter (ONSD). However, meta-analyses of ONSD cut-offs for elevated ICP show wide confidence intervals. This is likely due to varying measurement methods, baseline variations and inter-rater variability. To remedy this, the CLOSED protocol has been suggested but not yet validated. Corrections for Eyeball Diameter (ED) and Optic Nerve Diameter (OND) have also been suggested to compensate for baseline variations of ONSD. The purpose of this study is to validate a protocol based on CLOSED and to compare two different measurement methods for ONSD ultrasound.

\section{Methods}

Two operators measured ONSD, ED and OND twice in 20 patients. Measurements were performed in one short session and the operators were blinded to each other's measurements. The CLOSED-protocol was used for sonographic settings and landmarks. ONSD was measured both between the inner limits (ONSDi) and the outer limits (ONSDo) of the dura, the two methods in use. Patients were not deemed at risk of ICP fluctuations. Intra-class correlation (ICC) was calculated for inter-rater and intra-rater reliability.

\section{Results}

ICC for inter-rater reliability of ONSDo and ONSDi $(95 \% \mathrm{Cl})$ were $0.96(0.93,0.98)$ and $0.88(0.79,0.94)$. ICC for intra-rater reliability of ONSDo and ONSDi $(95 \% \mathrm{Cl})$ were $0.94(0.97,0.99)$ and $0.93(0.87,0.96)$. All corrected measurements yielded ICC:S $>0.75$. There was no significant bias between operators.

\section{Conclusions}

This protocol, based on CLOSED, yields better ICC for inter- and intra-rater reliability of ONSD ultrasound compared to previous studies. ONSD-corrections for ED and OND can be performed with a good to excellent inter-rater reliability. The optimal ONSD parameter for estimating ICP remains unanswered. 


\title{
Poster 240
}

\section{Intracranial Compliance and Cerebral Hemodynamic Disorders in Severe COVID-19}

\author{
Sérgio Brasil, Fabio Taccone
}

University of São Paulo, São Paulo, Brazil

\section{Background \& Purpose}

One of the possible mechanisms by which the new coronavirus (SARS-Cov2) could induce brain damage is the production of cerebral hemodynamics disorders (CHD) and intracranial compliance impairment (ICCI) due to the elevation of intracranial pressure (ICP), especially in cases of severe acute respiratory syndrome (SARS). The present study was dedicated to identifying CHD and ICCI, to evaluate their association with short-term clinical outcomes in SARS for COVID-19.

\section{Methods}

50 consecutive critically ill COVID-19 patients were studied with a noninvasive technique (B4C device) that reproduces ICP waveforms and transcranial Doppler (TCD). Subjects were included upon ICU admission; CHD were documented by the assessment of flow velocities in the middle cerebral arteries (mCBFV), pulsatility index (PI) and estimated cerebral perfusion pressure (eCPP) whereas ICCI was assessed by using the P2/P1 ratio of estimated ICP curve. The primary composite outcome was unsuccessful weaning from respiratory support or death up to seven days after last TCD and B4C assessments.

\section{Results}

At ICU admission ( $n=50)$, only P2/P1 (1.20 [1.00-1.28] vs. 1.00 [0.88-1.16]; $p=0.03$ ) and elCP (14 [11-25] vs. 11 [7-15] mmHg; $p=0.01$ ) were significantly higher among patients with unfavorable outcome (UO) than others. Patients with UO had a significantly higher CVH/ICC score (9 [8-12] vs. 6 [5-7]; $p<0.001)$ than those with favorable outcome; the area under the receiver operating curve (AUROC) for CVH/ICC score to predict UO was 0.86 (95\% Cls 0.75-0.97); a score $>8.5$ had 63 (46-77)\% sensitivity and 87 (6297)\% specificity to predict UO. For those patients undergoing a second assessment $(n=29)$ after a median of 11 (5-31) days, all measured variables were similar between the two time-points. No differences in the measured variables between ICU non-survivors $(n=30)$ and survivors were observed.

\section{Conclusions}

ICCI and CHD are often present in COVID-19 severe illness and could accurately predict early poor outcome. 


\title{
Poster 241
}

\section{Intracranial Pressure is More than a Number: Lessons from the Liver Intensive Care Unit}

Catherine E. Hassett, Madihah Hepburn, Moein Amin, Christina Lindenmeyer, Aanchal Kapoor, Joao Gomes

\author{
Cleveland Clinic, Cerebrovascular Center, Cleveland, $\mathrm{OH}$, United States
}

\section{Background \& Purpose}

Cerebral edema is a significant cause of death in acute liver failure (ALF) patients. Invasive monitoring of intracranial pressure (ICP) can be used to guide management. There is limited understanding of cerebral autoregulation (CA) alterations during ALF. We report our preliminary experience.

\section{Methods}

Baseline demographics, clinical information, and multimodality neurologic monitoring data in ALF patients with Grade 4 hepatic encephalopathy $(\mathrm{HE})$ were retrospectively analyzed using ICM+ software (Cambridge, UK). Pressure Reactivity Index (PRx), a model-based index of dynamic CA, was calculated for the duration for the recording and considered impaired above the previously validated target of 0.3 . Data is presented as range or a mean with standard deviations.

\section{Results}

4 patients ( $75 \%$ female, mean $39 \pm 20$ years old) with idiopathic ALF and Grade 4 HE were listed for liver transplantation. On day of monitoring, all patients had a glasgow coma scale of 3T. Per protocol, continuous infusion of $3 \%$ hypertonic saline was used for sodium goal of $145-155 \mathrm{mmol} / \mathrm{L}$ and vasopressors for a targeted mean arterial pressure of $>65 \mathrm{mmHg}$. At time of monitoring, mean sodium $142 \pm 3.98 \mathrm{mmol} / \mathrm{L}$, ammonia $249 \pm 135 \mathrm{umol} / \mathrm{L}$, and model for end liver stage disease (MELD) score 26.5 \pm 4.5 were noted. During monitoring, ICP $(6.23 \pm 6.94 \mathrm{mmHg})$ remained within normal limits except for one episode of ICP crisis requiring acute hyperosmolar therapy. Despite normal ICP, mean PRx (0.43 \pm 0.231 ) was found to be impaired in 3 of the 4 patients. All patients spent at least $50 \%$ of the time monitored within the impaired CA range. Only one patient survived the ALF period.

\section{Conclusions}

Despite normal ICP values, CA appears to be impaired in the severe ALF patient. The clinical significance of this finding, especially its impact on ALF patient outcomes, needs to be further defined in prospective studies. 


\title{
Poster 242
}

\section{Replay of Learned Neural Sequences in Human Motor Cortex Observed Using an Intracortical Brain Computer Interface}

\author{
Daniel B. Rubin, Tommy Hosman, Jessica N. Kelemen, Anastasia Kapitonava, Francis R. Willett, Brian F. \\ Coughlin, Eyal Y. Kimchi, John D. Simeral, Leigh R. Hochberg, Sydney S. Cash \\ Massachusetts General Hospital, Department of Neurology, Boston, MA, United States
}

\begin{abstract}
Background \& Purpose
Brain-computer interfaces have the potential to restore communicative function after severe neurologic injury. Our ability to decode high-dimensional patterns of neural activity underlying complex communicative behavior will benefit from understanding how cortex stores and executes motor sequence commands. One proposed mechanism is through repeated offline "replay" of neural activity during sleep. Though amply demonstrated in rodent hippocampus, replay is less well documented in humans, particularly in motor cortex. To address this knowledge gap, we recorded from human motor cortex as a research participant performed a novel motor task and subsequently slept overnight.
\end{abstract}

\section{Methods}

A 36-year-old man with tetraplegia secondary to cervical spinal cord injury enrolled in the BrainGate brain-computer interface clinical trial had two 96-channel microelectrode arrays placed chronically into left pre-central gyrus (PCG). Single- and multi-unit activity was recorded while he played a color/sound matching memory game. On each of 160 trials, he was cued to four screen locations; his task was to move a computer cursor to those locations in the correct sequence. Intended movements were decoded from neuronal activity via a real-time steady-state Kalman filter allowing the participant neural cursor control. Intracortical PCG activity and scalp EEG were also recorded overnight as he slept.

\section{Results}

When decoded using the same steady-state Kalman filter parameters, intracortical neural signals recorded overnight replayed the target sequence from the memory game throughout sleep. Replay occurred at speeds ranging from one to four times the initial task execution and was most prevalent during slow-wave sleep.

\section{Conclusions}

Recent visuomotor skill acquisition in humans is accompanied by replay of the corresponding motor cortex neural activity during sleep. These findings provide insight into cortical mechanisms of motor sequence learning that will facilitate the development of improved decoding algorithms for braincomputer interfaces aimed at restoring communication and functional independence after severe neurologic injury. 


\title{
Poster 243
}

\section{Targeted Temperature Management for Patients with Cardiac Arrest and High Bleeding Risk}

\author{
Melinda Miller, Kim Fowler, Farid Sadaka
}

Mercy Hospital St Louis/Department of Critical Care Medicine, St Louis, MO, United States

\section{Background \& Purpose}

Targeted Temperature Management (TTM) improves survival and neurologic outcome and is recommended for cardiac arrest (CA) survivors by international guidelines. Major studies have excluded trauma patient (TP), postoperative patients (POP), and patients with GI bleed (GIB) mainly because of high risk of bleeding. We sought to determine whether TTM is feasible and safe in this patient population.

\section{Methods}

A retrospective study was conducted at a single Level I Trauma Center reviewing adult patients presenting as a traumatic arrest or cardiac arrest in the postoperative period or from GIB with a Glasgow Coma Scale $<8$ after return of circulation who were treated with TTM. Data on these patients were collected from the APACHE Outcome database and medical records. Baseline characteristics included age, gender, APACHE III scores, acute physiologic scores (APS), shockable vs nonshockable (NS) rhythm, and goal temperature ( $330 \mathrm{C}$ vs $360 \mathrm{C}$ for 24 hours). Outcome was survival to hospital discharge with good neurologic outcome. Bleeding complication was defined as need for transfusion after initiation of TTM and up to 24 hours after discontinuation of the cooling therapy.

\section{Results}

A total of 10 patients were included ( $4 \mathrm{~T}, 3 \mathrm{POP}$, and $3 \mathrm{GIB}$ ) with average age ( $55+/-17$ years), gender (60\% male), APACHE III (93 +/- 21), APS ( $86+/-23)$, NS rhythm (90\%), and temperature goal ( $40 \%$ at $330 \mathrm{C}, 60 \%$ at $360 \mathrm{C})$. Survival with good neurologic outcome at discharge was $40 \%$. One patient (10\%) developed bleeding complication requiring transfusion of 1 unit RBC.

\section{Conclusions}

Trauma patient, postoperative patients, and patients with GI bleed with cardiac arrest previously excluded from TTM studies appear to have comparable outcomes and acceptable incidence of bleeding complication compared with standard TTM patients. These findings should be further investigated in prospective studies. 


\title{
Poster 244
}

\section{The Neurological Injury Motion Sensing (NIMS) Project: Quantifying, Decoding, and Validating Accelerometry Features in the Neurocritical Care Setting}

\author{
Shubhayu Bhattacharyay, Matthew Wang, Peter Dziedzic, Eusebia Calvillo, Han B. Kim, Eshan Joshi, John \\ Rattray, Ralph Etienne-Cummings, Pawel Kudela, Robert D. Stevens \\ Laboratory of Computational Intensive Care Medicine, Johns Hopkins University, Baltimore, MD, United \\ States
}

\section{Background \& Purpose}

Motor activity is an important phenotypic variable which is difficult to quantify in the clinical setting. Here, we tested a novel accelerometry-based system to continuously monitor motion in the neurosciences critical care unit (NCCU).

\section{Methods}

Patients admitted to the NCCU with severe brain injury (SBI) were prospectively enrolled $(n=69)$. Six accelerometers were placed on elbows, wrists, and ankles, sampling data at $10 \mathrm{~Hz}$. We extracted 6 types of features ( 2 time-, 3 frequency-, 1 wavelet-domain) from non-overlapping 5 -second windows of filtered accelerometry data. Motion features across extremity sensors were binned in 4 observation windows $(0.5,1,3$, and 6 hours) directly preceding nurse-assessed Glasgow Coma Scale (GCS) evaluations $(n=653$ ). Dimensionality reduction and feature importance ranking was performed using linear optimal low-rank (LOL) projection, with motor sub-score of GCS (GCSm) as the outcome label. Embedded features were then used in a multiclass logistic regression models to detect GCSm.

\section{Results}

Sixty-nine patients with SBI underwent continuous motion sensing (median duration, $24 \mathrm{~h}$ ). Percentages of daily activity were significantly correlated with GCSm (Spearman's coefficient $=0.25, p=0.04$ ). Upper extremity features were more important than ipsilateral lower extremity-based features $(p<0.0001)$, and frequency-domain entropy (FDE) and signal magnitude area (SMA), particularly of the right wrist, were the most important features in explaining the variance of GCSm. Model discrimination varied depending on the clinical label: AUROC > 0.80 for GCSm 1-4, AUROC > 0.70 for GCSm 6, and AUROC > 0.60 for GCSm 5. Model performance improved significantly as the observation window increased from 1 to $6 \mathrm{~h}$.

\section{Conclusions}

Models using continuous accelerometry features accurately discriminated low and high levels of clinically defined movement. The approach establishes the feasibility of an automated system of quantitative motion assessment in the NCCU which could improve individualized treatment and prognosis for SBI patients. 
Poster 245

\section{Ultra-Long cEEG Recording Using Subgaleal Electrode Arrays in Critically III Patients with COVID-19}

Laith Altaweel, Danielle Dang, Jing Wang, Shashank Sinha, Mehul Desai, Hussain Dhanani, Sairah Bashir, Asma Zakaria, Bahiru Zelalam, Pouya Tahsili Fahadan, Mohan Kurukumbi, Erik Osborn, Allen Waziri

Departments of Medicine and Neurosciences, Inova, Falls Church, VA, United States

\section{Background \& Purpose}

cEEG is indicated in critically-ill COVID-19 patients for seizure monitoring, detection of intracranial events and management of sedation. Given the need to minimize contact of technical personnel with this patient population, we explored the utility of bedside subgaleal (SG) electrode insertion for longterm cEEG monitoring.

\section{Methods}

Sixteen critically-ill COVID-19 patients were included; all were mechanically ventilated and fourteen were undergoing ECMO. All patients were receiving therapeutic anticoagulation at the time of device insertion. Bihemispheric parasagittal electrodes with eight recording channels were placed by attending ICU staff using a trocar-and-sheath device. Minor bleeding at entry/exit points related to anticoagulation in several patients was easily controlled through pressure or a single U-stitch. Continuous impedance data for each recording contact was collected throughout the monitoring period. Raw and quantitative SGEEG data were assessed on a regular basis by attending neurocritical care staff.

\section{Results}

There were no adverse events associated with device insertion or long-term SGEEG monitoring. Bihemispheric SGEEG was successfully recorded in all patients; mean length of continuous recording was $11.3+/-9.0$ days with the longest continuous recording at 32.7 days. Evaluation of continuous data across all subjects demonstrated impedance of less than $10 \mathrm{kOhm}$ for all contacts through $85.7 \%$ of the aggregate monitoring period; at least one bipolar channel was available for each hemisphere for $93.7 \%$ of the aggregate monitoring period. Electrographic seizures were detected by SGEEG and treated in three out of sixteen patients (18.8\%). SGEEG-derived burst-suppression data were successfully used to guide sedation management. No catastrophic intracranial events were experienced within this small cohort.

\section{Conclusions}

SGEEG offers the potential for long-term, high-fidelity cEEG recording without the need for continuous technical maintenance. The attractive signal quality and durability of this technique may hold promise for continuous neuromonitoring across the broader spectrum of ICU care. 


\section{Poster 246}

\section{Use of a Novel Self-irrigating Ventricular Catheter Drainage System: A Case Series}

Christa O’Hana Nobleza, Zachary S. Smalley, Jason Tullis, Gustavo Luzardo

University of Mississippi Medical Center, Jackson, FL, United States

\section{Background \& Purpose}

Irrigating ventricular drainage systems are a recent technological development that are theoretically thought to help reduce occlusion rates, thereby reducing the need for flushing and subsequent hemorrhagic and infectious complications. There is also hope they can hasten hemorrhage clearance time, thereby reducing the duration of need for ventriculostomy and therefore hospital length of stay. We report our experience with the use of this novel self-irrigating ventricular drainage system.

\section{Methods}

We present a case series of patients that received ventriculostomy with the IRRAFLOW'M device. Demographic data including age, gender, past medical history, indications for ventriculostomy insertion, complications, duration of drainage and management are presented.

\section{Results}

We used these catheters in 3 patients from mid 2019 through January 2020. This ventricular drainage system was used for intraventricular hemorrhage, hydrocephalus associated with brain abscess and for ventriculitis. Safety concerns were associated with catheter position and port location.

\section{Conclusions}

Our experience showed several neuropathologies that IRRAFLOW ${ }^{\mathrm{TM}}$ can be used for. We also presented potential safety issues and potential mitigation strategies for these. Further prospective studies should be done to assess the benefit and cost-effectiveness of IRRAFLOW ${ }^{\mathrm{TM}}$. 
Poster 247

\section{Validation of a Novel Non-invasive ICP Monitor and Correlation with Clinical Outcome}

Rohan Mathur, Eusebia Calvillo, Peter Dziedzik, Jose I. Suarez, Robert Stevens

Division of Neurocritical Care, The Johns Hopkins University School of Medicine, Baltimore, MD, United States

\section{Background \& Purpose}

Detection and management of ICP often relies either on clinical examinations, that may be ambiguous or unreliable in detecting early changes, or on highly-invasive monitors such as intraventricular catheters that have significant associated risks and complications. The Brain4Care BcSs-PICNI-2000 ${ }^{\circledR}$ uses a non-invasive mechanical extensometer to detect subtle skull deformations, generating waveforms with similar morphology to invasive ICP waveform. In this study of patients with acute brain injury, we hypothesized that (1) a correlation exists between the P2/P1 ratio generated by the Brain4Care and invasive ICP waveform, and (2) changes in the P2/P1 ratio of the Brain4Care device would predict a decline in GCS.

\section{Methods}

Patients admitted to the ICU with different etiologies of acute brain injury were prospectively recruited, of whom 8 had intraventricular catheters. Daily 10-minute recordings of Brain4Care waveform, invasive ICP waveforms, other physiologic data and clinical exams were performed. Waveform averaging was done for every minute of recording. P1 and P2 peaks were predicted using a customized algorithm.

\section{Results}

32 recordings were made in 15 patients. A strong correlation was found (Pearson's $R=0.91$ ) between the non-invasive and invasive P2/P1 waveform ratios. ROC analysis demonstrated AUROC 0.96 , Sensitivity $90 \%$, Specificity of $83 \%$ detecting a matched P2/P1 ratio compared to invasive ICP waveforms when using a 6-point ordinal rating scale. Bland-Altman plots were analyzed and showed high agreeability between invasive and Brain4Care data. In a subset of 8 patients with P2/P1 changes over time, we found that in all but one case, an increase in P2/P1 ratio correlated with declining GCS.

\section{Conclusions}

There is a robust correlation between the morphology and P2/P1 characteristic of the non-invasive Brain4Care sensor waveform and invasive ICP waveform. Results, albeit preliminary, indicate that increases in the P2/P1 ratio predict clinically significant neurological deterioration. Research is needed to further explore and validate these findings. 
Poster 248

\section{A Retrospective Single Center Analysis Comparing the Efficacy of Caffeine/Acetaminophen/Butalbital (Fioricet ${ }^{\circledR}$ ) to Tapentadol (Nucynta ${ }^{\circledR}$ ) for Headache Management in a Neurosurgical Intensive Care Unit (NSICU)}

Seohyun Choi, Christopher Makosiej, Alison Brophy, Angela Antoniello, Kristin Fless, Nirav Mistry

Saint Barnabas Medical Center, Livingston, NJ, United States

\section{Background \& Purpose}

Headache management after neurosurgical interventions remains a challenge. NSAIDs, opioids and acetaminophen are often used; however, they are frequently sub-optimal. Therefore, at our institution, caffeine/acetaminophen/butalbital and tapentadol are commonly prescribed. The purpose of this study was to compare the efficacy of caffeine/acetaminophen/butalbital versus tapentadol for the treatment of headaches.

\section{Methods}

This study was IRB approved single center retrospective chart review and included all adult neurosurgical patients (>18 years old) between July 1, 2017 and June 30, 2019 admitted to the NSICU. Primary outcome was the percentage of patients who received opioid rescue medications in 24 hours of starting either of study medications. Descriptive statistics were utilized for baseline characteristics and secondary outcomes, and the primary endpoint was analyzed with Fisher's exact test. An alpha value of 0.05 determined the statistical significance.

\section{Results}

110 patients were included in our analysis out of 778 screened. Of 110 patients, 82 received caffeine/acetaminophen/butalbital while 28 received tapentadol. Eighteen of 82 patients (22\%) in caffeine/acetaminophen/butalbital group and 2 of 28 patients (7.1\%) in tapentadol group received at least 1 dose of opioid rescue medications $(p=0.09)$. Mean time to first dose of rescue medication was 149 minutes in caffeine/acetaminophen/butalbital group ( $0-311$ minutes) and 249 minutes in tapentadol group (180 - 318 minutes). Glasgow Coma Scale (GCS) score worsened in 2 of 82 (2.4\%) patients in caffeine/acetaminophen/butalbital group versus none in tapentadol group.

\section{Conclusions}

Tapentadol use in neurosurgical patients trended towards less requirement of rescue medications and a prolonged time to first dose of rescue medication. The results should be further evaluated to facilitate the implementation of safe and effective management of headaches in these patients. 
Poster 249

\section{D-dimer is Valuable for Predicting the Extension of Isolated Distal Deep Vein Thrombosis of the Lower Limb in Neurocritical Patients}

Rui Tang, Gao Zhi, Du Ming, Liu Haiyan, Yang Yanyan, Ji Zongsu, Zhou Ming

Neurocritical Care Unit, The First Affiliated Hospital of USTC, Division of Life Sciences and Medici, Hefei, Anhui Province, China

\section{Background \& Purpose}

Isolated distal deep vein thrombosis (IDDVT) of the lower limb and its extension to the proximal deep veins have high incidence rates in patients with acute brain injury (ABI). The objective of the study was to determine the value of dynamic changes in coagulation function in predicting the occurrence and extension of IDDVT.

\section{Methods}

In this retrospective cohort study, $\mathrm{ABI}$ patients during the perioperative period in a neurocritical care unit (NICU) of a university hospital were identified from September 2019 to May 2020. Complete compression ultrasound was used by a senior sonographer to diagnose and detect the extension of IDDVT. Coagulation function was recorded at a series of time points during the perioperative period.

\section{Results}

A total of $164 \mathrm{ABI}$ patients were identified, including 26 acute traumatic brain injury patients, 83 acute hemorrhagic stroke patients, 22 acute ischemic stroke patients and 33 acute aneurysmal subarachnoid hemorrhage patients. Most of the patients were elderly and male, and most had severe cases. The rates of IDDVT occurrence and extension were $52 \%$ and $20 \%$, respectively. Age, sex, GCS score at admission, cerebral herniation, NICU length of stay and transfusion of red blood cells were risk factors for IDDVT and its extension. The D-dimer level during the NICU stay was not a sensitive predictor of IDDVT occurrence. The elevation of D-dimer by a factor of 4 on days 5-7 compared to day 1 of the NICU stay was a predicator of IDDVT extension (the area under the ROC was 0.81 , and the sensitivity and specificity were 0.77 and 0.75 , respectively).

\section{Conclusions}

The occurrence and extension of IDDVT are common in ABI patients during the perioperative period, particularly in elderly male patients with severe cases. Changes in D-dimer may be helpful for diagnosing IDDVT extension in ABI patients. 


\title{
Poster 251
}

\section{Arginase-1 Microglia and Efferocytosis after Murine Neonatal Brain Hypoxia-ischemia}

\author{
Jana K. Mike, Donna M. Ferriero
}

Department of Pediatrics, University of California San Francisco, San Francisco, CA, United States

\section{Background \& Purpose}

Arginase-1 (Arg1) is the key regulatory enzyme of inflammation and tissue repair. Arg1 is widely used as a marker of "pro-repair" microglia and macrophages (Mi/Ma) and recent studies suggest a possible role for ARG-1 in regulation of Mi/Ma efferocytosis, a process of phagocytic clearance of apoptotic cells that prevents activation of inflammation. Little is known about efferocytosis after neonatal brain hypoxiaischemia (HI).

\section{Methods}

We exposed C57BL/6 (wild-type) mice to hypoxia-ischemia on postnatal day 9, as follows: permanent coagulation of left common carotid artery to induce ischemia, a 1-h recovery period and exposure to 10 $\%$ oxygen/balance nitrogen at $37^{\circ} \mathrm{C}$ for $50 \mathrm{~min}$ to induce hypoxia. Animals were perfused at $1 \mathrm{~h}, 4 \mathrm{~h}, 12 \mathrm{~h}$, day $1,3,5,8,14$ and 50 with $4 \%$ paraformaldehyde, brains were post-fixed, sectioned on a cryostat (12 um) and examined histologically with Cresyl-violet staining to assess the degree of damage and ARG1+Mi/Ma spatiotemporal localization via immunohistochemistry (ARG-1+Mi/Ma =ARG-1+lba-1+cells).

\section{Results}

ARG-1+Mi/Ma localized to the ventral brain (cortical subplate, olfactory areas). The number of ARG$1+\mathrm{Mi} / \mathrm{Ma}$ was highest early in development (P9) and decreased with age (P14,P23) to undetectable in adult mice (P50). $\mathrm{HI}$ reactivated at least some expression of ARG-1 in adult mice as we have detected a few ARG-1+Mi/Ma at the injury site. Cresyl-violet staining in our model shows injury located predominantly in the cortex and hippocampus, the HI caused accumulation and persistence of ARG$1+\mathrm{Mi} / \mathrm{Ma}$ in the $\mathrm{HI}$ injured cortex and striatum. ARG-1+Mi at the injury site touched, enwrapped and engulfed dead neurons and expressed PPAR $\gamma$ suggesting the role of ARG-1+Mi/Ma in phagocytosis of dead/dying neurons.

\section{Conclusions}

ARG-1+Mi/Ma form a unique population located in specific anatomical areas of the neonatal brain. While the precise role of ARG-1+Mi/Ma remains unknown, ARG-1+Mi/Ma may play a role in regulation of efferocytosis specifically for the neonatal brain. 
Poster 252

\section{Association of Invasive Brain Tissue Oxygenation and Non-Invasive Cerebral Regional Oximetry After Pediatric Severe Traumatic Brain Injury}

Brian Appavu, Ann Oh, M'Hamed Temkit

Phoenix Children's Hospital, Department of Neurology, Phoenix, AZ, United States

\section{Background \& Purpose}

Cerebral oxygenation monitoring is important towards detection of brain tissue hypoxia that can impact outcomes after pediatric severe traumatic brain injury (TBI). Measurements of non-invasive cerebral regional oxygen saturation ( $\mathrm{rSO} 2$ ) and invasive brain tissue oxygenation ( $\mathrm{PbtO} 2$ ) are methods of investigating markers of cerebral oxygenation. Cerebral oxygenation can be impacted by changes in physiologic covariates such as intracranial pressure (ICP), arterial blood pressure (ABP) and end-tidal carbon dioxide content (EtCO2). In this study, we aimed to identify the association between $\mathrm{rSO} 2$ and $\mathrm{PbtO} 2$ as well as the influence that ICP, ABP and EtCO2 have on their values.

\section{Methods}

This is a single-institution, retrospective cohort study of eleven children with severe TBI at a single institution that underwent multimodality neurologic monitoring in the pediatric intensive care unit. Continuous synchronized recordings of $\mathrm{rSO} 2$, PbtO2, ICP, ABP and EtCO2 data was captured in 5-hour epochs. Grouped analysis was performed on all patients using multivariate dynamic structural equations modeling (DSEM) to investigate the association of $\mathrm{rSO} 2$ and $\mathrm{PbtO} 2$ with each other in addition to understanding the influence that ICP, ABP and EtCO2 have on their values. The strength of associations was summarized using standardized coefficients and corresponding $95 \%$ credible intervals. Statistical significance was based on the interval not containing 0 .

\section{Results}

$\mathrm{PbtO} 2$ and $\mathrm{rSO} 2$ values were positively associated with each other (estimate $=0.044,95 \%$ credible interval $[0.039,0.050])$. ICP was more positively associated with $\mathrm{rSO} 2(0.333[0.327,0.339])$ than PbtO2 $(0.012[0.008,0.017])$. ABP was positively associated with PbtO2 $\{0.359[0.355,0.363]\}$ and negatively associated with $\mathrm{rSO} 2(-0.120[-0.125,-0.114])$. EtCO2 was positively associated with $\mathrm{rSO} 2(0.198[0.188$, $0.208])$ and not associated with PbtO2 (0.008 [-0.001, 0.017]).

\section{Conclusions}

After pediatric severe $\mathrm{TBI}, \mathrm{PbtO} 2$ and $\mathrm{rSO} 2$ values are associated with each other but vary considerably in their association with physiologic parameters that influence brain tissue oxygenation. 


\title{
Poster 253
}

\section{Deviations from NIRS-Derived Optimal Blood Pressure are Associated with Worse Outcomes after Pediatric Cardiac Arrest}

\author{
Matthew P. Kirschen, Tanmay Majmudar, Forrest Beaulieu, Ryan Burnett, Mohammed Shaik, Ramani \\ Balu, Todd Kilbaugh, Ramon Diaz-Arrastia, Robert Berg, Alexis Topjian \\ Department of Anesthesiology and Critical Care Medicine, The Children's Hospital of Philadelphia, \\ Philadelphia, PA, United States
}

\section{Background \& Purpose}

Secondary brain injury occurs after cardiac arrest due to impaired cerebrovascular autoregulation (CAR) leading to compromised cerebral perfusion. Objectives were to evaluate CAR using NIRS and arterial blood pressure during the first 24 hours after arrest and to determine if deviations from optimal mean arterial pressure (MAPopt) were associated with outcomes.

\section{Methods}

Retrospective study of children who had cardiac arrest and synchronized invasive arterial blood pressure and cerebral NIRS monitoring. CAR was quantified by a moving, linear correlation between MAP and rSO2 (COx). COx $>=0.3$ indicated impaired CAR. MAPopt is the COx-derived MAP where CAR is most intact, and was calculated using a multi-window weighted algorithm and updated every minute. We calculated the burden (magnitude and duration) of MAP less than $5 \mathrm{mmHg}$ below MAPopt, as the area between MAP and MAPopt -5 curves, normalized over the monitoring duration. Unfavorable outcome was defined as $>=1$ change in pediatric cerebral performance category (PCPC) from baseline resulting in hospital discharge PCPC score 3, 4, 5, or 6 . We used univariate regression to test the association between burden of MAP less than MAPopt-5 and outcome.

\section{Results}

Twenty-nine children with a median age of 2.5 [IQR 1.5,13.8] years were evaluated. Patients spent $35 \%$ $[20,44]$ of the first 24 hours post-arrest with COx $>=0.3$. MAPopt was equivalent to the 70 th $[55,90]$ percentile of MAP for age. Patients spent 35\% [24,60] of time below MAPopt-5. Eighteen patients had an unfavorable outcome. Patients with an unfavorable outcome had a higher burden of MAP less than MAPopt-5 than patients with a favorable outcome $(219[108,358]$ vs $81[52,107] \mathrm{mmHg} * \mathrm{Min} / \mathrm{Hr}$; OR 4.0 [95\% $\mathrm{Cl} 1.14$ to 32.23$])$.

\section{Conclusions}

The optimal MAP was a median of the 70th percentile for age. Greater burden of MAP below NIRSderived optimal MAP was associated with unfavorable outcomes in children after cardiac arrest. 
Poster 254

\section{Diffusion-Weighted MRI is Associated With Outcome after Pediatric Cardiac Arrest}

Matthew P. Kirschen, Jeffrey I. Berman, Hongyan Liu, Heather Griffis, Antara Mondal, Cindee Levow, Madeline Winters, Ramani Balu, Shih-Shan Lang, Jimmy Huh, Todd Kilbaugh, Ramon Diaz-Arrastia, Robert A. Berg, Arastoo Vossough, Alexis Topjian

Department of Anesthesiology and Critical Care Medicine, The Children's Hospital of Philadelphia, Philadelphia, PA, United States

\section{Background \& Purpose}

Diffusion MRI in the first 7 days after cardiac arrest (CA) is sensitive to identify hypoxic-ischemic brain injury. Our objective was to determine association between whole brain mean apparent diffusion coefficient (ADC) and outcomes after pediatric CA.

\section{Methods}

Retrospective study of children who had CA between 2008 and 2019 and a clinical MRI performed within 7 days of arrest. After computing an ADC map for each patient, we determined whole brain mean ADC and the percentage brain volume with voxels below an ADC of $650 \times 10-6 \mathrm{~mm} 2 / \mathrm{s}$. ADC z-scores by age were calculated via fitting an exponential model to a local dataset of whole brain mean ADC in normal children. Unfavorable outcome was defined as $>=1$ change in pediatric cerebral performance category (PCPC) from baseline resulting in hospital discharge PCPC score 3, 4, 5, or 6 . Logistic regression was used to test the association between age-adjusted whole brain mean ADC and outcome, accounting for time between CA and MRI.

\section{Results}

We measured ADC from 137 patients (median age 2.6 [IQR 0.6,8.7] years) performed a median of 3 [2,5] days post-CA. Eighty-seven (64\%) patients had an unfavorable outcome. After accounting for time between $\mathrm{CA}$ and $\mathrm{MRI}$, lower whole brain mean ADC z-score was associated with higher odds of unfavorable outcome (OR 2.17, 95\%Cl 1.47,3.57, p<0.001). Unfavorable outcome was associated with a greater percentage of brain tissue below an ADC of $650 \times 10-6 \mathrm{~mm} 2 / \mathrm{s}$, (unfavorable vs favorable: $9.9 \%$ $[2.4,32.3]$ vs $1.1 \%[0.1,2.8], p<0.001)$. A prespecified threshold of $>10 \%$ of brain tissue with ADC less than $650 \times 10-6 \mathrm{~mm} 2 / \mathrm{s}$ had a sensitivity of $49 \%$ and specificity of $92 \%$ (AUROC 0.71 ) for unfavorable outcome.

\section{Conclusions}

In a large single-center cohort, lower age-adjusted whole brain mean ADC, indicating more extensive hypoxic-ischemic brain injury, was associated with unfavorable outcomes after pediatric cardiac arrest. 


\title{
Poster 255
}

\section{Evaluation of Epidiolex ${ }^{\circledast}$ (Cannabidiol) on Seizure-Related Emergency Department and Hospital Admissions}

\author{
Renad Abu-Sawwa, Aaron Chase, Yong Park \\ University of Florida College of Pharmacy, Gainesville, FL, United States
}

\section{Background \& Purpose}

Intractable seizure disorders are common and lack many effective treatment options. Many patients have poor outcomes and frequently utilize healthcare resources. Cannabidiol (CBD) was recently approved for use in for a few intractable seizure syndromes and has demonstrated potential as a highly effective treatment option. To date, there is no data on the impact of CBD on healthcare resource utilization. The objective of this study was to determine how CBD affects seizure-related emergency department (ED) and hospital admissions.

\section{Methods}

This single center retrospective cohort study included patients $\geq 1$ year on CBD, excluding participants in CBD clinical trials or on $<6$ months of therapy. Demographics, anti-seizure medications (ASM), ED and hospital admissions were collected from the electronic medical record. Co-primary outcomes included change in seizure-related ED and hospital admissions. Secondary outcomes included change in ASMs and total ED or hospital admissions. Co-primary outcomes were assessed using generalized linear modeling. Descriptive statistics were used to analyze all other variables.

\section{Results}

In the one-hundred total patients, there was no difference in either seizure-related ED visits (0.012 vs $0.011, p=0.85$ ) or hospital admissions per month ( 0.019 vs $0.021, p=0.7$ ). However, given the low percentage of the total cohort $(n=100)$ with either a seizure-related ED visits and hospital admissions ( $9 \%$ and $18 \%$, respectively), a subgroup analysis was conducted. Those with seizure-related hospital admissions prior to CBD $(n=18)$ had significantly less seizure-related hospital admissions after initiation of CBD (23 admissions [0.104 per month] vs 15 admissions [0.055 per month], $p=0.007$ ).

\section{Conclusions}

Despite the lack of statistically significant difference in primary outcomes, CBD may have a potential for a clinically beneficial impact on those patients with prior seizure-related admissions, who are the highest utilizers of healthcare resources. 
Poster 256

Implementation of an Institutional Protocol for Rapid Recognition of Stroke in VAD Patients

Michele Mills, Allison Reichhold, Kim Kato, Anna Joong, Jonathan Kurz

Ann and Robert H. Lurie Children's Hospital of Chicago; Department of Neurology, Chicago, IL, United States

\section{Background \& Purpose}

Ventricular assist devices (VAD) are now commonly used as a bridge to heart transplant in the pediatric population. Although this is a life-saving therapy, VAD-associated neurologic dysfunction is common, occurring in more than $20 \%$ of patients. Mechanical thrombectomy is an emerging treatment for pediatric stroke. With high risk for embolic stroke in pediatric VAD patients, and case reports of successful mechanical thrombectomy in this population, we sought to improve our institutions recognition and diagnosis of acute stroke.

\section{Methods}

Representatives from neuro-critical care, heart failure, the cardiac intensive care, and nursing partnered to increase stroke awareness. Education was targeted to providers, nurses, and therapists via case conferences and nursing education. Bi-yearly VAD-specific education highlighted neurologic concerns. Bedside documents were made with daily VAD goals, a nursing neurologic exam and frequency of neuromonitoring. Nursing neurologic exams were established based on patient's intubation status and ability to follow commands, with a goal of identifying mental status changes and new focal weakness. Exam frequency varied as patients transitioned from the acute to stable phases of care. Additionally, the neuro-critical care team scheduled protocolized assessments based on neurologic risk. To speed time to imaging a VAD-specific stroke algorithm and order set were established.

\section{Results}

Since 2015, 46 patients had VADs placed at our institution, 25 prior to the intervention and 21 postintervention. Pre-intervention, 8 patients were evaluated for stroke. Mean time to imaging was 7 hours with three cases having no last known normal. Post-intervention, 6 patients were evaluated for stroke. Time to imaging has decreased to 2.8 hours and all patients had a documented last known normal. On review it was also noted that use of Head CTs decreased from 11, pre-intervention to 3 postintervention

\section{Conclusions}

Development of a VAD protocol decreased time to imaging for suspected stroke and reduced unnecessary imaging studies. 


\section{Poster 257}

\section{Neuromonitoring Practice is Highly Variable in Pediatric Extracorporeal Membrane Oxygenation}

Christopher M. Ruzas, Giulia M. Benedetti, Craig A. Press, John Kim, Jonathan E. Kurz, Zach Daniels, Craig M. Smith, Mark S. Wainwright

University of Colorado School of Medicine, Department of Pediatrics, Division of Critical Care Med, Aurora, CO, United States

\section{Background \& Purpose}

To determine current neuromonitoring practices, neurocritical care and resource availability for pediatric centers providing extracorporeal membrane oxygenation (ECMO).

\section{Methods}

A survey was distributed to the Pediatric Neurocritical Care Research Group. One survey per institution was collected. Responses were categorized based on presence of institutional neuromonitoring guidelines (NGs), ECMO volume and protocolized neurologic consultation. Data are reported descriptively and comparisons by chi-square statistics.

\section{Results}

The response rate was 32\% (25/78) including 14 high-volume centers (>30 runs/year). Neuromonitoring guidelines (NGs) were reported by $60 \%$ of centers. A greater number of high-volume centers reported use of NGs (79\% vs 36\%; $p<0.05$ ). Modalities routinely utilized were head ultrasound (HUS) $84 \%$, cNIRS $(68 \%)$ and cEEG (44\%). Neither ECMO volume nor the presence of NGs impacted the reported routine use of specific neuromonitoring modalities. Protocolized neurologic consultation was associated with NGs $(60 \%, 9 / 15$ vs $0 \%, 0 / 10 ; p=0.002)$. Routine neurology consults were associated with universal use of NGs $(p<0.01)$ and protocolized cEEG $(p<0.001)$. HUS practice varied, with most reporting protocolized use daily/every other day (36\%) for the duration, or daily for first 3-5 days (44\%) of ECMO. In centers reporting protocolized CEEG, duration of recording was 1-2 days. Routine post-ECMO MRI was reported by $20 \%$ of centers.

\section{Conclusions}

Neuromonitoring modalities are widely available at surveyed ECMO centers. High volume centers were more likely to report NGs. NGs correlate with increased involvement of neurologic consultants, though not routine use of specific modalities. All centers with routine neurologic consultation in ECMO patients had standardized NGs and all performed protocolized cEEG. Post-ECMO MRI imaging is not common. Silent neurologic injury in patients receiving ECMO is likely underrecognized and potentially progresses to clinically significant neurologic injury. Efforts are needed to determine and promote standardization of evidenced-based neuromonitoring practices to detect, prevent and treat acute neurologic injury. 


\section{Poster 258}

\section{Pediatric ECMO Survivors Experience Mild Neurobehavioral Impairment Years After Decannulation}

Ashley Turner, Ashley Turner, Madison Streb, Amy Ouyang, Skyler Leonard, Trevor Hall, Cydni Williams, Ahmed Said, Kristin Guilliams

Washington University in St. Louis - School of Medicine, Department of Pediatrics, St. Louis, MO, United States

\section{Background \& Purpose}

There are limited reports of neurobehavioral outcomes of children supported on extracorporeal membrane oxygenation (ECMO) in the United States more than 12 months following decannulation. We hypothesized young ECMO survivors may have long-term neurobehavioral impairment.

\section{Methods}

Neonatal and pediatric ECMO survivors $>12$ months post-decannulation were recruited at two tertiary centers to complete a neurobehavioral assessment of adaptive skills using the Vineland Adaptive Behavior Scales, Third Edition (VABS-III) by caregiver or patient interview. U.S. population norms for the VABS-III are $100+/-15$ with higher scores indicating higher function. VABS-III composite scores were compared between subpopulations with the Mann-Whitney $U$ test. Additional patient and ECMO characteristics were collected from the medical record.

\section{Results}

Forty-two ECMO survivors (64\% male) who were cannulated at 0.0-19.8 (median: 1.7) years completed the VABS-III 1.3-12.8 (median: 5.5) years following ECMO decannulation. The mean VABS-III composite score for the entire ECMO cohort was 82.0 (range: 31-115) which is greater than 1 SD below the normative population. There was no significant difference in VABS-III scores for patients requiring venovenous ( $n=6)(94.5$ [IQR 69.0, 105.5]) compared to veno-arterial ECMO $(n=32)(81.5$ [IQR 69.3, 97.8]) $(p=.33)$. However, patients who required ECPR $(n=12)(66.0$ [IQR $54.3,78.0])$ had significantly lower VABS-III scores than those without ECPR $(89.5$ [IQR 74.8, 101.5]) $(p=<.01)$. Patients with congenital heart disease $(n=15)$ (73.0 [IQR 54.0, 89.0]) had significantly lower VABS-III scores than those without congenital heart disease (88.0 [IQR 78.0, 101.0]) $(p=<.01)$. Among patients with electroencephalography data $(n=23)$, ECMO survivors with electrographic seizures while on ECMO $(n=4)(54.5$ [IQR: 48.0, 66.3]) had significantly worse VABS-III scores than those without seizures (91.0 [IQR: 73.0, 100.0]) ( $p<.01)$.

\section{Conclusions}

This extended follow-up study demonstrates pediatric ECMO survivors suffer mild neurobehavioral impairment years after decannulation. Factors including ECPR, pre-existing congenital heart disease, and seizures while on ECMO are associated with worse neurobehavioral outcomes. 


\title{
Poster 259
}

\section{Post-traumatic Seizures in Children with Abusive Head Trauma}

\author{
Mahjabeen Khan, Sean Goretzke, Nandini Calamur
}

Department of Pediatrics and Neurology, Saint Louis University School of Medicine, Saint Louis, MO, United States

\section{Background \& Purpose}

Abusive head trauma (AHT) causes death and disability in children. Post-traumatic seizures (PTS) are common in AHT and cause secondary neurological injury. The study of the characteristics and true burden of PTS is important to develop effective management strategies. Our objectives were to study the incidence and risk factors for PTS, utilization of continuous EEG (cEEG), management with antiepileptic drugs (AEDs) and clinical outcomes in victims of AHT.

\section{Methods}

We assembled an eleven-year cohort of children aged 0-3 years, who suffered AHT and were subsequently admitted to an academic children's hospital. Data pertinent to demographics, clinical features, management and follow up were collected by retrospective chart review and analyzed.

\section{Results}

Sixty out of ninety-two AHT victims developed PTS during hospitalization. Mean age was $6 \pm 5.8$ months and predominantly consisted of males (65\%). Factors associated with higher likelihood of seizures were subdural hematoma (OR 5.32 [95\% Cl 1.27-22.2]) and comorbidities like prematurity (OR 5.45 [95\% Cl 1.16-25.48]). Over $90 \%$ of PTS, occurred within a week of hospitalization. Of those, only three cases had purely electrographic seizures. Mean time to cEEG initiation was 14.7 hours. Nearly $50 \%$ of seizures continued sub-clinically, despite clinical control. Although levetiracetam was most commonly used, monotherapy with the same was ineffective in $>50 \%$. In comparison to AHT patients without seizures, PTS patients had longer ICU and hospital stays, by 4.1 and 7.8 days respectively. Although, majority (80\%) were discharged on AEDs, $40 \%$ were weaned off at follow-up. Among PTS patients, mortality rate was $<5 \%$ with the most common long-term sequelae being developmental delay and epilepsy.

\section{Conclusions}

To date, this is the largest cohort study to examine PTS in young AHT victims. While most PTS were clinical and occurred early, cEEG monitoring was essential in detection and treatment of subclinical seizures. Overall, PTS had a good survival rate and outcomes. 
Poster 260

The Association of Late Neurosurgical Interventions on Cerebral Physiology After Pediatric Traumatic Brain Injury

Brian Appavu, Phillip Tarzi, M'Hamed Temkit, P. David Adelson

Phoenix Children's Hospital, Department of Neurology, Phoenix, AZ, United States

\section{Background \& Purpose}

Late neurosurgical interventions (NSI) such as decompressive craniectomy or external ventricular drainage (EVD) may be needed in the treatment of refractory intracranial hypertension (ICH) after pediatric traumatic brain injury (TBI). While such interventions may aid in reduction of ICH, the effect of late intervention on cerebrovascular pressure reactivity (CVPR) and systemic physiology remains unclear.

\section{Methods}

Pediatric patients with TBI who had received NSI more than 24 hours after initial injury and had multimodal neurologic monitoring with intraparenchymal intracranial pressure (ICP) monitoring were included. Univariate dynamic structural equations (DSEM) were used to determine differences in estimated means of arterial blood pressure (ABP), heart rate (HR) and ICP within 4-hour epochs before and after NSI. Univariate DSEM was also applied to investigate associations of ABP, HR and ICP within the same epochs before and after NSI.

\section{Results}

Six children with STBI were identified who had late NSI, including four children who received decompressive craniectomies and two children had placement of EVD. All six patients (100.0\%) had reduction in the standardized means of ICP after NSI (median $8.5 \mathrm{mmHg} \pm$ [interquartile range] 11.9), whereas four patients (66.7\%) had reduction in ABP (median $6.1 \mathrm{mmHg} \pm 7.2$ ) and two patients (33.3\%) had reduction of HR after intervention (median 92.3 beats per minute \pm 7.2 ). Five patients (83.3\%) had positive association of ABP and ICP prior to NSI, all of whom had either a reduction in strength of positive association or reversal of positive association to negative association after NSI. One patient demonstrated a negative association of $A B P$ and ICP prior to NSI which reversed to a positive association after decompressive craniectomy.

\section{Conclusions}

In children with refractory ICH and impaired CVPR, late NSI can reduce ICP and improve CVPR. Further work is needed to understand whether such interventions worsen CVPR in those patients without intact CVPR. 


\section{Poster 261}

\section{Association of Epileptiform Activity and Burden with Outcomes in Toxic-Metabolic Encephalopathy}

Patrick M. Chen, Jin Jing, Wending Ge, Eric S. Rosenthal, M. Brandon Westover, Sahar F. Zafar

Massachusetts General Hospital, Department of Neurology, Boston, MA, United States

\section{Background \& Purpose}

Toxic metabolic encephalopathy is a diffuse cerebral dysfunction common in critically ill patients. Periodic and rhythmic epileptiform activity (EA) is commonly seen on continuous EEG (cEEG) in this cohort, yet its clinical significance is unknown. We characterize TME EA burden and its association with morbidity and mortality.

\section{Methods}

Retrospective analysis of TME patients undergoing >18 hours of EEG between 2012 and 2017. Patients with acute brain injury and cardiac arrest were excluded. EA were defined as seizure activity, and periodic and rhythmic patterns excluding GRDA. We classified the highest EA burden in a 12-hour epoch as high ( $>50 \%$ ) or low burden $(<50 \%)$. Outcomes measured were mortality and modified Rankin scale (mRS) at discharge.

\section{Results}

One-hundred and sixteen patients met study criteria with $34 \%$ having a primary renal diagnosis. Discharge mortality was $36 \%$ (41); $86 \%$ (99) had poor outcomes (mRS 4-6). $21 \%$ of patients had electrographic seizures and 35\% (41/116) had high EA burden. High EA burden was associated with higher APACHE II score (median= 21, IQ [17-31] vs. median =19, IQ [14-23]) but not length of stay or mechanical ventilation time when compared to patients with low EA burden. In multivariable analysis adjusted for covariates (age, APACHE II, Charlson Comorbidity Index, primary diagnosis), increasing EA burden was associated with poor outcome (Coef: 2.6 [0.14-5.1], $p=0.038$ ). Increase in peak EA burden from $0-100 \%$ increased probability of poor outcome discharge outcome by $25 \%$.

\section{Conclusions}

Increasing burden of EA is associated with worse discharge outcomes in patients with TME. Future studies are needed to determine whether treatment with anti-seizure medications vs. correction of underlying metabolic derangement are more likely to improve outcomes. 
Poster 262

\section{Cerebrospinal Fluid Findings in Patients with Seizure in the Setting of COVID-19: A Review of the Literature}

Elizabeth Carroll, Kara R. Melmed, Jennifer Frontera, Dimitris G. Placantonakis, Steven Galetta, Laura Balcer, Ariane Lewis

NYU Langone Health, New York, NY, United States

\section{Background \& Purpose}

We reviewed the literature on cerebrospinal fluid (CSF) studies in patients who had a seizure in the setting of COVID-19 infection to evaluate for evidence of viral neuroinvasion.

\section{Methods}

We performed a systematic review of Medline and Embase to identify publications that reported one or more patients with COVID-19 who had a seizure and had CSF testing preformed. The search ranged from December 1st 2019 to November 18th 2020.

\section{Results}

We identified 56 publications which described 69 unique patients who met our inclusion criteria. Of the 54 patients whose past medical history was provided, $2(4 \%)$ had epilepsy and $1(2 \%)$ had a prior seizure in the setting of hyperglycemia, but the remaining 51 (94\%) had no history of seizures. Seizure was the initial symptom of COVID-19 for 15 (22\%) patients. There were $26(40 \%)$ patients who developed status epilepticus. SARS-CoV-2 PCR testing was performed in the CSF for 45 patients; 6 (13\%) had a positive CSF SARS-CoV-2 PCR, only 1 (17\%) of whom had status epilepticus. The cycle thresholds were not reported. Evaluation for CSF SARS-CoV-2 antibodies (directly or indirectly, via testing for CSF oligoclonal bands or immunoglobulins) was performed in 26 patients, only 2 (8\%) of whom had evidence of intrathecal antibody synthesis. Of the 11 patients who had CSF autoimmune antibody panels tested, 1 had NMDA antibodies and 1 had Caspr-2 antibodies.

\section{Conclusions}

Detection of SARS-CoV-2 in the CSF of patients with seizures who have COVID-19 is uncommon. Our review suggests that seizures in this patient population are not likely due to direct viral invasion of the brain. 


\title{
Poster 263
}

\section{Continuous EEG Monitoring in Evaluation of Paroxysmal Events: Yield and Clinical Impact in ICU}

\author{
Eugenie Atallah, Chen Hai
}

George Washington University, Department of Neurology, Washington DC, United States

\section{Background \& Purpose}

Continuous EEG (CEEG) has been widely used to evaluate altered mental status and diagnose nonconvulsive seizures in ICU. We aim to investigate the yield of CEEG and predictor factors in evaluating paroxysmal events. In addition, clinical impact of cEEG was evaluated.

\section{Methods}

We identified patients in the ICU who underwent cEEG monitoring ( $>6 \mathrm{~h}$ ) to evaluate paroxysmal events between Jan 1, 2018 and Jan 31, 2019. We extracted patient characteristics including medical history, neurological examination, and image findings. The primary reason and duration of each EEG recording was also obtained. The EEG findings were dichotomized into diagnostic and non-diagnostic. A diagnostic EEG included a recording with the finding of an epileptiform discharge, seizure or a habitual event (epileptic or non-epileptic event). To assess the impact of cEEG on treatment, we recorded changes in anti-seizure medication (ASM) after CEEG studies.

\section{Results}

Seventy-six recordings were identified and indications for cEEG included motor-related events ( $n=52)$, gaze deviation $(n=8)$ and others $(n=16)$, such as speech changes, behavior changes, hallucinations, and body stiffness. Diagnostic CEEG studies accounted for $26 \%(20 / 76)$ recordings which included EEG with epileptiform findings (16/76) and EEG with habitual non-epileptic events (4/76). The history of epilepsy is the only predictor for the diagnostic finding $(p=0.004)$. The average duration of recording was 48 hours and the initial abnormal finding occurred within 6 hours of the recording in most patients (13/20, $65 \%)$. Totally 22 patients had ASM changes after cEEG studies. ASM changes (decrease or increase) were noted in $65 \%(13 / 20)$ patients with the diagnostic EEG findings, whereas $16 \%(9 / 56)$ patients discontinued ASM after the non-diagnostic CEEG

\section{Conclusions}

cEEG is valuable in evaluating paroxysmal events, and a history of epilepsy is associated with the positive finding. Both diagnostic and non-diagnostic CEEG lead to ASMs adjustments. 
Poster 264

\section{Experience with Brivaracetam in the Acute Care Setting at a Large Tertiary Care Center}

Justine Cormier, Christopher Traner, Thanujaa Subramaniam, Gabriella Garcia, Lawrence Hirsch, Abdalla Ammar, Emily Gilmore

Yale New Haven Hospital Division of Neurocritical Care, New Haven, CT, United States

\section{Background \& Purpose}

To analyze usage patterns, efficacy, tolerability, and long-term continuation of brivaracetam (BRV) when initiated in the acute, inpatient setting at a large, academic institution.

\section{Methods}

A retrospective chart review was conducted on patients who received BRV in the emergency or inpatient setting at our institution from 2017 to 2021. Patients under 18 years of age or taking BRV prior to presentation were excluded.

\section{Results}

Data from 133 patients were included in the analysis. BRV was used for treatment of seizures or status epilepticus (SE) in $77 \%(\mathrm{~N}=103)$, rhythmic or periodic patterns (RPPs) in $21 \%(\mathrm{~N}=28)$, and seizure prophylaxis in $2 \%(\mathrm{~N}=3)$. BRV was the first anti-seizure medication (ASM) tried in a small number of patients (7\%), with the majority (93\%) having tried other ASM first (median ASM prior to BRV = 2 (IQR 15)). Of those who tried another ASM first, BRV was added due to intolerance of other ASMs in $16 \%$ $(\mathrm{N}=20)$; behavioral changes were most common $(35 \%, \mathrm{~N}=7)$. A small number of patients $(\mathrm{N}=14)$ received an initial dose of $25-75 \mathrm{mg}$, while the majority $(\mathrm{N}=119)$ received $100-400 \mathrm{mg}$ followed by a median maintenance dose of $200 \mathrm{mg} /$ day (IQR $50-600 \mathrm{mg} /$ day). Of patients with seizures or SE monitored with continuous EEG (113), 64\% saw clinical and/or electrographic improvement with BRV, while $26 \%$ returned to baseline with resolution of seizures or SE. BRV was discontinued in $37 \%$ of patients $(\mathrm{N}=50)$, most commonly due to inefficacy (36\%), with only one report of behavioral side effects. Of 78 patients discharged on ASMs, 59 (77\%) were discharged on BRV. Follow-up data were available for 53 patients, of which 51 remained on one or more ASM, including 33 (65\%) who remained on BRV.

\section{Conclusions}

BRV appears to be a safe, well-tolerated, and effective therapy for acute, inpatient management of seizures, SE, and RPPs. Future studies validating these findings are warranted. 


\section{Poster 265}

\section{Immunomodulatory Therapy in the Treatment of Refractory Status Epilepticus: A Case Series}

Matthew R. Woodward, Huanwen Chen, Melissa Motta, Jamie Podell, Wan-tsu Chang, Gunjan Parikh, Neeraj Badjatia, Nicholas A. Morris

Department of Neurology, University of Maryland School of Medicine, Baltimore, MD, United States

\section{Background \& Purpose}

Immunomodulatory therapy (IT) has been reported as a possible treatment for refractory status epilepticus (RSE) in case reports or small series. We describe a cohort of patients treated with IT for RSE and the effect of IT on seizure cessation, clinical exam and adverse events (AEs).

\section{Methods}

We performed a chart review of patients admitted to the neurocritical care unit with RSE from 20162020, identifying patients that received IT (methylprednisolone $1000 \mathrm{mg}$ per day, intravenous immunoglobulin [IVIG], plasmapheresis) for the primary indication of RSE or recurrent seizures despite treatment. Patients were excluded if IT was used for an alternative indication. The primary outcome was seizure cessation or electrophysiological improvement of highly ictal electroncephalographic patterns not meeting criteria for seizure. Secondary outcomes were frequency of IT-related AEs and change in Glasgow coma scale (GCS) pre- and post-IT. Wilcoxon rank-sum test was used to compare GCS.

\section{Results}

We identified 19 patients (median age: 57 [IQR: 26-68], 12 female) treated with IT (methylprednisolone [ $n=13]$, IVIG [ $n=2]$, plasmapheresis [ $n=12$ ], combination therapy [n=9]). Etiology of RSE included cryptogenic $(n=13)$ and one case each of antibody confirmed auto-immune encephalitis, genetic, infectious, structural, and primary generalized epilepsy. At the time of IT (median 7 days postseizure/RSE onset [IQR: 5-11]), 12 had ongoing seizures/RSE; two were in burst suppression and four had highly ictal electroncephalographic patterns. Of the 12 patients with ongoing seizures/RSE, seizure cessation occurred during IT in eight cases, and following IT in four. The median GCS post-IT was six (IQR: 3-11), versus three pre-IT (IQR: 3-5) ( $Z=68, p=0.02)$. Adverse events related to IT occurred in 10 patients, including hypotension requiring vasopressors, line-associated thrombosis and line-associated infection.

\section{Conclusions}

Our results suggest IT may be an effective adjunctive therapy in treating RSE, though potentially limited by frequent AEs. Future research is needed to determine the proper indications for IT in RSE. 
Poster 266

\section{Investigation of Clinical Seizures and EEG Patterns Following LVAD Placement}

Elizabeth Carroll, Manisha Holmes, Jennifer A Frontera, Nader Moazami, Alex Reyentovich, Kara Melmed

NYU Langone Health, New York, NY, United States

\section{Background \& Purpose}

Neurologic events are a devastating complication in patients with left ventricular assist devices (LVADs). There is minimal literature exploring seizure and electroencephalography (EEG) in this patient population.

\section{Methods}

We performed a retrospective cohort study of patients admitted to our hospital from 2011 to 2020 following LVAD placement, identified via the INTERMACS database. We reviewed all patients with a documented neurologic event for the documentation of clinical seizure or the use of EEG during admission. We obtained factors relevant to the clinical seizure via chart review, including medical history, concomitant neurologic events such as ischemic or hemorrhagic stroke, and antiseizure treatment. EEGs were reviewed to describe the distribution of various EEG findings.

\section{Results}

Of the 117 patients identified, 39 patients suffered a neurologic event, of which 19 were reported to have had a clinical event concerning for seizure, and/or underwent EEG. EEG or seizure occurred an average of 1.9 years (range 1 day -7.3 years) following LVAD placement. Only one patient had a history of epilepsy. Indication for EEG varied, with 7 (50\%) patients requiring EEG for characterization of paroxysmal events, 4 (29\%) for encephalopathy and concern for subclinical seizures, 2 (14\%) for postcardiac arrest hypothermia protocol, and $1(7 \%)$ for a seizure exacerbation. The most common electrographic pattern noted on EEG was slowing, which was seen in $6(43 \%)$ patients. Spontaneous epileptiform discharges were seen in 3 patients (21\%). Only $1(7 \%)$ patient had an electrographic seizure captured on EEG, and this patient was documented to be in convulsive status epilepticus. There were no patients that were diagnosed non-convulsive status epilepticus.

\section{Conclusions}

There has been minimal investigation into seizure and EEG patterns following LVAD placement. Larger scale studies evaluating which patients are at highest risk for seizure may be a direction of future study. 
Poster 267

\section{Pharmacodynamic Effects of Ketamine on Epileptiform Activity and Continuous Electroencephalography (cEEG) Frequency Spectra}

Megan E. Barra, Lisa D. Coles, Can Ozan Tan, Jaideep Kapur, James M. Chamberlain, Robert Silbergleit, Thomas P. Bleck, Andrew J. Cole, Shlomo Shinnar, James Cloyd III, Eric S. Rosenthal,

Department of Pharmacy, Massachusetts General Hospital, Boston, MA, United States

\section{Background \& Purpose}

We examined the effect of ketamine, an emerging therapy for status epilepticus (SE), on epileptiform activity and EEG frequency spectra to determine the onset and duration of its expected response (pharmacodynamic signature).

\section{Methods}

We evaluated frequency spectral power and epileptiform abnormality burden as pharmacodynamic responses in consecutive patients administered ketamine during cEEG monitoring for SE (04/201602/2021). Frequency-specific power (standardized to a 15-minute pre-administration baseline) and global spike count burden were measured (Persyst, Inc.) at baseline and post-administration 5-minute assessment periods, stratified by dose. A two-compartment pharmacokinetic model utilizing literature values linked to an Emax pharmacodynamic model examined associations between observed EEG changes and predicted serum concentrations.

\section{Results}

18 patients received bolus ketamine $(1.3 \mathrm{mg} / \mathrm{kg}$ [IQR 0.9, 1.6] median dose; 5 [4,7] median baseline number of antiseizure or anesthetic medications). The baseline spike count (median 25 [6, 216] spikes/300 seconds) changed by $-10[-81,3]$ at 30 minutes and $-15[-69,-5]$ at 60 minutes. Ketamine $\geq 1.5 \mathrm{mg} / \mathrm{kg}$ was associated with decreased power in the $1-4-\mathrm{Hz}, 4-8-\mathrm{Hz}, 8-13-\mathrm{Hz}$, and $13-20-\mathrm{Hz}$ bands in $89 \%, 78 \%, 67 \%$, and $56 \%$ of patients at 30 minutes and $89 \%, 78 \%, 56 \%$, and $56 \%$ at 60 minutes, respectively. The median differences from the baseline spike count for ketamine $\geq 1.5 \mathrm{mg} / \mathrm{kg}$ (median 89 $[18,273]$ spikes were $-20[-106,-20]$ at 30 minutes and $-32[-106,-32]$ at 60 minutes. Effects of ketamine $<1.5 \mathrm{mg} / \mathrm{kg}$ were less for both spike count and spectral power. EEG spectral changes commonly peaked at 30 minutes with duration of effect of 60-120 minutes. Pharmacodynamic modeling demonstrated a strong temporal association of estimated serum concentrations with EEG spectral changes.

\section{Conclusions}

Bolus administration of Ketamine at $\geq 1.5 \mathrm{mg} / \mathrm{kg}$ for SE resulted in maximum pharmacodynamic spike reduction at 30 minutes with changes in frequency spectra lasting 60 minutes, coupled to estimated serum concentrations. These pharmacodynamic findings may guide future clinical trials. 
Poster 268

Rapid Response EEG in Neuro-ICU: Single Center Experience

\section{Farid Sadaka}

Mercy Hospital St Louis/ Critical Care Medicine Department, St Louis, MO, United States

\section{Background \& Purpose}

The timely diagnosis and treatment of patients with seizures can prevent significant morbidity and mortality. Conventional EEG in the Neuro-ICU suffers from significant delays in access to EEG recordings and interpretation by skilled neurologists. Especially off-hours and on weekends and holidays, treatment decisions are made on the basis of clinical suspicion alone, which results in the potential for missing or undertreating non-convulsive seizures (NCS) and overtreating of patients without seizure activity leading to significant unnecessary intubations and increasing ICU and hospital length of stays. In this study, we report our experience with a recently FDA-cleared machine learning method (Clarity, Ceribell Inc.) that measures the burden of seizure activity in real time and generates bedside alerts for possible status epilepticus (SE).

\section{Methods}

A total of 76 adult patients underwent evaluation of possible seizures with rapid response EEG system (Rapid-EEG, Ceribell Inc.) over an 8-month period (09/2020-04/2021). Automated detection of seizure burden throughout a recording (calculated as the percentage of ten-second epochs with seizure activity in any 5-min EEG segment) was performed with Clarity ( $\geq 90 \%$ indicating $\geq 4.5$ min of seizure activity and triggering a status epilepticus alert). ALL EEGs were reviewed by a skilled epileptologist.

\section{Results}

A total of 76 Ceribell EEGs were performed (73 [96\%] during after-hours). Average recording duration was 6 hours. There were 7 EEGs (9\%) that generated alerts at the bedside for suspected seizure or status epilepticus ensuring a skilled neurologist could review the EEGs urgently. 14 EEGs (18\%) identified abnormal epileptiform activity but did not generate an alarm at the bedside. 55 EEGs (72\%) ruled out any seizure activity and prevented unnecessary treatment, sedation, and possible intubations.

\section{Conclusions}

Clarity provides timely detection of status epilepticus and is useful in diagnosis and treatment of patients with non-convulsive seizures and in preventing undertreatment or overtreatment of seizures. 


\section{Poster 269}

\section{Reduced Continuous EEG Electrode Arrays in Critical Care Settings: A Narrative Review}

Xiuxian Pham, Joshua Laing, Patrick Kwan, Piero Perucca, Terence John O'Brien, Andrew Udy

School of Public Health and Preventive Medicine, Monash University, Melbourne, Victoria, Australia

\section{Background \& Purpose}

Non-convulsive status epilepticus is a neurological emergency that can be difficult to diagnose and treat without continuous electroencephalography (CEEG). However, there are several challenges in utilising CEEG in critical care settings. One means to improving access is streamlining electrode arrays by exploring the diagnostic accuracy of reduced arrays compared to the current standard 10-20 system. This review examines the current literature on reduced electrode array models in critical care settings.

\section{Methods}

A literature review was conducted searching three electronic databases (MEDLINE, Embase and CENTRAL) up to April 2020, as well as by citation chaining, for studies comparing reduced electrode arrays to the current 21 leads standard in adults in acute care settings.

\section{Results}

1641 studies were identified, of which 13 studies were eligible and included in qualitative synthesis. Of these, 77\% (10/13) were retrospective studies and 92.3\% (12/13) were single-centred studies. Examined models ranged from using 4 to 12 leads, with variable setup strategies including hairline, forehead, circumferential and double interelectrode distance. Study objectives primarily revolved around seizure detection sensitivity and specificity with a reduced montage. While the objectives were largely similar across studies, the selection criteria and methodology varied substantially. Reduced array models demonstrated high specificity rates ranging from $86-100 \%$, but varied significantly in sensitivity at $54-$ $100 \%$ depending on the model and methodology. Overall, the majority of studies (12/13) favoured ongoing investigation and refinement of reduced electrode arrays given barriers in obtaining the standard setup in a timely and cost-effective manner for cEEG.

\section{Conclusions}

Compiled studies were heterogeneous in methodology and remains inconclusive as to the diagnostic strengths of reduced electrode arrays. While there is theoretical value in pursuing efficiency to improve cEEG availability in critical care settings, further investigations are necessary to determine the optimal system. 
Poster 270

\section{Salvage Electroconvulsive Therapy for the Treatment of Super Refractory Status Epilepticus}

Matthew R. Woodward, Seshagiri Doddi, Christopher Marano, Jennifer Pritchard, Stephanie Chen, Melissa Motta, Jamie Podell, Wan-tsu Chang, Gunjan Parikh, Neeraj Badjatia, Nicholas A. Morris

Department of Neurology, University of Maryland School of Medicine, Baltimore, MD, United States

\section{Background \& Purpose}

Electroconvulsive therapy (ECT) is a potential treatment for super-refractory status epilepticus (SRSE). We describe the largest known cohort of SRSE treated with ECT and describe its effect of ECT on seizure cessation, anesthetic burden, and adverse events.

\section{Methods}

We performed a retrospective chart review of patients admitted to the neurocritical care unit in SRSE and treated with ECT from 2014-2021. Electroconvulsive therapy occurred following anesthetic wean, consisting of 2-3 stimulations on each ECT day. The primary outcome was seizure cessation or electrophysiological improvement for patients with highly ictal encephalographic patterns not meeting criteria for seizure. Secondary outcomes were the change in dose of anesthetic agents in the $72 \mathrm{~h}$ preand post-ECT and frequency of ECT-related adverse events.

\section{Results}

We identified ten admissions among nine patients (median age: 57.5 [IQR: 43.3-69], seven female) that received ECT. At the time of ECT (median hospital day of first treatment 15 [IQR: 43.3-69]) six had ongoing SRSE; four were in burst suppression or had highly ictal electroencephalographic patterns and a poor neurological exam. Of the six patients with ongoing SRSE, seizure cessation occurred during ECT in three cases, $24 \mathrm{~h}$ post-ECT in one case, and $96 \mathrm{~h}$ post-ECT in one case. Four patients had eventual recurrence (median 5.5 days after last ECT). Reactivity, variability, or state change returned in three cases; frequency of highly ictal periodic patterns improved in three cases, while no significant changes were seen in two. Anesthetics were weaned in five of six patients within 72-hours post-ECT. No adverse events were associated with ECT.

\section{Conclusions}

As a salvage therapy in SRSE, ECT stopped seizures in 6 patients with ongoing SRSE and improved electrographic patterns in 6 of 8 patients. Electroconvulsive therapy was associated with reduction of anesthetic burden and did not cause any adverse events. Our results suggest that ECT may be safe and possibly efficacious for SRSE. 


\section{Poster 271}

\section{Session 1 or 2}

Michael Miksa, Rishi Malhotra, Alexis Boro

Montefiore Medical Center - Pediatric Critical Care, New York, NY, United States

\section{Background \& Purpose}

Status epilepticus is present in 4-7\% of pediatric ICU admissions. Initiating EEG studies in a timely manner is challenging, particularly on off-hours, causing delays in appropriate management. We assessed the impact of using Ceribell ${ }^{\circledR}$ rapid response EEG on time to diagnosis in this population.

\section{Methods}

We compared time to initial EEG reading between conventional (cEEG) and rapid response EEG (RR-EEG) in patients admitted to a pediatric ICU in whom status epilepticus was suspected over one year. Based on study availability and physician preference, either study was initiated and specialist reading was obtained. Times to EEG start and reading, findings, clinical management and functional outcomes data were collected. Chi-squared or Wilcoxon Rank Sum tests were used where appropriate.

\section{Results}

Complete data was available for 42 patients. The median RR-EEG deployment time was 19 min (IQR: 10$78, \mathrm{n}=17)$ compared to $230 \mathrm{~min}($ IQR: $123-356, \mathrm{n}=25)$ using cEEG $(\mathrm{p}<0.001)$. EEG reports were available within $46 \mathrm{~min}$ (IQR: 29-83) vs. $141 \mathrm{~min}$ (IQR: 60-446, $\mathrm{p}=0.005)$ from the start of the study. The probability of detecting seizures was $18 \%$ and $24 \%$, respectively $(p=0.9128)$. Changes in management of seizures included de-escalation of therapy (36\%), escalation (33\%) and maintenance of current medications (31\%), without any significant differences between the groups. The groups differed in age (11 years [IQR: 8-17] vs. 4 years [3-10], $p=0.0237$ ), neurological diagnosis (acute encephalopathy (41\%) vs. epilepsy (56\%), $p=0.0421$ ) and intubation status (76\% vs. $36 \%, p=0.0236)$, respectively. Outcomes were not different between both groups.

\section{Conclusions}

RR-EEG enabled more rapid initiation and reporting of the initial EEG findings, with the two modalities detecting seizures in a similar proportion of cases. One limitation of the technology in the pediatric setting is the minimum head circumference requirement $(48 \mathrm{~cm})$ for the head band. 
Poster 272

\section{Statin Treatment and Early Seizures Among Acute Ischemic Stroke Patients Undergoing EEG Monitoring}

Jiping Zhou, Paula Sanches, M. Brandon Westover, Eric S. Rosenthal, Lidia Moura, Sahar F. Zafar

Department of Neurology, Wayne State University School of Medicine/Detroit Medical Center, Detroit, MI, United States

\section{Background \& Purpose}

Early clinical and electrographic seizures can occur in up to $17 \%$ risk of acute ischemic stroke (AIS) patients and are associated with worse outcomes. Statin use in the acute phase has been reported to be associated with a lower incidence of post-stroke early clinical seizures through its underlying neuroprotective effect. In this study, we sought to examine whether pre-stroke statin use and initiation or escalation of statin treatment during the acute phase lower the risk of post-stroke early clinical and electrographic seizures.

\section{Methods}

We performed a retrospective cohort study of AIS patients admitted to a single center between 20122019. All subjects underwent $>18$ hours of continuous electroencephalography monitoring (cEEG) within 7 days of stroke. Exposures were 1) pre-stroke statin treatment, and 2) Initiation or escalation of statin treatment within 72 hours of stroke. The primary outcome was the development of early clinical and/or electrographic seizures (ES) within 7 days of stroke onset. Logistic regression analyses were used to assess the relationship between statin use and post-stroke ES.

\section{Results}

Of the 146 enrolled subjects, 67 (45.9\%) received pre-stroke statin treatment. $122(88.1 \%)$ subjects had an initiation or increased intensity of statin treatment during the acute phase. $33(22.6 \%)$ subjects had ES. After adjustment for baseline clinical covariates and illness severity, pre-stroke statin therapy was associated with lower odds for acute ES (odds ratio [OR] 0.67, 95\% $\mathrm{Cl} 0.25-1.82, \mathrm{p}=0.431$ ), however, these findings did not reach statistical significance. Initiation or escalation of statin treatment intensity in the post-stroke acute phase was associated with a lower risk of ES (OR 0.29, $95 \mathrm{Cl} \% 0.09-0.89$, $\mathrm{p}=0.031$ ).

\section{Conclusions}

Increased intensity of statin treatment in the acute phase may be associated with a lower risk of both clinical and electrographic seizures in the early post-stroke phase. Larger prospective studies are indicated to test this hypothesis. 
Poster 273

\section{Successful Detection of Infraslow Activity Including Direct Current Shift using Scalp Electroencephalogram with a Time Constant of 2 Seconds for Anoxic Brain Injury after Cardiac Arrest}

\section{Satoshi Egawa}

TMG Asaka Medical Center, Neuro ICU, Department of Neurosurgery, and Stroke and Epilepsy Center, Saitama, Japan

\section{Background \& Purpose}

A previous study has shown the efficacy of scalp electroencephalogram (s-EEG) with a time constant of 2 $s$ in detecting infraslow activity (ISA) including direct current (DC) shifts. However, its feasibility in the field of neurocritical care has not been assessed before. Here, to describe the feasibility of using s-EEG to detect ISA, we report cases in which ISA was successfully detected for anoxic brain injury after cardiac arrest (CA).

\section{Methods}

Four patients with ISA participated in this case series. Those participants received continuous EEG monitoring (EEGM) in the intensive care unit. The data were recorded using scalp EEG with a frequency filter of $0.08 \mathrm{~Hz}$ (time constant of $2 \mathrm{~s}$ ) using the international 10-20 system were analyzed.

\section{Results}

Four patients demonstrated some ISAs. All patients demonstrated convulsive seizures after resuscitation. Then, the patients received targeted temperature management with neuromuscular blocking agents and sedations under EEGM. Of those, one patient was resuscitated from CA caused by heart failure, two CAs were caused by hangings, and one by subarachnoid hemorrhage (SAH). The findings of EEGM showed seizure and passive DC shifts with high-frequency oscillations for three patients. One patient with SAH showed positive slow discharge continuing for $3 \mathrm{~s}$, superimposed with brief potentially ictal rhythmic discharges.

\section{Conclusions}

In conclusion, some ISAs could be detected using s-EEG with a time constant of $2 \mathrm{~s}$ for anoxic brain injury after CA with neuromuscular blocking agents. Most of ISAs were DC shifts. 
Poster 274

The Use of Quantitative EEG Spectral Analysis to Characterize IV Ganaxolone PK/PD characteristics in Patients with Refractory Status Epilepticus

\author{
Henrikas Vaitkevicius, Eva Rybak, Maxim Meersman, Pieter van Mierlo, Maciej Gasior \\ Marinus Pharmaceuticals, Radnor, PA, United States
}

\title{
Background \& Purpose
}

Ganaxolone is a synthetic neuroactive steroid that acts as a positive allosteric modulator of GABAA receptors and is in clinical development for the treatment of status epilepticus (SE). Ganaxolone has a large volume of distribution and complex pharmacokinetic properties and produces rapid and profound background EEG changes. This study evaluated CNS-specific pharmacokinetic and pharmacodynamic properties of intravenous (IV) ganaxolone using spectral analysis of EEG during a Phase 2 study in refractory SE (RSE) (NCT03350035).

\section{Methods}

Continuous EEG data was evaluated from initial 5 patients with RSE enrolled in the phase 2 study. The alpha/delta ratio and suppression ratio were computed from 30 min before through 48 hours after initiation of IV ganaxolone. The percentage change relative to the $30 \mathrm{~min}$ baseline period was computed. Alpha/delta power and suppression ratio were correlated with observed and predicted serum ganaxolone levels.

\section{Results}

The alpha/delta ratio showed a remarkable increase immediately after the start of IV ganaxolone in $5 / 5$ patients. The median value of time to peak and corresponding percentage increase were $5 \mathrm{~min}$ and $87 \%$, respectively, which matched the observed median time to SE cessation. For the suppression ratio, an increase was observed in 4/5 patients, with a median time to peak of 6:30 min and percentage increase of $64 \%$. A significant positive linear correlation was found between the suppression ratio and serum ganaxolone levels with $r$-squared greater than 0.87 in 4 out of 5 patients.

\section{Conclusions}

This preliminary analysis suggests that PK/PD qualities of IV ganaxolone could be successfully characterized by quantitative EEG measures which closely correlate with clinical observations. 


\section{Poster 275}

\section{Association of Valproic Acid Use and Discharge Outcomes After Aneurysmal Subarachnoid Hemorrhage}

Matthew Cobler-Lichter, Ali Daneshmand, Hayley Tatro, Ava Cascone, Joanna Yang, David Greer, Anna Cervantes-Arslanian, Courtney Takahashi, Charlene Ong, Mohamad K Abdalkader,Hormuzdiyar H. Dasenbrock, Thanh Nguyen, David Chung, Kushak Suchdev,

Boston Medical Center Department of Neurology, Boston, MA, United States

\section{Background \& Purpose}

Cortical spreading depolarization (SD) is implicated in the pathogenesis of delayed cerebral ischemia (DCI) in subarachnoid hemorrhage (SAH). Valproic acid (VPA) is effective in aborting SD-mediated secondary injury following SAH in rodents, but the effect of VPA on SAH outcomes in humans is unknown.

\section{Methods}

We conducted a retrospective analysis of a prospectively collected 123 adult patients with nontraumatic SAH referred to Boston Medical Center from 2014 to 2020. Eighty-seven were aneurysmal and 36 were angiogram-negative. The outcomes analyzed for this study were radiographic vasospasm, $\mathrm{DCl}$, and discharge modified Rankin Scale (mRS). Stepwise logistic regression analyses were conducted to determine the association between VPA use and outcome variables. We used a final logistic regression model that included age, sex, World Federation of Neurological Surgeons (WFNS) score and VPA use.

\section{Results}

Fifteen percent of patients (18/123) were given VPA. Adjusted analyses showed that VPA use did not have a significant association with $\mathrm{DCl}(\mathrm{OR}=0.87,95 \% \mathrm{Cl}$ : $0.14-5.41)$ or vasospasm $(\mathrm{OR}=0.68,95 \% \mathrm{Cl}$ : 0.22 - 2.13). Adjusted logistic regression analysis did not show a statistically significant association between VPA use and discharge $\mathrm{mRS}>3(\mathrm{OR}=0.52,95 \% \mathrm{Cl}: 0.12-2.24)$. Increased WFNS (OR $=0.46$, $95 \% \mathrm{Cl}: 0.36-0.59)$ and increased/older age $(\mathrm{OR}=0.96,95 \% \mathrm{Cl}: 0.94-0.98)$ were associated with worse discharge $\mathrm{mRS}$. Decreased/younger age $(\mathrm{OR}=0.96,95 \% \mathrm{Cl}: 0.93-0.99)$ and increased WFNS $(\mathrm{OR}=1.42$, $95 \% \mathrm{Cl}: 1.11-1.83)$ were predictive of radiographic vasospasm but not $\mathrm{DCl}$. There were no complications associated with VPA administration.

\section{Conclusions}

We did not observe any effect of VPA administration on the rate of vasospasm, $\mathrm{DCl}$, and discharge $\mathrm{mRS}$ in SAH patients. Future research regarding CSD and VPA using a prospective, adequately powered, multicenter approach with intracranial monitoring is needed. 


\section{Poster 276}

\section{Comparison of Waveforms between Noninvasive and Invasive ICP Monitoring}

Fabiano Moulin de Moraes, Gisele Sampaio Silva

Federal University of São Paulo - Department of Neurology and Neurosurgery, São Paulo, Brazil

\section{Background \& Purpose}

Due to the limitations of the gold standard model for measuring intracranial pressure (ICP) (placement of an intra-cerebral catheter), the use of noninvasive ICP (nICP) wave morphology have been proposed to add clinically relevant information. This study aimed to compare the wave morphology between the gold standard and a new nICP monitor.

\section{Methods}

We prospectively collected data on adults admitted to a tertiary center neurocritical care unit with subarachnoid hemorrhage (SAH), intracerebral hemorrhage (ICH), and ischemic stroke (IS) in whom invasive ICP monitoring had been placed. Simultaneously, two ICP wave parameters (time-to-peak [TTP] and P2/P1 ratio) were analyzed. Intracranial hypertension was defined as an invasively measured ICP > $22 \mathrm{mmHg}$.

\section{Results}

We studied 18 patients on 60 occasions, $55 \%$ females, mean age 52 years +/- SD (SAH =14; ICH = 3; IS=1). A total of 197.400 waves (2495 minutes) both from the invasive ICP and the nICP monitor were sliced into $1 \mathrm{~min}$-length segments, and from the mean pulse, we determined the Time to Peak (TTP) and $\mathrm{P} 2 / \mathrm{P} 1$ ratio. The median invasively measured ICP was 13 [9.8-16.2] $\mathrm{mmHg}$, and intracranial hypertension was present in 18 occasions (30\%). The agreement between the invasive and noninvasive methods for wave morphology was strong for the P2/P1 ratio and moderate for TTP using categoric (kappa agreement 88,1 and $71,3 \%$, respectively) and continuous (Intra-class correlation coefficient [ICC] 0,831 and 0,584 , respectively) measures.

\section{Conclusions}

The new nICP wave morphology monitor showed good agreement with the standard invasive method and creates the opportunity to widen the use of the ICP wave morphology in clinical practice. 


\section{Poster 277}

\section{Cortical Spreading Depolarizations in the Prechiasmatic Injection Model of Subarachnoid Hemorrhage}

James H. Lai, Tao Qin, Sava Sakadžić, Cenk Ayata, David Y. Chung

Massachusetts General Hospital Neurology, Boston, MA, United States

\section{Background \& Purpose}

Cortical spreading depolarizations (CSD) are associated with worse outcomes in patients with aneurysmal subarachnoid hemorrhage (SAH). Animal models are required to assess whether CSDs can worsen outcomes or are an epiphenomenon; however, little is known about the presence of CSDs in existing animal models. Therefore, the current study was designed to determine if CSDs occur in a mouse model of SAH during the acute peri-induction period.

\section{Methods}

A total of 36 mice were included in the study. The anterior prechiasmatic injection model was utilized for induction of SAH or control saline injection under isoflurane anesthesia. The injection needle was inserted through the mouse's olfactory bulb with the needlepoint terminating at the base of the skull. Changes in cerebral blood volume were assessed with optical intrinsic signal imaging using a green LED with a wavelength at the isosbestic point of oxy- and deoxyhemoglobin $(530 \mathrm{~nm})$.

\section{Results}

CSDs occurred in $100 \%$ of mice in the ipsilateral hemisphere following needle insertion (CSD1). Salineinjected mice had $100 \%$ survival $(n=10)$. Blood-injected mice had an $88 \%$ survival $(n=23 / 26)$. A second, delayed, CSD ipsilateral to CSD1 occurred in 31\% of blood-injected mice. An increase in the time interval between CSD1 and blood injection was associated with the occurrence of CSD2 in blood-injected mice ( 26.4 vs $72.7 \mathrm{sec}, \mathrm{p}<0.0001, \mathrm{n}=18$ and 8 ). We observed one blood-injected animal with a second CSD in the contralateral hemisphere and observed terminal CSDs in mice that died following SAH injection.

\section{Conclusions}

The prechiasmatic injection model of SAH includes CSDs that occur at the time of needle insertion. The occurrence of subsequent CSDs depends on the timing between CSD1 and blood injection. Further work is needed to determine the effect of multiple early CSDs on outcomes following SAH. 


\section{Poster 278}

\section{Dexamethasone Use for Headache Pain after Subarachnoid Hemorrhage and Proinflammatory Cells in Cerebral Spinal Fluid}

EK Glisic, SV Ryzhov, JT deKay, C. Lucy, V. Carpenter, T. May, R. Riker, P. Lerwick, DB Seder

Maine Medical Center, Department of Pharmacy, Portland, ME, United States

\section{Background \& Purpose}

We hypothesized that dexamethasone (DEX) use for headache (HA) after subarachnoid hemorrhage (SAH) suppresses the activation of proinflammatory cells in cerebral spinal fluid (CSF) and decreases HA pain.

\section{Methods}

IRB-approved, prospective, observational study of non-traumatic SAH among patients requiring ventriculostomy. Inpatient pain scores using Numeric Rating Scale (NRS, 0-10) were recorded over postbleed days (PBD) 0-10. Blood and CSF were collected on PBD 1, 3, 5, 7, and 10. Flow cytometric analysis was used to determine markers of proinflammatory cell activation.

\section{Results}

Among 16 patients, 81\% female, mean age $59.9 \pm 12.3$ years with $\mathrm{HH}$ grade I (1/16), II (4/16), III (4/16), IV $(6 / 16)$ and $V(1 / 16)$, eight patients received DEX. DEX patients were $88 \%$ female, age $61.3( \pm 11.8)$ years and median $\mathrm{HH}$ grade 2 , compared to $75 \%$ female, age $58.5( \pm 13.4)$ years and median $\mathrm{HH}$ grade 4 among NO-DEX patients. The mean of max NRS on day 0 for DEX group vs NO-DEX group was $8.8( \pm 1.8)$ vs $4( \pm 0)$, respectively. The mean of max NRS on day 7 for DEX group vs NO-DEX group was $4.7( \pm 2.5)$ vs $4.7( \pm 4.2)$, respectively. Unsupervised clustering analysis revealed a decrease in the expression of CD14, a coreceptor of toll-like receptors, on neutrophils (mean fluorescence intensity (MFI): 183 vs. 266, DEX vs. NO-DEX, $p=0.049$, unpaired t test) and monocytes (MFI: 910 vs. 1105, DEX vs. NO-DEX, $p=0.047$, unpaired t test) from the blood of DEX group. A decrease was found in number of neutrophils in CSF obtained from patients in DEX group (2.6 vs. 6.1, DEX vs. NO-DEX, $p=0.045$, unpaired t test).

\section{Conclusions}

Dexamethasone treatment was associated with suppression of myeloid cell activation after nontraumatic SAH. Patients receiving dexamethasone had higher initial pain scores and lower Hunt and Hess grades, but similar subsequent pain scores. Further studies are warranted to determine antiinflammatory mechanisms involved in steroid-dependent control of headache after SAH. 


\title{
Poster 279
}

\section{International Nutritional Survey of Nutrition Practices and Nutrition Intake on Outcome after Subarachnoid Hemorrhage}

\author{
Neeraj Badjatia, Alice Ryan, H. Alex Choi, , Gunjan Y. Parikh, , Xuran Jiang, Andrew G. Day, Daren K. \\ Heyland
}

Program in Trauma, Department of Neurology, University of Maryland School of Medicine, Baltimore, $\mathrm{MD}$, United States

\section{Background \& Purpose}

Single center studies have reported an association between low caloric intake(Cl), protein intake(PI), and poor outcome after subarachnoid hemorrhage(SAH). We investigated whether outcomes vary by $\mathrm{PI}$ or $\mathrm{Cl}$ in $\mathrm{SAH}$ patients enrolled in an international survey of critical care nutrition practices, adjusting for the nutritional risk as judged by the modified NUTrition Risk in the Critically III (mNUTRIC) score.

\section{Methods}

Data from SAH patients enrolled in the International Nutrition Survey(INS) was utilized to describe the characteristics, outcomes and nutrition practices. A subgroup of consecutive patients from when mNUTRIC score was captured examined the association between $\mathrm{Cl}$ and $\mathrm{PI}$ and time to discharge alive(TTDA) from hospital using Cox regression models, adjusting for nutrition risk classified by the mNUTRIC score as low(0-4) or high(5-9).

\section{Results}

There were 489 SAH patients( $57 \%$ female with a mean \pm SD age $57.5 \pm 13.9$ years, BMI of $25.9 \pm 5.3 \mathrm{~kg} / \mathrm{m} 2$ and APACHE-2 score 19.4 \pm 7.0 ) enrolled from 201 ICUs worldwide. Majority of patients(85\%) received enteral nutrition(EN) only, with a time to initiation of EN of $35.4 \pm 35.2$ hours. Targeting a hourly goal rate was the most common approach (80\%) with 64\% having EN interrupted for radiology, airway or surgical procedures. Patients received a $\mathrm{Cl}$ of $14.6 \pm 7.1$ calories $/ \mathrm{kg} / \mathrm{day}$ and $\mathrm{PI} 0.7 \pm 0.3 \mathrm{grams} / \mathrm{kg} / \mathrm{day}$ corresponding to $59 \%$ and $55 \%$ of total prescribed $\mathrm{Cl}$ and $\mathrm{PI}$ respectively. The mNUTRIC score was $3.4 \pm 1.8$ in the subgroup population $(n=226)$. Increased $\mathrm{Cl}$ and PI were associated with faster TTDA among high mNUTRIC patients(HR per $20 \%$ of prescription received $=1.34[95 \% \mathrm{Cl}, 1.03-1.76]$ for $\mathrm{Cl}$ and 1.44[1.07-1.93] for PI), but not low mNUTRIC patients(CI: HR=0.95[0.77-1.16] PI:0.95[0.78-1.16]).

\section{Conclusions}

Results from the INS confirm previous single center study findings that SAH patients are underfed and achieving greater $\mathrm{Cl}$ and $\mathrm{PI}$ in hi risk SAH patients is beneficial. Furthermore, we identify the mNUTRIC as a useful tool to identify SAH patients that benefit most from artificial nutrition. 
Poster 280

\title{
Machine Learning Analysis of Proteomic Data Reveal New Biomarkers and for Delayed Cerebral Ischemia
}

\author{
H. Alex Choi, Jude PJ Savarraj, Aaron M. Gusdon, Atzhiry S. Paz, Glenda L. Torres, Xuefang Ren \\ UT Health Neuroscience, Houston, TX, United States
}

\section{Background \& Purpose}

Delayed cerebral ischemia (DCl) is the most serious secondary complication after subarachnoid hemorrhage $(\mathrm{SAH})$. $\mathrm{DCl}$ is characterized by cerebral infarction and/or neurological worsening that affects $\sim 30 \%$ of SAH patients between 4-21 days after SAH. Proteomic biomarkers and machine learning (ML) based models can be used to predict DCl.

\section{Methods}

We undertook targeted and untargeted interrogation of proteins using mass spectrometry and 'proximity extension assay' (PEA) technology in plasma samples from $80 \mathrm{SAH}$ subjects ( 50 no-DCl and 30 $\mathrm{DCl}$, matched for age, sex and modified Fisher score (mFS)) collected at $<48$ hours after admission. We analyzed the prognostic ability of individual proteins and a panel of proteins using statistical and machine learning methods.

\section{Results}

Interferon gamma-induced protein-10, Guanylate-binding protein-2, Neurexophilin-1, Scavenger receptor class A member-5, and GDF8 were significantly lower in the $\mathrm{DCl}$ group compared to no-DCl group ( $p<0.01$, student's t-test) after adjusting for multiple comparisons. LRG-1 was significantly higher in those who proceeded to develop $\mathrm{DCl}$. Including all six proteins in $\mathrm{ML}$ models resulted in the highest area under the curve (AUC) for prediction of $D C I$. While individual proteins had reasonable prognostic value (AUC ranging from 0.62 to 0.69 , with each prognosticated $\mathrm{DCI}$ had better than the $\mathrm{mFS}$ ), a combination of proteins resulted in the highest AUC $(0.8)$, which was significantly $(p<0.05$, DeLong Test) higher than the $\mathrm{mFS}$ score alone (AUC = 0.58).

\section{Conclusions}

The proposed biomarkers are novel in SAH research and have strong biological relevance to the pathophysiology of $\mathrm{DCl}$. As $\mathrm{DCl}$ is a complex multifactorial disease process, a combination of molecules, which represent different facets of the disease process, are needed for improved prediction. ML models are useful in leveraging the combined prognostic capability of these proteins. Besides serving as prognostic biomarkers, these proteins could be important therapeutic targets for future studies. 
Poster 281

\section{Methamphetamine is Associated with Younger Age at Rupture in Aneurysmal Subarachnoid Hemorrhage}

Caitlin Richardson-Royer, Nathan Beird, Holly Hinson

Oregon Health \& Science University, Department of Neurology, Portland, OR ,United States

\section{Background \& Purpose}

Methamphetamine users make up approximately $0.6 \%$ of the general population over age 12 , but up to $17 \%$ of aneurysmal subarachnoid hemorrhage patients (aSAH). While methamphetamine users appear to have worse outcomes than age-matched controls, the mechanism(s) of this association is not fully understood. We hypothesized that methamphetamine use may influence aneurysm characteristics such as size at rupture.

\section{Methods}

Consecutive aSAH patients from October 2018 to August 2020 were identified from a custom-designed electronic health record query based on problem lists and populated into a database. Demographics, hospital course, aneurysm characteristics were obtained from this database, while largest aneurysm dimension measurements were abstracted from angiography reports. Data is presented as counts, percentages, means $( \pm S D)$ or medians $[I Q R]$ as appropriate, with significance set at $p \leq 0.05$.

\section{Results}

Ninety-eight patients were identified by the query, of whom 59 had aneurysms detected on CT or angiography. Overall, $12.25 \%$ (12/98) of SAH patients and $16.95 \%$ of the aneurysmal SAH patients were identified as having a history of methamphetamine use or an admission toxicology test positive for amphetamines. Those with meth use history were younger than non-users ( $48 \pm 14 \mathrm{v} .60 \pm 13$ years old, $\mathrm{P}=0.02)$. There were no differences in sex $(50 \%$ v. $58 \%$ female, $p=0.25)$, median Hunt and Hess (3 [2-5] v. 2 [2-4], $p=0.07$ ), or median modified Fisher (4 [3-4] v. 4 [2-4], $p=0.12$ ) scales between meth users and non-users. Mean largest-diameter size of the ruptured aneurysms were $8.6 \pm 5 \mathrm{~mm}$ in meth users, and $6.5 \pm 4 \mathrm{~mm}$ in non-users $(P=0.24)$. Culprit aneurysms were identified by angiography in a similar number of cases $(83 \%$ v. $63 \%, P=0.21)$ in meth users versus non-users.

\section{Conclusions}

We found that patients with current or historical methamphetamine use suffered aneurysm rupture at younger ages. There may be an association between rate of aneurysm growth and methamphetamine use, but larger studies are required to assess this relationship. 


\section{Poster 282}

\section{Monocyte Mitochondrial Respiration is Impaired after Aneurysmal Subarachnoid Hemorrhage}

Aaron M, Gusdon, Hua Chen, Jude Savarraj, Atzhiry Paz, Glenda L. Torres, Pramod K. Dash, H. Alex Choi

University of Texas Health Science Center, Department of Neurosurgery, Houston, TX, United States

\section{Background \& Purpose}

Aneurysmal subarachnoid hemorrhage (aSAH) causes a systemic inflammatory response, which has been linked to worse outcomes. Increased activity of the innate immune system with mobilization of proinflammatory monocytes occurs peripherally driving cytokine production, and these monocytes also traffic to the brain. Metabolic changes precede activation of inflammatory cells, with a shift from oxidative to glycolytic metabolism driving proinflammatory activation. We hypothesized that early metabolic shifts in peripheral monocytes would occur after aSAH and that this could be prevent by use of alternative substrates.

\section{Methods}

Subjects with aSAH ( $N=8)$ were enrolled within 24 hours of onset along with age matched controls $(\mathrm{N}=4)$. Monocytes were isolated from whole blood immediately after collection by negative selection using an EasySep Isolation Kit (Stemcell Technologies). In situ mitochondrial respiration was assessed using a Seahorse extracellular flux analyzer. Monocytes were plated at 150,000 cells per well in at least triplicate with cells from control and SAH patients on each plate. Basal respiration was assessed initially, followed by the additional of oligomycin to assess proton leak, FCCP to assess maximal respiratory capacity, and rotenone/antimycin to assess non-mitochondrial respiration.

\section{Results}

Basal mitochondrial respiration (31.2+/-4.12 vs $63.1+/-8.41 \mathrm{pmol} / \mathrm{min}$ ) and FCCP-induced maximal respiratory rates (34.9+/-9.23 vs $99.4+/-15.04 \mathrm{pmol} / \mathrm{min}$ ) were significantly lower in aSAH subjects compared with controls. Oxygen consumption driving proton leak (24.1+/-2.11 vs $25.7+/-9.82)$ and nonmitochondrial oxygen consumption (21.6+/-1.89 vs 19.4+/-4.46) were not significantly different comparing the two groups. Treatment of monocytes from aSAH subjects with alpha-ketoglutarate or palmitate increased basal and maximal respiratory rates.

\section{Conclusions}

Monocyte mitochondrial function is impaired early after aSAH. Monocyte mitochondrial respiration can be bolstered by treatment with substrates promoting oxidative metabolism. Alternative substrates may represent a treatment strategy to improve systemic metabolism after aSAH and attenuate systemic innate immune activation and subsequent inflammation. 
Poster 283

\section{The Influence of Blood Volume Analysis on the Clinical Management of Non-Traumatic Subarachnoid Hemorrhage}

Lindsey Carvalho, John Holton-Burke, Nathan Beird, Kelly Batte, Justin Cetas, H.E. Hinson

Oregon Health and Science University, neurology, Portland, OR, United States

\section{Background \& Purpose}

Non-traumatic subarachnoid hemorrhage (SAH) can cause complicated irreversible neurological injury. A key variable in the management of SAH is intravascular volume status. Blood volume analysis (BVA) is a non-invasive method of quantifying intravascular volume status using radioisotope tagged-albumin. BVA has been validated for use in surgical ICUs, but its utility for clinical decision making in SAH is unclear. We hypothesized that intravascular volume assessments would influence clinical decision making in SAH.

\section{Methods}

Four clinical scenarios, consisting of hypothetical patients with SAH were presented in an anonymous IRB-exempt multiple-choice survey of 10 questions to Neurocritical care providers at a single institution neuroscience ICU. Nineteen attending physicians and advanced practice practitioners were eligible to participate. The scenarios described a variety of clinical problems including hyponatremia, vasospasm, and anemia. Participants were asked for their next step in management of volume status, with answers being volume restriction, hyperosmolar fluid, blood transfusion, IVF bolus, diuresis, and none of the above. Subsequently, BVA data was then provided, and participants were asked how they would manage the patient with this additional data. Qualitative differences between pre/post BVA management were analyzed.

\section{Results}

Nine (47\%) participants responded to the survey. Of the 36 answers, $69 \%(n=25)$ responded with different management strategies after being provided with BVA data, while $28 \%(n=10)$ responses did not change in light of the data and 1 question was left blank. All participants indicated a preference for BVA over invasive hemodynamic monitoring if given an option of either modality to monitor volume status in SAH due to the former's less invasive nature.

\section{Conclusions}

In this single institution study, BVA results appeared to influence clinical management in hypothetical scenarios in $\mathrm{SAH}$, though there was notable inter-provider heterogeneity in the management strategies chosen. Future work will focus on the study of a protocol informed by BVA data. 


\title{
Poster 284
}

\section{The ROLE of HYPERBARIC OXYGEN THERAPY in Subarachnoid HEMORRHAGE and CEREBRAL ISCHEMIA}

\author{
Ali A. Alsarah, Alycia N. Montgomery, Talia J. Passarelli, Sajid S. Suryia, Lance M, Larson, Diana Greene- \\ Chandos, Michel Torbey, Omar M. Hussein
}

University of New Mexico Hospital - Department of Neurology, Albuquerque, NM, United States

\section{Background \& Purpose}

Subarachnoid hemorrhage is a neurologic emergency associated with significant morbidity and mortality. Several neuroinflammatory and ischemic changes have been demonstrated to contribute to the adverse outcome. The resultant severity of cerebral ischemia secondary to subarachnoid hemorrhage (SAH) has been one of the determining factors to signify the outcome. Using hyperbaric oxygen therapy (HBOT) in the early stages following the ictus has been demonstrated to be a beneficial treatment modality in several human and animal study models. Through delivering a fraction of superatmospheric pressure and securing high oxygen tension in the brain tissue in an attempt to reverse the pathological changes secondary to the SAH and its associated ischemic changes, it has shown that it may carry a favorable outcome.

\section{Methods}

A total of 52482 articles were found using the initial terms. Exclusions were made for the following reasons: if the article was a case report or a review article, if it was a partial article only, if the article was repeated, if the article was not peer-reviewed and if there was a lack of access to an article. There were no language restrictions on the search, and some relevant articles did require translation in order to be included in this review, and this was done in an attempt to eliminate bias. There was not a restricted time frame on the articles due to there being limited primary research regarding HBOT and SAH.

\section{Results}

HBOT related neuroprotection has been attributed to different mechanisms that happen at different stages in the early ischemic changes' major mechanisms:1)Oxidative stress reduction,2)Reducing the inflammation,3) Improve the cerebral metabolic rate,4) Prevention of Apoptosis

\section{Conclusions}

We are presenting a systematic review for animal and human studies to explain the accompanying changes following different modalities of delivering HBOT to have an explanation about what dose, timing, and frequency can contribute to a better outcome in patients with subarachnoid hemorrhage to prevent worsening of the cerebral ischemia.HBOT pressures between 2.4 to 3.0 ATA have been shown to be effective while avoiding any oxygen toxicity or seizures and are associated with improved longterm outcomes based on findings on human model studies. Further future studies are required to focus on the timing of initiation of the treatment following the insult of SAH. 